\title{
High Speed and Wide Bandwidth Delta-Sigma ADCs
}

M. Bolatkale 


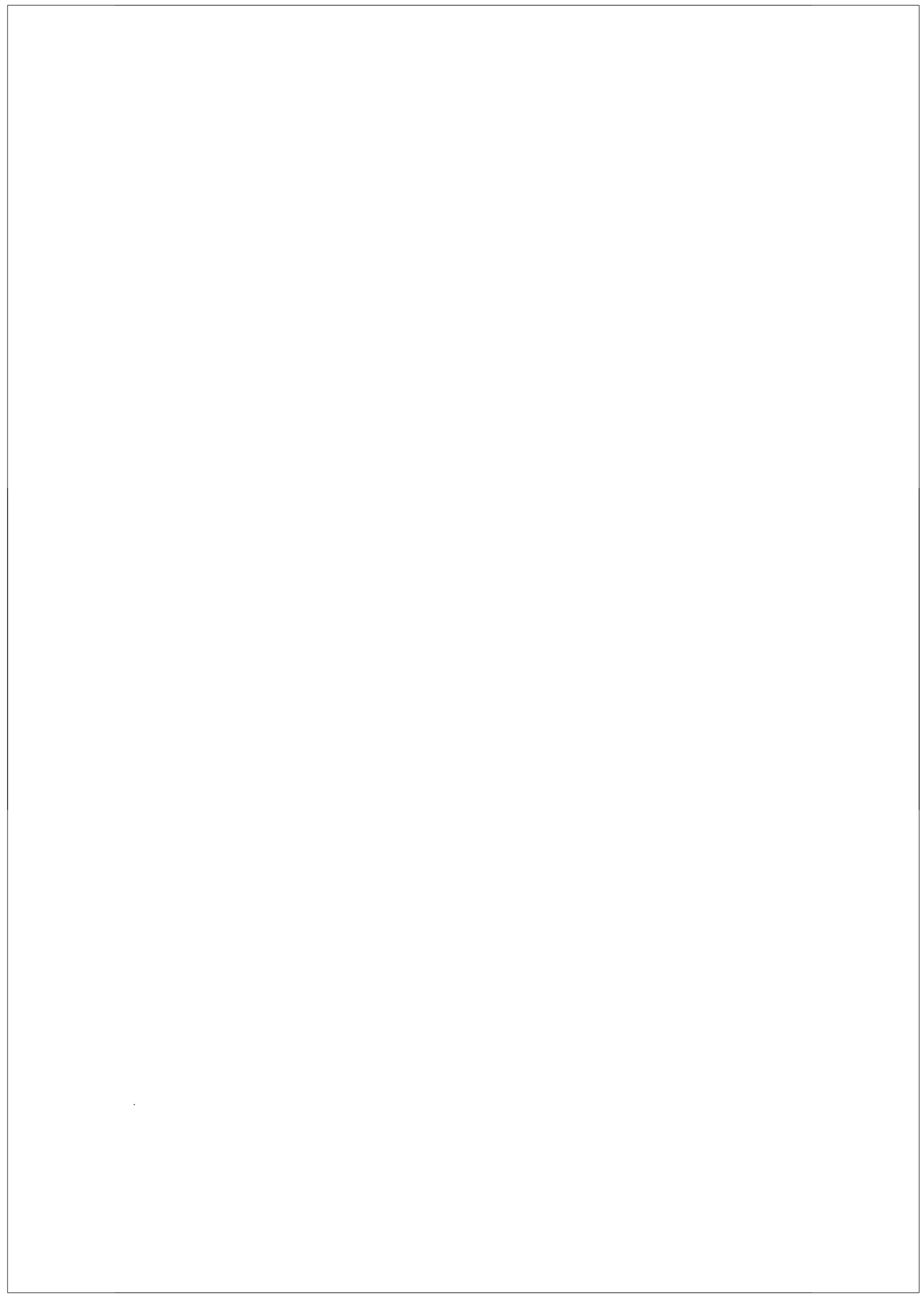




\title{
High Speed and Wide Bandwidth Delta-Sigma ADCs
}

\author{
Proefschrift \\ ter verkrijging van de graad van doctor \\ aan de Technische Universiteit Delft, \\ op gezag van de Rector Magnificus prof. ir. K.C.A.M. Luyben, \\ voorzitter van het College voor Promoties, \\ in het openbaar te verdedigen \\ op dinsdag 22 october 2013 om 12:30 uur
}

door

Muhammed BOLATKALE,

Master's Degree in Electronic Engineering Delft University of Technology, The Netherlands

geboren te Kahramanmaraş, Turkey. 
Dit proefschrift is goedgekeurd door de promotoren:

Prof. dr. ir K.A.A. Makinwa

Prof. dr. ir. L.J. Breems

Samenstelling promotiecommissie:

\section{Rector Magnificus}

Prof. dr. ir. K.A.A. Makinwa

Prof. dr. ir. L.J. Breems

Prof. dr. ir. Ing. M. Ortmanns

Prof. dr. ir. R.B. Staszewski

Prof. dr. ir. B. Nauta

Dr. ir. M.A.P. Pertijs

Dr. ir. M. Pelgrom

Prof. dr. ir. E. Charbon

\section{voorzitter}

Technische Universiteit Delft, promotor

Technische Universiteit Eindhoven, promotor

Ulm University, Germany

Technische Universiteit Delft

Universiteit Twente

Technische Universiteit Delft

Pelgrom Consulting

Technische Universiteit Delft, reservelid

The research described in this thesis was funded by NXP Semiconductors B.V.

Published and distributed by: Ipskamp Drukkers B.V.

ISBN 978-94-6191-877-2

Copyright (c) 2013 by M. Bolatkale

All rights reserved. No part of the material protected by this copyright notice may be reproduced or utilized in any form or by any means, electronic or mechanical, including photocopying, recording or by any information storage and retrieval system, without written permission of the author.

Printed in The Netherlands 
Aileme ve bana destek olan tüm herkese To my parents and to the people who have supported me 


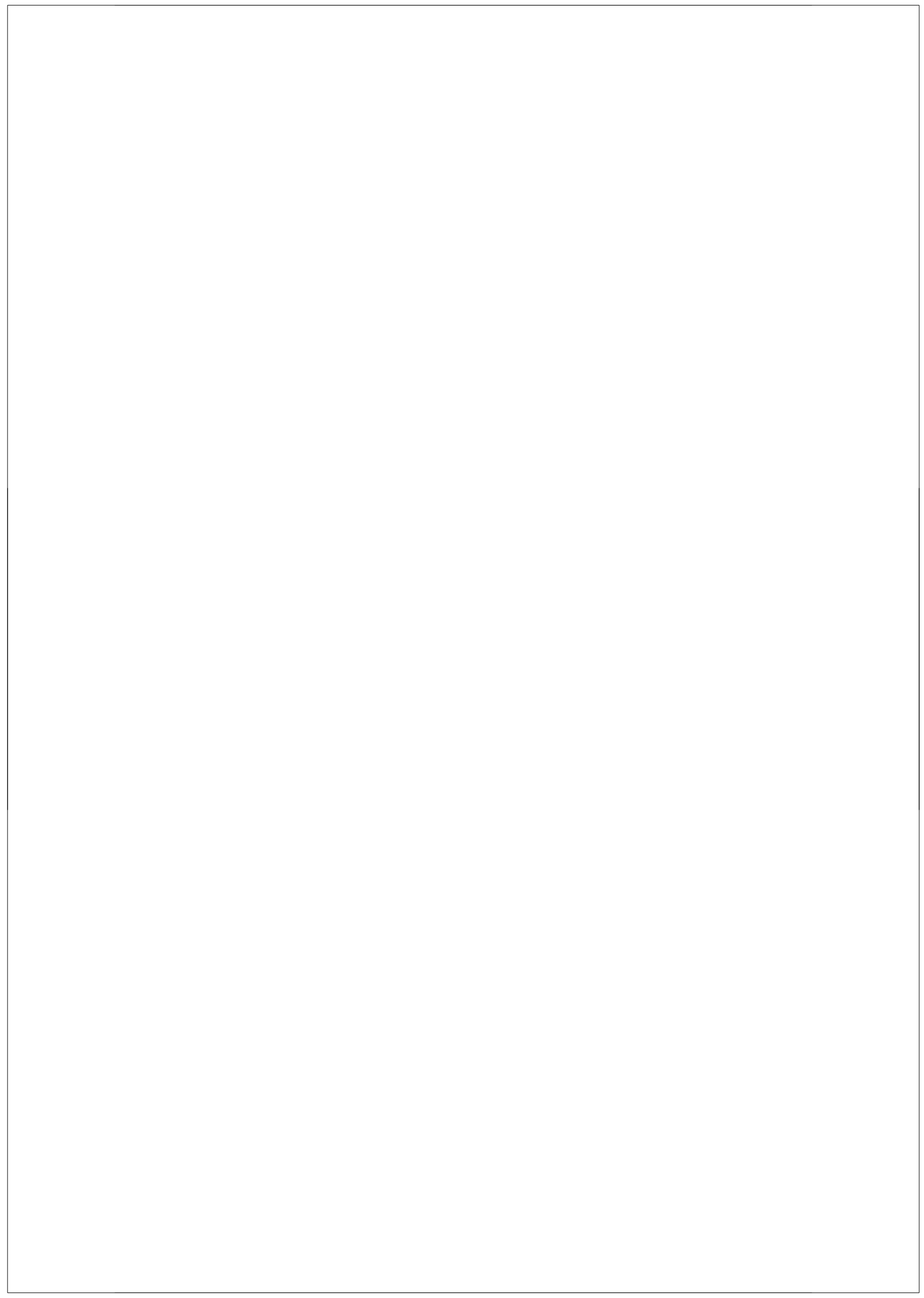




\section{Contents}

$\begin{array}{ll}\text { Glossary ix } & \text { ix }\end{array}$

1 Introduction 13

1.1 Trends in Wide Bandwidth and High Dynamic Range ADCs . 15

1.2 Motivation and Objectives . . . . . . . . . . . . . . . . 16

1.3 Organization of the Thesis . . . . . . . . . . . . . 18

2 Continuous-Time Delta-Sigma Modulator 23

2.1 Ideal Delta-Sigma Modulator . . . . . . . . . . . . . . . . . . 23

2.1.1 System Overview . . . . . . . . . . . . . . . . . . 23

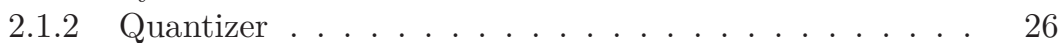

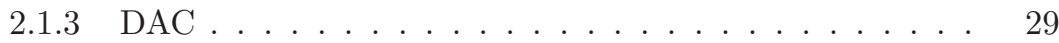

2.1.4 Loop Filter . . . . . . . . . . . . . . . . . . . . . 31

2.2 System-Level Non-Idealities . . . . . . . . . . . . . . . . . . 35

2.2 .1 Noise . . . . . . . . . . . . . . . . . . . . . 35

2.2 .2 Non-Linearity . . . . . . . . . . . . . . . . . . . . . 39

2.2 .3 Excess Loop Delay . . . . . . . . . . . . . . . . . . . . . 41

2.2 .4 Metastability . . . . . . . . . . . . . 47

2.3 Summary . . . . . . . . . . . . . . . . . . . 50

3 Continuous-Time Delta-Sigma Modulators at High Sampling Rates 55

3.1 System-Level Design . . . . . . . . . . . . . . . . . . 56

3.1.1 CT $\Delta \Sigma$ Modulator Design at High Sampling Rates . . . 56

3.1.2 Excess Loop Delay Compensation with an Active Amplifier 59

3.1.3 High-Speed Capacitive Feedforward CT $\Delta \Sigma$ Modulator $\quad 65$ 
3.2 Block-Level Design Requirements . . . . . . . . . . . . . . . . . 70

3.2 .1 Loop Filter . . . . . . . . . . . . . . . . . 71

3.2 .2 Quantizer .................. 77

3.2.3 Feedback DAC (DAC1) . . . . . . . . . . 86

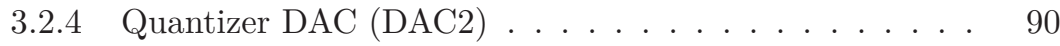

3.3 Conclusions ...................... . . 93

4 A $4 \mathrm{GHz}$ Continuous-Time $\Delta \Sigma$ ADC $\quad 99$

4.1 Introduction . . . . . . . . . . . . . . . . . . . . . 99

4.2 Implementation Details . . . . . . . . . . . . . . . . 100

4.2.1 CT $\Delta \Sigma$ ADC Architecture . . . . . . . . . . . . . 100

4.2.2 Quantizer Design and Timing Diagram of the Modulator 101

4.2 .3 Feedback DACs . . . . . . . . . . . . . . . . . 104

4.2.4 Operational Transconductance Amplifier . . . . . . . . . 106

4.2.5 Decimation Filter . . . . . . . . . . . . . . . . . . 107

4.3 Experimental Results . . . . . . . . . . . . . . . . 108

4.3.1 Measurement Setup . . . . . . . . . . . . . . . . 108

4.3.2 Measurement Results . . . . . . . . . . . . . 109

4.4 Conclusions . . . . . . . . . . . . . . . . . 119

5 A $2 \mathrm{GHz}$ Continuous-Time $\Delta \Sigma$ ADC with Dynamic Error Cor$\begin{array}{lr}\text { rection } & 123\end{array}$

5.1 Introduction . . . . . . . . . . . . . . . . . . . . . . 124

5.2 Dynamic Error Correction Techniques in $\Delta \Sigma$ Modulators . . . 129

5.2.1 The Error Switching Technique . . . . . . . . . . . . 134

5.3 Multi-Mode High-Speed $\Delta \Sigma$ ADC Design . . . . . . . . . . . . 137

5.4 Implementation Details . . . . . . . . . . . . . . . . . . . 139

5.4.1 Input Stage and the Loop Filter . . . . . . . . . . . . . 139

5.4 .2 Pulse Generator . . . . . . . . . . . . . . . . . . 141

5.5 Experimental Results . . . . . . . . . . . . . . . . . . 144

5.6 Conclusions ........................ . . . 147

6 Conclusions $\quad 151$

6.1 Benchmarking. . . . . . . . . . . . . . . . 152

6.2 Future Work . . . . . . . . . . . . . . . . 154

$\begin{array}{lr}\text { A Comparison of ADC Architectures } & 157\end{array}$

B Non-linearity of an Ideal Quantizer $\quad 161$

$\begin{array}{lc}\text { Summary } & 163\end{array}$

vi 
Samenvatting

List of publications

About the author

173

Acknowledgments

175 


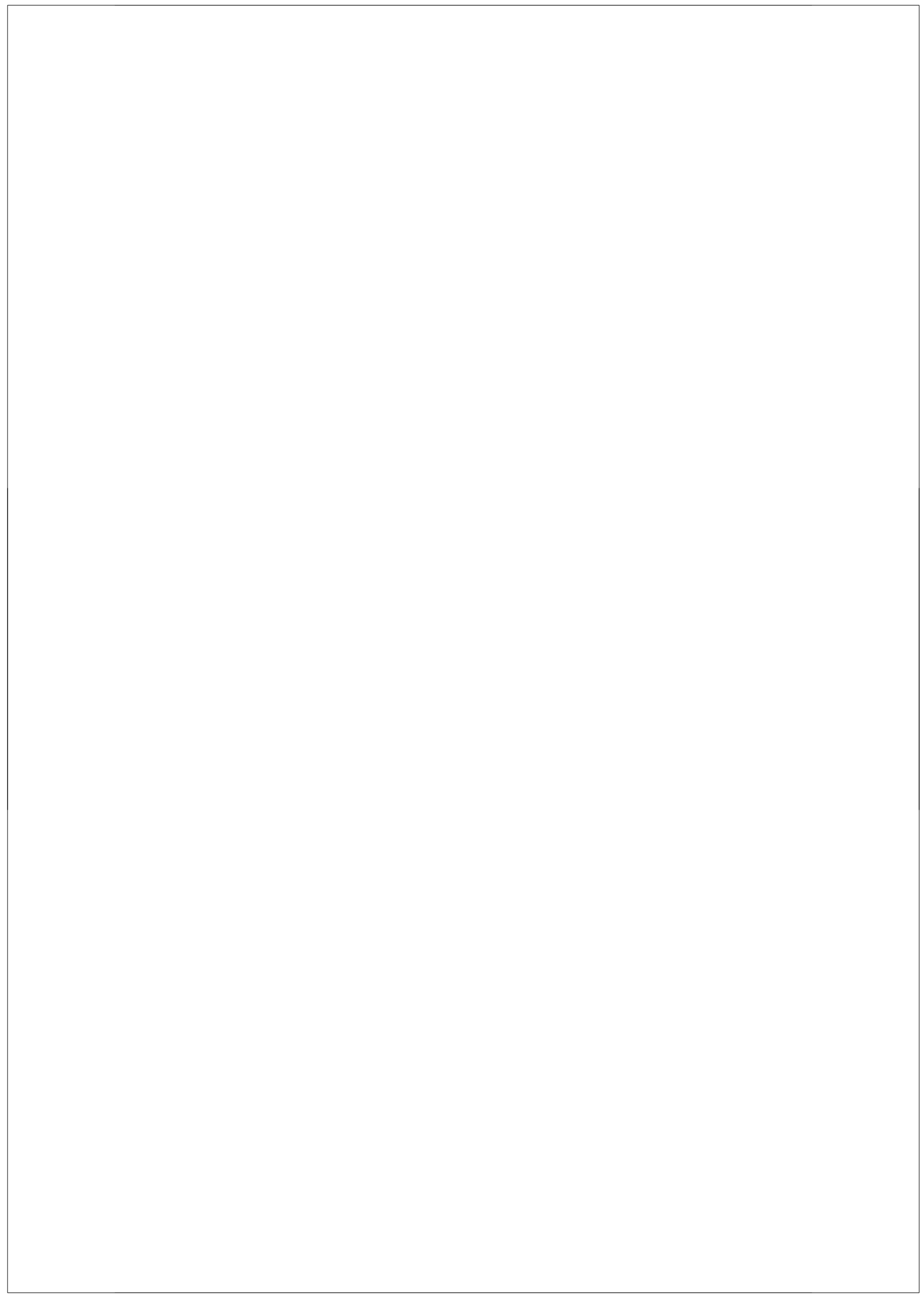




\section{Glossary}

\section{Acronyms}

$\Delta \Sigma \mathrm{M}$ delta-sigma modulator.

AAF anti-alias filtering.

ADC analog-to-digital converter.

BER bit-error-ratio.

BW bandwidth.

CM common mode.

CMOS complementary metal-oxide-semiconductor.

CT $\Delta \Sigma$ continuous-time delta-sigma.

DAC digital-to-analog converter.

DEM dynamic element matching.

DQS differential quad switching.

DR dynamic range.

DWA data weighted averaging.

ELD excess loop delay. 
FoM figure of merit.

GBW gain-bandwidth product.

HD harmonic distortion.

$\mathbf{H D}_{3} 3^{\text {rd }}$ order harmonic distortion.

I/O input/output.

ISI inter-symbol interference.

KCL Kirchhoff's current law.

LP-CMOS low-power complementary metal-oxide-semiconductor.

LSB least significant bit.

LVDS low voltage differential signaling.

NRZ non-return-to-zero.

NTF noise transfer function.

OSR oversampling ratio.

OTA operational transconductance amplifier.

PVT process, voltage, and temperature.

RMS root-mean-square.

RZ return-to-zero.

S\&H sample-and-hold.

SFDR spurious-free dynamic range.

SJNR signal-to-jitter-noise-ratio.

SNDR signal-to-noise-and-distortion ratio.

SNR signal-to-noise ratio. 
SQNR signal-to-quantization noise ratio.

STF signal transfer function.

THD total harmonic distortion.

UGBW unity-gain-bandwidth.

ZOH zero-order hold. 


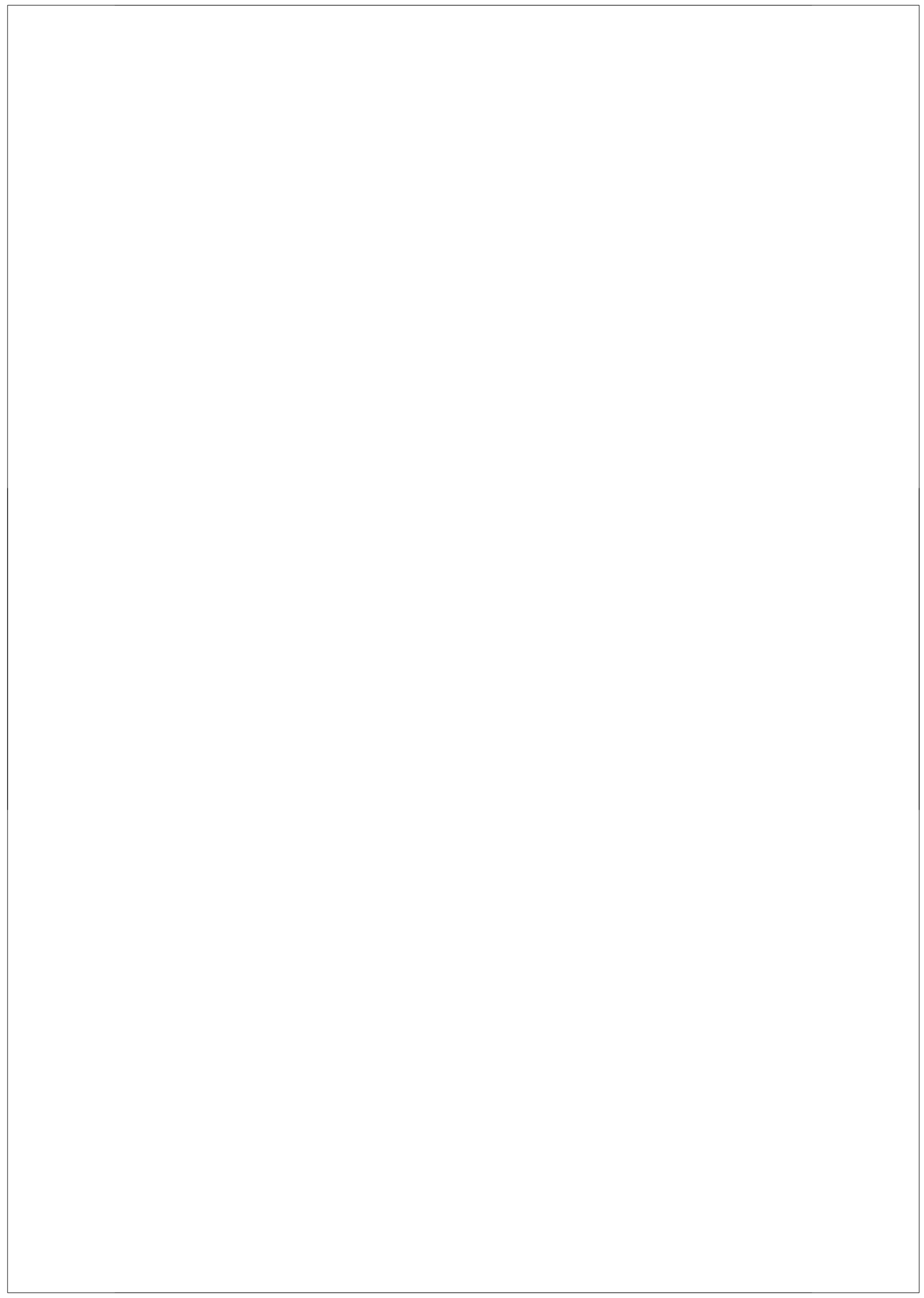




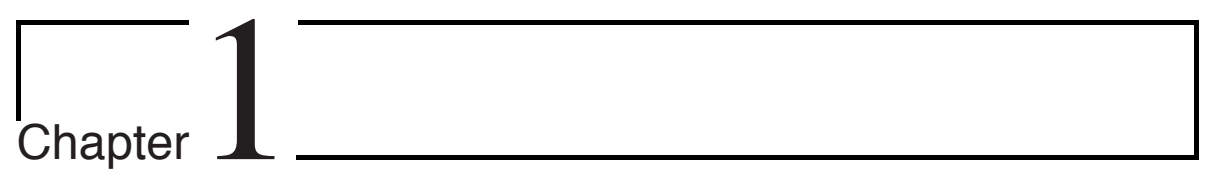

\section{Introduction}

Analog-to-digital converter developments are driven by the increasing demand for signal bandwidth and dynamic range in applications such as medical imaging, high-definition video processing and, in particular, wireline and wireless communications. Figure 1.1 shows a block diagram of a basic wireless receiver. It has three main building blocks: an RF front-end, an analog-to-digital converter (ADC) and a digital baseband processor. The role of the RF front-end is to filter, amplify the signals present at the antenna input and down-convert them to baseband. The ADC samples and digitizes the analog signals at the output of the RF front-end and outputs the results to the baseband processor. To achieve high data rates, wireless standards rely on advanced digital modulation techniques that can be advantageously implemented in baseband processors fabricated in nanometer-CMOS, which also motivates the development of ADCs in these technologies.

In modern wireless applications such as digital FM and LTE-advanced, the $\mathrm{ADC}$ receives a signal whose bandwidth can be as large as $100 \mathrm{MHz}[1-3]$. A wideband ADC which can capture such signals simplifies the design of the RF front-end, since the channel selection filters can then be implemented in the baseband processor. However, due to the limited filtering characteristic of the RF front-end, large unwanted signals (blockers) are often present at the input of the ADC. Therefore, the ADC should have a high dynamic range, often more than $70 \mathrm{~dB}$. Wide bandwidth and high dynamic range (DR) are thus important attributes of ADCs intended for high data-rate next-generation wireless applications.

Practically, Nyquist ADCs have been preferred for applications which target 


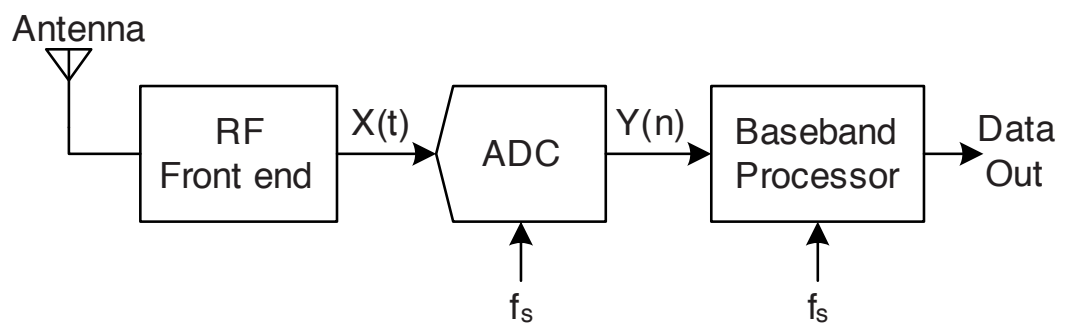

Figure 1.1 - A basic block diagram of a wireless receiver.

wide bandwidth, since the sampling frequency $\left(f_{s}\right)$ only has to be slightly higher than $2 \times B W$, where $B W$ is the bandwidth of the desired signal. A plot of dynamic range vs. bandwidth for various state-of-the-art ADCs with energy efficiency less than 1pJ/conv.-step. is shown in Fig. 1.2. As can be seen, many Nyquist ADCs achieve both wide bandwidths and high DR. A Nyquist ADC requires an input sampling circuit which is often implemented with a switchedcapacitor network. Achieving high DR, then requires low thermal noise, which in turn, leads to a large input capacitance. However, this must be preceded by an anti-aliasing filter and an input buffer capable of driving a large capacitance, which increases the complexity and power of the RF front-end.

Oversampled converters are very well suited for applications which require high dynamic range. In particular, a delta-sigma modulator $(\Delta \Sigma \mathrm{M})$, which trades time resolution for amplitude resolution, can achieve a high dynamic range with very good power efficiency (Fig. 1.2). The $\Delta \Sigma \mathrm{M}$ is one of the most promising converter architectures for exploiting the speed advantage of CMOS process technology. However, achieving a wide bandwidth with a $\Delta \Sigma \mathrm{M}$ requires a high-speed sampling frequency due to the large OSR $\left(f_{s}=2 \times O S R \times B W\right.$, where OSR is the oversampling ratio). The stability and power efficiency of the modulator at a high sampling rate, together with achieving a high dynamic range at the low supply voltages required by the nanometer-CMOS fabrication process, are important challenges that face the next generation of oversampled converters.

This thesis focuses on the design of wide-bandwidth and high dynamic range $\Delta \Sigma$ Ms that can bridge the bandwidth gap between Nyquist and oversampled converters. More specifically, this thesis describes the stability, the power efficiency and the linearity limits of $\Delta \Sigma \mathrm{Ms}$ aiming at a $\mathrm{GHz}$ sampling frequency. 


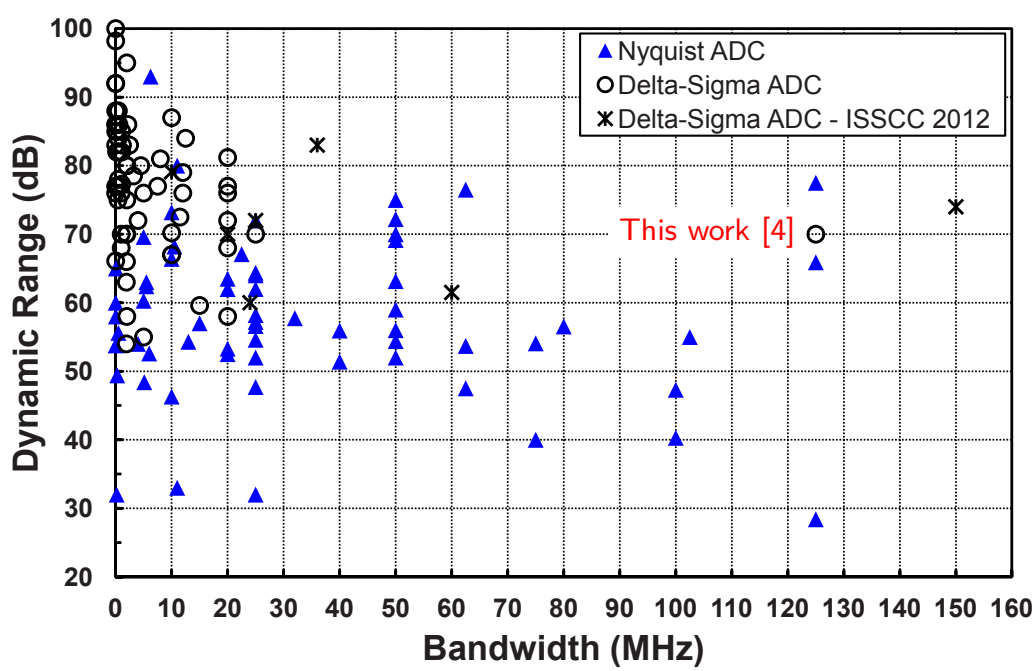

Figure 1.2 - Dynamic range vs. bandwidth of state-of-the-art ADCs with power efficiency less than $1 \mathrm{pJ} /$ conv.-step. The high speed $\mathrm{CT} \Delta \Sigma$ ADCs implemented in $\mathrm{nm}$-CMOS that have recently gained popularity are included to emphasize the developments in oversampled converters [5].

\subsection{Trends in Wide Bandwidth and High Dy- namic Range ADCs}

As shown in Fig. 1.2, Nyquist ADCs based on the pipeline architecture have achieved sampling speeds of up to $125 \mathrm{MHz}$ and dynamic ranges greater than $70 \mathrm{~dB}$ in standard CMOS [6-8]. To achieve higher sampling rates, a Bi-CMOS or SiGe Bi-CMOS process can be used at the cost of higher power consumption due to their higher supply voltages $(1.8 \mathrm{~V}-3.0 \mathrm{~V})[9,10]$. A further drawback of pipeline ADCs is that they typically rely on high-gain wideband residue amplifiers and/or complex calibration techniques to reduce gain errors [7-9], thus increasing their area and complexity.

Recently, Nyquist ADCs based on the successive approximation register (SAR) architecture have achieved signal bandwidths of up to $50 \mathrm{MHz}$ with 56 65dB DR and excellent power efficiency (<80fJ/conv.-step) [11-14]. Greater bandwidth can be achieved by using time-interleaving. However, the linearity of time-interleaved SAR ADCs is limited by gain, offset, and timing errors 
and so such ADCs also require extensive calibration [15]. Furthermore, time interleaving increases input capacitance and chip area, and thus places more demands on the input buffer [16].

By contrast, $\mathrm{CT} \Delta \Sigma \mathrm{ADCs}$ can have a simple resistive input that does not require the use of a power-hungry input buffer or an anti-aliasing filter, which further relaxes the requirements of the RF front-end. When implemented in CMOS, such ADCs have achieved signal bandwidths of up to $25 \mathrm{MHz}$ with a 70-80dB dynamic range and good power efficiency (<350fJ/conv.-step) $[17-$ 19]. Typical CT $\Delta \Sigma$ modulators employ a high-order loop filter with a multibit quantizer, which, for a $20 \mathrm{MHz}$ bandwidth, require sampling frequencies of $0.5-1 \mathrm{GHz}$ to achieve more than $70 \mathrm{~dB}$ of dynamic range. Assuming that the sampling frequency is proportional to the bandwidth, sampling frequencies of $2.5-5 \mathrm{GHz}$ will be then required to achieve bandwidths greater than $100 \mathrm{MHz}$. However, at $\mathrm{GHz}$ sampling rates, parasitic poles and quantizer latency can easily cause modulator instability.

$\mathrm{CT} \Delta \Sigma$ modulators with signal bandwidths up to $20-25 \mathrm{MHz}$ have been implemented in $90 \mathrm{~nm}-130 \mathrm{~nm}$ CMOS. The switching speed of an NMOS transistor in $45 \mathrm{~nm}$ CMOS is approximately 1.6x faster than in $90 \mathrm{~nm}$ CMOS and $2.7 \mathrm{x}$ faster than in $130 \mathrm{~nm}$ CMOS [20]. Implementing a $\Delta \Sigma$ modulator in $45 \mathrm{~nm} \mathrm{LP}$ CMOS is thus advantageous for circuits such as quantizers and DACs whose delay is important for stability. However, the dynamic range of circuits in $45 \mathrm{~nm}$ CMOS is limited by the low intrinsic gain and poor matching of the transistors $[21,22]$. The low operating supply $(1.1-1.0 \mathrm{~V})$ furthermore implies that cascaded stages are required to make gain in blocks such as an OTA or a quantizer. Therefore, the intrinsic speed of $45 \mathrm{~nm}$ LP CMOS cannot be fully utilized. To realize CT $\Delta \Sigma$ modulators with bandwidths greater than $100 \mathrm{MHz}$ in CMOS, innovations are still required at the system-level design. A comparison of ADC architectures targeting wide bandwidth $(\mathrm{BW}>100 \mathrm{MHz})$ and high dynamic range $(\mathrm{DR}>70 \mathrm{~dB})$ is presented in Appendix A.

\subsection{Motivation and Objectives}

The $\Delta \Sigma \mathrm{M}$ is an architecture which trades time resolution (signal bandwidth) for amplitude resolution, or in other words, dynamic range. Wide bandwidth and high dynamic range $\Delta \Sigma \mathrm{Ms}$ have received much attention since every new generation of CMOS process technology brings a speed advantage ${ }^{1}$. The fundamental limitations of a single-loop CT $\Delta \Sigma$ modulator targeting a wide band-

\footnotetext{
${ }^{1}$ Recently, high speed CT $\Delta \Sigma$ ADCs implemented in nm-CMOS have gained popularity [23-29].
} 
width and a high dynamic range define the scope of this thesis.

The aim of the research described in this thesis is to develop a wideband, high dynamic range $\Delta \Sigma M$ which demonstrates that an oversampled converter can also cover the application space where Nyquist ADCs are currently preferred. Furthermore, such a $\Delta \Sigma \mathrm{M}$ should also achieve state-of-the-art power efficiency. This quest is achieved by tackling the research question both at the system and circuit level.

A $\Delta \Sigma \mathrm{M}$ is a non-linear system, and often the design trade-offs are hidden behind complex system-level simulations. Therefore, system-level understanding of the modulator is required to find architectural solutions. The stability of a $\Delta \Sigma \mathrm{M}$ is a very important aspect of its design. As the sampling speed of the modulator increases to achieve more bandwidth, second order effects such as the limited unity gain bandwidth of amplifiers and the limited switching speed of the transistors start effecting the modulator's stability. One of the main research goals of this thesis is to find system level solutions that enable the design of a wide bandwidth, high dynamic range modulator with state-of-the-art power efficiency.

Theoretically, it is possible to design a stable $\Delta \Sigma \mathrm{M}$ for any given specification [30]. However, practical limitations at the circuit level define the possible solutions that can be implemented. For example, the limited speed of the transistors introduces excess loop delay (ELD) which degrades the stability of the modulator, and at GHz sampling frequencies, ELD limits the performance. Such practical limitations might be solved by dissipating more power, although this does not prove that a stable $\Delta \Sigma \mathrm{M}$ with desired specifications can be implemented. As a second objective of this thesis, we explore the circuit-level design techniques to assist the proposed system-level design solutions and push the design boundary of the oversampled converters in terms of dynamic range, bandwidth, linearity, and power efficiency.

To demonstrate the feasibility of the ideas and approaches presented in this thesis, we have designed and implemented a wideband $\mathrm{CT} \Delta \Sigma$ with a bandwidth (BW) greater than $100 \mathrm{MHz}$ and a dynamic range above $70 \mathrm{~dB}$ in $\mathrm{nm}-\mathrm{CMOS}$. This is achieved by using a low oversampling ratio and multi-bit architecture. The performance of a multi-bit $\mathrm{CT} \Delta \Sigma$ is often limited by the dynamic errors at GHz sampling rates, and the correction/calibration techniques that are applicable are bounded by the stability requirements. To overcome these limitations, we have implemented a dynamic error correction technique which not only experimentally quantifies the level of dynamic errors but also improves the dynamic performance of the modulator. 


\subsection{Organization of the Thesis}

Chapter 2 starts with a brief description of an ideal single-loop $\Delta \Sigma \mathrm{M}$. The building blocks of the modulator are analyzed and their characteristic properties are discussed to provide a basic understanding of the modulator's operation. The stability of the $\Delta \Sigma \mathrm{M}$ is discussed and the relation between this and the main building blocks is presented. Moreover, this chapter discusses the system-level non-idealities in a $\Delta \Sigma \mathrm{M}$ such as noise, nonlinearity, metastability and ELD. The understanding of the system-level non-idealities is especially important to achieve the optimum performance for a given $\Delta \Sigma \mathrm{M}$ architecture.

Chapter 3 focuses on the design of $\mathrm{CT} \Delta \Sigma$ modulators aiming at $\mathrm{GHz}$ sampling frequencies. The system-level non-idealities discussed in Chapter 2 pose a major limitation at these frequencies, and limit the possible architectural implementations. In this chapter, we present the system-level trade-offs in a single-loop $\Delta \Sigma \mathrm{M}$ and propose a $3^{r d}$ order multi-bit $\Delta \Sigma \mathrm{M}$ which can achieve an $80 \mathrm{~dB}$ signal-to-quantization noise ratio (SQNR) in a $125 \mathrm{MHz} \mathrm{BW}$ with a sampling rate of $4 \mathrm{GHz}$. Mitigating ELD and metastability are crucial to meet the target sampling rate, therefore we present a high speed modulator architecture which overcomes the limitation of the summation amplifier present in high speed modulators, and improves its power efficiency. Furthermore, we present the block-level design requirements of the proposed architecture. Each building block is analyzed based on its most important non-ideality and blocklevel specifications are listed.

Chapter 4 describes the implementation details of a $4 \mathrm{GHz} \mathrm{CT} \Delta \Sigma$ ADC which uses the high-speed modulator architecture proposed in Chapter 3. The ADC is implemented in 45nm-LP CMOS and achieves a $70 \mathrm{~dB}$ DR and $-74 \mathrm{dBFS}$ total harmonic distortion (THD) in a $125 \mathrm{MHz}$ BW. Since the clocking scheme of the quantizer and feedback DACs is crucially important for the stability of the modulator, this chapter presents a detailed timing diagram of the modulator. The implemented ADC is characterized by using a custom measurement setup, and the detailed measurement results are presented particularly focusing on the jitter performance of the ADC.

Chapter 5 explains a $2 \mathrm{GHz}$ CT $\Delta \Sigma$ ADC where dynamic errors of its multibit digital-to-analog converter (DAC) are masked by using an error switching (ES) scheme at the virtual ground node of the first integrator. This technique prevents the loop filter from processing the dynamic errors in the feedback DAC and improves the signal-to-noise ratio (SNR), signal-to-noise-and-distortion ratio (SNDR), and THD of the modulator. This chapter also explains the design and implementation of a multi-mode version of the high-speed architecture presented in Chapter 4. Furthermore, a high-speed error sampling switch driver 
is discussed and detailed measurement results are presented.

Finally, Chapter 6 concludes this work and suggests future research directions based on the insight gained during this research.

\section{References}

[1] L. Breems, R. Rutten, R. van Veldhoven, and G. van der Weide, "A 56 $\mathrm{mW}$ continuous-time quadrature cascaded $\Sigma \Delta$ modulator with $77 \mathrm{~dB}$ DR in a near zero-IF $20 \mathrm{MHz}$ band," in Solid-State Circuits, IEEE Journal of, vol. 42, no. 12, pp. 2696-2705, Dec. 2007.

[2] S. Abeta, "Toward LTE commercial launch and future plan for LTE enhancements (LTE-Advanced)," in Communication Systems (ICCS), 2010 IEEE International Conference on, Nov. 2010, pp. 146-150.

[3] S. Parkvall, A. Furuskär, and E. Dahlman, "Evolution of LTE toward IMT-Advanced," in Communications Magazine, IEEE, vol. 49, no. 2, pp. 84-91, Feb. 2011.

[4] M. Bolatkale, L. Breems, R. Rutten, and K. Makinwa, "A 4GHz CT $\Delta \Sigma$ ADC with $70 \mathrm{~dB}$ DR and -74dBFS THD in $125 \mathrm{MHz}$ BW ," in Solid-State Circuits Conference, 2011. ISSCC 2011. Digest of Technical Papers. IEEE International, Feb. 2011, pp. 470-472.

[5] B. Murmann, ADC Performance Survey 1997-2012 [Online]. Available: http://www.stanford.edu/ murmann/adcsurvey.html.

[6] B.-G. Lee, B.-M. Min, G. Manganaro, and J. Valvano, "A 14-b 100-MS/s pipelined ADC with a merged SHA and first MDAC," in Solid-State Circuits, IEEE Journal of, vol. 43, no. 12, pp. 2613-2619, Dec. 2008.

[7] H. Van de Vel et al., "A 1.2-V 250-mW 14-b 100-MS/s digitally calibrated pipeline ADC in 90-nm CMOS," in Solid-State Circuits, IEEE Journal of, vol. 44, no. 4, pp. 1047-1056, Apr. 2009.

[8] S. Devarajan et al., "A 16-bit, $125 \mathrm{MS} / \mathrm{s}, 385 \mathrm{~mW}, 78.7$ dB SNR CMOS pipeline ADC," in Solid-State Circuits, IEEE Journal of, vol. 44, no. 12, Dec. 2009.

[9] A. Ali et al., "A 16-bit 250-MS/s IF sampling pipelined ADC With background calibration," in Solid-State Circuits, IEEE Journal of, vol. 45, no. 12 , pp. 2602-2612, Dec. 2010. 
[10] R. Payne et al., "A 16-Bit 100 to 160 MS/s SiGe BiCMOS pipelined ADC With 100 dBFS SFDR," in Solid-State Circuits, IEEE Journal of, vol. 45, no. 12, pp. 2613-2622, Dec. 2010.

[11] C.-C. Liu, S.-J. Chang, G.-Y. Huang, and Y.-Z. Lin, "A 10-bit 50-MS/s SAR ADC with a monotonic capacitor switching procedure," in Solid-State Circuits, IEEE Journal of, vol. 45, no. 4, pp. 731-740, Apr. 2010.

[12] C. Lee and M. Flynn, "A 12b 50MS/s 3.5mW SAR assisted 2-stage pipeline ADC," in VLSI Circuits (VLSIC), 2010 IEEE Symposium on, June 2010, pp. 239-240.

[13] Y. Zhu et al., "A 10-bit 100-MS/s reference-free SAR ADC in $90 \mathrm{~nm}$ CMOS," in Solid-State Circuits, IEEE Journal of, vol. 45, no. 6, pp. 11111121, June 2010.

[14] M. Yoshioka, K. Ishikawa, T. Takayama, and S. Tsukamoto, "A 10b $50 \mathrm{MS} / \mathrm{s} 820 \mu \mathrm{W}$ SAR ADC with on-chip digital calibration," in Solid-State Circuits Conference Digest of Technical Papers (ISSCC), 2010 IEEE International, Feb. 2010, pp. 384-385.

[15] S. Louwsma, A. van Tuijl, M. Vertregt, and B. Nauta, "A 1.35 GS/s, 10b, $175 \mathrm{~mW}$ time-interleaved AD converter in 0.13 $\mu \mathrm{m}$ CMOS," in Solid-State Circuits, IEEE Journal of, vol. 43, no. 4, pp. 778-786, Apr. 2008.

[16] B. Ginsburg and A. Chandrakasan, "Highly interleaved 5-bit, 250MSample/s, 1.2-mW ADC with redundant channels in 65-nm CMOS," in Solid-State Circuits, IEEE Journal of, vol. 43, no. 12, pp. 2641-2650, Dec. 2008.

[17] G. Mitteregger et al., "A 20-mW 640-MHz CMOS continuous-time ADC With 20-MHz signal bandwidth, 80-dB dynamic range and 12-bit ENOB," in Solid-State Circuits, IEEE Journal of, vol. 41, no. 12, pp. 2641-2649, Dec. 2006.

[18] M. Park and M. Perrott, "A 78 dB SNDR $87 \mathrm{~mW} 20 \mathrm{MHz}$ bandwidth continuous-time $\Delta \Sigma$ ADC with VCO-based integrator and quantizer implemented in $0.13 \mu \mathrm{m}$ CMOS," in Solid-State Circuits, IEEE Journal of, vol. 44, no. 12, pp. 3344-3358, Dec. 2009.

[19] J. Kauffman, P. Witte, J. Becker, and M. Ortmanns, "An 8mW 50MS/s $\mathrm{CT} \Delta \Sigma$ modulator with $81 \mathrm{~dB}$ SFDR and digital background DAC linearization," in Solid-State Circuits Conference Digest of Technical Papers (ISSCC), 2011 IEEE International, Feb. 2011, pp. 472-474. 
[20] International Technology Roadmap for Semiconsuctors (ITRS) 2001, 2003, 2007, 2009 Editions. Available: http://www.itrs.net/reports.html. [Online]. Available: Available: http://www.itrs.net/reports.html

[21] M. Pelgrom, H. Tuinhout, and M. Vertregt, "Transistor matching in analog CMOS applications," in Electron Devices Meeting, 1998. IEDM '98 Technical Digest., International, Dec. 1998.

[22] M. Vertregt, "The analog challenge of nanometer CMOS," in Electron Devices Meeting, 2006. IEDM '06. International, Dec. 2006.

[23] J. Harrison et al., "An LC bandpass $\Delta \Sigma$ ADC with 70dB SNDR over $20 \mathrm{MHz}$ bandwidth using CMOS DACs," in Solid-State Circuits Conference, 2012. ISSCC 2012. Digest of Technical Papers. IEEE International, Feb. 2012, pp. 146-147.

[24] J. Chae, H. Jeong, G. Manganaro, and M. Flynn, "A 12mW low-power continuous-time bandpass $\Delta \Sigma$ with $58 \mathrm{~dB}$ SNDR and $24 \mathrm{MHz}$ bandwidth at 200MHz IF," in Solid-State Circuits Conference, 2012. ISSCC 2012. Digest of Technical Papers. IEEE International, Feb. 2012, pp. 148-149.

[25] H. Shibata et al., "A DC-to- $1 \mathrm{GHz}$ tunable RF $\Delta \Sigma$ ADC achieving $\mathrm{DR}=74 \mathrm{~dB}$ and $\mathrm{BW}=150 \mathrm{MHz}$ at $f_{0}=450 \mathrm{MHz}$ using $550 \mathrm{~mW}$," in SolidState Circuits Conference, 2012. ISSCC 2012. Digest of Technical Papers. IEEE International, Feb. 2012, pp. 150-151.

[26] K. Reddy et al., "A 16mW 78dB-SNDR 10MHz-BW CT- $\Delta \Sigma$ ADC using residue-canceling VCO-based quantizer," in Solid-State Circuits Conference, 2012. ISSCC 2012. Digest of Technical Papers. IEEE International, Feb. 2012, pp. 152-153.

[27] P. Witte et al., "A 72dB-DR $\Delta \Sigma$ CT modulator using digitally estimated auxiliary DAC linearization achieving $88 \mathrm{fJ} /$ conv in a $25 \mathrm{MHz} \mathrm{BW}$," in Solid-State Circuits Conference, 2012. ISSCC 2012. Digest of Technical Papers. IEEE International, Feb. 2012, pp. 154-155.

[28] P. Shettigar and S. Pavan, "A 15mW 3.6GS/s CT- $\Delta \Sigma$ ADC with $36 \mathrm{MHz}$ bandwidth and 83 DR in 90nm CMOS," in Solid-State Circuits Conference, 2012. ISSCC 2012. Digest of Technical Papers. IEEE International, Feb. 2012, pp. 156-157.

[29] V. Srinivasan et al., "A 20mW 61dB SNDR (60MHz BW) 1b $3^{\text {rd-order }}$ continuous-time bandpass delta-sigma modulator clocked at $6 \mathrm{GHz}$ in $45 \mathrm{~nm}$ 
CMOS," in Solid-State Circuits Conference, 2012. ISSCC 2012. Digest of Technical Papers. IEEE International, Feb. 2012, pp. 158-159.

[30] S. Norsworthy, R. Schreier, and G. Temes, Delta-Sigma Data Converters (Theory, Design, and Simulation). John Wiley and Sons, Inc, 1996. 


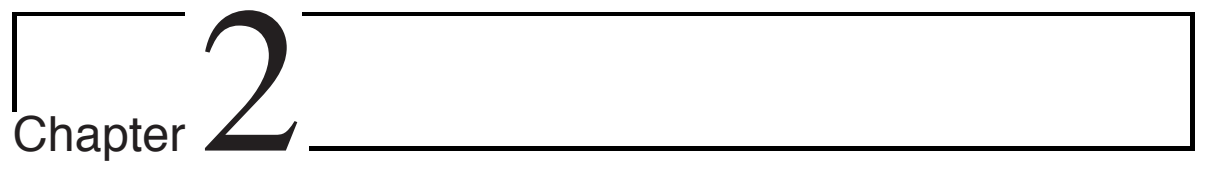

\section{Continuous-Time Delta-Sigma Modulator}

This chapter starts with a brief explanation of the operation of an ideal singleloop continuous-time delta-sigma $(\mathrm{CT} \Delta \Sigma)$ modulator and describes its major building blocks, i.e. the loop filter, quantizer and digital-to-analog converter (DAC). In Section 2.2, we introduce the system-level non-idealities that limit the performance of such a modulator. Finally, we will illustrate the effect of system-level non-idealities on the key performance metrics of the modulator: its signal-to-noise ratio (SNR), spurious-free dynamic range (SFDR), and sampling speed $\left(f_{s}\right)$.

\subsection{Ideal Delta-Sigma Modulator}

\subsubsection{System Overview}

A basic model of a single-loop delta-sigma modulator $(\Delta \Sigma \mathrm{M})$ is shown in Fig. 2.1a. It has three main building blocks: a quantizer, a DAC and a loop filter. Although, a $\Delta \Sigma \mathrm{M}$ is a non-linear feedback system, it can be approximated by a linear model (Fig. 2.1b) in order to develop a basic understanding of its behavior. The quantizer can be modeled as an error source which has a white noise spectrum. The DAC can be modeled as a unity gain stage, and the 


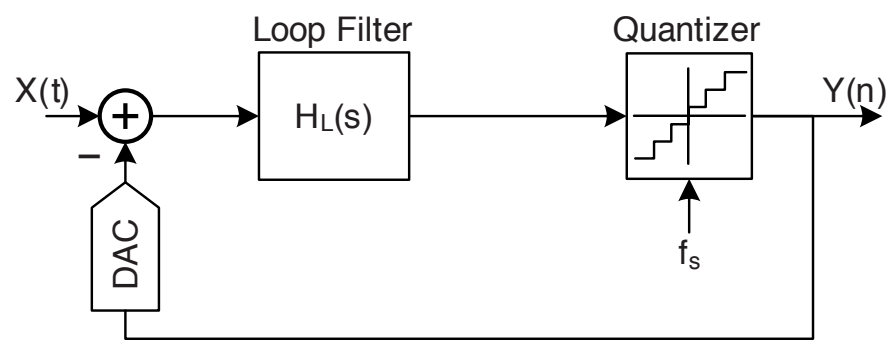

(a)

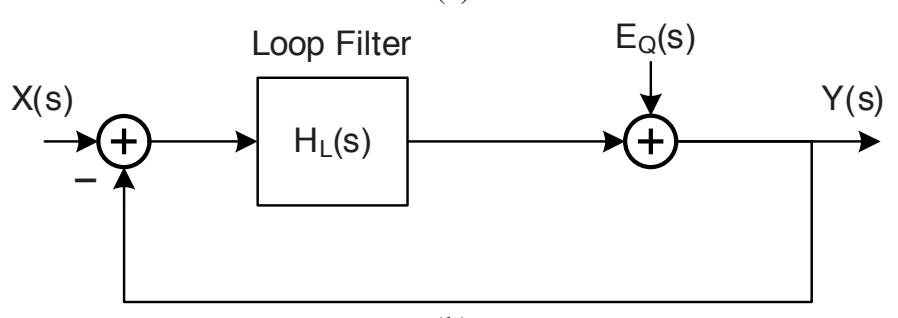

(b)

Figure 2.1 - A basic single-loop continuous-time $\Delta \Sigma$ modulator (a), and its linear model (b).

transfer function of the $\Delta \Sigma \mathrm{M}$ is expressed as:

$$
\begin{aligned}
Y(s) & =X(s) \cdot \frac{H_{L}(s)}{1+H_{L}(s)}+E_{Q}(s) \cdot \frac{1}{1+H_{L}(s)} \\
& =X(s) \cdot S T F(s)+E_{Q}(s) \cdot N T F(s),
\end{aligned}
$$

where $X$ is the input signal, $E_{Q}$ is the quantization noise, and $H_{L}$ is the transfer function of the loop filter. The input signal and quantization noise are subject to different transfer functions, which are known as the signal transfer function (STF) and the noise transfer function (NTF), respectively. Figure 2.2 presents the STF and NTF of a $3^{\text {rd }}$ order feedforward $\Delta \Sigma \mathrm{M}$. When $H_{L}$ consists of a cascade of integrators, then the quantization noise is high-pass filtered and is thus attenuated, or in other words, shaped in the band of interest due to the gain provided by the loop filter. On the other hand, the input signals located in the band of interest are processed without any attenuation.

In a $\mathrm{CT} \Delta \Sigma$ modulator, the sampling takes place at the output of the loop filter. These sampled values can be obtained from a discrete-time equivalent $\left(H_{L, d t}(z)\right)$ of the continuous-time loop filter $\left(H_{L}(s)\right)$, which can be obtained 


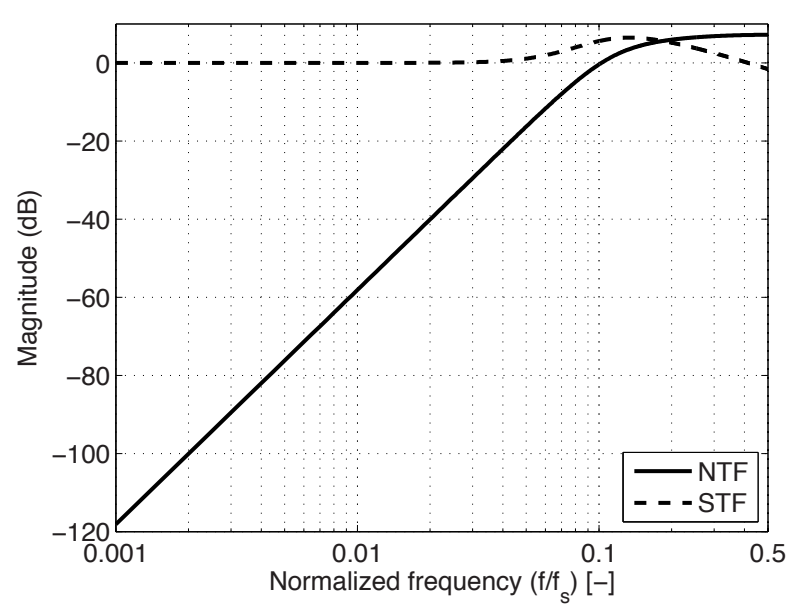

Figure 2.2 - Signal and noise transfer function of a feedforward $3^{\text {rd }}$ order CT $\Delta \Sigma$ modulator.

by using the impulse-invariant transformation [1]. This will be explained in more detail in Section 2.2.3.

One of the most important advantages of a $\mathrm{CT} \Delta \Sigma$ modulator is its inherent anti-alias filtering (AAF). In a Nyquist analog-to-digital converter (ADC), signals at $n \cdot f_{s} \pm f_{b}$ alias to $f_{b}<B W$ due to the sampling and cannot be distinguished from the signals present at $f<B W$. In a $\mathrm{CT} \Delta \Sigma$ modulator, however, the sampling takes place at the output of the loop filter and so signals which might alias are low-pass filtered by the loop filter. Therefore, the inherent AAF simplifies the filtering required in the analog front end. The aliasing component of a signal with frequency $\left(\omega=2 \pi\left(n \cdot f_{s} \pm f_{b}\right)\right)$ is scaled by the response of AAF, which is expressed for the single-loop $\Delta \Sigma \mathrm{M}$ as [2]:

$$
A A F(\omega)=\frac{H_{L}(j \omega)}{H_{L, d t}\left(e^{j \omega T_{s}}\right)},
$$

where $H_{L}, H_{L, d t}$ are the continuous-time and discrete-time equivalent of the loop filter, respectively. Fig. 2.3 shows the gain response of the $3^{\text {rd }}$ order modulator (Section 2.1.4) with AAF around $\left(f_{s} \pm f_{b}\right)$. For higher-order modulators, a more aggressive AAF roll-off can be achieved [3].

As mentioned before, a $\Delta \Sigma \mathrm{M}$ is a high-order feedback system and so it is not necessarily stable. A complete analysis of its stability is not trivial since the quantizer is a non-linear element. In most practical cases, the stability of a 


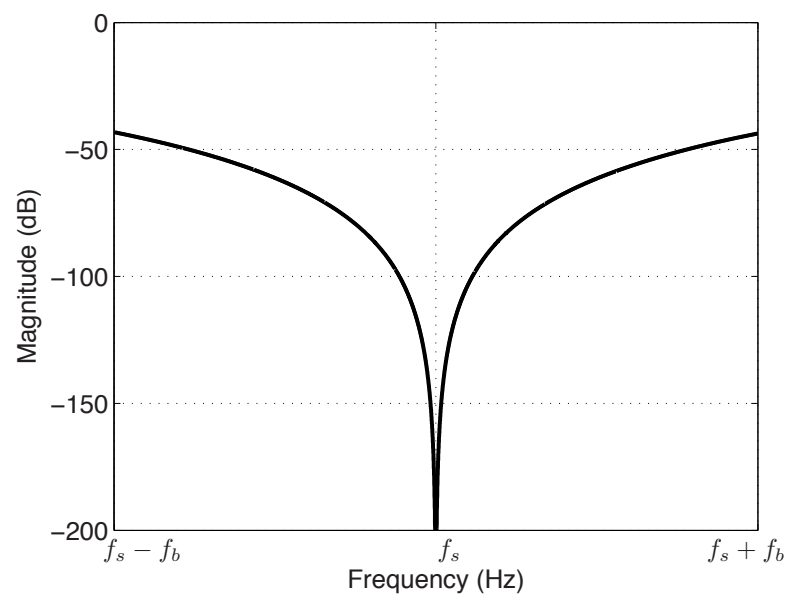

Figure 2.3 - Antialias filtering effect of a $3^{\text {rd }}$ order feedforward CT $\Delta \Sigma$ modulator.

$\Delta \Sigma \mathrm{M}$ is verified by computer simulations $[4,5]$. However, the building blocks of a modulator can be modeled to a certain extent, which reveals the link between its stability and the characteristics of each building block. Then, it is possible to establish a basic understanding of the stability of a $\Delta \Sigma \mathrm{M}$ and analyze how each building block effects the operation of the modulator. Therefore, in the following sub-sections, the main building blocks of an ideal single loop $\Delta \Sigma \mathrm{M}$ are described in more detail.

\subsubsection{Quantizer}

The quantizer converts the output of the loop filter to digital, and is the only non-linear element of the ideal modulator. The linearized transfer function can be expressed as:

$$
Y\left(n \cdot T_{s}\right)=G \cdot X\left(n \cdot T_{s}\right)+E_{Q}\left(n \cdot T_{s}\right),
$$

where $G$ is the gain of the quantizer and $E_{Q}$ is the quantization error. An example of the transfer function of a two bit quantizer with a unit-step size $(\Delta=1)$ is shown in Fig. 2.4a. The maximum input amplitude is defined as $A_{m}=2^{B-1}$ where B is the number of bits of the quantizer. For an input signal lower than $A_{m}$, the quantizer is not overloaded and the quantization error is bounded between $\pm \Delta / 2$ (Fig. 2.4b). For a uniformly distributed quantization 
noise, its power is expressed as [4]:

$$
E_{Q, r m s}^{2}=\Delta^{2} / 12
$$

For input frequencies that are a rational fraction of the sampling frequency, a single-bit quantizer exhibits phase uncertainty [6]. Fig. 2.5 shows the output of a single-bit quantizer (indicated by the arrows) for an input signal at $f_{s} / 4$. If the signal crosses zero between two consecutive samples of the quantizer, the output of the quantizer will only toggle at the next sampling instance. For an input signal at $f_{s} / 4$, the single-bit quantizer has a $\pm \pi / 4$ phase uncertainty. In other words, shifting the input signal by $\pm \pi / 4$ results in exactly the same output. Therefore, the simple gain model of the quantizer can be extended to accommodate the phase uncertainty. The linear gain $(G)$ in $(2.3)$ is replaced by $G \cdot e^{s \theta}$, where $\theta$ is the phase uncertainty.

The non-linear behavior of the quantizer has a significant effect on the stability of the modulator. The phase uncertainty of a single-bit $\Delta \Sigma \mathrm{M}$ causes idle-patterns at the output of the modulator, which can cause instability. During the design of a single-bit modulator, therefore, the phase uncertainty must be taken into account to ensure a stable modulator. This effect is less dominant in a multi-bit quantizer. The phase uncertainty of a quantizer can be neglected for $\mathrm{B}>3[7]$.

In addition to the phase uncertainty, the uniformly distributed quantization noise assumption does not hold for a noiseless sine-wave input. The quantization error and the input signal will be highly correlated and harmonic distortion will be present at the output of the quantizer. This effect is especially dominant in a single-bit quantizer. For example, for an input signal at $f_{\text {in }} \ll f_{s}$, the output of the quantizer can be approximated as a square wave at $f_{\text {in }}$ which has odd harmonics of the input frequency. A detailed analysis of the nonlinearity of an ideal quantizer is presented in Appendix B.

Figure 2.6 shows the harmonic distortion and intermodulation of an ideal quantizer. For a $3^{\text {rd }}$ order harmonic distortion $\left(\mathrm{HD}_{3}\right)$ simulation, the input signal is set to $f_{\text {in }}=0.15 \times f_{s}$, and for an $\mathrm{IM}_{3}$ simulation the input is set to $f_{\text {in }} \pm \Delta f$ where $\Delta f=f s / 32$ for a two-tone input signal. The maximum resolution of the quantizer is set to five bits because higher resolution is not of practical interest. The simulation results are in agreement with the theoretical calculations (B.4,B.5). As the resolution of the quantizer increases the $\mathrm{HD}_{3}$ and $\mathrm{IM}_{3}$ improve. As a result, the nonlinearity of the quantizer can be neglected for $\mathrm{B}>3$ since the gain of the loop filter will further suppress these tones. Moreover, the nonlinearity of other blocks is often higher than the nonlinearity of the multi-bit quantizer assuming that the slices of the quantizer do not have any mismatch. 


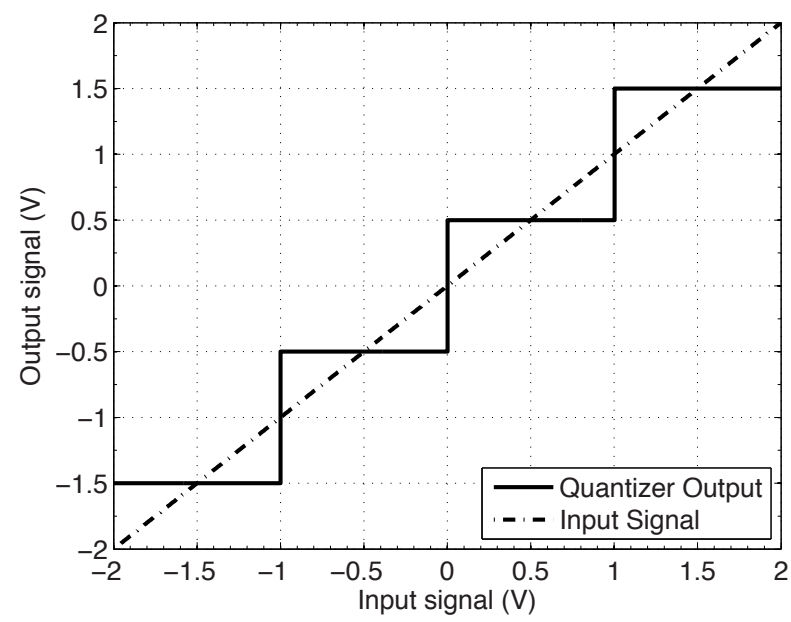

(a)

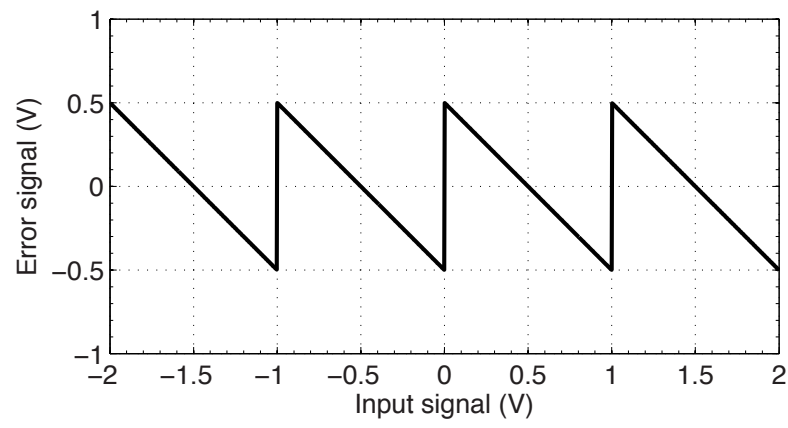

(b)

Figure 2.4 - The transfer function of a two bit quantizer (a), and the quantization error $E_{Q}(\mathrm{~b})$. 


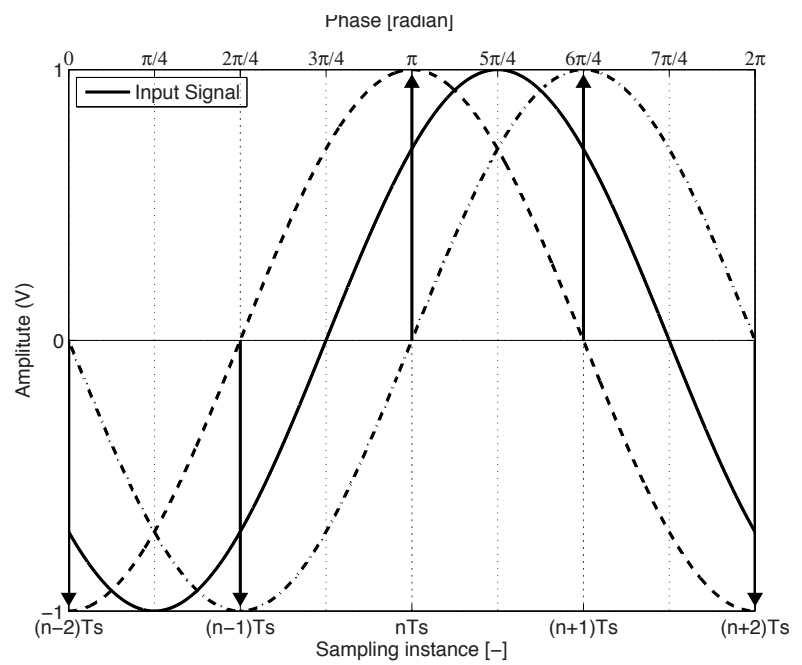

Figure 2.5 - Phase uncertainty of a single-bit quantizer for a sinewave at $f_{s} / 4$.

On the other hand, there is always some noise at the input of the quantizer in a practical implementation. The additional noise de-correlates the distortion tones generated by the quantizer and improves the $\mathrm{HD}_{3}$ and $\mathrm{IM}_{3}$ [8]. To illustrate this effect, a uniformly distributed noise with an amplitude of $1 L S B$ is added at the input of the quantizer and the input amplitude is reduced to prevent the overloading of the quantizer. The simulation results are shown in Fig. 2.7. The SNR diminishes due to the additional noise, but $\mathrm{HD}_{3}$ and $\mathrm{IM}_{3}$ improve by more than $10 \mathrm{~dB}$. Therefore, a quantizer will exhibit fewer distortion tones when used in a $\Delta \Sigma \mathrm{M}$ due to the thermal noise present in the modulator.

Furthermore, the harmonics introduced by the quantizer are attenuated by the loop gain provided by the $\Delta \Sigma \mathrm{M}$. However, the tones introduced by a singlebit quantizer cannot be ignored in low-order modulators. As the resolution of the quantizer increases, the $\mathrm{HD}_{3}$ and $\mathrm{IM}_{3}$ introduced by the quantizer become less dominant (Section 2.2).

\subsubsection{DAC}

The DAC is often the only block placed in the feedback of the modulator. In most cases, it uses the same number of levels as the quantizer and it converts the output of the quantizer into an analog signal by using voltage or current sources connected to the input of the loop filter. Furthermore, it introduces a 


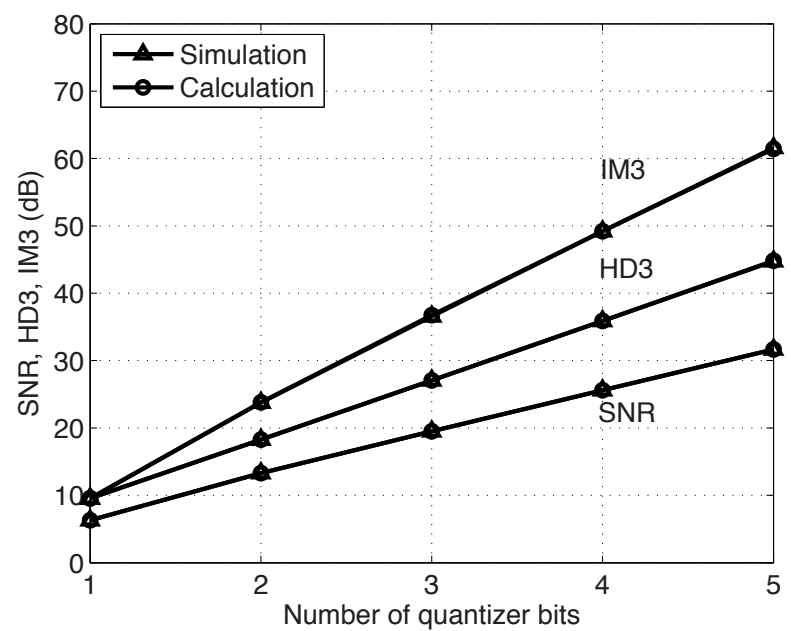

Figure 2.6 - Signal-to-noise ratio (SNR), $3^{\text {rd }}$ order harmonic distortion $\left(\mathrm{HD}_{3}\right)$, and $3^{\text {rd }}$ order intermodulation $\left(\mathrm{IM}_{3}\right)$ of a quantizer.

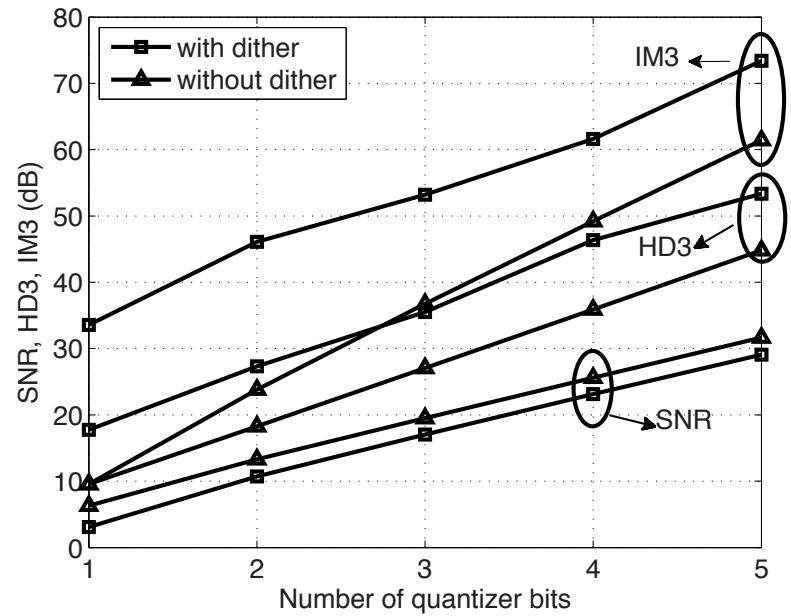

Figure 2.7 - Signal-to-noise ratio, $3^{\text {rd }}$ order harmonic distortion, and $3^{\text {rd }}$ order intermodulation of a quantizer with additional input noise. 


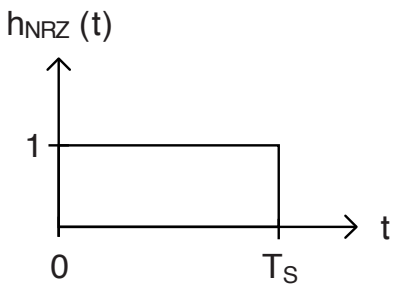

(a)

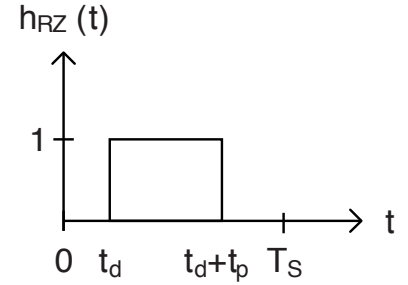

(b)

Figure 2.8 - Non-Return-to-Zero (NRZ) DAC impulse response (a), and Return-to-Zero (RZ) DAC impulse response (b).

zero-order hold $(\mathrm{ZOH})$ function to the feedback of the modulator. The DAC output waveform can have different shapes depending on the implementation requirements. Two commonly used DAC waveforms which are suitable for high-speed $\Delta \Sigma \mathrm{Ms}$ are illustrated in Fig. 2.8. A non-return-to-zero (NRZ) DAC holds the value of the digital data for one clock period $\left(T_{s}\right)$, whereas a return-to-zero (RZ) DAC uses only a fraction of the clock period. To analyze the stability of the modulator, the transfer function of the DAC waveforms (Fig. 2.8) can be expressed as:

$$
\begin{aligned}
H_{D A C, N R Z}(s) & =\frac{1-e^{-s T_{s}}}{s} \\
H_{D A C, R Z}(s) & =\frac{e^{-s t_{d}} \cdot\left(1-e^{-s t_{p}}\right)}{s},
\end{aligned}
$$

where $t_{d}$ is the delay and $t_{p}$ is the pulse width of the RZ DAC. The DAC introduces a frequency-dependent amplitude and phase response as shown in Fig. 2.9. The phase shift of an NRZ DAC is $90^{\circ}$ at $f_{s} / 2$, which must be taken into account when considering the stability of the modulator.

\subsubsection{Loop Filter}

The loop filter provides gain for the modulator which attenuates the quantization errors in the band of interest. It can usually be approximated as being a cascade of ideal integrator stages. Thus the transfer function of an $N^{\text {th }}$ order loop filter can be expressed as:

$$
H_{L}(s)=\left(\frac{1}{s}\right)^{N} .
$$



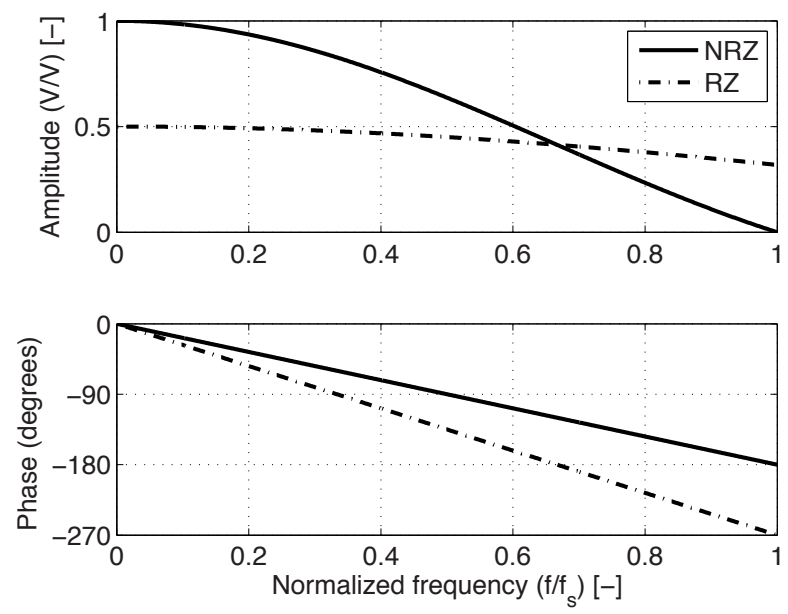

Figure 2.9 - Laplace transform of a Non-Return-to-Zero (NRZ) DAC (a) and Return-to-Zero (RZ) DAC with $t_{p}=0.5 T_{s}$ and $t_{d}=0.5 T_{s}(\mathrm{~b})$.

A higher-order loop filter achieves more aggressive noise shaping but at the cost of degrading the stability. An often-mentioned stability criterion for a $\Delta \Sigma M$ is that it generates bounded outputs for bounded input signals [4, 5, 9].

For a zero-input signal, the output of the multi-bit modulator (Fig. 2.1a) will be $(\ldots,+L S B,-L S B,+L S B,-L S B, \ldots)$, the average value of the output will be zero, and the frequency of oscillation will be $f_{s} / 2$. In other words, a stable $\Delta \Sigma \mathrm{M}$ exhibits tones at $f_{s} / 2$ for a bounded input signal.

To achieve controlled oscillations at $f_{s} / 2$, the gain and phase of the closedloop transfer function of the modulator at $f_{s} / 2$ must be " 1 " and " $2 \pi$ ", respectively which is also know as the Barkhausen stability criterion. The gain and phase response of the closed-loop transfer function of the modulator at $f_{s} / 2$ can be expressed as:

$$
\begin{array}{r}
\left|G(s) \cdot H_{D A C}(s) \cdot H_{L}(s)\right|_{s=j \cdot \pi f_{s}}=1 \\
\left.\angle\left(G(s) \cdot H_{D A C}(s) \cdot H_{L}(s)\right)\right|_{s=j \cdot \pi f_{s}}=2 \pi,
\end{array}
$$

where $G$ and $H_{D A C}$ are the transfer functions of the quantizer and DAC, respectively. For example, a $1^{\text {st }}$ order $\Delta \Sigma \mathrm{M}$ is inherently stable for a bounded input signal and satisfies the gain and phase requirement defined by (2.8). The signal dependent gain of the quantizer guarantees a closed-loop gain of "1" [4]. 


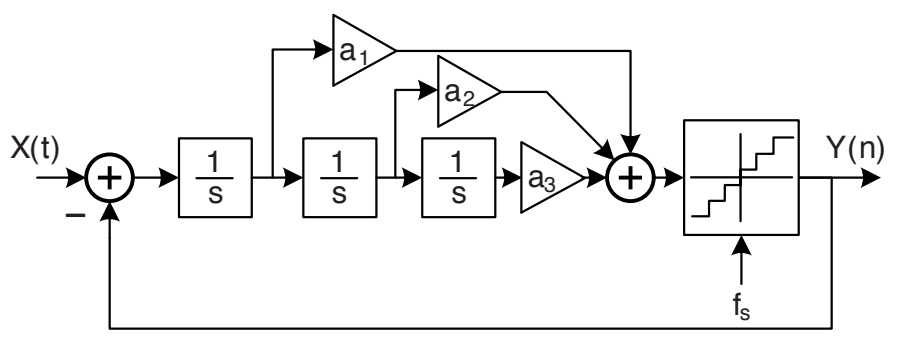

(a)

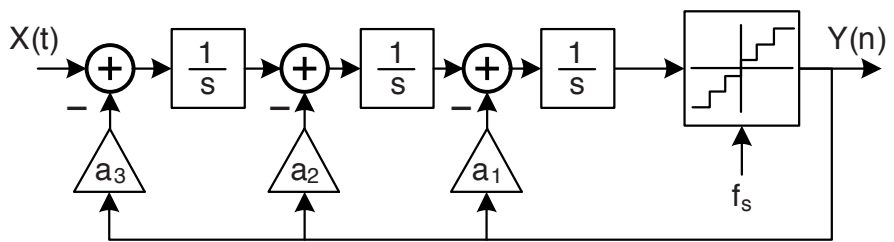

(b)

Figure 2.10 - A $3^{\text {rd }}$ order $\Delta \Sigma$ modulator with feedforward compensation (a), and with feedback compensation (b).

Moreover, the phase shift of the closed-loop is $360^{\circ}$, where the $1^{\text {st }}$ order loop filter, NRZ DAC (Section 2.1.3), and the sign inversion at the summation contribute $90^{\circ}, 90^{\circ}$, and $180^{\circ}$ of the phase shift, respectively.

For higher-order modulators, the phase shift of the loop filter increases to $(N \cdot \pi) / 2$. Therefore, a solution to $(2.8)$ does not exist and the modulator is unstable. To overcome this limitation, $(N-1)$ zeros are introduced to the transfer function, which can be expressed as:

$$
H_{L}(s)=\frac{\prod_{k=1}^{N-1}\left(s+s_{k}\right)}{s^{N}} .
$$

This can be achieved using a feedforward loop filter as shown in Fig. 2.10a. This loop filter architecture requires coefficients $\left(a_{1}, a_{2}, \ldots, a_{N}\right)$ and a summation node at the output of the loop filter. The STF of a modulator with a feedforward loop filter has an out-of-band peaking as shown in Fig. 2.11. Indeed, the modulator does amplify certain signals, which can be out-of-band blockers or interferers, therefore the system might require filtering before the modulator. On the other hand, the other STF shown in Fig. 2.11 does not exhibit any peaking. In this case, the loop filter employs the feedback architecture shown in Fig. 2.10b. However, the feedback loop filter requires $N \cdot D A C s$ to implement the coefficients $\left(a_{1}, a_{2}, \ldots, a_{N}\right)$, which increases the system complexity. 


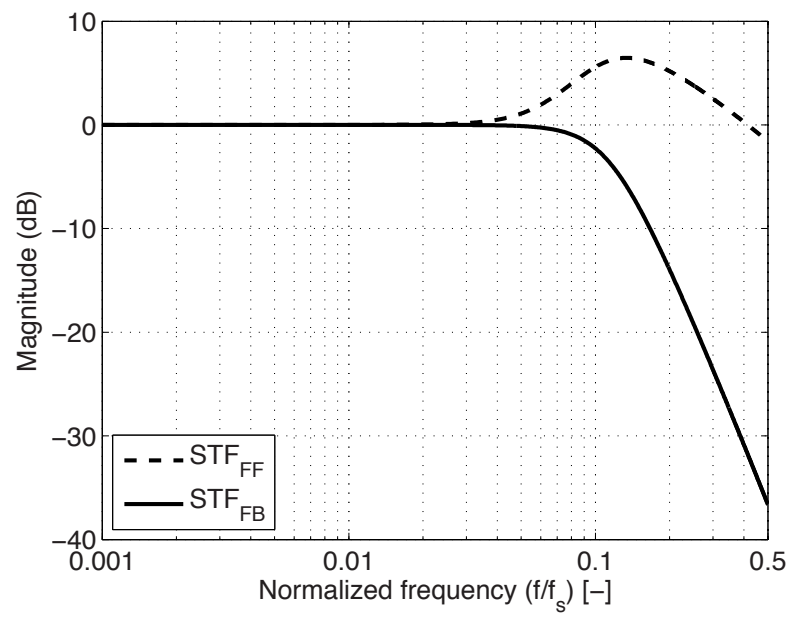

Figure 2.11 - Signal transfer function of a $3^{r d}$ order $\mathrm{CT} \Delta \Sigma$ modulator with a feedforward loop filter (dashed line) and a feedback loop filter (solid line).

The output of the modulator is fed back to the output of the each integrator stage. Therefore, the replica of the input signal is present at each integrator's output, which requires an amplifier that can generate a large output swing.

In practice, placing the loop filter zeros close to the poles reduces the effective gain of the loop filter so that $H_{L}(s)$ can be approximated as a $1^{\text {st }}$ order loop filter for frequencies around $0.5 \times f_{s}$. However, the signal-to-quantization noise ratio (SQNR) of the modulator is especially compromised for low oversampling ratios. In order to define a possible location of the zeros, the approach for Butterworth filters can be used in which the poles of filter is distributed evenly around the Left-Hand Plane (LHP) unit circle. Therefore, following (2.10), the zero locations can be expressed as:

$$
s_{k}=-\omega_{z} e^{\frac{j \pi}{2 n}(2 k+n-1)} \quad \text { where } \quad k=1,2,3, \ldots, N-1,
$$

where $\omega_{z}$ defines the location of the zero. By choosing a low enough $\omega_{z}$, a phase shift close to $90^{\circ}$ at $f_{s} / 2$ can be achieved without degrading the gain in the signal band too much. Figure 2.12 shows the bode plot of a $3^{\text {rd }}$ order feedforward loop filter which has Butterworth aligned zeros, and $\omega_{z}$ set to $0.025 \times f_{s}$, which results in a $96^{\circ}$ phase shift. However, this condition is not sufficient to guarantee a stable operation, therefore system-level simulations are still required to verify the stability of the modulator. 

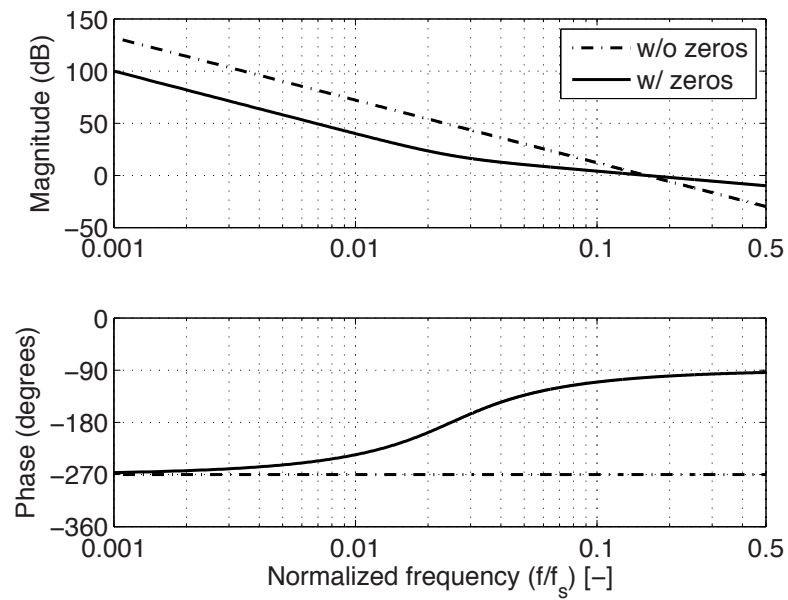

Figure 2.12 - Bode plot of the $3^{\text {rd }}$ order loop filter with Butterworth alignment of zeros (solid line) and without any zeros (dashed line).

\subsection{System-Level Non-Idealities}

This section discusses the system-level non-idealities in a $\Delta \Sigma \mathrm{M}$ such as: noise, nonlinearity, metastability and excess loop delay (ELD). Noise is an unwanted random fluctuation, which is common to all electronic circuits. Circuit noise limits the SNR. Nonlinearity is a behaviour of modulator's building block, in which the output signal does not follow the input in direct proportion. The nonlinearity of the blocks degrades the SFDR. ELD is the latency between the quantizer clock edge and the time when a change in the output of the DAC occurs [10-12]. The ELD can cause an unstable modulator, and in this case, the output of the modulator will not follow the input signal. Metastability exits in digital latches, in which the output of the latch persists at an unstable state for an unknown duration. The metastable state is not a valid digital state (i.e. "1", "0"), therefore introduces additional noise and reduces the SNR.

\subsubsection{Noise}

In a theoretical $\Delta \Sigma \mathrm{M}$, the quantization error fundamentally defines the maximum achievable SNR. To improve the SNR, the NTF of the modulator is optimized by carefully choosing system-level design parameters such as the order of the loop filter, the resolution of the quantizer, and the oversampling ratio 


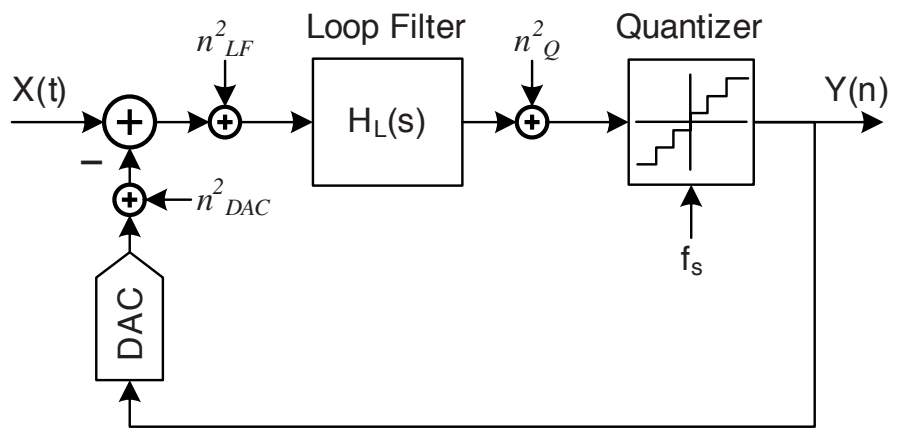

Figure 2.13 - Noise sources in a single-loop CT $\Delta \Sigma$ modulator.

(OSR). However, the building blocks of the modulator also introduce noise and degrade the SNR. Therefore, in an optimal ADC design (thermal noise limited), the quantization noise is set to at least $10 \mathrm{~dB}$ lower than the thermal noise.

The thermal noise of the building blocks sets a practical limit on the maximum achievable SNR $[13,14]$. The transfer function of the noise sources present in the modulator (Fig. 2.13) can be expressed as:

$$
Y^{2}=\left(n_{D A C}^{2}+n_{L F}^{2}\right) \cdot\left(\frac{H_{L}}{1+H_{L}}\right)^{2}+n_{Q}^{2} \cdot\left(\frac{1}{1+H_{L}}\right)^{2},
$$

where $n_{D A C}^{2}$ is the thermal noise of the DAC, $n_{L F}^{2}$ is the input referred thermal noise of the loop filter and $n_{Q}^{2}$ is the thermal noise of the quantizer referred to its input. The loop filter and the DAC are connected to the input of the ADC, therefore they are the most dominant noise sources. The loop filter mainly introduces thermal noise. In wide bandwidth modulators, the focus of this thesis, offset and $1 / f$ noise of the CMOS transistors can be neglected. Another unimportant noise source is the thermal noise of the quantizer $\left(n_{Q}^{2}\right)$ because it is also attenuated by the NTF. The decimation filter suppresses the noise that is outside of the signal bandwidth.

In addition to the thermal noise, the phase noise of the sampling clock decreases the SNR since the $\Delta \Sigma M$ is a sampled system. Due to the noisy sampling clock, the edges of the DAC output are not well-defined. This effect can be quantified by the signal-to-jitter-noise-ratio (SJNR), which is the ratio of the signal power to the jitter noise power at the output of the modulator.

In most cases, the clock of an ADC is specified in terms of root-meansquare (RMS) jitter rather than in terms of phase noise as is commonly done 


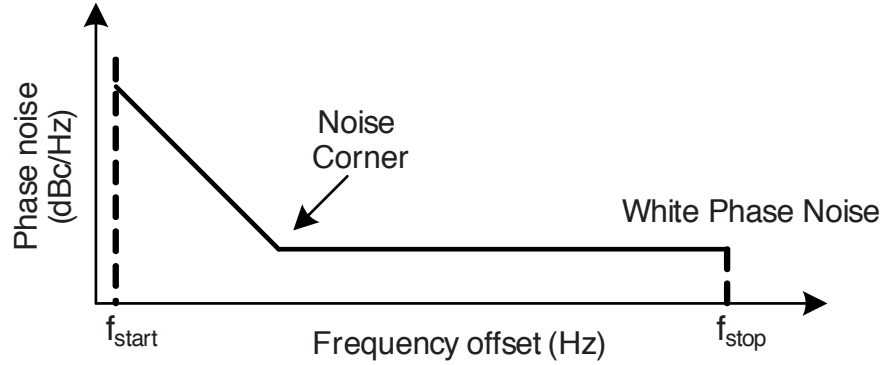

(a)

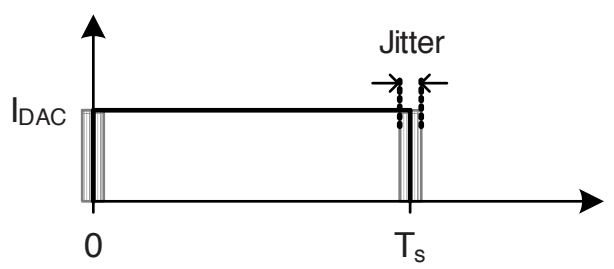

(b)

Figure 2.14 - The phase noise of an oscillator (a), and the effect of clock jitter on the DAC pulse shape (b).

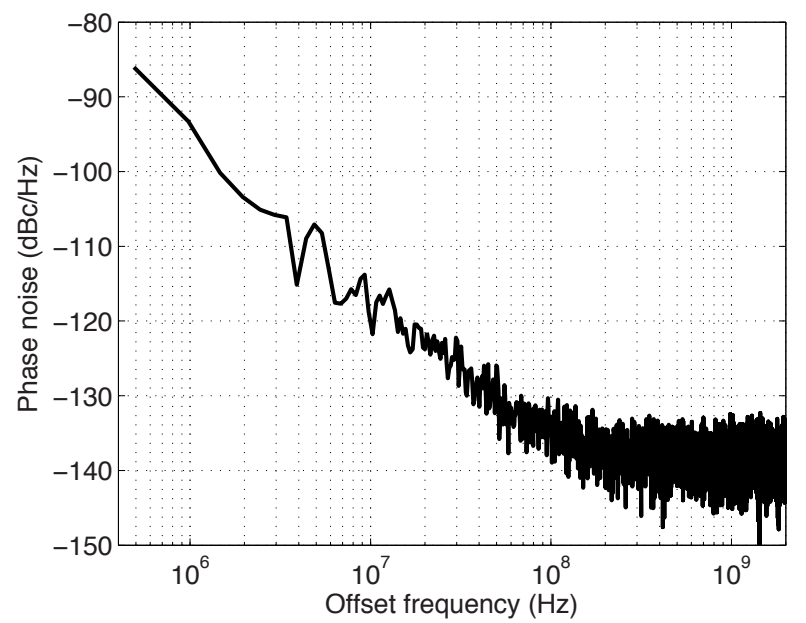

Figure 2.15 - The single side-band spectrum of a non-ideal sampling clock. 


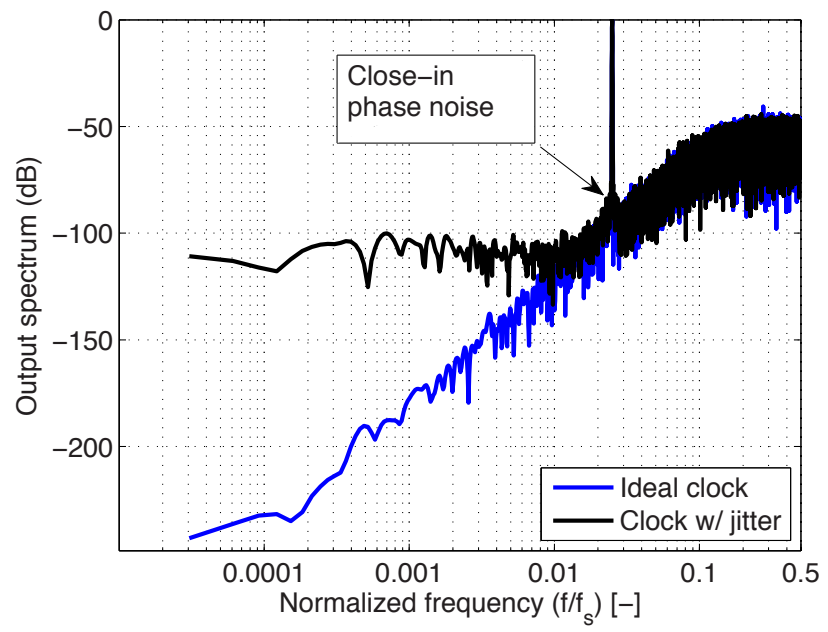

Figure 2.16 - The output spectrum of the $3^{\text {rd }}$ order CT $\Delta \Sigma$ modulator with a non-ideal sampling clock. (FFT size is $2^{15}$ pts.)

in oscillators or clock sources. Figure 2.14a illustrates the phase noise of an oscillator, from which the jitter specifications can be derived. The phase noise increases for frequencies less than the noise corner. For frequencies beyond the noise corner, the oscillator noise spectrum is white, and is determined by the noise of the output buffers of the oscillator. The RMS jitter can be estimated as [15]:

$$
\operatorname{Jitter}(R M S)=\frac{\sqrt{2 \cdot 10^{I P N / 10}}}{2 \pi \cdot f_{c l k}},
$$

where IPN is the integrated phase noise from $f_{\text {start }}$ to $f_{\text {stop }}$. The $f_{\text {start }}$ depends on the spectral resolution required by the application. In practice, $f_{\text {start }}$ as low as $10-100 \mathrm{~Hz}$ is common and $f_{\text {stop }}$ is set to the sampling frequency of the ADC assuming that the bandwidth of the clock input is limited to the sampling frequency. For a $\Delta \Sigma \mathrm{M}, f_{\text {stop }}$ is set to the oversampled clock frequency.

The noise due to the clock jitter depends both on the implementation of the feedback DAC and the clock source. If we assume that the DAC is implemented with NRZ pulses, the phase noise will distort the DAC pulse shape (Fig. 2.14b). An NRZ DAC is advantageous because it only switches when the data toggles. Therefore, it introduces less noise compared to an RZ DAC [16].

Since the DAC is connected to the input of the ADC, the clock jitter-induced errors also appear at the output of the ADC without any filtering. For a $\Delta \Sigma \mathrm{M}$ 
aiming at $\mathrm{GHz}$ sampling frequencies, the effect of phase noise can limit the SNR. The phase noise of the clock convolves with the input signal, and the ADC's selectivity will be limited by the close-in phase noise of the oscillator. On the other hand, the white noise of the oscillator mixes with the quantization noise and down-converts it into the baseband. This increases the in-band noise and thus limits the dynamic range of the ADC [17].

At the system level, the effect of clock jitter can be simulated in two steps. First of all, a square-wave clock signal is generated based on the phase noise model of a clock source in MATLAB. The phase noise spectrum of the clock source is shown in Fig. 2.15. Then the behaviorial model of a $3^{\text {rd }}$ order $\Delta \Sigma \mathrm{M}$ with a 4-bit quantizer is simulated in Simulink. The multi-bit DAC of the modulator is triggered with the clock source generated in MATLAB; the effect of clock jitter is shown in Fig. 2.16. As explained before, the close-in phase noise of the clock can be observed around the input signal, and the white-noise of the clock increases the in-band noise floor.

\subsubsection{Non-Linearity}

As explained in Section 2.1.2, the quantizer is the only inherently non-linear building block of the modulator. A single-bit quantizer demonstrates the highest non-linearity, although when placed in a $\Delta \Sigma \mathrm{M}$, the non-linearity of the quantizer is suppressed by the gain of the loop filter. Fig. 2.17 shows an FFT of the simulated output of a $3^{\text {rd }}$ order single-bit $\Delta \Sigma$ ADC with a full scale input signal. Especially, $\mathrm{HD}_{3}$ is present at the output of the modulator. To further reduce and de-correlate $\mathrm{HD}_{3}$, additional dithering can be applied to the input of the quantizer [4], however, reducing maximum stable input amplitude of the modulator.

A multi-bit quantizer is intrinsically more linear than a single-bit comparator. A $\Delta \Sigma \mathrm{M}$ with a multi-bit quantizer does not generate visible harmonic distortion (HD) tones and can also achieve more aggressive noise shaping. Such multi-bit modulators usually employ multi-bit DACs. In a practical implementation, each DAC unit will deviate from its nominal value due to the mismatch introduced by the process variation, so the multi-bit DAC introduces distortion. The standard deviation of a DAC unit is usually in the order of $0.1 \%-10 \%$ in the current fabrication processes. Fig. 2.18 shows an FFT of the simulated output of a 4 -bit $3^{r d}$ order $\Delta \Sigma \mathrm{ADC}$ with $\sigma_{I_{D A C}} / I_{D A C}=0.2 \%$. It can be seen that DAC mismatch limits the linearity of a multi-bit $\Delta \Sigma \mathrm{M}$. However, this limitation can be overcome by various techniques such as: dynamic element matching (DEM) and calibration of DAC current sources [18-21], but these techniques increase the complexity of the system. 


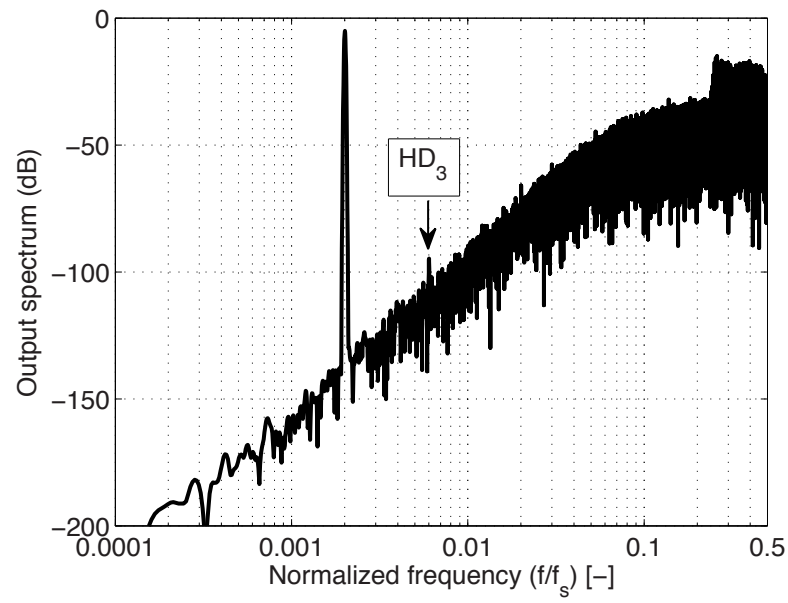

Figure 2.17 - The harmonic tones due to the inherent non-linearity of a singlebit quantizer. (FFT size is $2^{17}$ pts.)

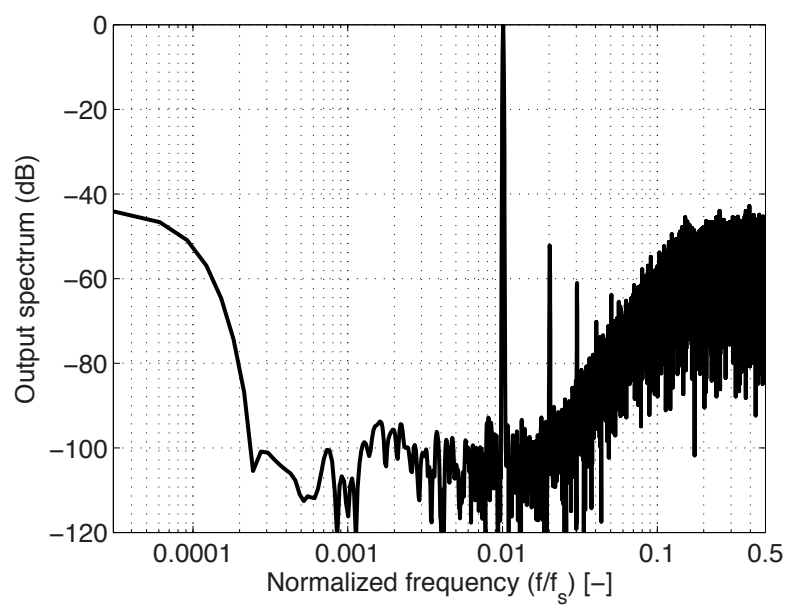

Figure 2.18 - The harmonic tones due to the mismatch of a multi-bit DAC. (FFT size is $2^{17}$ pts.) 


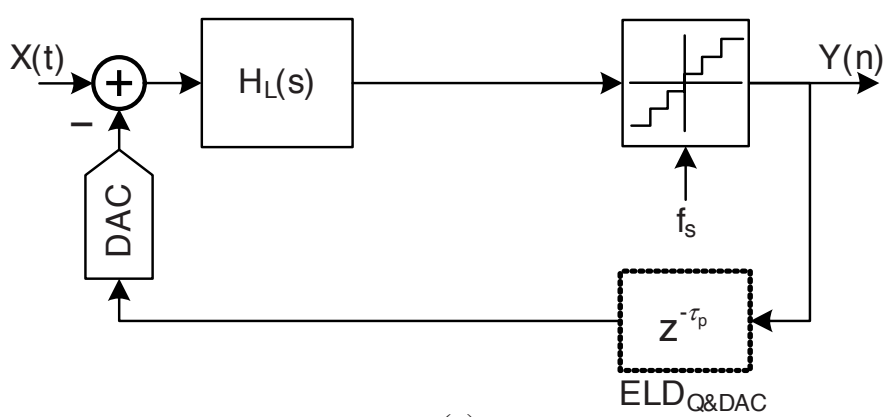

(a)

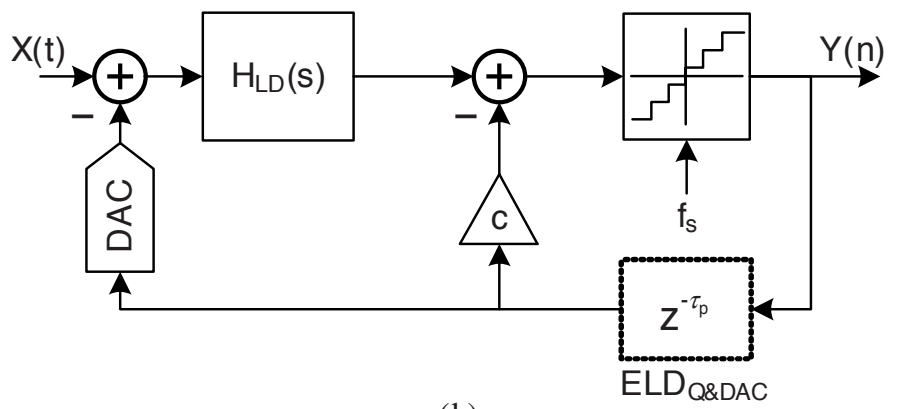

(b)

Figure 2.19 - Excess loop delay (ELD) in a single-loop CT $\Delta \Sigma$ modulator (a), and the accompanying ELD compensation technique (b).

\subsubsection{Excess Loop Delay}

As explained in the previous section, the stability of a $\Delta \Sigma \mathrm{M}$ relies on the amplitude and phase response of the loop. However, in a real implementation, the building blocks also introduce ELD, which is defined as the time delay between the quantizer clock edge and the time when a change in the output of the DAC occurs [10-12]. ELD is basically caused by the limited speed of the transistors used to implement the quantizer and the DAC of a $\Delta \Sigma \mathrm{M}$. As shown in Fig. 2.19a, it can be modeled as a discrete time delay $z^{-\tau_{p}}$. As the ELD increases, the phase shift in the loop increases, which ultimately causes the $\Delta \Sigma \mathrm{M}$ to become unstable.

To illustrate the effect of ELD, the amplitude and phase response of the loop filter of a $3^{r d}$ order 4 -bit $\Delta \Sigma \mathrm{M}$ with a one-clock period of ELD is shown in Fig. 2.20. The amplitude and phase response of the DAC and the summation node 

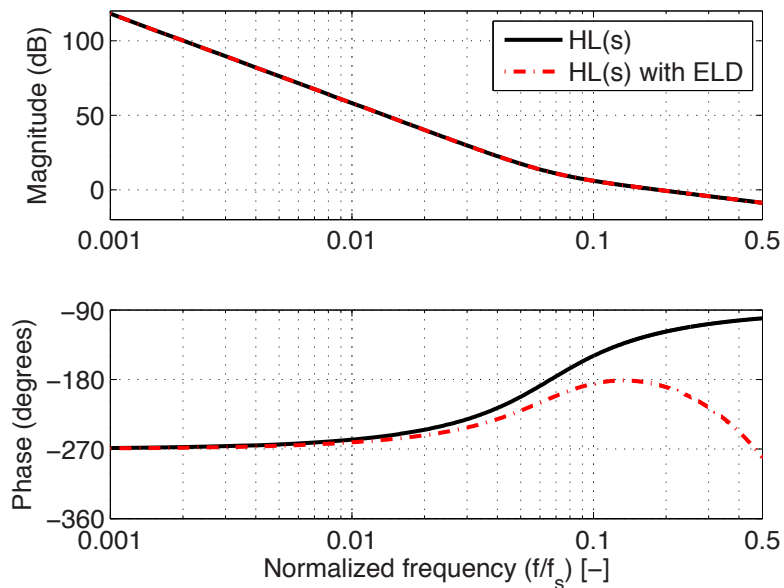

Figure 2.20 - Amplitude and phase response of the loop filter with and without excess loop delay.

at the input of the modulator have been neglected. The amplitude response of the loop filter is not affected, but the phase response of the loop filter (designed to achieve a phase shift of $90^{\circ}$ ) is degraded due to the ELD. From our previous analysis, we can conclude that a modulator with a one-clock cycle delay is unstable. The exact relation between the stability and the ELD depends on the design of the modulator.

As shown in Fig. 2.21, the SQNR of the modulator stays flat up to $0.3 \times T_{s}$ ELD. However, the modulator is not stable beyond this value. An in-depth study of the simulation results reveals that non-zero ELD causes the output swing of the integrators to increase beyond their designed values. Furthermore, any clipping in a practical implementation, which is especially a problem at the summation node, can push the modulator into instability for much smaller values of ELD.

To compensate the increase in phase shift due to ELD and recover from an unstable mode of operation, the modulator requires an additional zero that will bypass the loop filter at $f_{s} / 2$. This is achieved by introducing a feedback DAC with a coefficient $(c)$ around the quantizer as shown in Fig. 2.19b [11,22]. Since the calculation of the loop-filter coefficients is straightforward in the $\mathcal{Z}$ domain, the continuous-time loop filter $\left(H_{L}(s)\right)$ is transformed to its discretetime equivalent $\left(H_{L, d t}(z)\right)$ by using the impulse-invariant transformation [1], which can also be expressed as: 


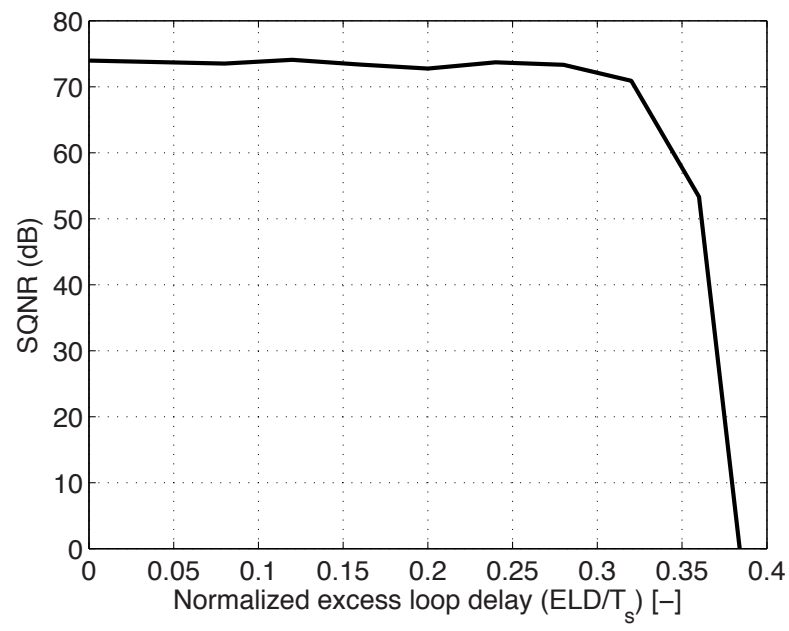

Figure 2.21 - The SQNR of the $3^{\text {rd }}$ order CT $\Delta \Sigma$ modulator with excess loop delay.

$$
H_{L, d t}(z)=\mathcal{Z}\left\{\left.\mathcal{L}^{-1}\left\{H_{D A C}(s) \cdot H_{L}(s)\right\}\right|_{t=n T_{s}}\right\} .
$$

In order to find the discreet-time (DT) equivalent of the continuous-time (CT) loop filter, the impulse-invariant transformation is preferred since we assume that two modulators are equivalent, for a given input signal, if their loop filter generates the same outputs at the sampling moments of the their quantizers [23]. Mapping of a CT loop filter to a DT equivalent is only valid for $f \ll f_{s}$. However, for the following analysis (2.16-2.18), we rely on (2.13) which maps the sampled intances of the CT loop filter into its discrete-time equivalent.

In general, the main motivation of the ELD compensation technique is to preserve the original NTF of the modulator and thus the stability of the modulator. Therefore, a new loop filter $\left(H_{L D, d t}(z)\right)$ is required to keep the same NTF. So from the viewpoint of stability, the new loop filter $\left(H_{L D, d t}(z)\right)$ can be determined from:

$$
H_{L D, d t}(z)=H_{L, d t}(z) z^{\tau_{p}}-c,
$$

where the feedback DAC has an NRZ waveform. The continuous-time equivalent of the new loop filter is then calculated by applying the inverse of the impulse-invariant transformation (2.13). 
Assuming both the $H_{L, d t}(z)$ and $H_{L D, d t}(z)$ are implemented by using the same filter order, ELD up to one clock cycle delay can be compensated by using (2.14) and the modulator achieves the same SQNR and NTF. For the ELD more than one clock cycle, a solution to (2.14) does not exist since the $H_{L, d t}(z)$ and $H_{L D, d t}(z)$ have the same filter architecture. A $\Delta \Sigma \mathrm{M}$ which uses the ELD compensation technique shown in Fig. 2.19b is unstable for ELD more than one clock cycle.

For example, a $2^{\text {nd }}$ order modulator with an ideal $\operatorname{NTF}(z)=\left(1-z^{-1}\right)^{2}$ has a discrete-time equivalent loop filter which is:

$$
\begin{aligned}
H_{L, d t}(z) & =\frac{1-N T F(z)}{N T F(z)} \\
\frac{a_{1} z^{-1}+a_{2} z^{-2}}{1-2 z^{-1}+z^{-2}} & =\frac{2 z^{-1}-z^{-2}}{1-2 z^{-1}+z^{-2}} \\
a_{1} & =2 \\
a_{2} & =-1
\end{aligned}
$$

The continuous-time equivalent of the loop filter with a NRZ DAC pulse can be determined by inverting (2.13):

$$
H_{L}(s)=\frac{1.5}{s}+\frac{1}{s^{2}} .
$$

Assuming there is one clock cycle delay $\left(z^{\tau_{p}}=z^{1}\right)$, the new loop filter $\left(H_{L D, d t}(z)\right)$ will have the same structure as the original loop filter and following (2.14):

$$
\begin{aligned}
H_{L D, d t}(z) & =H_{L, d t}(z) \cdot z^{1}-c \\
\frac{a_{1 d} z^{-1}-a_{2 d} z^{-2}}{1-2 z^{-1}+z^{-2}} & =\frac{a_{1} z^{-1}-a_{2} z^{-2}}{1-2 z^{-1}+z^{-2}} \cdot z^{1}-c \\
a_{1 d} & =2 a_{1}+a_{2}=3 \\
a_{2 d} & =a_{1}=2 \\
c & =a_{1}=2 \\
H_{L D, d t}(z) & =\frac{3 z^{-1}-2 z^{-2}}{1-2 z^{-1}+z^{-2}} .
\end{aligned}
$$

The continuous-time equivalent of the new loop filter with a NRZ DAC pulse can be determined by inverting (2.13):

$$
H_{L D}(s)=\frac{2.5}{s}+\frac{1}{s^{2}} .
$$




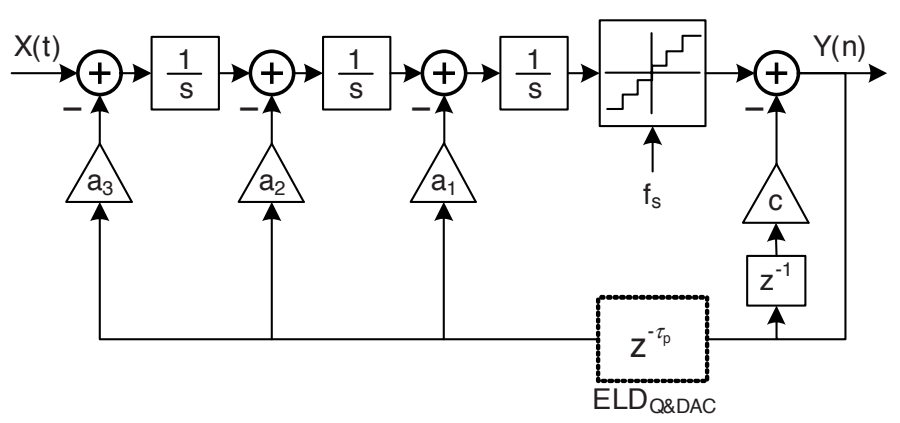

Figure 2.22 - The digital ELD compensation technique.

Even though the modulator has the same NTF, the STF of the modulator is modified since there exists a new loop filter $\left(H_{L D}(s)\right)$. As a result, the new STF of the modulator is expressed as:

$$
\left.\operatorname{STF}_{D}(s)\right|_{s=j \omega}=\left.\left.H_{L D}(s)\right|_{s=j \omega} \cdot \operatorname{NTF}(z)\right|_{z=e^{j \omega T_{s}}} .
$$

In particular, the peaking in the STF of the $\Delta \Sigma \mathrm{M}$ increases and the center frequency of the peaking shifts to a higher frequency. This will be explained in more detail in Section 3.1.3.

In addition to the ELD compensation technique shown in Fig. 2.19b, an attractive solution that can be implemented in CMOS processes is to compensate for the loop delay in the digital domain as shown in Fig. 2.22 [24]. However, extra hardware is required which introduces additional delay and further pushes the digital circuitry to its limits. A part of the dynamic range (DR) is used for compensating the delay in the digital domain [25]. Considering those drawbacks, an analog delay compensation method is preferred in designs which aim for a high sampling speed.

To maintain the NTF and satisfy the stability requirements of the modulator, the summation node presented in Fig. 2.19b should not introduce additional ELD. A summation node can be implemented in analog domain by the use of active amplifiers. An interesting modification to the analog ELD compensation is to place the summation node at the input of the last integrator. A possible implementation of this technique is shown in Fig. 2.23. By using this technique, the additional summation node that is required for the ELD compensation is not necessary anymore. However, the input to the coefficient (c) must be differentiated in the digital domain $\left(1-z^{-0.5}\right)$ to implement a summation node [26]. To preserve stability, the amplifier that implements the last integrator must have a wide bandwidth for a minimal delay [25], as well 


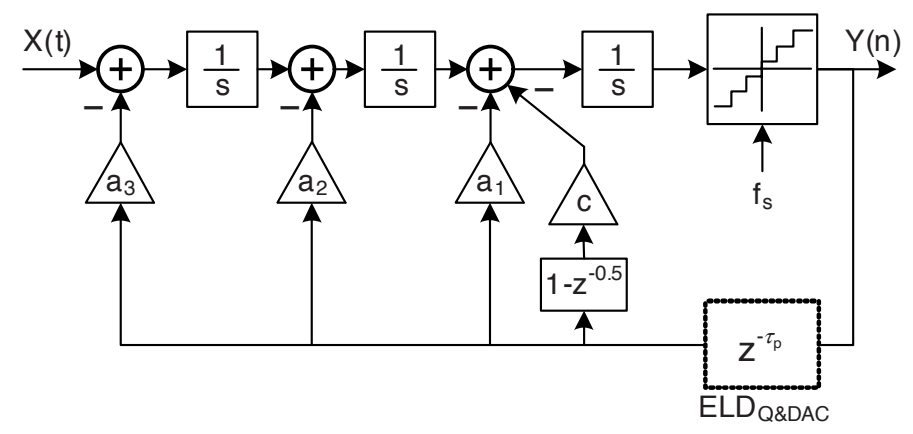

Figure 2.23 - The ELD compensation technique which uses the summation node of the last integrator.

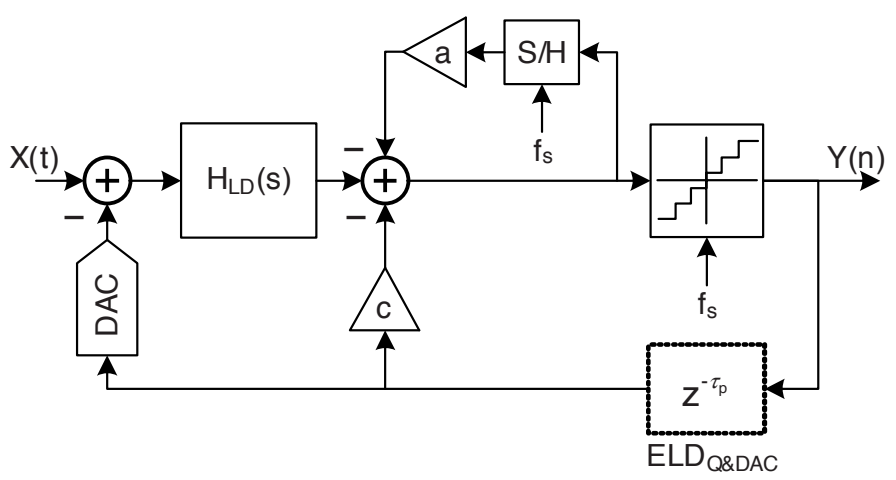

Figure 2.24 - The ELD compensation technique which bypasses the quantizer with an auxiliary fast loop. 


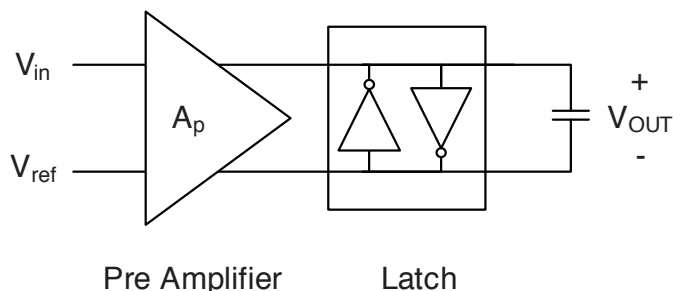

Figure 2.25 - Block diagram of the comparator.

as high gain for reducing the variation of the loop-filter coefficients over process, voltage, and temperature (PVT). These stringent requirements result in a power-hungry summing amplifier.

The ELD compensation techniques described above can compensate for up to a one-clock period delay without losing any SQNR. In the case of larger ELD, the maximum input amplitude of the modulator will decrease, which will result in a loss in DR and eventually cause the modulator to become unstable. To overcome this limitation, the quantizer can be bypassed by an auxiliary fast loop which is implemented by a sample-and-hold $(\mathrm{S} \& \mathrm{H})$ and a scaling coefficient $(c)$ [27] shown in Fig. 2.24. The auxiliary fast loop measures the output of the loop filter and compensates the phase shift due to more than one-clock period of ELD. This approach can compensate for $1.5 T_{s}$ of ELD at the cost of reducing the order of noise shaping by one [27].

\subsubsection{Metastability}

To achieve very high sampling rates, a flash ADC is often employed as the quantizer of a $\Delta \Sigma \mathrm{M}$. An N-bit flash ADC employs $2^{N}$ comparators. Each comparator employs a digital latch which suffers from metastability errors for very small input signals $[28,29]$. As a result, the latches make wrong decisions and the digital output code of the flash ADC will have errors. Multi-bit flash ADCs are especially prone to metastability since the input signal for each comparator decreases as the resolution of the flash ADC increases.

High-speed flash ADCs usually employ pipeline stages to reduce metastability errors; however, this increases their latency. As explained in Section 2.2.3, the additional delay of the quantizer causes instability. Therefore, the output of a flash $\mathrm{ADC}$ in a $\triangle \Sigma \mathrm{M}$ is directly connected to the following stages such as the feedback DAC, which requires a co-design of the quantizer and the DAC. 


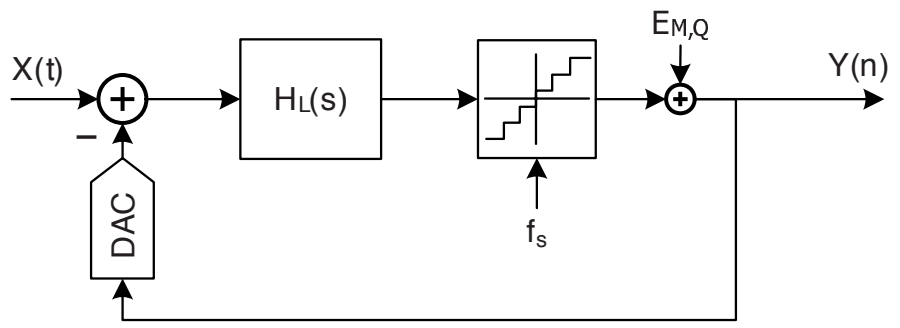

(a)

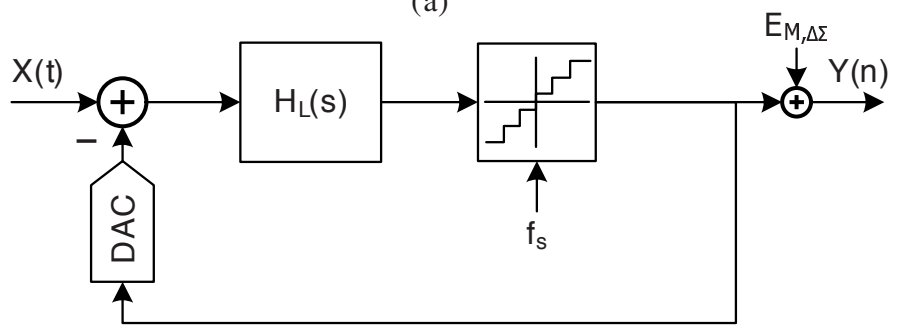

(b)

Figure 2.26 - A basic single-loop continuous-time $\Delta \Sigma$ modulator with BER. Bit errors are introduced at the output of the quantizer (a) and the output of the DAC (b).

Furthermore, the performance of the $\Delta \Sigma \mathrm{M}$ must be simulated in the presence of metastability errors.

Metastable states of a comparator are usually very difficult to observe. Instead, the bit-error-ratio (BER), which is defined as the number of metastable states of a comparator per second, gives more insight at the system level. Assuming that a comparator has a pre-amplifier and a latch as shown in Fig. 2.25, the comparator's BER can be shown to be given by [30]:

$$
B E R=\frac{0.5 V_{\text {logic }}}{V_{F S} A_{\text {pre }}} \cdot e^{-\frac{A_{0}-1}{\tau} t_{d}},
$$

where $V_{\text {logic }}$ is the output voltage level, $V_{F S}$ is the full-scale input range of the comparator, $A_{\text {pre }}$ is the gain of the pre-amplifier of the comparator, $A_{0}$ is the gain of the regenerative latch, $\tau$ is the time constant of the latch, and $t_{d}$ is the operation time of the comparator. In most cases, the comparator is only used during half of a clock period, so $t_{d}$ is set to $T_{s} / 2$. The metastability errors of the quantizer are shaped by the gain of the loop filter. However, the feedback DAC connected to the input of the modulator often uses a D-FF to 


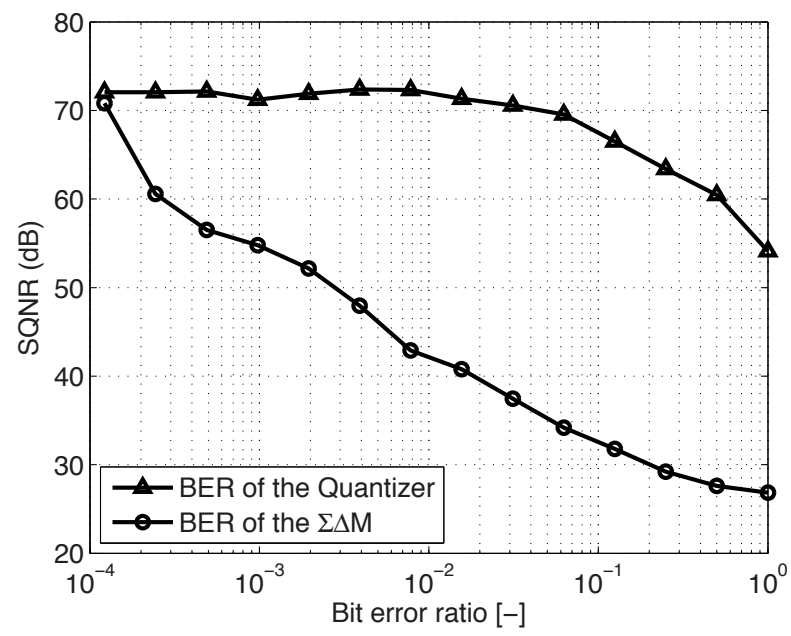

Figure 2.27 - Effect of the BER in a $3^{\text {rd }}$ order CT $\Delta \Sigma$ modulator. Fig. 2.26a models the BER of the Quantizer. Fig. 2.26b models the BER of the $\Delta \Sigma \mathrm{M}$. (For each simulation, FFT size is $2^{14}$ pts.)

re-time the data signal of the quantizer and enable distribution of a low-jitter clock signal. The metastability errors introduced by this D-FF at the output of the DAC, which are present at the input of the modulator, degrade the performance dramatically. In this thesis, the bit errors introduced by the D$\mathrm{FF}$ of the feedback DAC are considered as the bit errors of the modulator. In other words, bit-errors of the modulator occur when the output of the DAC which drives the DAC current sources differs from the digital output of the modulator.

Figure 2.26a and 2.26b models the BER of the quantizer and the modulator, respectively. For each case, bit errors are introduced during the simulation with an amplitude of $1 L S B$ and distributed randomly through out the simulation time. The simulation models the practical operation of the modulator, since only one slice of the comparator has a critical input voltage $\left(V_{\text {in }}<V_{\text {tap }}\right)$ and the input voltage of the other comparators are larger than $\left(V_{i n}>V_{t a p}\right)$, which forces them to give a correct decision. The DAC unit connected to the critical comparator has the highest chance of introducing the bit errors.

Figure 2.27 shows the SNR of a 4 -bit $3^{r d}$ order $\Delta \Sigma \mathrm{M}$ in the presence of bit errors. The input signal is set to full scale and the SNR stays fairly constant for $\mathrm{BER}<O S R^{-1}$ because the bit errors act as a white noise source at the 
output of the quantizer and are shaped by the NTF. However, we should note that as the BER increases, the output voltage of the integrators increases. In a practical implementation, the SNR can degrade further if the integrators of the modulator saturate. On the other hand, as shown in Fig. 2.27, the BER of the modulator degrades the SNR dramatically, because the meta-stability errors are not shaped by the modulator's NTF. Therefore, the feedback path of the modulator must have enough gain to adequately suppress the BER below the aimed noise level.

Furthermore, a $\Delta \Sigma \mathrm{M}$ is often followed by digital blocks such as a thermometerto-binary decoder or a decimation filter, which use latches, and are also subject to meta-stability. Therefore, any error introduced in the digital back-end will also degrade the modulator's SQNR.

\subsection{Summary}

This chapter has presented the operation of an ideal single-loop CT $\Delta \Sigma$ modulator and described its main building blocks. The quantizer, which converts the signals into digital, is the only non-linear block of the modulator and has a phase uncertainty which is quite significant in the case of a single-bit quantizer. The non-linear behavior of the quantizer has significant effect on the modulator. Furthermore, the single-bit quantizer creates harmonic distortion and intermodulation tones. It has been shown that for a sine-wave input, the harmonic distortion and intermodulation product of a quantizer can be modeled accurately, and the presence of white noise at the input of the quantizer improves the harmonic distortion and intermodulation product at the cost of a reduced SNR.

Many types of DAC output waveforms can be implemented in a $\Delta \Sigma \mathrm{M}$, but due to the focus on $\mathrm{GHz}$ sampling frequencies in this thesis, only NRZ and RZ DAC types have been analyzed. The DAC introduces a $\mathrm{ZOH}$ function in the feedback and its amplitude and phase response is defined by the shape of the DAC output waveform.

A $\Delta \Sigma \mathrm{M}$ with a $1^{\text {st }}$ order loop filter is inherently stable because the loop filter has a $90^{\circ}$ phase shift. To design a stable modulator with a higher order loop filter, the phase shift of the loop filter must be close to $90^{\circ}$ at $f_{s} / 2$. A complete analysis of its stability is complicated by the fact that the quantizer is a non-linear element. In most practical cases, the stability of a $\Delta \Sigma \mathrm{M}$ is verified by computer simulations.

System level non-idealities such as noise, linearity, metastability and excess loop delay (ELD) limit the performance of the modulator. The DAC and the 
first stage of the loop filter are the most dominant sources of noise because they are directly connected to the input of the modulator. Furthermore, the mismatch of a multi-bit DAC also degrades linearity. The metastability of the quantizer can be modeled as white noise added to the output of the quantizer, which then degrades SNR. If the ELD of the quantizer is too much, it will result in an unstable modulator. All the non-idealities have been analyzed by system-level simulations. In the next chapter, the system-level and detailed block-level requirements of a CT $\Delta \Sigma$ modulator which can achieve a $125 \mathrm{MHz}$ signal bandwidth with a 70dB DR will be derived.

\section{References}

[1] F. Gardner, "A transformation for digital simulation of analog filters," in Communications, IEEE Transactions on, vol. 34, no. 7, pp. 676-680, July 1986.

[2] O. Shoaei, Continuous-Time Delta-Sigma A/D Converters for High Speed Applications. Ph.D. dissertation, Carleton University, 1995.

[3] M. Ortmanns and F. Gerfers, Continuous-Time Sigma-Delta A/D Conversion, Fundamentals, Error Correction and Robust Implementations. Springer, 2005.

[4] S. Norsworthy, R. Schreier, and G. Temes, Delta-Sigma Data Converters (Theory, Design, and Simulation). John Wiley and Sons, Inc, 1996.

[5] R. Schreier and G. Temes, Understanding Delta-Sigma Data Converters. John Wiley and Sons, Inc, 2004.

[6] M. Hofelt, "On the stability of a 1-bit-quantized feedback system," in Acoustics, Speech, and Signal Processing, IEEE International Conference on ICASSP 'r9., vol. 4, Apr. 1979, pp. 844-848.

[7] J. v. Engelen and R. v. d. Plassche, Bandpass Sigma Delta Modulators Stability Analysis, Performance and Design Aspects. Springer, 2000.

[8] N. Blachman, "The intermodulation and distortion due to quantization of sinusoids," in Acoustics, Speech and Signal Processing, IEEE Transactions on, vol. 33, no. 6, pp. 1417-1426, Dec. 1985.

[9] S. Ardalan and J. Paulos, "An analysis of nonlinear behavior in delta-sigma modulators," in Circuits and Systems, IEEE Transactions on, vol. 34, no. 6, pp. 593-603, June 1987. 
[10] J. A. Cherry and W. M. Snelgrove, Continuous-Time Delta-Sigma Modulators for High-Speed A/D Conversion: Theory, Practice and Fundamental Performance Limits. Norwell, MA, USA: Kluwer Academic Publishers, 2000.

[11] P. Benabes, M. Keramat, and R. Kielbasa, "A methodology for designing continuous-time sigma-delta modulators," in European Design and Test Conference, 199\%., Mar. 1997, pp. 46-50.

[12] O. Shoaei, "Continuous-time delta-sigma A/D converters for high speed applications," Ph.D. dissertation, Carleton Univ., Ottawa, ON, 1996.

[13] B. Murmann, "A/D converter trends: Power dissipation, scaling and digitally assisted architectures," in Custom Integrated Circuits Conference, 2008. CICC 2008. IEEE, Sept. 2008, pp. 105-112.

[14] E. Vittoz, "The design of high-performance analog circuits on digital CMOS chips," in Solid-State Circuits, IEEE Journal of, vol. 20, no. 3, pp. 657-665, June 1985.

[15] B. Brannon, Sampled systems and the effects of clock phase noise and jitter application note, Analog Devices, Inc., USA. [Online]. Available: http://www . analog.com

[16] E. van der Zwan and E. Dijkmans, "A 0.2-mW CMOS $\Sigma \Delta$ modulator for speech coding with $80 \mathrm{~dB}$ dynamic range," in Solid-State Circuits, IEEE Journal of, vol. 31, no. 12, pp. 1873-1880, Dec. 1996.

[17] R. van Veldhoven, P. Nuijten, and P. van Zeijl, "The effect of clock jitter on the DR of $\Sigma \Delta$ modulators," in Circuits and Systems, 2006. Proceedings. 2006 IEEE International Symposium on, May 2006, p. 4.

[18] R. Baird and T. Fiez, "Improved $\Delta \Sigma$ DAC linearity using data weighted averaging," in Circuits and Systems, 1995. ISCAS '95., 1995 IEEE International Symposium on, vol. 1, Apr. 1995, pp. 13-16 vol.1.

[19] M. Tiilikainen, "A 14-bit 1.8V 20mW 1 $\mathrm{mm}^{2}$ CMOS DAC," in Solid-State Circuits, IEEE Journal of, vol. 36, no. 7, pp. 1144-1147, jul 2001.

[20] R. Schreier and B. Zhang, "Noise-shaped multibit d/a convertor employing unit elements," in Electronics Letters, vol. 31, no. 20, pp. 1712-1713, Sept. 1995. 
[21] J. Silva et al., "Digital techniques for improved $\Delta \Sigma$ data conversion," in Custom Integrated Circuits Conference, 2002. Proceedings of the IEEE 2002, 2002, pp. 183-190.

[22] S. Yan and E. Sanchez-Sinencio, "A continuous-time sigma-delta modulator with 88-dB dynamic range and 1.1-MHz signal bandwidth," in SolidState Circuits, IEEE Journal of, vol. 39, no. 1, pp. 75-86, Jan. 2004.

[23] J. A. Cherry and W. M. Snelgrove, Continuous-Time Delta-Sigma Modulators for High-Speed A/D Conversion: Theory, Practice and Fundamental Performance Limits. Norwell, MA, USA: Kluwer Academic Publishers, 2000.

[24] P. Fontaine, A. Mohieldin, and A. Bellaouar, "A low-noise low-voltage CT delta-sigma modulator with digital compensation of excess loop delay," in Solid-State Circuits Conference, 2005. Digest of Technical Papers. ISSCC. 2005 IEEE International, Feb. 2005, pp. 498-613 Vol. 1.

[25] M. Keller et al., "A comparative study on excess-loop-delay compensation techniques for continuous-time sigma-delta modulators," in Circuits and Systems I: Regular Papers, IEEE Transactions on, vol. 55, no. 11, pp. 3480-3487, Dec. 2008.

[26] G. Mitteregger et al., "A 20-mW 640-MHz CMOS continuous-time $\Sigma \Delta$ ADC with 20-MHz signal bandwidth, 80-dB dynamic range and 12-bit ENOB," in Solid-State Circuits, IEEE Journal of, vol. 41, no. 12, pp. 2641-2649, Dec. 2006.

[27] V. Singh et al., "A 16 MHz BW 75 dB DR CT ADC compensated for more than one cycle excess loop delay," in Solid-State Circuits, IEEE Journal of, vol. 47, no. 8, pp. 1884-1895, Aug. 2012.

[28] H. Veendrick, "The behaviour of flip-flops used as synchronizers and prediction of their failure rate," in Solid-State Circuits, IEEE Journal of, vol. 15 , no. 2, pp. 169-176, apr 1980.

[29] J. Cherry and W. Snelgrove, "Clock jitter and quantizer metastability in continuous-time delta-sigma modulators," in Circuits and Systems II: Analog and Digital Signal Processing, IEEE Transactions on, vol. 46, no. 6, pp. 661-676, June 1999.

[30] R. van de Plassche, CMOS Integrated Analog-to-Digital and Digital-toAnalog Converters. Dordrecht, the Netherlands: Kluwer Academic Publishers, 2003. 


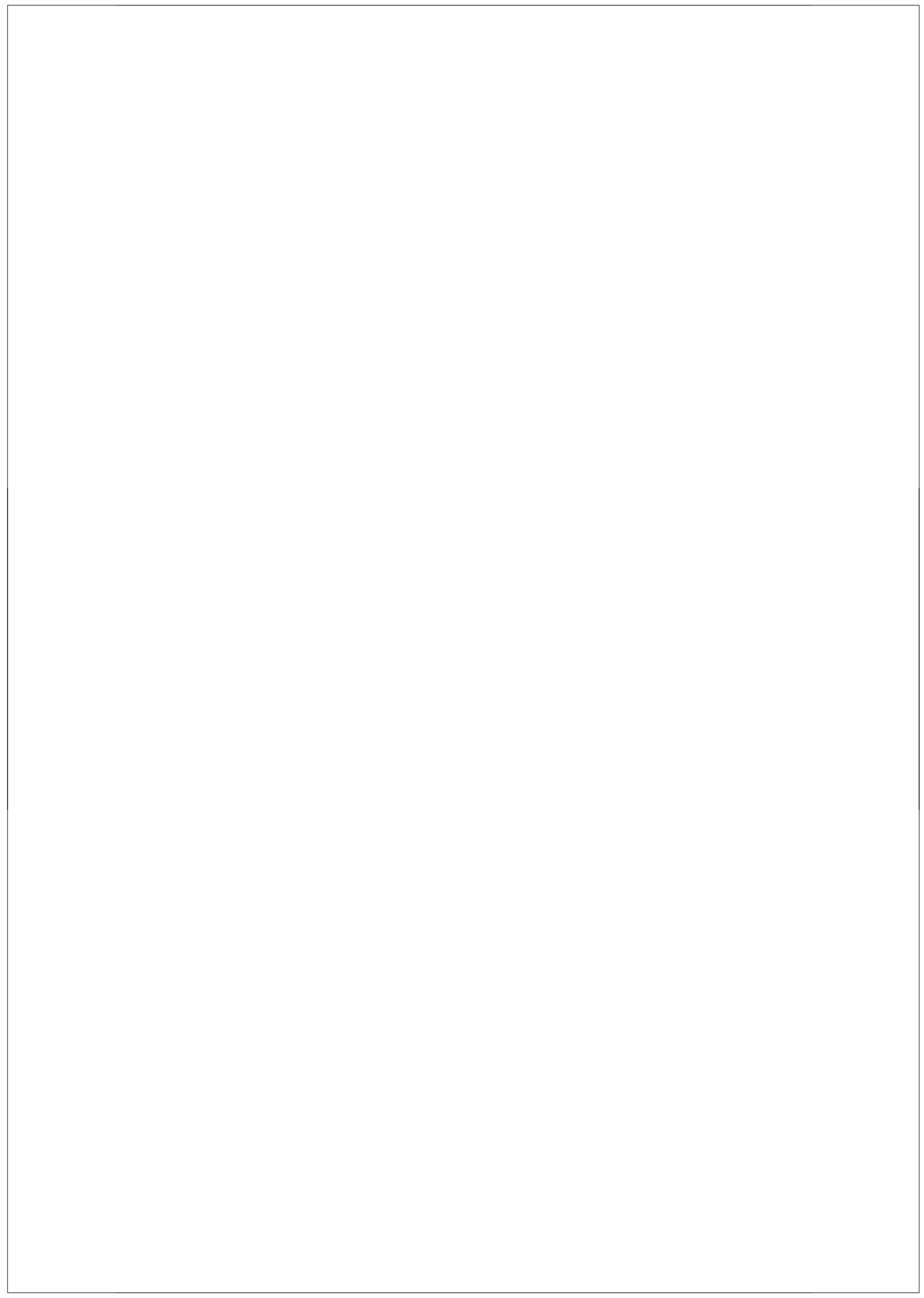




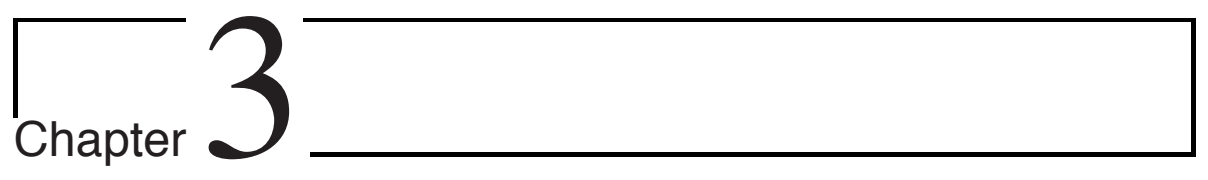

\section{Continuous-Time Delta-Sigma Modulators at High Sampling Rates}

This chapter describes the design of a continuous-time delta-sigma (CT $\Delta \Sigma)$ modulator that can achieve a $125 \mathrm{MHz}$ signal bandwidth $(\mathrm{BW})$ with a $70 \mathrm{~dB}$ dynamic range (DR) in $45 \mathrm{~nm}$ CMOS. As explained in the previous chapter, various system-level non-idealities (noise, non-linearity, metastability and excess loop delay (ELD)), will limit its performance. Especially for a modulator which targets a wide bandwidth, these limitations pose a major challenge.

In Section 3.1, we elaborate on the architectural design and system-level trade-offs of a $\mathrm{CT} \Delta \Sigma$ modulator operating at high sampling rates, where minimization of ELD and metastability are crucial. Furthermore, this section presents a high-speed capacitive feedforward loop filter architecture which overcomes the gain-bandwidth product (GBW) limitation associated with the use of a summation amplifier. Section 3.2 describes the block-level design requirements of the proposed modulator which are based on the architecture study presented in Section 3.1. These requirements are verified by system-level simulations which show the sensitivity of the designed modulator to many types of non-idealities. 


\subsection{System-Level Design}

\subsubsection{CT $\Delta \Sigma$ Modulator Design at High Sampling Rates}

In this work, the main challenge is achieving both a high DR and a wide signal $\mathrm{BW}$ with a CT $\Delta \Sigma$ modulator. To achieve the target DR, three requirements must be satisfied. The first is related to thermal noise and total harmonic distortion (THD), which have to be better than $70 \mathrm{~dB}$ in a $125 \mathrm{MHz} \mathrm{BW}$ and $-70 \mathrm{dBFS}$, respectively. The second is clock jitter, which, based on systemlevel simulations, requires clock buffers with less than $250 \mathrm{fsec}$ of jitter (rootmean-square (RMS)). The third, and most difficult, requirement is the need to maintain modulator stability while operating at a sampling frequency of $4 \mathrm{GHz}$. The first two requirements can be met by dissipating more power in the associated circuitry. However, the relationship between modulator stability and power consumption is more complex. For instance, a quantizer must generate a valid digital output within a fraction of a sampling-clock cycle to maintain modulator stability, which implies that more power must be dissipated at higher sampling frequencies. Similar requirements exist for the loop filter and the $\mathrm{DAC}$, since at $\mathrm{GHz}$ sampling rates, the delay associated with parasitic poles must be overcome by dissipating more power.

In an ideal delta-sigma modulator $(\Delta \Sigma \mathrm{M})$, the quantization error fundamentally defines the maximum achievable signal-to-noise ratio (SNR) in a given $\mathrm{BW}$. The signal-to-quantization noise ratio (SQNR) and BW of a single-loop $\Delta \Sigma \mathrm{M}$ depend on three main parameters: loop filter order, quantizer resolution, and sampling frequency $\left(f_{s}\right)$. Signal BW and $f_{s}$ are linked via the $O S R=f_{s} /(2 \times \mathrm{BW})$.

Figure 3.1 illustrates the relation between these three design parameters in a single-loop CT $\Delta \Sigma$ modulator. Each point in Fig. 3.1 is taken from simulation results and corresponds to an $80 \mathrm{~dB}$ SQNR in a $125 \mathrm{MHz} \mathrm{BW}$. It can be seen that achieving bandwidths in excess of $100 \mathrm{MHz}$ requires $\mathrm{GHz}$ sampling frequencies. A one bit quantizer can be clocked at a very high sampling frequency since its relaxed offset requirements lead to low area and small parasitic capacitances. For example, a $35 \mathrm{GHz}$ one bit $2^{\text {nd }}$ order modulator has been demonstrated in SiGe BiCMOS with a $55 \mathrm{~dB}$ DR in a $100 \mathrm{MHz}$ BW [1]. However, in currently available CMOS processes, such sampling frequencies are impractical. Moreover, for sampling frequencies greater than $30-40 \mathrm{GHz}$, the DR of the modulator will be limited by non-idealities such as clock jitter and quantizer metastability, as explained in Chapter 2.2 [2].

For the same SQNR, the required sampling frequency of a CT $\Delta \Sigma$ modulator can be reduced by increasing the resolution of the quantizer. However, the 


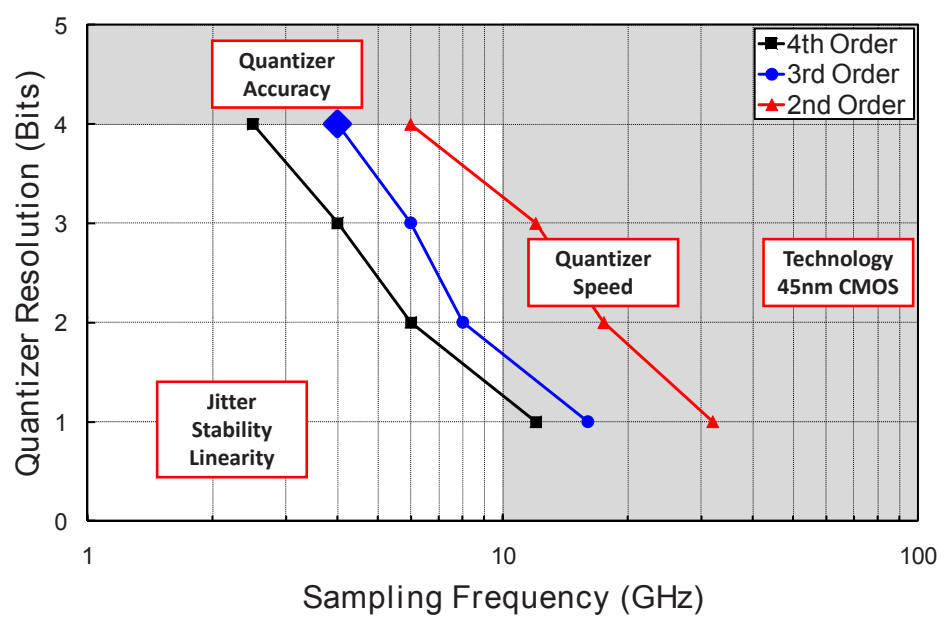

Figure 3.1 - System-level trade-off in a single-loop $\Delta \Sigma$ modulator for an $80 \mathrm{~dB}$ SQNR in a $125 \mathrm{MHz} \mathrm{BW}$.

maximum sampling frequency will then be limited by the quantizer's latency and the parasitic loop filter pole caused by its input capacitance. In practice, quantizers with resolution of up to 4-bits are used as a compromise between complexity, latency and the power dissipation in the clock distribution network [3-5]. For a given quantizer resolution, increasing the loop filter order also relaxes the sampling frequency. However, higher-order loop filters require more coefficients to stabilize the modulator. As the loop order increases, the oversampling ratio (OSR) decreases and the loop filter must deliver more gain to compensate for lower OSR. As a result, the coefficient scaling for modulators designed at low supply voltages can limit the possible architectural implementations. Moreover, the loop filter coefficients will drift due to process, voltage, and temperature (PVT) variations, and may cause SQNR degradation.

For a given sampling speed, decreasing the resolution of the quantizer while increasing the loop-filter order results in the same SQNR performance. However, as the resolution of the quantizer reduces, the LSB of DAC increases. As a result, the amount of clock jitter injected every time the DAC toggles increases, and the modulator becomes more sensitive to clock jitter, which is also verified by detailed system level simulations [6]. Since the error signal that the loop filter has to process then increases, the linearity requirement of the 


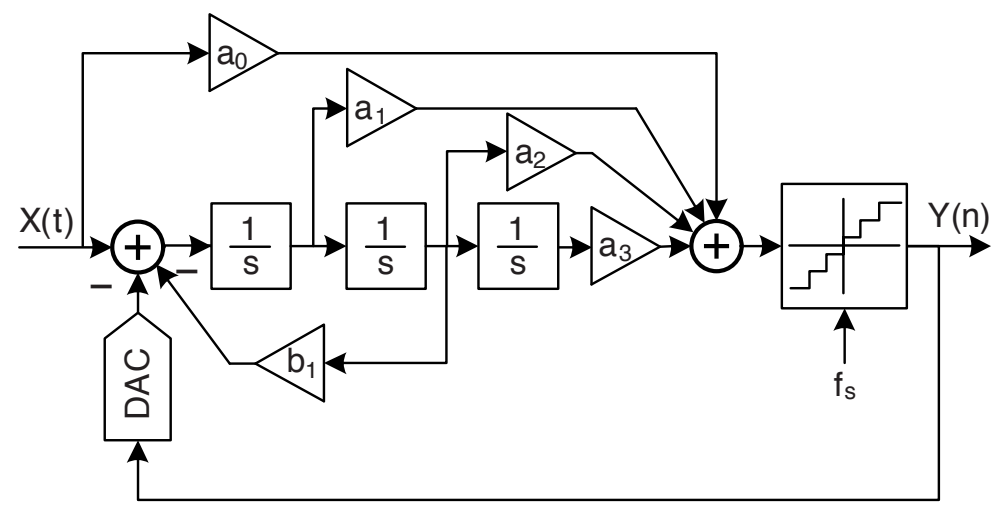

Figure 3.2 - A $3^{\text {rd }}$ order feedforward $\Delta \Sigma$ modulator.

integrator stages increases.

Despite the drawbacks of higher-order loop filters and multi-bit quantizers, they do facilitate a wide signal $\mathrm{BW}^{1}$. To meet the target specification of an $80 \mathrm{~dB}$ SQNR in a $125 \mathrm{MHz} \mathrm{BW}$, a $3^{\text {rd }}$ order single-loop modulator with a 4-bit quantizer sampled at $4 \mathrm{GHz}$ was chosen as shown in Fig. 3.2.

To minimize power consumption, the loop filter employs a feedforward loop filter instead of a feedback loop filter which requires more digital-to-analog converters (DACs) to implement feedback coefficients. At GHz sampling frequencies, these DACs significantly increase the power consumption and load the virtual ground of the amplifiers. To optimize the gain of the loop filter in the band of interest, a resonator is implemented around the first two integrators by using a local feedback coefficient $\left(b_{1}\right)$. As explained in Section 2.1.4, the signal transfer function (STF) of a modulator with a feedforward loop filter exhibits out-of-band peaking, which can be compensated by using a direct feedforward coefficient $\left(a_{0}\right)$ [8]. Furthermore, the direct feedforward coefficient relaxes the requirements on the loop filter's linearity. In the $45 \mathrm{~nm}-\mathrm{LP}$ process used, the choice of the modulator architecture was found to be a good trade-off between sampling frequency, clock jitter, linearity and circuit complexity. However, the use of a 4-bit quantizer is then the major limitation on the maximum achievable sampling rate, due to its delay and input capacitance.

\footnotetext{
${ }^{1} \mathrm{MASH} \Delta \Sigma$ modulators offer another route to increase signal BW [7]. However, the total output signal BW still depends on the signal BW of a single-loop modulator. Although this work focuses on extending the signal BW of a single-order modulator, the results can also be applied to increase the signal BW of MASH modulators.
} 


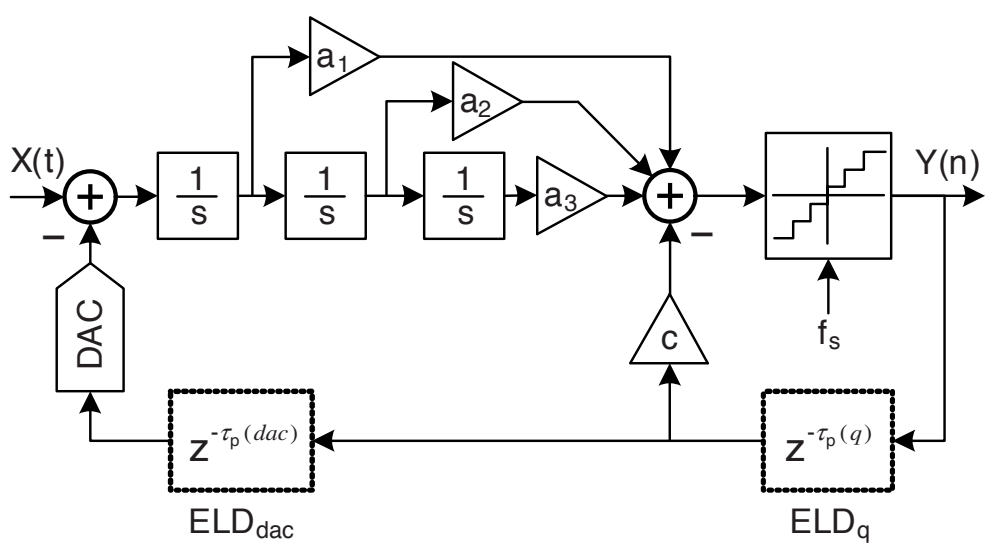

Figure 3.3 - A $3^{\text {rd }}$ order feedforward $\Delta \Sigma$ modulator with its ELD compensation.

\subsubsection{Excess Loop Delay Compensation with an Active Amplifier}

In order to design a stable modulator which is sampled at $4 \mathrm{GHz}$, the excess delay of the modulator must be compensated. Both the quantizer and DAC contribute to the ELD of the modulator, as illustrated in Fig. 3.3. The direct feedforward path $\left(a_{0}\right)$ and local feedback $\left(b_{1}\right)$ around the first two integrators are omitted throughout the section since they are mostly active in the signal bandwidth and do not affect the stability. As explained in Section 2.2.3, the ELD of the modulator is compensated for by an additional feedback path (c) and a summation node to preserve the noise transfer function (NTF) of the modulator. In this work, half a clock delay is allocated for the quantizer and DAC, which simplifies the clocking of the modulator.

To maintain the NTF and satisfy the stability requirements of the modulator, the summation node should not introduce additional ELD. A summation node can be implemented by the use of an active summing amplifier. The transfer function of an inverting summing amplifier can be expressed as:

$$
V_{\text {out }}(s) \cong-a_{i} V_{i}(s) \frac{1}{1+1 / A(s)}
$$

where $a_{i}$ is the scaling coefficient and $A(s)$ is the transfer function of the amplifier. The amplifier can be modeled as a single-pole system with a finite 
GBW:

$$
A(s)=\frac{A_{D C}}{1+s / \omega_{p}} .
$$

The limited gain of the summing amplifier acts as a fixed attenuation in the loop and reduces the effective gain of the loop filter. Therefore, the unity gain frequency of the loop filter shifts to a lower frequency, and the effective gain in the signal BW decreases. As a result of this, the SQNR of the modulator degrades.

To illustrate the effect that limited gain has on the summing amplifier, the behavior of a $3^{\text {rd }}$ order $\Delta \Sigma \mathrm{M}$ is simulated as shown in Fig. 3.3. The ELD of the quantizer $\left(\tau_{p}(q)\right)$ and the ELD of the DAC $\left(\tau_{p}(d a c)\right)$ are set to half a clock delay $\left(0.5 T_{s}\right) . \tau_{p}(q)$ and $\tau_{p}(d a c)$ are modeled with a discrete time delay, as shown in Fig. 3.3. As a first step to analyze the non-idealities of the summing amplifier, its gain is scaled down while $\omega_{p}$ is set to infinity. Figure 3.4a shows the NTF as a function of the $A_{D C}$ of the amplifier. The gain is varied from $40 \mathrm{~dB}$ down to $12 \mathrm{~dB}$. In this simulation, the OSR is 16 and the signal BW is shown as in Fig. 3.4a with a vertical dashed line. The effective loop-filter gain decreases as $A_{D C}$ decreases. As a results, the NTF starts peaking outside of the signal BW, which reduces the attenuation in the signal BW. Figure 3.4b shows the SQNR loss as a function of $A_{D C}$. As a consequence of a less effective NTF, the SQNR reduces with smaller values of $A_{D C}$. Furthermore, the peaking of the NTF close to the signal BW is not preferred since it increases the requirements of the decimation filter, which has to suppress the out-of-band quantization noise.

Figure 3.5 shows the maximum value of the integrators' output as a function of $A_{D C}$. In this simulation, we rely on the maximum value of the integrator' output instead of their RMS value because the $\Delta \Sigma \mathrm{M}$ is a non-linear system and any clipping can affect the stability. Therefore, to be on the safe side, this approach has been adopted.

In addition to SQNR loss, the integrators' output increases as $A_{D C}$ decreases. This can be solved by scaling down the feedforward coefficients $a_{1}, a_{2}$, and $a_{3}$. However, smaller feedforward coefficients increase the GBW requirement of the integrators. As a trade-off, an $A_{D C}$ of $20 \mathrm{~dB}$ can be chosen to limit the SQNR loss to $1-2 \mathrm{~dB}$ and to avoid a more than $10 \%$ increase in the output swings of the integrators normalized to the ideal output swings.

The limited BW of the summing amplifier acts as an additional pole in the loop and degrades the phase margin of the loop filter, which defines the stability of the modulator, as explained in Section 2.2.3. Figure 3.6a shows how a limited BW $\left(\omega_{p}\right)$ of the summing amplifier affects the NTF. The $A_{D C}$ is set 


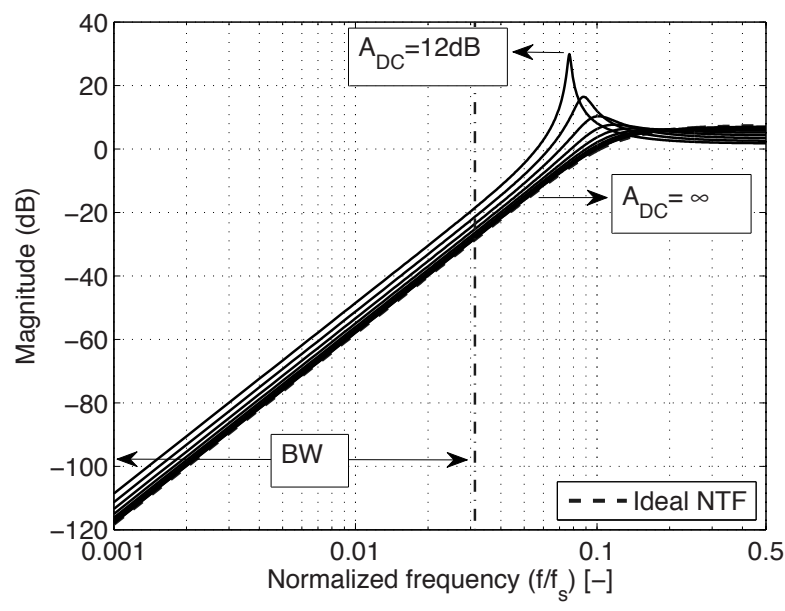

(a)

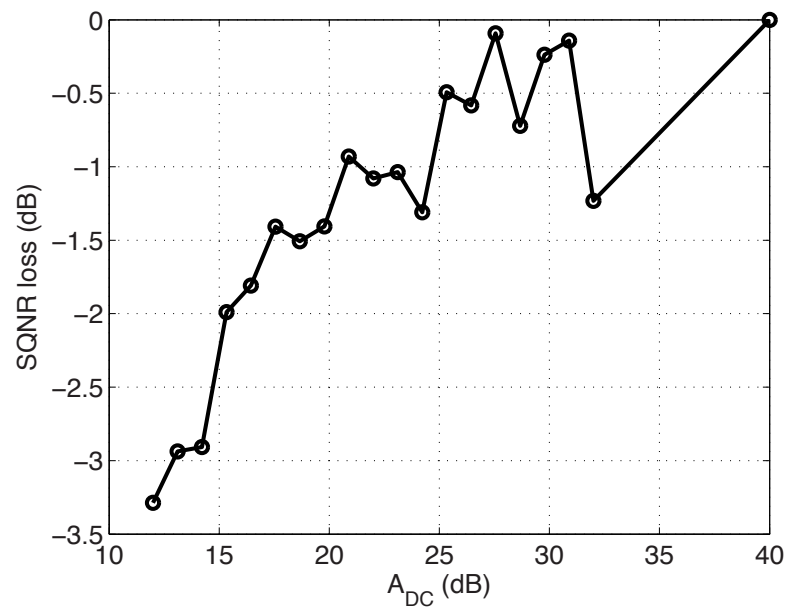

(b)

Figure 3.4 - NTF of the $3^{\text {rd }}$ order modulator with a non-ideal summing amplifier, where the DC gain of the amplifier is modeled as $A_{D C}$ (a). The SQNR loss of the modulator caused by the limited $A_{D C}(\mathrm{~b})$. 


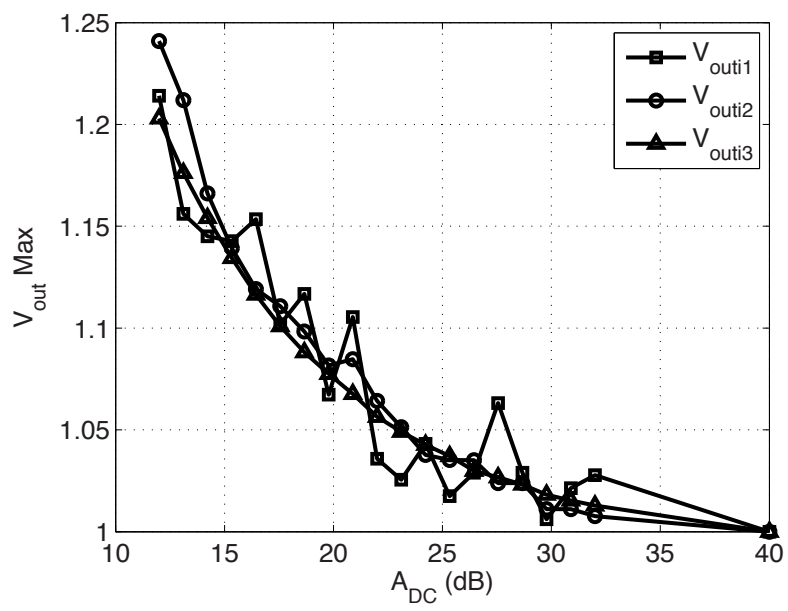

Figure 3.5 - The output of the loop-filter integrator stages, which is normalized to an ideal summation node, increases due to the limited $A_{D C}$.

to $20 \mathrm{~dB}$ and the frequency of the pole $f_{p}=\omega_{p} / 2 \pi$ is varied between $0.75 \mathrm{GHz}$ and $4 \mathrm{GHz}$. The ELD of the quantizer and DAC $\left(\tau_{p}(q)\right.$ and $\tau_{p}($ dac $\left.)\right)$ are set to half a clock delay $\left(0.5 T_{s}\right)$. Figure $3.6 \mathrm{~b}$ shows the zoomed-in version of the figure around the peaking of the NTF. The NTF (at $f_{p}=0.75 \mathrm{GHz}$ ) deviates from the NTF (at $f_{p}=4 \mathrm{GHz}$ ) by more than $2 \mathrm{~dB}$. As the pole frequency of the amplifier decreases, the maximum output state of the integrators increases as shown in Fig. 3.7, and for frequencies below $0.5 \mathrm{GHz}$, the modulator is unstable. Therefore, the $3^{\text {rd }}$ order modulator that is simulated in this design (Fig. 3.3) requires a summation amplifier with $A_{D C}>20 \mathrm{~dB}$ at $f_{p}>2 \mathrm{GHz}$ to minimize the effect of a finite GBW. This leads to a GBW product in excess of $20 \mathrm{GHz}$.

In addition to its limited GBW, the summation amplifier needs to drive a 4-bit quantizer, whose input capacitance introduces a secondary pole. Furthermore, the simulation results presented in Figs. 3.4 and 3.6 do not include the effect of other possible secondary poles which are present at the virtual ground node of the summing amplifier. These secondary poles can cause even more phase shift and can lead to instability. Therefore, the summation amplifier imposes very strict design requirements, which degrade the robustness of the modulator.

Figure 3.8 shows an alternative implementation in which the last integrator acts as a summation amplifier and adds the loop-filter coefficients and the feedback path around the quantizer [9]. The feedback path around the quantizer is 


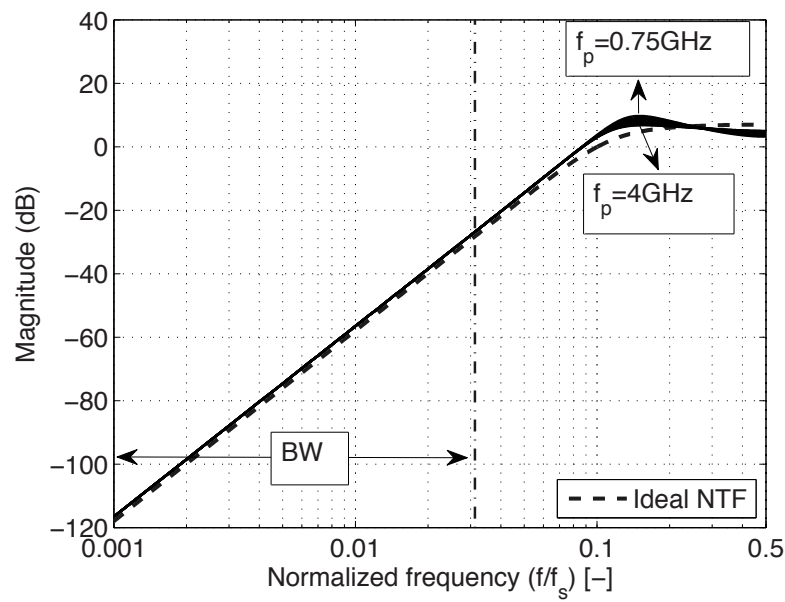

(a)

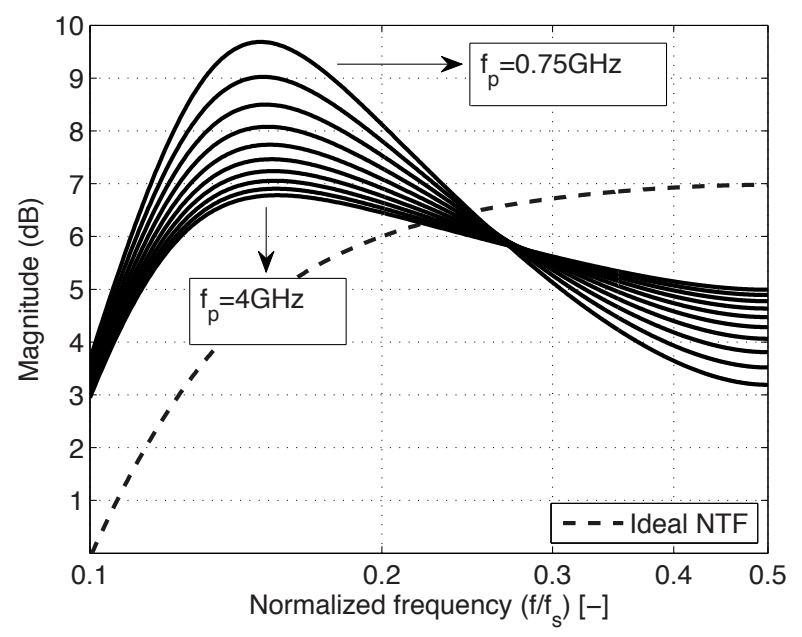

(b)

Figure 3.6 - NTF of the $3^{\text {rd }}$ order modulator with a non-ideal summing amplifier with $A_{D C}=20 \mathrm{~dB}$ (a); the zoomed-in version around the peaking of NTF (b). 


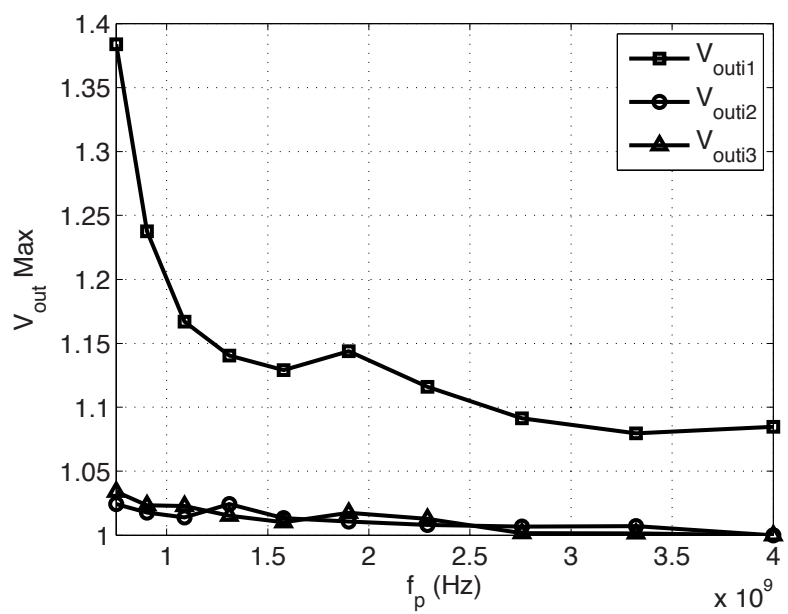

Figure 3.7 - The output states of the loop-filter stages normalized to a summation node for which $A_{D C}=20 \mathrm{~dB}$ and $\omega_{p}$ is infinite.

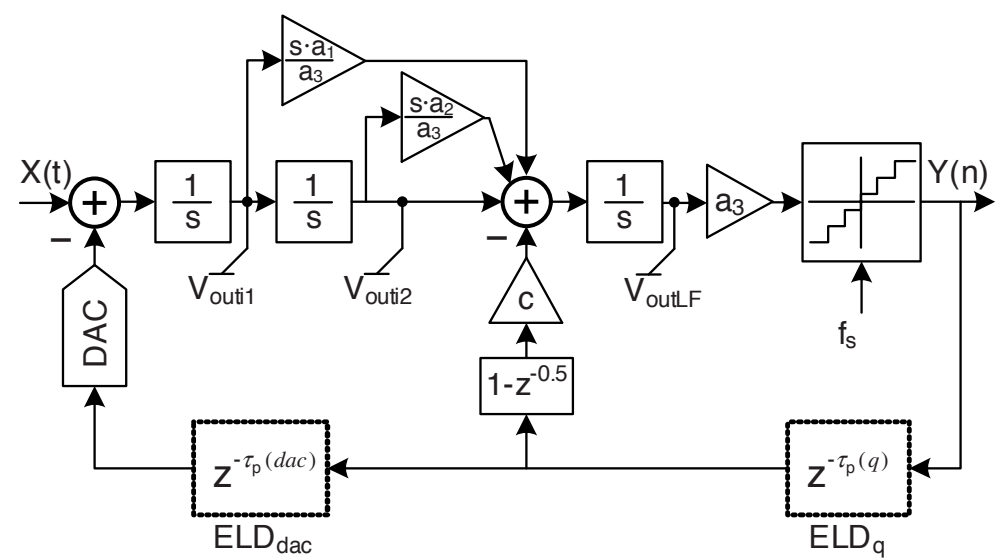

Figure 3.8 - A single-loop $\mathrm{CT} \Delta \Sigma$ modulator with last integrator acting as a summation node for differentiated signals 
differentiated in the digital domain, while the feedforward coefficients $a_{1}$ and $a_{2}$ are differentiated in the analog domain which allows the last integrator to act as a summation node. In this approach, an additional summation node is not necessary. The output of the last integrator acts as the output of the loop filter thus driving the quantizer.

Figure 3.9 shows the SQNR loss as a function of the $A_{D C}$ of the last integrator's amplifier shown in Fig. 3.8. Due to the limited DC gain of the amplifier, the SQNR reduces. Figure 3.10a shows the maximum output of the integrator stages. The output of the first and the second integrator increases while the feedback of the modulator keeps the output of the loop filter at the same level when compared to the ideal implementation. Figure 3.10b shows the output response of the integrator stages to the limited amplifier BW of the last integrator, where $A_{D C}$ is $20 \mathrm{~dB}$. The output of the first integrator increases directly as the $f_{p}$ decreases, since the path around the first integrator has a dominant role in the stability of the modulator. For pole frequencies $\left(f_{p}\right)$ smaller than $0.5 \mathrm{GHz}$ the modulator is unstable.

When compared to the previous case, the GBW requirement of the last integrator's amplifier is similar to that of the summing node amplifier even though the unity gain frequency of the last integrator is much less than the sampling frequency $\left(\omega_{3} \ll f_{s}\right)$. Therefore, from system-level simulations we have concluded that a $\Delta \Sigma \mathrm{M}$ with an active summation node requires amplifiers with $A_{D C}>20 \mathrm{~dB}$ at $f_{p}>1 \mathrm{GHz}$. This implies a unity-gain-bandwidth (UGBW) of greater than $10 \mathrm{GHz}$, which assumes that the amplifier is a simple one-pole system. Furthermore, the input capacitance of a 4-bit quantizer loads the output stage of the loop filter and increases the UGBW requirement of the amplifier. Therefore, the last amplifier which performs as a summation node is one of the major bottlenecks which limits the performance and maximum sampling speed of the modulator. The following section discusses a high-speed filter topology that overcomes these limitations and enables the use of $\mathrm{GHz}$ sampling frequencies.

\subsubsection{High-Speed Capacitive Feedforward CT $\Delta \Sigma$ Modu- lator}

Figure 3.11 shows the simplified architecture of the modulator that we will use as a first step to describe the proposed solution. The feedforward $\left(a_{0}, a_{1}, a_{2}\right.$, $\left.a_{3}\right)$ and local feedback $\left(b_{1}\right)$ coefficients are omitted for clarity. Since the active amplifiers create a bottleneck and limit the performance of the modulator, the proposed solution is to eliminate the active summation node and connect the loop filter directly to the quantizer. By implementing the last stage of the loop 


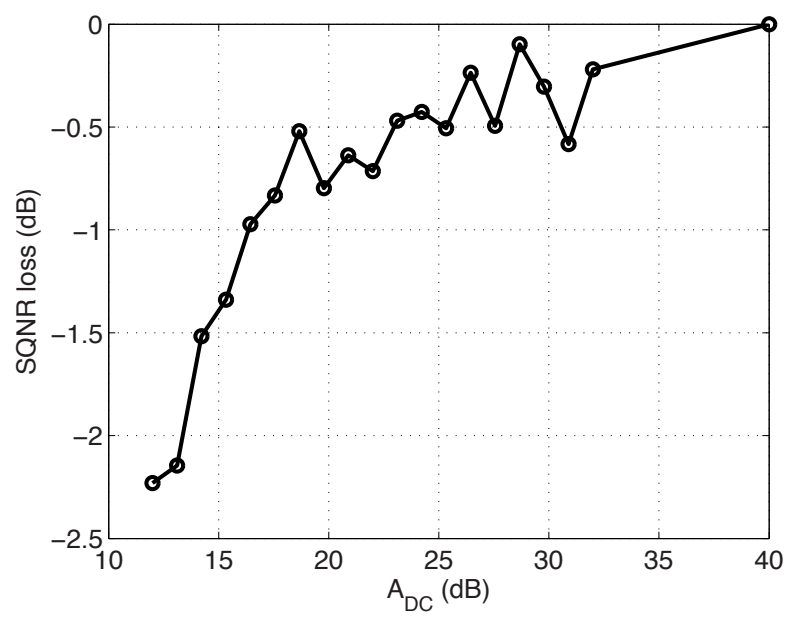

Figure 3.9 - The SQNR loss of the modulator with respect to $A_{D C}$.

filter as a transconductor, the quantizer's parasitic capacitance $C_{Q}$ can be used to realize one of the loop filter poles. The output current of the transconductor will then be directly integrated over $C_{Q}$ as shown in Fig. 3.11. To satisfy stability, however, there must still be a high-speed path around the quantizer, to compensate for its latency. As shown in Fig. 3.11, this can implemented with a current steering DAC (DAC2) that is driven by a digital differentiator $\left(1-z^{-\tau_{D A C 2}}\right)$, where $\tau_{D A C 2}=0.5 T_{s}[9]$. Due to the stability requirement of the modulator, the total delay around the quantizer and the main feedback DAC (DAC1) must not exceed one clock period as discussed in Section 2.2.3. Therefore, the following criteria must hold:

$$
\begin{aligned}
\tau_{D A C 2} & \leq T_{s}-\tau_{Q} \\
\tau_{D A C 1} & \leq T_{s}-\tau_{Q},
\end{aligned}
$$

where $\tau_{D A C 2}$ is the delay of DAC2, and $\tau_{D A C 1}$ is the delay of DAC1 (indicated as $T_{D}$ in the figure).

Figure 3.12 shows the block diagram of the proposed $3^{\text {rd }}$ order single-loop capacitive feedforward CT $\Delta \Sigma$ modulator where the implementation of the feedforward coefficients are shown. A feedforward topology requires a summation node for its feedforward coefficients. Since $C_{Q}$ can be used as a wideband passive summation node only for differentiated signals in the current domain, the feedforward voltages must be appropriately processed. This can be simply 


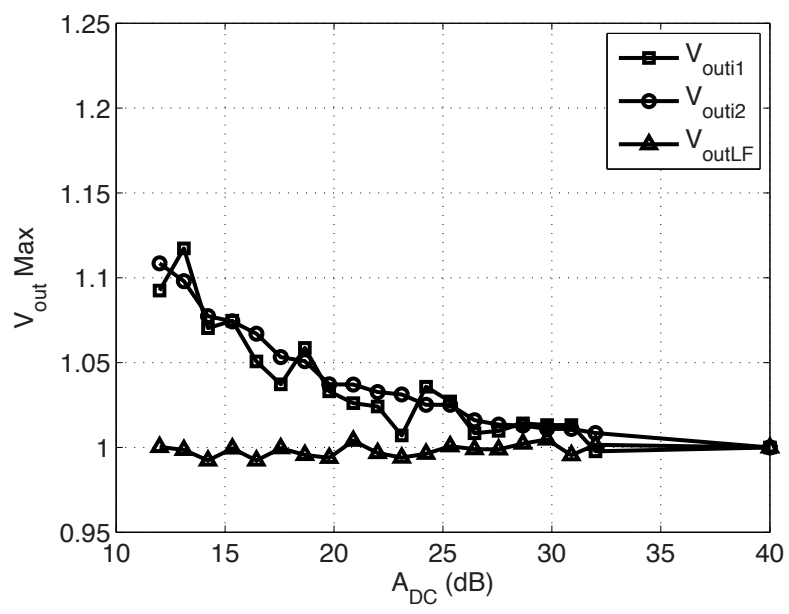

(a)

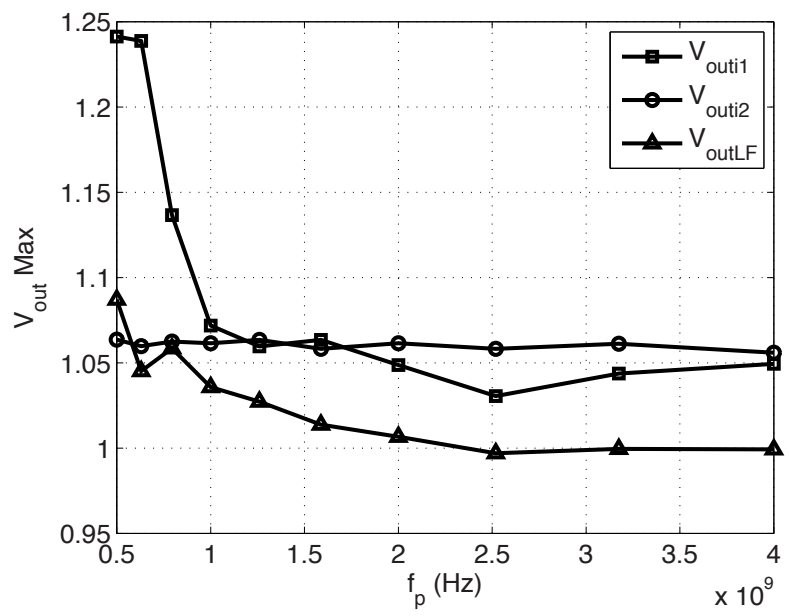

(b)

Figure 3.10 - The output states of the loop-filter stages normalized to an ideal summation node (a), and the output of the integrators normalized to a summation node for which $A_{D C}=20 \mathrm{~dB}$ and $\omega_{p}$ is infinite (b). 


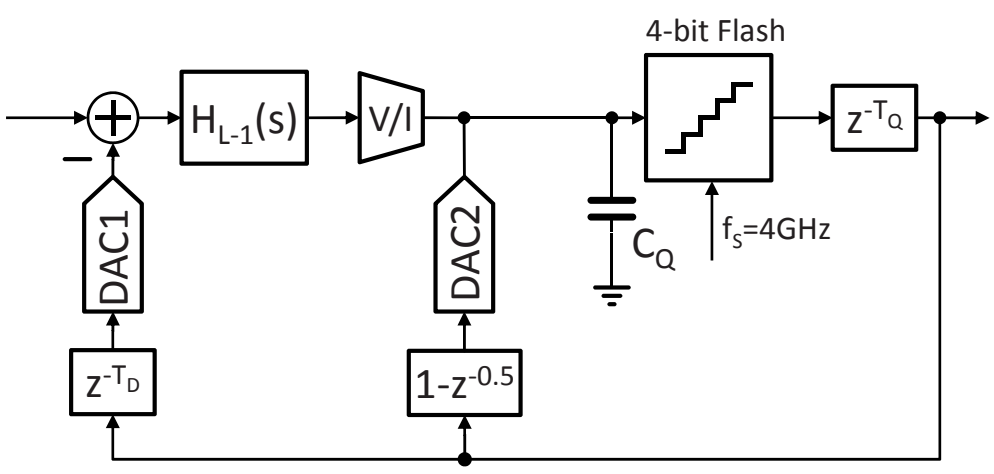

Figure 3.11 - A single loop $\Delta \Sigma$ modulator with a wideband summation node for differentiated signals in the current domain. The feedforward coefficients are omitted for clarity.

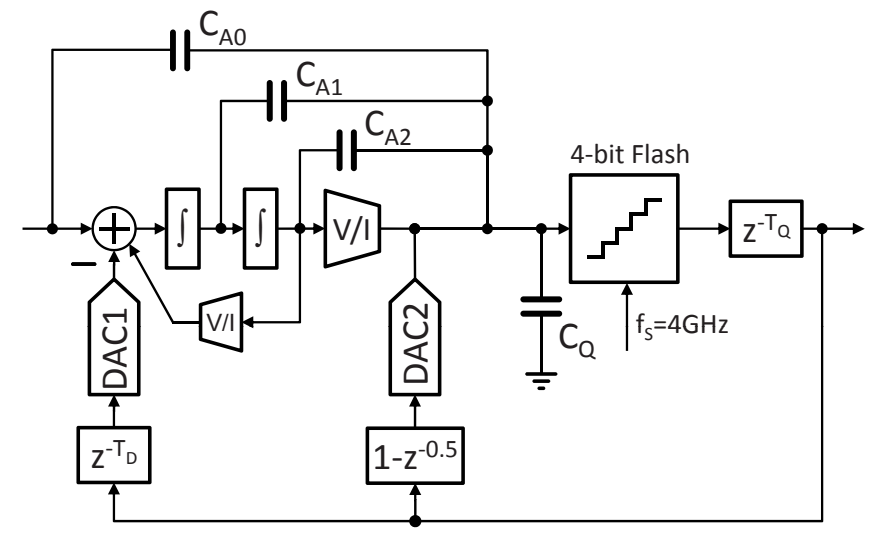

Figure 3.12 - The proposed high-speed capacitive feedforward CT $\Delta \Sigma$ modulator. 


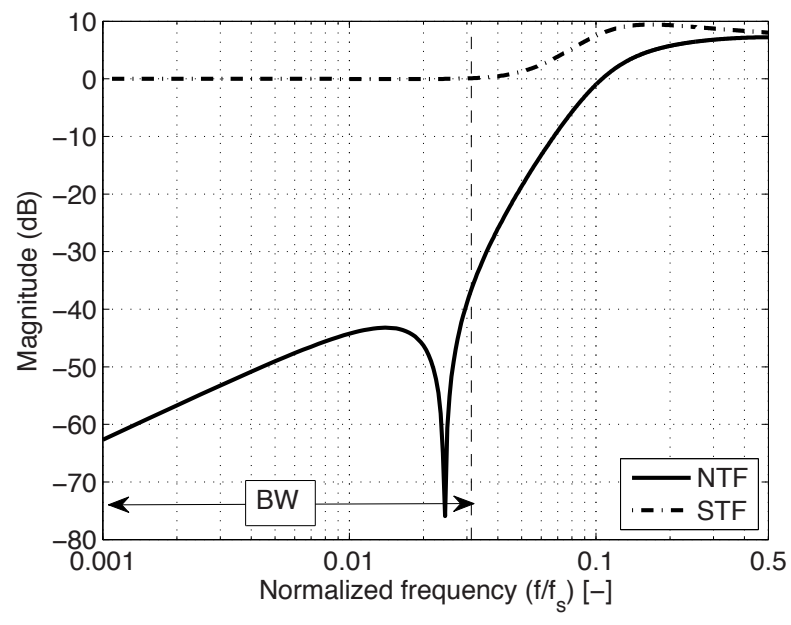

Figure 3.13 - The NTF and STF of the $3^{\text {rd }}$ order high-speed capacitive feedforward $\mathrm{CT} \Delta \Sigma$ modulator.

achieved by connecting capacitors $C_{A 1}$ and $C_{A 2}$ between the summing node and the outputs of the $1^{\text {st }}$ and $2^{\text {nd }}$ integrators. Furthermore, an overall feedforward path is implemented by $C_{A 0}$ to relax the requirements on the loop filter's linearity [8] and to reduce the peaking in the signal transfer function of the modulator at the cost of lower anti-alias filtering (Section 3.1).

Figure 3.13 shows the NTF and STF of the modulator. The NTF has a notch at DC and a complex notch located close to the edge of the signal BW which optimizes the in-band attenuation of the NTF. The out-of-band gain of the NTF is $7 \mathrm{~dB}$. The STF of a feedforward modulator often exhibits peaking which can be compensated by using a direct feedforward coefficient $(a 0)$. However, in the presence of one clock cycle $\left(T_{s}\right)$ ELD, the direct feedforward coefficient can no longer compensate for the out-of-band peaking at high frequency, and as a result, the STF exhibits approximately $8-9 \mathrm{~dB}$ of out-of-band peaking, as explained in Section 2.2.3. For cases in which the out-of-band peaking of the STF is important, the blocks which precede the modulator must have enough filtering to attenuate the unwanted signals sufficiently.

The feedforward coefficients can be expressed as:

$$
a_{n}=\frac{C_{A n}}{C_{T O T A L}},
$$

where $C_{T O T A L}\left(=C_{A 0}+C_{A 1}+C_{A 2}+C_{Q}+C_{D A C 2}\right)$ is the total capacitance 
Table 3.1 - The system-level noise budget of the $\Delta \Sigma \mathrm{M}$.

\begin{tabular}{|l|c|}
\hline SNR & Value $(\mathrm{dB})$ \\
\hline SNR $_{\text {Thermal }}$ & 72.0 \\
\hline SQNR & 80.0 \\
\hline SIGNAL-TO-JITTER-NOISE-RATIO (SJNR) $_{\text {S }}$ ( & 80.0 \\
\hline SNR $_{\text {Total }}$ & 70.8 \\
\hline
\end{tabular}

connected to the output of the loop filter. The feedforward capacitors $\left(C_{A 0}\right.$, $C_{A 1}, C_{A 2}$ ) are implemented by fringe capacitors. The total capacitance also includes the parasitic capacitances such as the input capacitance of the 4-bit quantizer $\left(C_{Q}\right)$ and the output capacitance of DAC2 $\left(C_{D A C 2}\right)$.The parasitic capacitances vary with the voltage swing present at the summing node. When compared to $C_{\text {TOTAL }}$, however the nonlinear part is negligible. The passive summation requires that $\left(a_{0}+a_{1}+a_{2}\right)=1-\left(C_{Q}+C_{D A C 2}\right) / C_{T O T A L}$, which can be guaranteed by design. The capacitive feedforward summation technique does not need any summation amplifiers and the BW of the passive capacitive summation node is thus high enough to support a $4 \mathrm{GHz}$ sampling frequency.

\subsection{Block-Level Design Requirements}

This section discusses the block level design requirements of the modulator. Table 3.1 summarizes the noise-budget breakdown of the modulator. This table was first derived from system-level simulations and then adjusted based on the implementation details (described in Chapter 4). The thermal noise of the modulator $\left(S N R_{\text {thermal }}\right)$ is dominated by the loop filter and DAC1, which are connected to the input of the modulator. As explained in the previous section, the required SQNR defines the OSR, the order of the loop filter, and the resolution of the quantizer, in other words, the architecture of the modulator. In addition, the SJNR defines the noise of the clock buffers and the architecture of DAC1 (through which the clock jitter is injected at the input of the modulator).

Figure 3.14 shows the system-level model used to derive the block-level requirements. Both in the schematic and the system-level model, the feedforward coefficients differentiate the signal which are then integrated by the last integrator. Similarly, the input to DAC2 is differentiated in the digital domain and integrated in the analog domain. Therefore, the passive summation node implemented by the capacitors in Fig. 3.12 is modeled as a summation node 


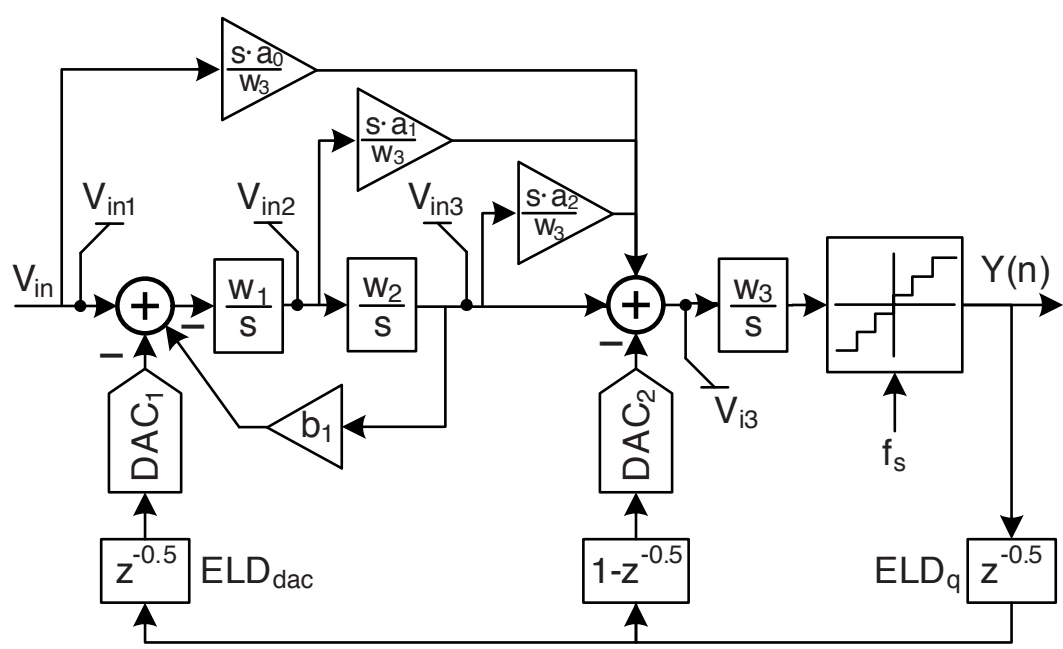

Figure 3.14 - Simplified block diagram of the feedforward CT $\Delta \Sigma$ modulator.

followed by the last integrator which adds the differentiated signals, as shown in Fig. 3.14.

\subsubsection{Loop Filter}

Figure 3.15 shows the input-referred transfer function of the integrators as illustrated in Fig. 3.14. The first integrator is directly connected to the input of the modulator and its non-idealities define the modulator's performance. On the other hand, non-idealities of the following stages are filtered by the preceding integrator stages. For example, the non-idealities of the second integrator are filtered by the first one, and at the edge of the signal BW, the non-idealities of the second integrator are attenuated at approximately $12 \mathrm{~dB}$. Moreover, the last integrator's non-idealities are filtered by the resonator. Therefore, the design requirements of the third integrator' are much more relaxed compared to those of the first and second integrator.

\section{Non-Linearity}

The linearity of the loop filter is defined by the first integrator. In order to meet the design linearity requirements, the first integrator can be implemented using an active-RC configuration, as shown in Fig. 3.16a. The unity gain frequency $\left(\omega_{1}\right)$ of the integrator is defined as $\omega_{1}=1 / R C$. The feedback around the 


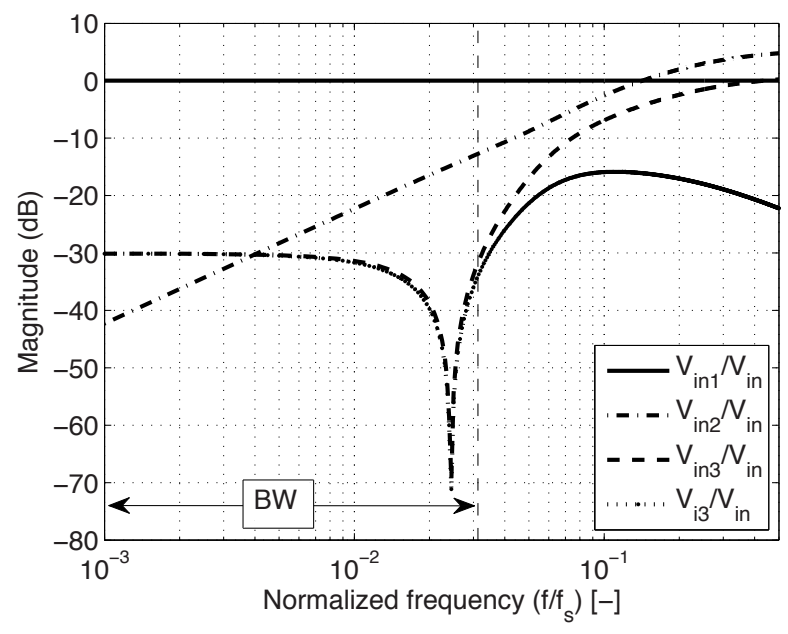

Figure 3.15 - The input-referred transfer functions of the $3^{\text {rd }}$ order high-speed capacitive feedforward CT $\Delta \Sigma$ modulator. Figure 3.14 shows the exact location of the probes.

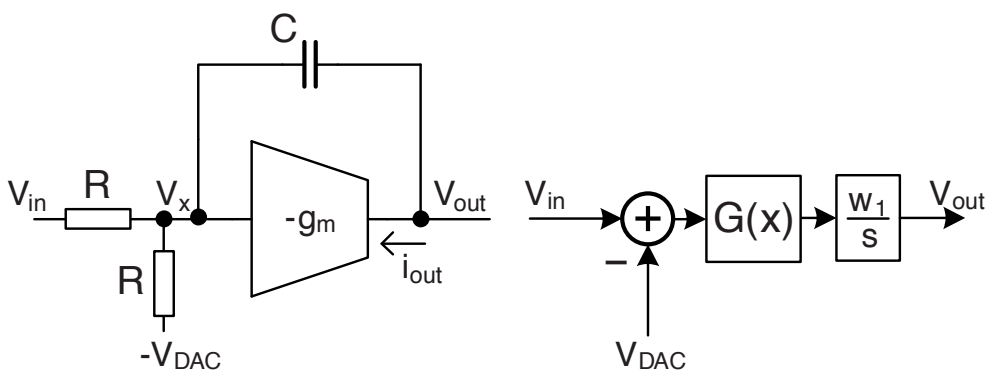

(a)

(b)

Figure 3.16 - The input stage of a $3^{r d} \mathrm{CT} \Delta \Sigma$ modulator based on active-RC implementation (a), and the system-level model with the non-linearity (b). 
amplifier improves its linearity, but, due to the limited transconductance $\left(g_{m}\right)$ of the amplifier, a signal swing exists at the virtual ground of the integrator, which is indicated as $V_{x}$ in Fig. 3.16a. As a result, the output current of the integrator can be expressed as $[10,11]$ :

$$
i_{\text {out }}=g_{m} V_{x}-g_{3} V_{x}^{3},
$$

where $g_{3}$ models the $3^{r d}$ order non-linearity of $g_{m}$. Throughout the following calculations, we assume that $g_{m} V_{x} \gg g_{3} V_{x}^{3}$. The signal and DAC input impedance are assumed to be equal to simplify the calculations, and applying the Kirchhoff's current law (KCL) at node $V_{x}$ results in:

$$
\frac{V_{i n}-V_{x}}{R}+\frac{-V_{D A C}-V_{x}}{R}=g_{m} V_{x}-g_{3} V_{x}^{3} .
$$

$V_{x}$ can be approximated as:

$$
V_{x} \approx \frac{V_{i n}-V_{D A C}}{2+g_{m} R}+\frac{g_{3} R\left(V_{i n}-V_{D A C}\right)}{\left(2+g_{m} R\right)^{4}}
$$

Assuming that the integrator is not loaded with another stage, its output is expressed as:

$$
V_{\text {out }}=-\int_{0}^{t} \frac{i_{\text {out }}}{C} d t .
$$

Substituting (3.7) in (3.5), the output of the integrator is expressed as:

$$
V_{\text {out }} \approx-\int_{0}^{t} \frac{g_{m}}{s C\left(2+g_{m} R\right)}\left(V_{i}-\frac{2 g_{3} V_{i}^{3}}{g_{m}\left(2+g_{m} R\right)^{3}}\right) d t .
$$

For $g_{m} R \gg 2, V_{\text {out }}$ then simplifies to:

$$
\begin{aligned}
V_{\text {out }} & =-\int_{0}^{t} G\left(V_{i}\right) \frac{1}{R C} d t, \\
G\left(V_{i}\right) & =\left(V_{i}-\frac{2 g_{3} V_{i}^{3}}{g_{m}\left(g_{m} R\right)^{3}}\right),
\end{aligned}
$$

where $V_{i}=\left(V_{i n}-V_{D A C}\right)$. By using Eq. 3.11, the non-linearity of the modulator can be modeled as shown in Fig. 3.16b [10], where the sign inversion of 
the integrator is omitted. The $3^{r d}$ order non-linearity of the integrator $(\beta)$ is expressed as:

$$
\beta=\frac{2 g_{3}}{g_{m}\left(g_{m} R\right)^{3}}
$$

The input to $G(x)$ consists of both the input signal and feedback signal from the DAC, which has a replica of the input signal and the shaped quantization noise. A part of the input signal is canceled by the feedback signal, yet a part of both the input signal and quantization noise is still present at the input to $G(x)$. Due to the non-linearity of the integrator, the loop filter not only introduces harmonic distortion but also mixes down the high-frequency quantization noise present at its input $[10,12]$. As a result, the non-linearity of the integrator increases the in-band noise of the modulator.

Figure 3.17 shows the signal-to-noise-and-distortion ratio (SNDR) and $3^{\text {rd }}$ order harmonic distortion $\left(\mathrm{HD}_{3}\right)$ of the $3^{\text {rd }}$ order modulator with a 4-bit quantizer as a function of the $3^{r d}$ order non-linearity $(\beta)$ of the first integrator. The analytical expression derived from (3.10) is also shown in the figure. A full-scale input signal at one third of the NTF notch frequency is applied to the modulator such that the $\mathrm{HD}_{3}$ tone due to the non-linearity is not masked by the quantization noise. As expected from (3.10), the $\mathrm{HD}_{3}$ tone is proportional to $\beta$ and for $\beta>0.1$ the SNDR also starts reducing, which implies that self mixing of the quantization noise due to the non-linear integrator increases the in-band noise of the modulator.

\section{Thermal Noise}

The $\mathrm{SNR}_{\text {Thermal }}$ of the modulator is $72 \mathrm{~dB}$, as listed in Table 3.1. Based on the system-level simulations, the SNR of the loop filter $\left(\mathrm{SNR}_{\mathrm{LF}}\right)$ is set to $80 \mathrm{~dB}$. Both the input impedance of the first integrator and its amplifier contribute to the noise. For a differential implementation, the $\mathrm{SNR}_{\mathrm{LF}}$ is expressed as:

$$
S N R_{L F}=10 \cdot \log _{10}\left(\frac{0.5 V_{i n}{ }^{2}}{2 v_{n, \text { Rin }}^{2}+v_{n, a m p}^{2}\left(1+\left(\omega / \omega_{1}\right)^{2}\right)}\right),
$$

where $v_{n, \text { Rin }}^{2}=4 k T R_{i n} B W, v_{n, a m p}^{2}=4 k T R_{e q} B W$, and $R_{e q}=2 / 3 g_{m}$ [13]. In most cases, $v_{n, \text { Rin }}^{2}$ is much greater than $v_{n, a m p}^{2}$ since the input transconductance of the amplifier is designed to meet the linearity or BW requirements of the modulator. 


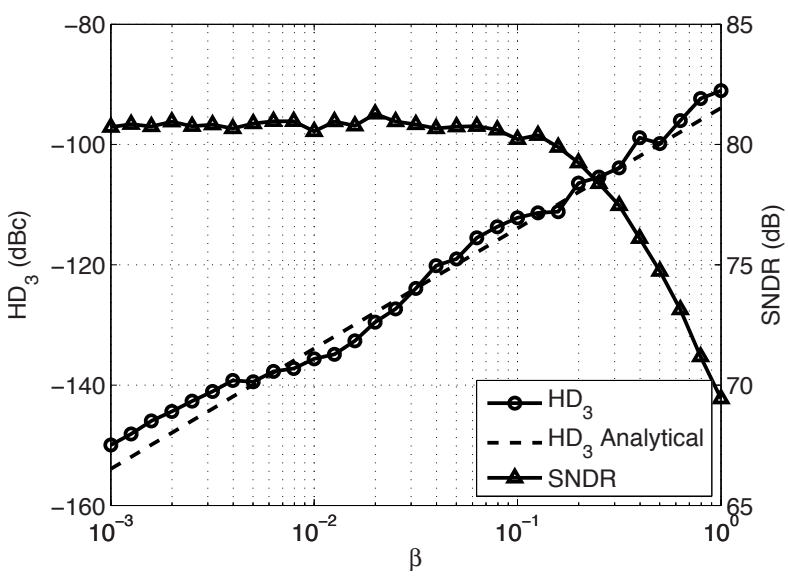

Figure 3.17 - SNDR and $\mathrm{HD}_{3}$ as a function of $3^{\text {rd }}$ order non-linearity $(\beta)$ of the first integrator; the FFT length is $2^{18}$ points and the input frequency is one third of NTF notch frequency.

\section{Finite GBW}

In addition to nonlinearity, the limited GBW of amplifiers reduces the effective gain of the loop filter and degrades the phase margin of the loop filter, which decreases the SQNR of the modulator. Furthermore, the effect of the limited GBW can be considered an ELD [14], which causes instability unless it is compensated for, as explained in Section 2.2.3.

Figure 3.16a shows an active-RC integrator, whose transfer function can be expressed as:

$$
I_{i}(s)=\frac{\omega_{i}}{s} \frac{1}{1+1 / A(s)+\omega_{i} /(s A(s))},
$$

where $\omega_{i}$ is the unity gain frequency of the integrator, and $A(s)$ is the transfer function of the amplifier. If the amplifier is a single-pole system, its transfer function is expressed as:

$$
\begin{aligned}
A(s) & =\frac{G B W}{s+\omega_{p}} \\
G B W & =A_{D C} \cdot \omega_{p}[\mathrm{rad} / \mathrm{sec}] .
\end{aligned}
$$

Figure 3.18a shows the SQNR loss of the modulator as a function of the finite DC gain of the amplifier $\left(A_{D C}\right)$ where the pole frequency is set to infinity. 


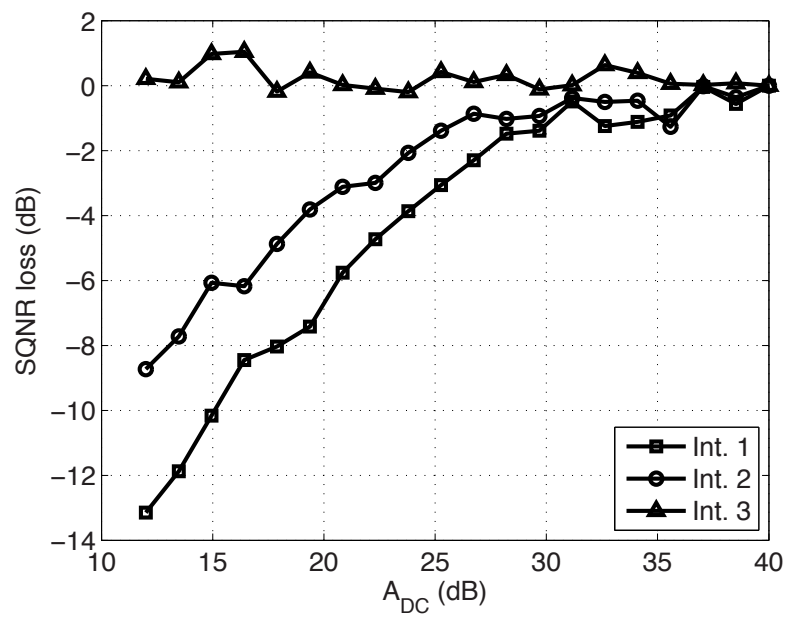

(a)

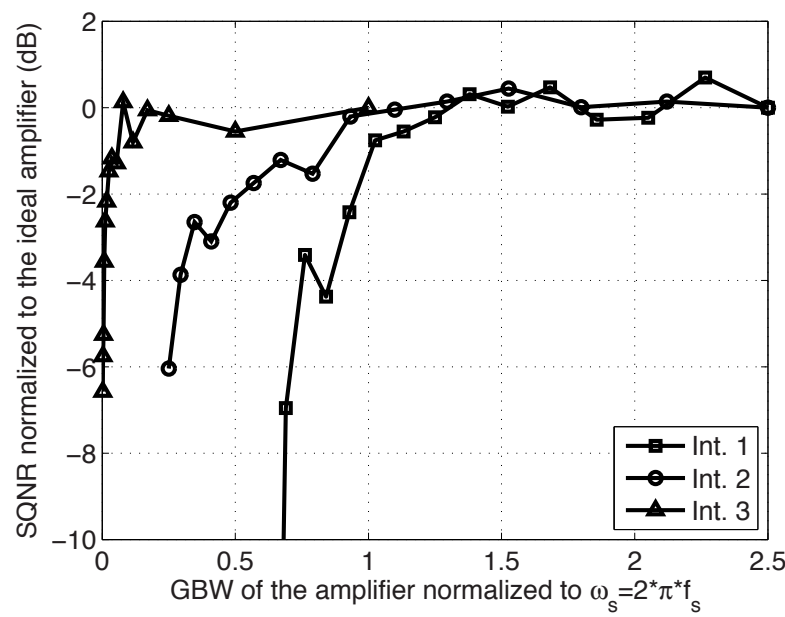

(b)

Figure 3.18 - The SQNR loss of the modulator due to the limited $A_{D C}$ of the amplifier (a), and the finite UGBW of the amplifier (b). 
In a feedforward $\Delta \Sigma \mathrm{M}$, the unity gain frequencies of the integrators often have a relation $\left(\omega_{1}>\omega_{2}>\ldots>\omega_{n}\right)$ which indicates that DC gain errors of the input stage have more impact on the SQNR. The first two integrators require $A_{D C}>35 \mathrm{~dB}$, and since the gain of the last integrator only defines the gain of the loop filter for very low frequencies, no considerable SQNR loss has been observed in the simulation results.

Figure $3.18 \mathrm{~b}$ shows the SQNR loss of the modulator as a function of the finite GBW normalized to the sampling frequency $\omega_{s}=2 \pi f_{s}$. The first integrator has the most stringent GBW requirement since any delay through the first integrator strongly effects the phase margin of the loop filter. This effect is less dominant in the second integrator. In architectures which implement a summation node at the virtual ground node of the last integrator, the last integrator has the highest GBW requirement [15]. However, in the proposed high-speed capacitive feedforward modulator architecture, this requirement is no longer necessary since the last integrator does not process the output signal of the ELD compensation DAC. Therefore, in this implementation the GBW of the last integrator is approximately $0.1 \omega_{s}$. This approach saves approximately one third of the power required for the loop filter. The designed GBW of the amplifiers is listed in Table 3.2.

Based on the analysis presented in this section, Table 3.2 summarizes the requirements of the blocks of the loop filter. The loop filter requires a phase margin of $80^{\circ}$ at $0.5 f_{s}$ due to the stability criteria explained in Section 2.1.4, and all the integrators require an approximate phase shift of $90^{\circ}$ at $0.5 f_{s}$. In order to reduce the SQNR loss to a minimum over PVT, the GBW of the integrators for a typical process corner is scaled $50 \%$ more when compared to the simulation results presented in Fig. 3.18b. The linearity of the first two integrators is set to $-80 \mathrm{dBc}$, which is well below the THD requirement of $-70 \mathrm{dBc}$. Since the last integrator's non-idealities are attenuated, only $\mathrm{HD}_{3}$ of $-30 \mathrm{dBc}$ is required. The next section explains the requirements of the quantizer, whose non-idealities are also attenuated by the NTF. However, the focus will be more on the sampling speed, which also defines the sampling rate of the modulator.

\subsubsection{Quantizer}

The speed of the quantizer is its most critical design requirement. Therefore, the preferred quantizer architecture is a flash ADC, as is also explained in Section 2.2.4. Figure 3.19a shows the architecture of the quantizer, which consists of 15 units. Each quantizer cell has a comparator and a DFF which holds the data for a one-clock period. Due to the strict latency requirements, 
Table 3.2 - Building-block specifications of the loop filter.

\begin{tabular}{|l|l|l|}
\hline Block & Specification & Value \\
\hline Sampling Speed & $f_{s}$ & $4 \mathrm{GHz}$ \\
\hline Input Signal & $V_{\text {in }}$ & $2 V_{p-p}$ \\
\hline \multirow{2}{*}{ Loop Filter } & Input Impedance & $1 \mathrm{k} \Omega$ \\
& Phase Margin & $80^{\circ} @ 0.5 \mathrm{f}_{\mathrm{s}}$ \\
& Total Harmonic Distortion $(\mathrm{THD})$ & $-80 \mathrm{dBc}$ \\
\hline Integrators & Phase Shift & $\approx 90^{\circ} @ 0.5 \mathrm{f}_{\mathrm{s}}$ \\
\hline \multirow{3}{*}{ OTA $_{1}$} & DC Gain $\left(A_{D C}\right)$ & $>35 \mathrm{~dB}$ \\
& GBW & $8 \mathrm{GHz}$ \\
& $\mathrm{HD}$ & $-80 \mathrm{dBc}$ \\
& $\mathrm{V}_{\text {noise }}$ & $-80 \mathrm{dBc}$ \\
\hline \multirow{3}{*}{ OTA $_{2}$} & DC Gain $\left(A_{D C}\right)$ & $>35 \mathrm{~dB}$ \\
& $\mathrm{GBW}$ & $6 \mathrm{GHz}$ \\
\hline \multirow{2}{*}{$\mathrm{OTA}_{3}$} & $\mathrm{~V}_{\text {noise }}$ & $-80 \mathrm{dBc}$ \\
\hline & $g_{m}$ & $0.5 \mathrm{x}-2 \mathrm{x}$ tunable \\
& $\mathrm{HD}$ & $-30 \mathrm{dBc}$ \\
\hline
\end{tabular}




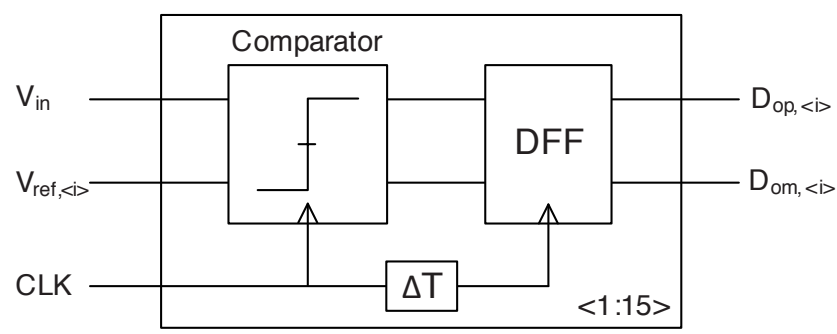

(a)

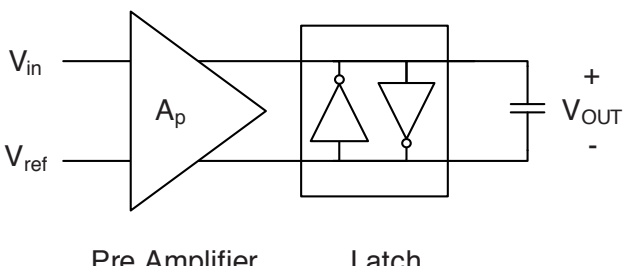

(b)

Figure 3.19 - Block diagram of the quantizer architecture (a), and the comparator (b).

the DFF is clocked with a delayed $(\Delta T)$ copy of the clock signal. Assuming that the DFF is faster than the comparator, clocking the DFF with a delayed copy of the clock signal reduces the metastability of the comparator as explained in the next sections.

\section{Metastability}

Figure $3.19 \mathrm{~b}$ shows the comparator, which consists of a preamplifier and a latch. At higher sampling speeds, the latch of the comparator suffers more from metastability. As a result, there is a finite chance that its output will not be defined. To meet the aimed DR, the errors introduced by metastability must be minimized. The metastability occurs when the latch is regenerating. During this phase, the latch can be modeled by two cross-coupled inverters. When the preamplifier of the comparator has an infinite BW and the latch starts its regeneration phase at $t=0$, the comparator's output can be expressed as $[16,17]$ :

$$
V_{\text {out }, i}(t)=V_{i} A_{\text {pre }} e^{t / \tau_{L}},
$$


where $V_{i}$ is the input voltage of the comparator $\left(V_{i}=V_{i n}-V_{\text {ref,i }}\right), A_{\text {pre }}$ is the gain of the preamplifier, and $\tau_{L}$ is the time constant of the latch:

$$
\begin{aligned}
& \tau_{L}=\left(\frac{1}{1-1 / A_{0}}\right) \frac{1}{2 \pi f_{u g b}} \\
& \tau_{L} \cong \frac{1}{2 \pi f_{u g b}}
\end{aligned}
$$

where $A_{0}$ is the gain of the latch, and $f_{u g b}$ is the UGBW of the latch. For this analysis, the gain of the latch is assumed to be $A_{0}>>1$.

To create a fully functional quantizer, each comparator is followed by a DFF such that the quantizer generates a valid digital output at $t=0.5 T_{s}$. If the DFF is edge-triggered, the output of the quantizer can be expressed as:

$$
V_{c o m p, i}(t)=\left(V_{i} A_{p r e} e^{\Delta T / \tau_{L}}\right) e^{(t-\Delta T) / \tau_{D F F}},
$$

where $\tau_{D F F}$ is the time constant of the DFF, which is expressed similar to (3.17), and $\Delta T$ is the delay between the between the comparator clock and the DFF clock as shown in Fig. 3.19a. If $\tau_{D F F}$ is equal to $\tau_{L},(3.18)$ simplifies to (3.16). However, for $\tau_{D F F}=\tau_{L} / \alpha$, where $\alpha$ is greater than 1 , the DFF acts as a gain stage and reduces the metastability of the quantizer.

As (3.18) indicates, if $V_{i n}$ is equal to one of the reference levels $\left(V_{\text {ref,i } i}\right)$, one of the comparators enters a metastability point, and its output is theoretically zero or undefined. On the other hand, we can derive the minimum input signal which generates a valid logic output $\left(V_{\text {logic }}\right)$ at $t=0.5 \cdot T_{s}$ as:

$$
V_{\text {min }}=\frac{V_{\text {logic }}}{A_{\text {pre }}} e^{-\frac{0.5 \alpha T_{s}-(\alpha-1) \Delta T}{\tau_{L}}}
$$

To simplify the calculation, we assume that noise can be neglected and the input of the comparator is uniformly distributed between $\pm V_{\min }$. Therefore, the probability of metastability of the quantizer slice can then be expressed as:

$$
P_{M, i}\left(t>0.5 T_{s}\right)=\frac{V_{\text {logic }}}{A_{\text {pre }}} e^{-\frac{0.5 \alpha T_{s}-(\alpha-1) \Delta T}{\tau_{L}}} .
$$

On the other hand, the quantizer has $2^{N}-1=15$ unit cells and the probability of the metastability of the quantizer is [17]:

$$
P_{M}\left(t>0.5 T_{s}\right)=\frac{P_{M, i}}{V_{L S B}}
$$


where $V_{L S B}$ is the quantization step size. The above analysis has been derived assuming that the metastability can only happen for a certain range of input signals. However, metastability cannot be restricted to input values smaller than a certain threshold voltage. In fact, there is always a small, but finite chance, that a meta-stable state occurs for any given input signal in the presence of noise [18]. Finally, the bit-error-ratio (BER) of the quantizer is expressed as $[16]$ :

$$
B E R_{Q}=\frac{P_{M, i}}{V_{L S B}}
$$

The expression in (3.22) indicates that the BER increases with the resolution of the quantizer. Furthermore, the criteria $\tau_{D F F}<\tau_{L}$ must be satisfied to minimize the BER of the quantizer. This is achieved since comparators in a multi-bit quantizer are often designed to meet the offset requirements, and the non-idealities of the DFF are attenuated by the gain of the pre-amplifier and latch. Therefore, small devices are used in the design of the DFF which can achieve a smaller time constant. As a result, it is logical to assume that the $\mathrm{DFF}$ is faster than the latch, which indicates that $\alpha>1$.

In addition, DAC1 is also clocked as shown in Fig. 3.12 and requires a DFF to retime the data of the quantizer. DAC1 clocked half a clock cycle after the quantizer generates its output signal. The output BER of the modulator is expressed as:

$$
B E R_{\Delta \Sigma M}=B E R_{Q} * e^{-0.5 T_{s} / \tau_{D A C 1}}
$$

The BER of the quantizer and the modulator are modeled in Fig. 2.26a and 2.26b, respectively. Section 2.2.4 explains the BER simulation in detail. Figure 3.20a shows the SQNR of the modulator as a function of the BER. The metastability errors introduced by the quantizer are shaped by the loop filter. The integrator outputs increase as the $B E R_{Q}$ increases, as shown in Fig. 3.20b. In this design, we aim to achieve a $B E R_{Q}$ of less than $10^{-5}$ to keep the increase in the integrators' maximum output voltage at less than $5 \%$ of its nominal value. On the other hand, as shown in Fig. 3.20a, the $B E R_{\Delta \Sigma M}$ degrades the SQNR dramatically. To achieve an SQNR higher than 80dB, in this design we aim for a $B E R_{\Delta \Sigma M}$ that is much smaller than $10^{-6}$.

Figure 3.21 shows the BER of the quantizer as a function of the $f_{u g b}$ of the latch based on (3.22). To achieve the aimed BER of $10^{-5}$, the $f_{u g b}$ of the quantizer must be greater than $6 f_{s}$. Reducing the trigger delay of the DFF $(\Delta T)$ improves the BER, but the transport delay of the comparator and kick back of the DFF must be simulated carefully, which will be discussed in the 


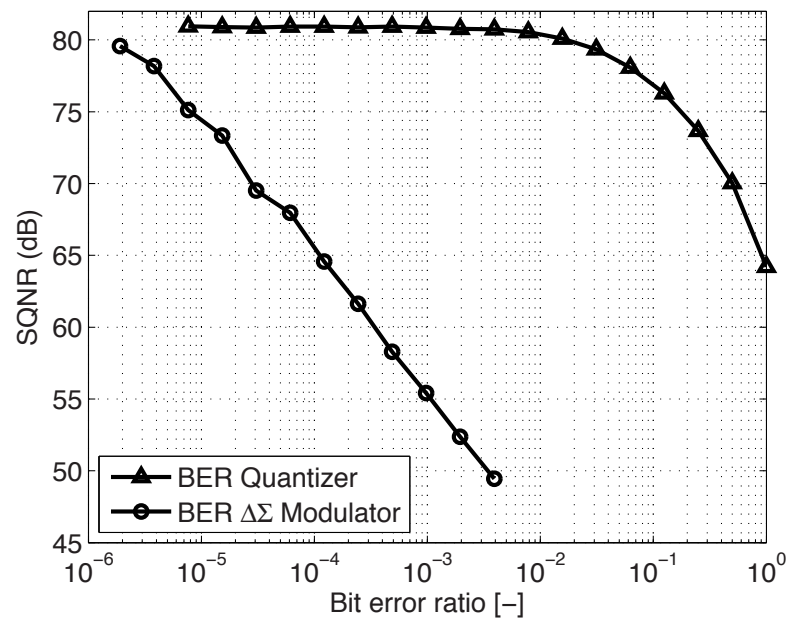

(a)

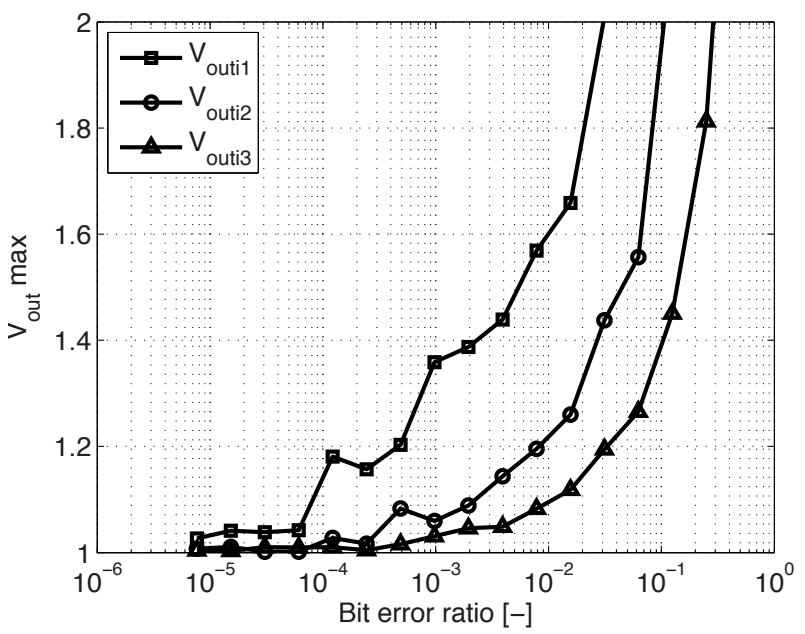

(b)

Figure 3.20 - The SQNR of the $3^{\text {rd }}$ order high-speed capacitive feedforward $\mathrm{CT} \Delta \Sigma$ modulator as a function of the bit error ratio (a), and the output of the integrator stages due to the BER of the quantizer (b). To estimate the effect of BER reliably, we ran ten simulations with random BER noise with a magnitude equal to the LSB. The minimum value of BER is limited by the simulation time. 


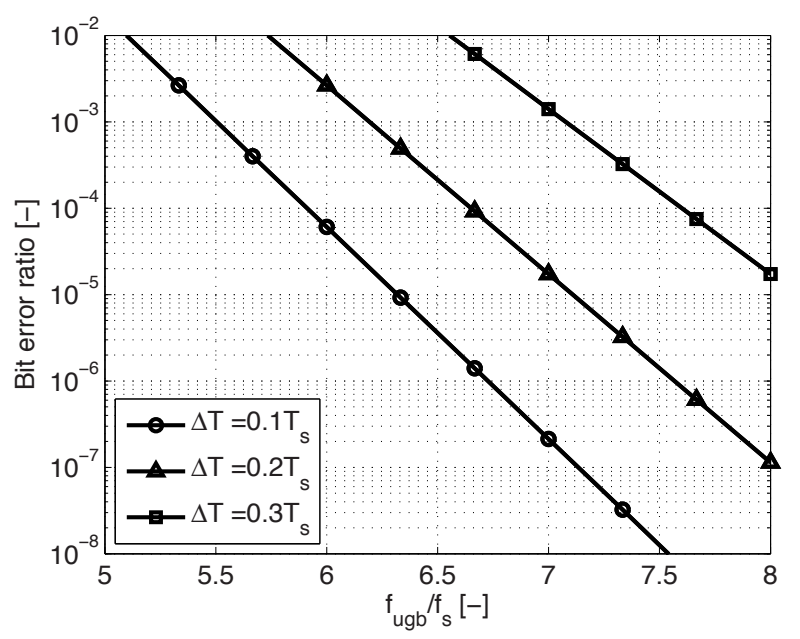

Figure 3.21 $-B E R_{Q}\left(t=0.5 T_{s}\right)$ as a function of $f_{u g b}$ of the latch normalized to the sampling frequency of the modulator. $\Delta T$ is the trigger instance of the DFF. The gain of the preamplifier $\left(A_{\text {pre }}\right)$ is $3[19]$, and $\alpha=\tau_{L} / \tau_{D F F}$ is set to 2 , assuming that the DFF is designed for speed rather than offset voltage.

next chapter. Since the DFF of the quantizer is used as the DFF of DAC1, the system-level simulations confirm that $B E R_{\Delta \Sigma M}$ is much lower than the $10^{-6}$ which is required to meet $70 \mathrm{~dB}$ of the DR.

\section{Non-Linearity}

Due to variation in the manufacturing process, each comparator has effectively a non-zero offset voltage $\left(V_{O S}\right)$. Figure 3.22 shows the SNDR of the modulator as a function of random $V_{O S}$. Even though the loop filter attenuates the nonlinearity caused by the $V_{O S}$ of the quantizer, the linearity of the modulator is still degraded as $\sigma_{V_{O S}}$ increases. In the simulations, the $\sigma_{V_{O S}}$ of the quantizer is set to $V_{L S B} / 8$, where $V_{L S B}$ is the quantization step size. Figure 3.23 shows the effect of quantizer offset on the SNDR and spurious-free dynamic range (SFDR) of the modulator, which relies on Monte Carlo simulations for the $\sigma_{V_{O S}}=V_{L S B} / 8$.

The noise introduced by the quantizer is shaped by the loop filter, therefore its noise contribution can be neglected. Table 3.3 summarizes the specifications of the quantizer. $0.5 T_{s}$ is allocated to the quantizer to keep the balance between the power dissipation of the ELD compensation and the quantizer. To meet 


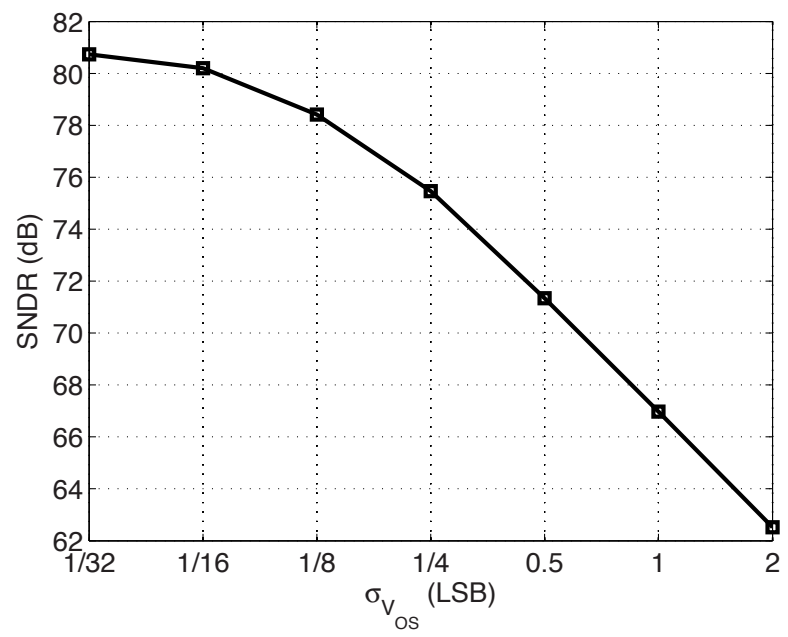

Figure 3.22 - SNDR as a function of $\sigma_{V_{O S}}$ of the quantizer normalized to its LSB. Each point on the figure is the average of 40 Monte Carlo simulations. The FFT size for each simulation is $2^{16}$ pts.

Table 3.3 - Specifications of the quantizer.

\begin{tabular}{|l|c|}
\hline Specification & Value \\
\hline Offset Voltage & $V_{L S B} / 8$ \\
\hline Latency & $0.5 T_{s}$ \\
\hline $\mathrm{A}_{\text {pre }}$ & 3 \\
\hline $\mathrm{f}_{\text {ugb }}$ & $6 f_{s}$ \\
\hline
\end{tabular}




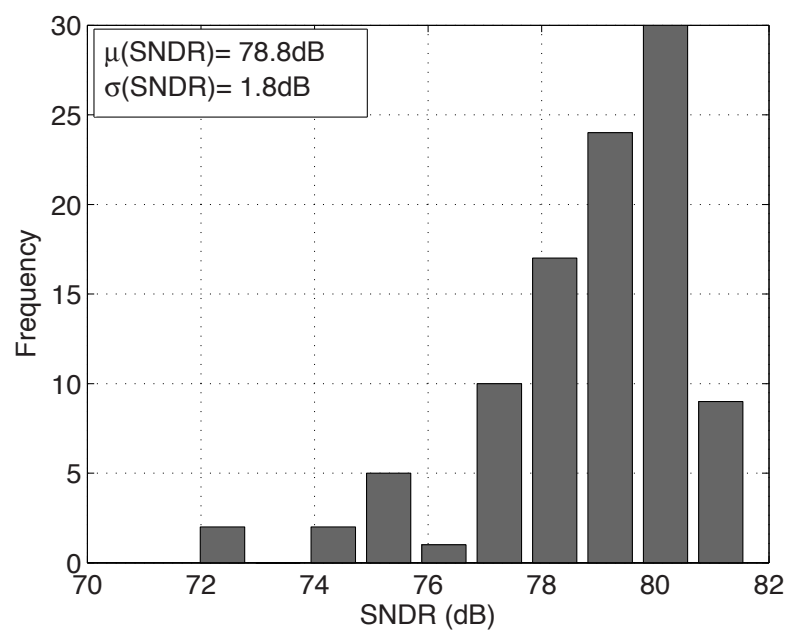

(a)

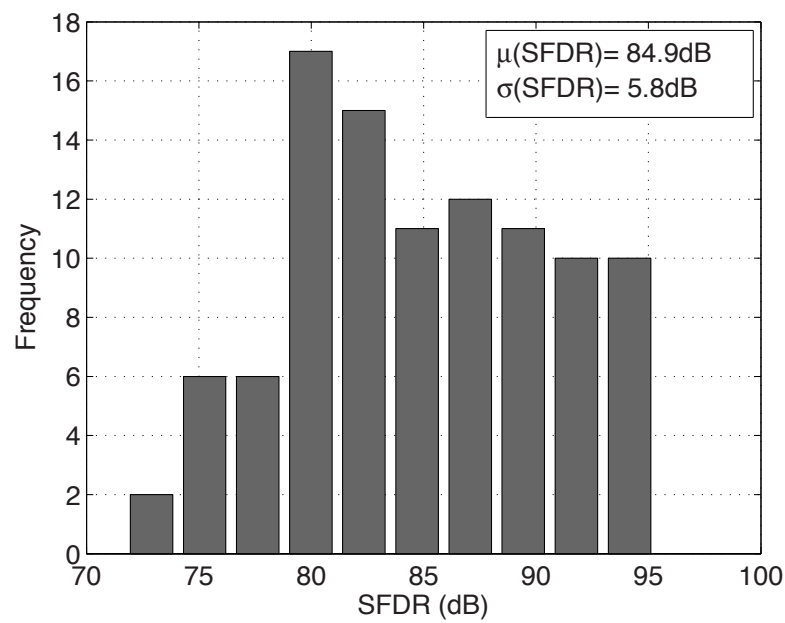

(b)

Figure 3.23 - The SNDR and SFDR of the modulator with offset voltage of the quantizer. 100 Monte Carlo runs are simulated. The comparator has a random offset voltage with $\sigma_{V_{O S}}=V_{L S B} / 8$. 


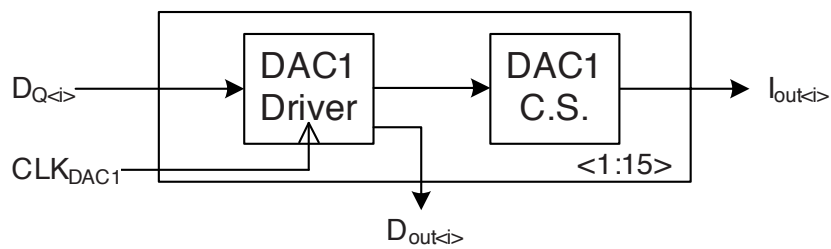

(a)

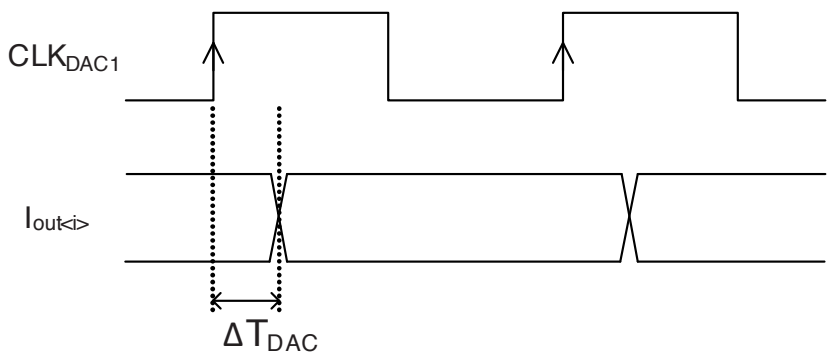

(b)

Figure 3.24 - The block diagram of DAC1 (a), the clocking of DAC1 and its output response

the latency requirement of the quantizer, the gain of the pre-amplifier must be $3 \mathrm{~V} / \mathrm{V}$ and the UGBW of the latch should be larger than $6 f_{s}$. All these speed requirements must be satisfied while meeting the offset requirement, which is set to $V_{L S B} / 8$.

\subsubsection{Feedback DAC (DAC1)}

Figure 3.24a shows the block diagram of DAC1, which is a multi-bit DAC with 15 unit sources. Each unit consists of driver circuitry and a current source. The main functions of the driver circuitry are to retime the data which drive the current sources and also to generate the output of the modulator, which is processed by the decimation filter. The additional latching functionality of the DAC driver reduces the metastability error to a sufficiently low enough level so that the modulator can achieve $70 \mathrm{~dB}$ of DR.

Figure 3.24b shows the clocking scheme of DAC1. At the rising edge of the clock, the data of DAC1 is captured, but the additional latching of the data

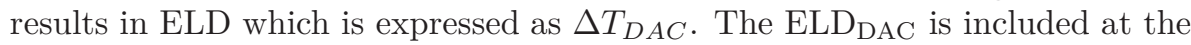
system level, as shown in Fig. 3.14, and can be compensated as explained in 


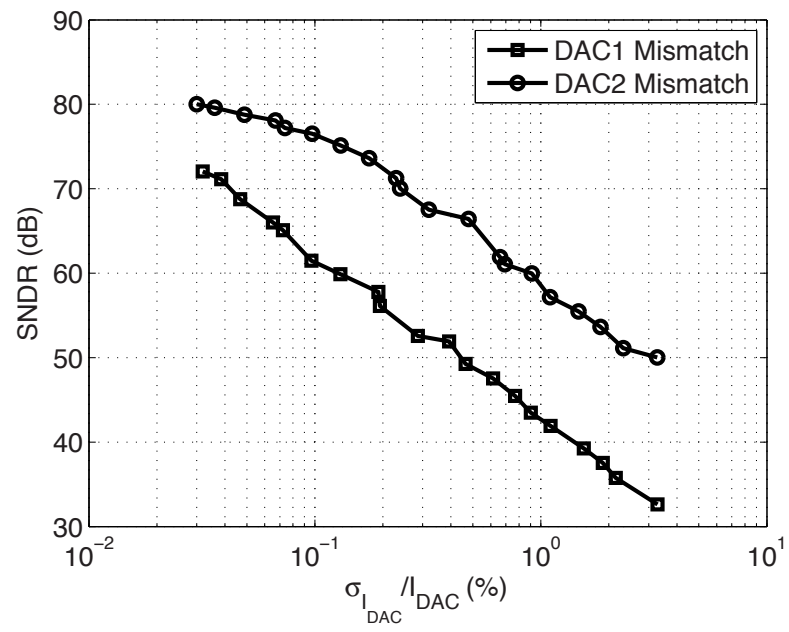

Figure 3.25 - SNDR as a function of DAC1 and DAC2 mismatch. Each point on the figure is the average of 40 simulations. The FFT size for each simulation is $2^{17}$ pts.

Section 2.2.3. The $\operatorname{ELD}_{\mathrm{DAC}}\left(\Delta T_{D A C}\right)$ must be smaller than $0.5 T_{s}$ to achieve a stable modulator.

\section{Non-Linearity}

DAC1 is the building block that defines the linearity, noise and jitter requirements of the modulator. Due to process variation and lithography errors, the output of each current source deviates from its originally designed value and creates static current mismatch which limits the low-frequency linearity. The DAC driver is implemented as a bank of DFFs. The device mismatch in the DFFs causes each DAC output pulse to have a different pulse width. As a result, the pulse width of the current output varies, leading to timing errors. For example, based on system-level simulations, a $0.1 \%$ random pulse width mismatch results in $-85 \mathrm{dBFS}$ of SFDR.

Figure 3.25 shows the mean value of the SNDR as a function of the static current mismatch of DAC1. As the mismatch increases, the SNDR of the modulator decreases. Various calibration techniques can be implemented to recover the SNDR. First of all, analog calibration can be used to tune the current sources of DAC1. The major advantage of this technique is that it does not introduce any ELD. If analog tuning of the current sources is not possible, 
digital correction techniques are preferred [20] that correct the errors introduced by DAC1 at the output of the modulator, as long as there is prior knowledge of the errors. Since the correction blocks are outside of the loop, no excess delay is introduced. However, processing the high speed digital data increases the power consumption of the modulator. Moreover, the power consumption of the digital correction strongly depends on the correction range. Yet, both the analog tuning and the digital correction require exact knowledge of the mismatch to improve the linearity. Therefore, on-chip measurement circuitry is required to extract the values of mismatch. On the other hand, data weighted averaging (DWA), which does not require exact knowledge of mismatch, can recover the SNDR, although is not very effective at a low OSR [21]. For an effective DWA, each DAC unit must be used equally and the frequency of use should not depend on the input signal. However, the amount of averaging is less at a low OSR. Furthermore, especially at high sampling frequencies, DWA introduces excess delay comparable to the clock period which degrades the stability. Therefore, using DWA is not favorable to implement it at $4 \mathrm{GHz}$. Additionally, DWA introduces in-band tones due to the data-dependent rotation of unit sources [22].

The implementation presented in Chapter 4 does not employ any DAC correction or calibration techniques since its main aim is to achieve a wide signal BW and high DR. Therefore, the mismatch errors introduced by DAC1 can only be reduced by proper sizing of device dimensions. Increasing the device dimensions improves the matching, but results in higher capacitive loads which limit the maximum switching speed of the DAC and increase the power dissipation. In this design, the DAC current sources are designed to achieve 11 bits of matching, which is found to be a good compromise between matching and sampling speed. As shown in Fig. 3.25, 11 bits of matching $\left(\sigma_{I_{D A C}} / I_{D A C}=\right.$ $0.05 \%$ ) translate into approximately $68 \mathrm{~dB}$ of SNDR for a full-scale input signal.

\section{Thermal Noise}

The thermal noise of DAC1 is the most dominant noise source of the modulator. The input stage of the modulator is an active RC-integrator as shown in Fig. $3.16 \mathrm{a}$, and the SNR of the modulator due to the thermal noise of the DAC is expressed as:

$$
S N R_{D A C 1}=10 \cdot \log \left(\frac{0.5 V_{i n}^{2}}{2 i_{n, d a c 1}^{2} R_{i n}^{2} B W}\right),
$$

where $i_{n, D A C 1}(A / \sqrt{H z})$ is the RMS output current noise density of DAC1, $B W=\left(0.5 f_{s} / O S R\right)$ is the signal BW of the modulator, and $R_{\text {in }}$ is the input 


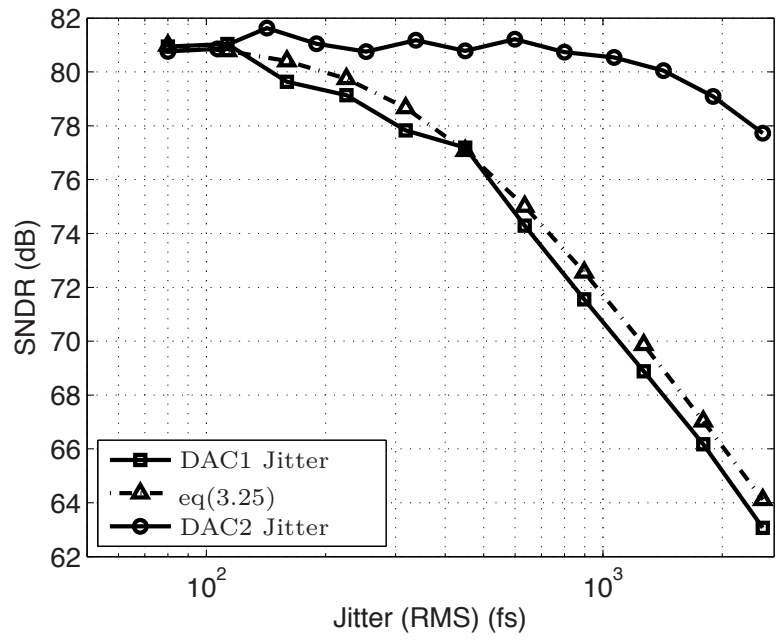

Figure 3.26 - SNDR of the $3^{\text {rd }}$ order high-speed capacitive feedforward CT $\Delta \Sigma$ modulator as a function of RMS clock jitter. For each simulation, the FFT size is $2^{17}$ pts.

impedance of the integrator. The meet $72 \mathrm{~dB}$ of $S N R_{\text {Thermal }}$ as listed in Table 3.1 , the $S N R_{D A C 1}$ is set to $73 \mathrm{~dB}$, which requires $0.01 n A / \sqrt{H} z$ of DAC1 output current noise density for $R_{\text {in }}$ equals to $1 \mathrm{k} \Omega$.

\section{Clock Jitter}

In addition to the thermal noise of the DAC units, $\mathrm{CT} \Delta \Sigma$ modulators especially with a current steering DAC are sensitive to clock jitter [23-25], which modifies the trigger moment of each DAC unit and effectively acts as an additional noise source at the input of the modulator. To minimize the performance loss due to the clock jitter, the clock distribution network and the digital circuits which drive the DAC must be designed to meet the SJNR.

Clock jitter can be modeled as an additional error which depends on the input signal amplitude and frequency. For a full-scale input signal, the SNDR of a lowpass $\Delta \Sigma$ modulator in the presence of clock jitter is expressed as [26]:

$$
\begin{aligned}
S J N R_{D A C 1} & =10 \log \left(\frac{O S R}{4 \sigma_{j}^{2} \gamma_{\text {dac }}^{2} f_{s}^{2}}\right) \\
S N D R & =10 \log \left(10^{-S J N R_{D A C 1} / 10}+10^{-S Q N R / 10}\right)
\end{aligned}
$$


Table 3.4 - Specifications of the DAC1.

\begin{tabular}{|l|l|}
\hline Specification & Value \\
\hline Matching & 11 bits \\
\hline Noise & $<0.01 n A / \sqrt{H z}$ \\
\hline Latency & $<0.5 T_{s}$ \\
\hline Jitter $(\mathrm{RMS})$ & $<250 \mathrm{fsec}$ \\
\hline
\end{tabular}

where $\sigma_{j}$ and $\gamma_{d a c}$ is the (RMS) value of the clock jitter, and the DAC output, respectively. In a single-bit modulator, the DAC output only toggles between " $\pm 1 "$ ", therefore, $\gamma_{d a c}$ is equal to 1 . However, the $\gamma_{d a c}$ of a multi-bit modulator depends on the aggressiveness of the NTF [27] and must be extracted empirically. As the resolution of the DAC increases, the $\gamma_{d a c}$ decreases, which improves the SJNR of the modulator. In other words, CT $\Delta \Sigma$ modulators with a multi-bit current steering DAC are less sensitive to clock jitter than their one-bit counterpart.

Figure 3.26 shows the SNDR as a function of the RMS value of the clock jitter where phase noise of the clock is modeled as a wideband white noise. Equation 3.25 and the simulation results are in good agreement, which relies on the empirically extracted $\gamma_{d a c}$. To achieve the aimed SNDR of $80 \mathrm{~dB}$, the RMS jitter of the driver and clocking circuitry of DAC1 must be smaller than 250 fsec.

Table 3.4 summarizes the specifications of DAC1. The linearity of DAC1 is set to 11 bits to achieve an SNDR of $68 \mathrm{~dB}$. The output current noise density of DAC1 is less than $0.01 n A / \sqrt{H z}$, which results in an SNR of $73 \mathrm{~dB}$. To satisfy the stability requirement, the latency of $\mathrm{DAC} 1$ is kept smaller than $0.5 T_{s}$, and to limit the noise due to clock jitter, the clock buffers and the DAC driver must have a RMS jitter of less than 250fsec. The clock source that drives DAC1 must also have a RMS jitter of less than 250fsec. Such a clock source can be implemented with a PLL which can generate $4 \mathrm{GHz}$ sampling clock. For example, such a PLL can be designed in $65 \mathrm{~nm}$ CMOS with less than $25 \mathrm{~mW}$ power dissipation [28].

\subsubsection{Quantizer DAC (DAC2)}

Figure 3.27a illustrates the block diagram of DAC2. Each DAC2 unit has two current sources and a DFF. The main function of DAC2 is to stabilize the modulator, therefore the latency of $\mathrm{DAC} 2$ is the most important design criterion. To reduce the latency, the differentiation $\left(1-z^{-0.5}\right)$ and DAC functionality 


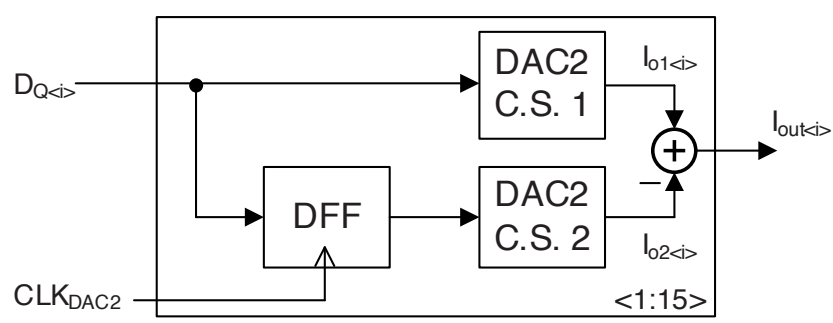

(a)

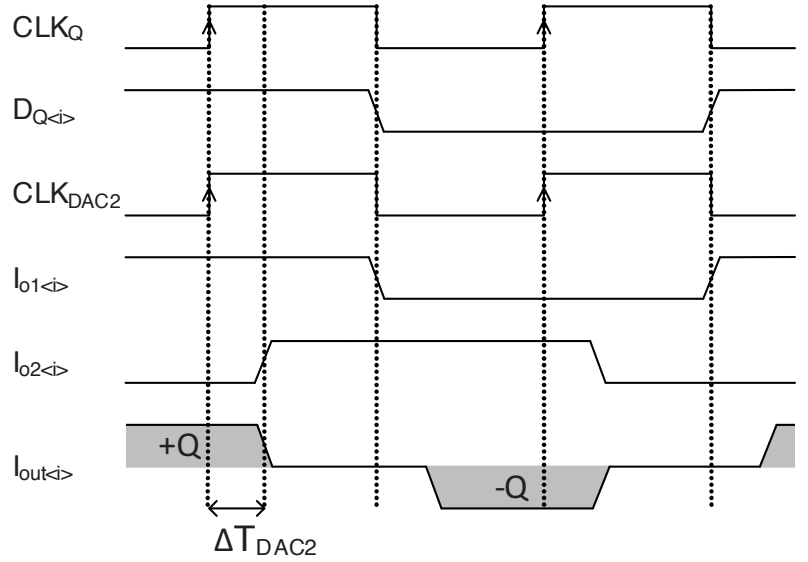

(b)

Figure 3.27 - Block diagram of DAC2 (a), and the timing diagram of DAC2 and its output response (b). 
are integrated into each DAC2 unit. Figure 3.27b shows the clocking scheme of DAC2. The output of each quantizer $\left(D_{Q<i>}\right)$ directly drives the first current source, which effectively reduces the ELD around the quantizer. At the rising edge of the clock $\left(C L K_{D A C 2}\right)$, the DFF resamples $D_{Q<i>}$, which is one clock cycle delayed compared to the sampling clock of the quantizer $\left(C L K_{Q}\right)^{2}$ However, the additional latching of the data results in ELD which is expressed as $\Delta T_{D A C 2}$. In other words, the output of DAC2 moves to the next sampling instance of the quantizer which adds an error proportional to $\left(Q \cdot \Delta T_{D A C 2} / T_{s}\right)$ to the input of quantizer. In order to overcome this limitation, the rising edge of $C L K_{D A C 2}$ is generated $\triangle T_{D A C 2}$ earlier compared to $\left(C L K_{Q}\right)$. As a result, the stability of the modulator is not degraded by the functional limitations of DAC2.

In an ideal differentiating DAC, the total number of current sources required depends on the maximum value of the derivative of the signal. The output of a multi-bit $\Delta \Sigma \mathrm{M}$ modulator only toggles a few LSBs. Since the main function of $\mathrm{DAC} 2$ is to stabilize the modulator, DAC2 is designed for minimum the latency. Therefore, DAC2 has two banks of current sources with 15 unit elements, which are connected to the output of the loop filter with reverse polarity. This architecture minimizes the latency of DAC2, but the matching requirement between two banks of DAC2 increases since all DAC units are utilized by the data stream. Especially, since DAC2 output must be zero during half of the clock period, which is required by the differentiation function $\left(1-z^{-0.5}\right)$. Due to the mismatch between two banks of current sources, DAC2 output $\left(I_{\text {out }}\right)$ is not zero, which mixes the quantization noise into the signal $\mathrm{BW}$ and reduces the SNDR of the modulator. If the output of DAC2 can be disconnected from the loop filter to realize an ideal zero, the matching and noise specification of DAC2 can be relaxed by one bit. Figure 3.25 shows the mean value of the SNDR as a function of the static output current mismatch of DAC2. The current sources of DAC2 are designed for nine-bit matching $\left(\sigma_{I_{D A C}} / I_{D A C}=0.2 \%\right)$, which results in an SNDR better than $70 \mathrm{~dB}$.

$\mathrm{DAC} 2$ is connected to the output of the loop filter. However, its nonidealities are shaped by the resonator of the modulator since it is effectively connected to the input of the last integrator, as shown in Fig. 3.14. The inputreferred transfer function of DAC2 is shown in Fig. 3.15 by the trace $V_{i 3}$. The gain of the resonator relaxes its requirements compared to those of DAC1. The meet the $72 \mathrm{~dB}$ of $S N R_{\text {Thermal }}$ noise budget listed in Table 3.1, the $S N R_{D A C 2}$ is set to $79 \mathrm{~dB}$, which requires $0.1 n A / \sqrt{H} z$ of DAC2 output current density.

Figure 3.26 shows the SNDR as a function of the RMS value of the clock jitter where phase noise of the clock is modeled as wideband white noise. DAC1

${ }^{2} C L K_{Q}$ signal in Fig. 3.27b and $R S T$ signal in Fig. 4.4 represent the same signal. 
Table 3.5 - Specifications of the DAC2.

\begin{tabular}{|l|l|}
\hline Specification & Value \\
\hline Matching & 9 bits \\
\hline Noise & $<0.1 n A / \sqrt{H z}$ \\
\hline Latency (DAC2 C.S. 2) & $<0.5 T_{s}$ \\
\hline Jitter (RMS) & $<1.5 \mathrm{psec}$ \\
\hline
\end{tabular}

has the most stringent jitter requirements, and the clock of DAC2 is a buffered version of DAC1 clock. To achieve the aimed SNDR of $80 \mathrm{~dB}$, the RMS jitter of the clocking circuitry of DAC2 must be smaller than 1.5psec. Table 3.5 summarizes the specifications of DAC2.

\subsection{Conclusions}

This chapter has presented the system level design of a $\Delta \Sigma \mathrm{M}$ with $125 \mathrm{MHz}$ signal BW and a $70 \mathrm{~dB}$ DR. In order to meet the design requirements, a $3^{\text {rd }}$ order single-loop modulator with a 4 -bit quantizer sampled at $4 \mathrm{GHz}$ has been chosen. This architecture is found to be a good trade-off between circuit complexity and sampling speed in the target fabrication process, which is 45-nm CMOS.

To achieve a stable modulator sampled at $4 \mathrm{GHz}$, the ELD of the building blocks is modeled at the system level. The drawbacks of the common ELD compensation techniques which rely on the virtual ground node of an active amplifier have been analyzed. The limited GBW of amplifiers degrades the SQNR, and the output of the integrator stages increases, thus reducing the available dynamic range for the signal processing. To overcome these limitations while maintaining power efficiency, a high-speed capacitive feedforward loop filter architecture has been proposed. This implements the summation node by employing a digital differentiated DAC whose output current is integrated on a capacitor, as explained in Section 3.1.3.

In addition, the system-level noise budget of the modulator has been presented, which defines the requirements of the building blocks of the modulator. The performance of the loop filter is limited by thermal noise, non-linearity of the first integrator, and the finite GBW of its amplifiers. The first integrator of the loop filter defines its thermal noise and non-linearity. A detailed analysis of the amplifier non-linearity has been presented which discusses harmonic tones generated by the non-linear $g_{m}$ of the amplifier. Moreover, the non-linear input stage of the loop filter self-mixes the quantization noise and reduces the SQNR. 
A multi-bit quantizer defines the maximum sampling frequency of the modulator. Therefore, a low latency flash comparator architecture has been designed. At high sampling speeds, the comparators suffer from metastability. A detailed analysis of metastability of quantizer in a $\Delta \Sigma M$ has been presented. Based on this analysis, the specifications of the quantizer have been derived. On the other hand, due to the non-zero offset voltage of each quantizer unit, the linearity of the modulator is limited even though the loop filter attenuates the non-idealities of the quantizer. The quantizer is dimensioned to meet a $\sigma_{V_{O S}}$, which is less than $V_{L S B} / 8$.

The feedback DAC (DAC1) has the most stringent design requirements since it is directly connected to the input of the modulator. The block-level design of DAC1 has been described and the effect of mismatch on the nonlinearity of the modulator has been simulated. DAC1 is designed for 11-bit matching, which is found to be a good compromise between sampling speed and matching since it also defines the area of DAC1. Moreover, DAC1 requires an output current noise density of less than $0.01 n A / \sqrt{(H z)}$ and clock jitter (RMS) smaller than 250fsec.

DAC2 of the modulator which is added to stabilize the modulator has much more relaxed design specifications when compared to DAC1. Its non-idealities are shaped by the resonator of the loop filter. DAC2 requires nine-bit matching to achieve a 70dB SNDR, and 1.5psec RMS clock jitter to achieve an $80 \mathrm{~dB}$ SNDR, which are derived from the system-level simulations.

The next chapter explains the circuit-level implementation details of the modulator, which are based on the results presented in this chapter. Furthermore, the measurement results of the implementation are presented in detail.

\section{References}

[1] A. Hart and S. Voinigescu, "A $1 \mathrm{GHz}$ bandwidth low-pass $\Delta \Sigma$ ADC with 20-50 GHz adjustable sampling rate," in Solid-State Circuits, IEEE Journal of, vol. 44, no. 5, pp. 1401-1414, May 2009.

[2] J. A. Cherry and W. M. Snelgrove, Continuous-Time Delta-Sigma Modulators for High-Speed A/D Conversion: Theory, Practice and Fundamental Performance Limits. Norwell, MA, USA: Kluwer Academic Publishers, 2000 .

[3] J. Arias et al., "A 32-mW 320-MHz continuous-time complex delta-sigma ADC for multi-mode wireless-LAN receivers," in Solid-State Circuits, IEEE Journal of, vol. 41, no. 2, pp. 339-351, Feb. 2006. 
[4] L. Breems, R. Rutten, R. van Veldhoven, and G. van der Weide, "A 56 $\mathrm{mW}$ continuous-time quadrature cascaded $\Sigma \Delta$ modulator with $77 \mathrm{~dB}$ DR in a near zero-IF $20 \mathrm{MHz}$ band," in Solid-State Circuits, IEEE Journal of, vol. 42, no. 12, pp. 2696-2705, Dec. 2007.

[5] S. Paton et al., "A 70-mW 300-MHz CMOS continuous-time $\Sigma \Delta$ ADC with 15-MHz bandwidth and 11 bits of resolution," in Solid-State Circuits, IEEE Journal of, vol. 39, no. 7, pp. 1056-1063, July 2004.

[6] M. Ortmanns and F. Gerfers, Continuous-Time Sigma-Delta A/D Conversion, Fundamentals, Error Correction and Robust Implementations. Springer, 2005.

[7] S. Norsworthy, R. Schreier, and G. Temes, Delta-Sigma Data Converters (Theory, Design, and Simulation). John Wiley and Sons, Inc, 1996.

[8] J. Silva, U. Moon, J. Steensgaard, and G. Temes, "Wideband lowdistortion delta-sigma ADC topology," in Electronics Letters, vol. 37, no. 12, pp. 737-738, June 2001.

[9] G. Mitteregger et al., "A 20-mW 640-MHz CMOS continuous-time ADC With 20-MHz signal bandwidth, 80-dB dynamic range and 12-bit ENOB," in Solid-State Circuits, IEEE Journal of, vol. 41, no. 12, pp. 2641-2649, Dec. 2006.

[10] P. Sankar and S. Pavan, "Analysis of integrator nonlinearity in a class of continuous-time delta-sigma modulators," in Circuits and Systems II: Express Briefs, IEEE Transactions on, vol. 54, no. 12, pp. 1125-1129, Dec. 2007.

[11] L. Breems, E. van der Zwan, and J. Huijsing, "Design for optimum performance-to-power ratio of a continuous-time $\Sigma \Delta$ modulator," in SolidState Circuits Conference, 1999. ESSCIRC '99. Proceedings of the 25th European, Sept. 1999, pp. 318-321.

[12] R. H. M. van Veldhoven and A. H. M. van Roermund, Robust Sigma Delta Converters And Their Application in Low-Power Highly-Digitized Flexible Receivers. Springer Netherlands, 2011.

[13] P. Gray, P. Hurst, S. Lewis, and R. Meyer, Analysis and Design of Analog Integrated Circuits. John Wiley \& Sons, 2001.

[14] M. Ortmanns, F. Gerfers, and Y. Manoli, "Compensation of finite gainbandwidth induced errors in continuous-time sigma-delta modulators," in 
Circuits and Systems I: Regular Papers, IEEE Transactions on, vol. 51, no. 6, pp. 1088-1099, June 2004.

[15] J. Kauffman, P. Witte, J. Becker, and M. Ortmanns, "An 8mW 50MS/s $\mathrm{CT} \Delta \Sigma$ modulator with $81 \mathrm{~dB}$ SFDR and digital background DAC linearization," in Solid-State Circuits Conference Digest of Technical Papers (ISSCC), 2011 IEEE International, Feb. 2011, pp. 472-474.

[16] R. van de Plassche, CMOS Integrated Analog-to-Digital and Digital-toAnalog Converters. Dordrecht, the Netherlands: Kluwer Academic Publishers, 2003.

[17] P. M. Figueiredo, "Comparator metastability in the presence of noise," in Circuits and Systems I: Regular Papers, IEEE Transactions on, vol. PP, no. 99, pp. 1-14, 2012.

[18] P. Nuzzo, F. De Bernardinis, P. Terreni, and G. Van der Plas, "Noise analysis of regenerative comparators for reconfigurable ADC architectures," in Circuits and Systems I: Regular Papers, IEEE Transactions on, vol. 55, no. 6, pp. 1441-1454, July 2008.

[19] J. A. Cherry and W. M. Snelgrove, Continuous-Time Delta-Sigma Modulators for High-Speed A/D Conversion: Theory, Practice and Fundamental Performance Limits. Norwell, MA, USA: Kluwer Academic Publishers, 2000.

[20] J. Silva et al., "Digital techniques for improved $\Delta \Sigma$ data conversion," in Custom Integrated Circuits Conference, 2002. Proceedings of the IEEE 2002, 2002, pp. 183-190.

[21] R. Baird and T. Fiez, "Linearity enhancement of multibit $\Delta \Sigma \mathrm{A} / \mathrm{D}$ and D/A converters using data weighted averaging," in Circuits and Systems II: Analog and Digital Signal Processing, IEEE Transactions on, vol. 42, no. 12 , pp. $753-762$, Dec. 1995.

[22] L. Risbo et al., "Digital approaches to ISI-mitigation in high-resolution oversampled multi-level D/A converters," in Solid-State Circuits, IEEE Journal of, vol. 46, no. 12, pp. 2892-2903, Dec. 2011.

[23] R. van Veldhoven, P. Nuijten, and P. van Zeijl, "The effect of clock jitter on the DR of $\Sigma \Delta$ modulators," in Circuits and Systems, 2006. Proceedings. 2006 IEEE International Symposium on, May 2006, p. 4. 
[24] J. Cherry and W. Snelgrove, "Clock jitter and quantizer metastability in continuous-time delta-sigma modulators," in Circuits and Systems II: Analog and Digital Signal Processing, IEEE Transactions on, vol. 46, no. 6, pp. 661-676, June 1999.

[25] S. Yan and E. Sanchez-Sinencio, "A continuous-time sigma-delta modulator with $88-\mathrm{dB}$ dynamic range and 1.1-MHz signal bandwidth," in SolidState Circuits, IEEE Journal of, vol. 39, no. 1, pp. 75-86, Jan. 2004.

[26] E. van der Zwan and E. Dijkmans, "A 0.2-mW CMOS $\Sigma \Delta$ modulator for speech coding with $80 \mathrm{~dB}$ dynamic range," in Solid-State Circuits, IEEE Journal of, vol. 31, no. 12, pp. 1873-1880, Dec. 1996.

[27] S. Pavan, N. Krishnapura, R. Pandarinathan, and P. Sankar, "A power optimized continuous-time $\Delta \Sigma \mathrm{ADC}$ for audio applications," in Solid-State Circuits, IEEE Journal of, vol. 43, no. 2, pp. 351-360, Feb. 2008.

[28] N. Pavlovic and J. Bergervoet, "A 5.3GHz digital-to-time-converter-based fractional-N all-digital PLL," in Solid-State Circuits Conference Digest of Technical Papers (ISSCC), 2011 IEEE International, feb. 2011, pp. 54-56. 


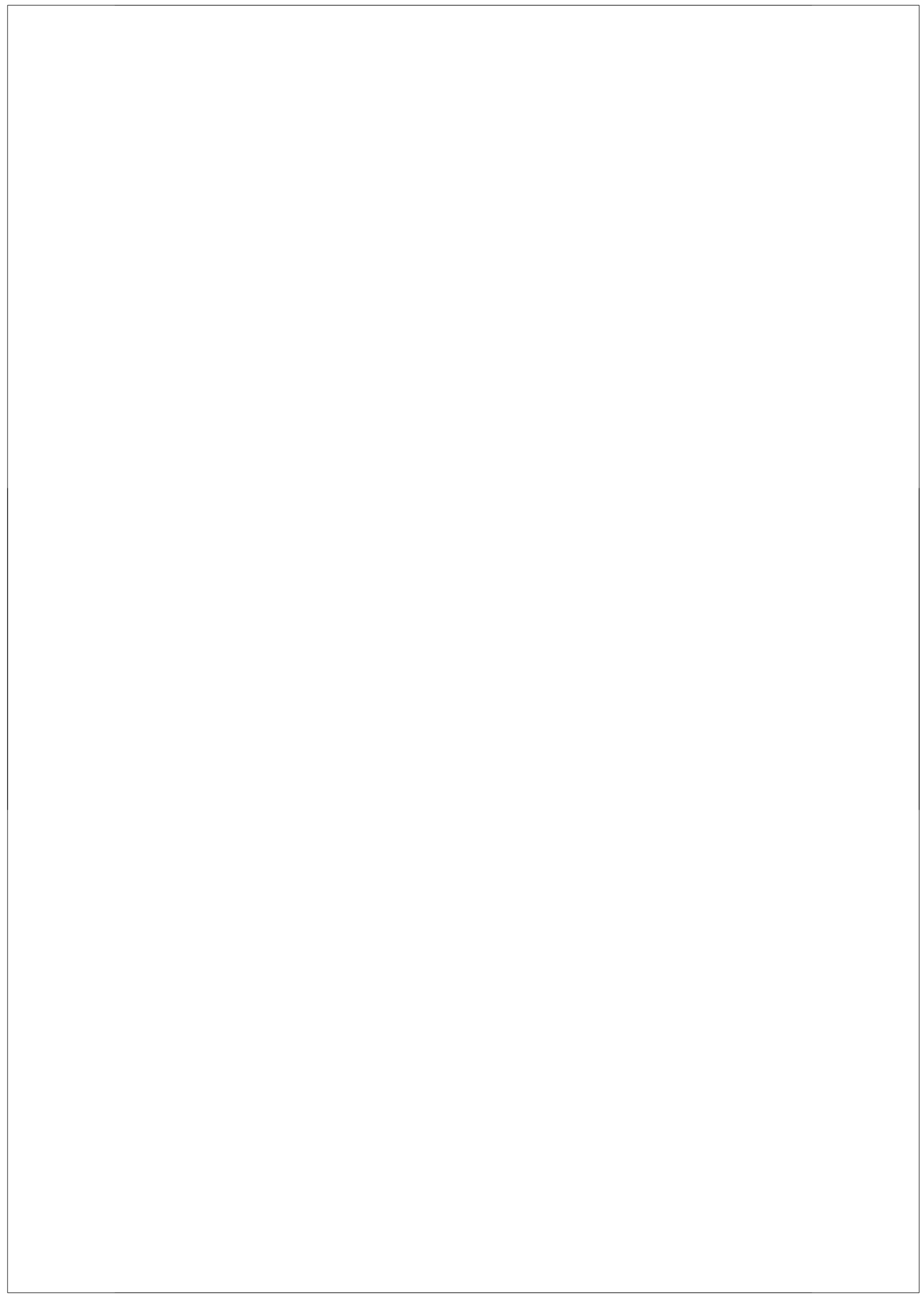




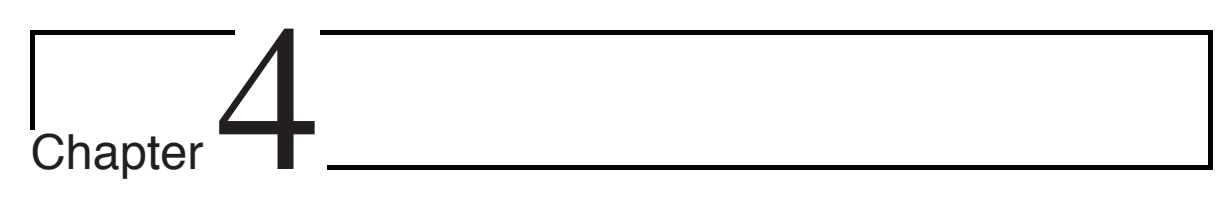

\section{A $4 \mathrm{GHz}$ Continuous-Time $\Delta \Sigma$ ADC}

\subsection{Introduction}

In this chapter, the implementation of a $4 \mathrm{GHz}$ continuous-time delta-sigma $(\mathrm{CT} \Delta \Sigma)$ ADC is presented that uses the high-speed filter topology proposed in Chapter 3. The ADC is fabricated in a $45 \mathrm{~nm}$ LP-CMOS with a supply voltage of $1.1 \mathrm{~V}$ with a target power dissipation of $400 \mathrm{~mW}$. The low supply voltage requires cascaded stages to make gain in blocks such as an OTA and a quantizer. The ADC employs a $3^{\text {rd }}$ order loop filter architecture with high-speed capacitive feedforward summation node. The ADC is sampled at $4 \mathrm{GHz}$ and uses a 4-bit quantizer which is designed for latency less than half a clock delay. The ADC employs a low noise current-steering feedback DAC (DAC1), which uses 1.8V supply to meet the noise and the matching requirements. Moreover, the excess loop delay (ELD) of the quantizer is compensated by a current steering DAC (DAC2) that is driven by a digital differentiator to implement a summation node at the output of the loop filter. The ADC achieves a 70dB dynamic range (DR) and $-74 \mathrm{dBFS}$ total harmonic distortion (THD) in a $125 \mathrm{MHz}$ bandwidth (BW) [1].

Section 4.2 discusses the implementation details of the building blocks of the ADC such as the loop filter, 4-bit high-speed quantizer and digital-toanalog converter (DAC). Section 4.3 describes the ADC's measurement setup and presents the measurement results which focus on the jitter performance of the $\mathrm{ADC}$ at the $4 \mathrm{GHz}$ sampling rate. 


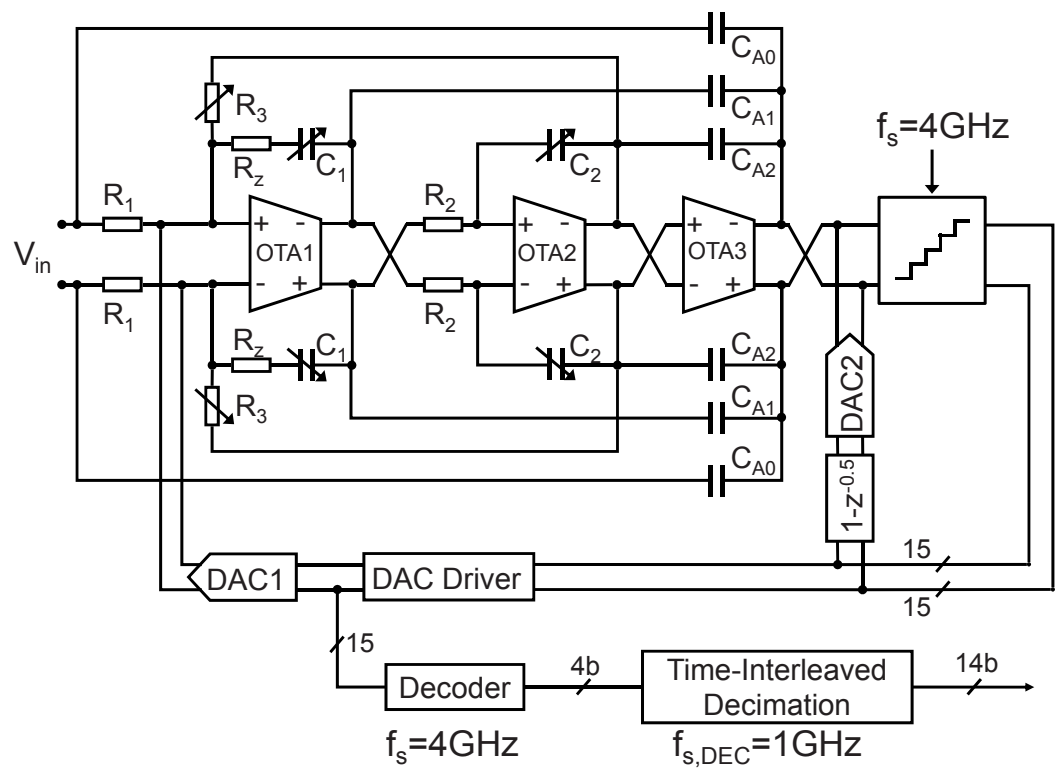

Figure 4.1 - The top-level architecture of the $3^{\text {rd }}$ order $\mathrm{CT} \Delta \Sigma \mathrm{ADC}$.

\subsection{Implementation Details}

\subsubsection{CT $\Delta \Sigma$ ADC Architecture}

Figure 4.1 shows the $3^{\text {rd }}$ order single-loop capacitive feedforward CT $\Delta \Sigma$ modulator in more detail. The first two integrators are implemented as RC integrators since these can operate at low supply voltages while providing the linearity required to achieve $-70 \mathrm{~dB}$ THD. Compared to open loop integrators such as $g_{m} C$ integrators, the feedback of the RC integrator linearizes the OTA. Moreover, the virtual ground node of the first integrator creates a summation node required for implementing the feedback of the modulator. To cancel the righthalf plane zero introduced by the limited $g_{m}$ of the first integrator, a resistor $\left(R_{z}=1 / g_{m}\right)$ in series with $C_{1}$ is employed [2]. The first and second operational transconductance amplifiers (OTAs) are implemented as two-stage amplifiers with feedforward frequency compensation [3]. To increase the gain in the band of interest, a resonator is implemented around the first two integrators by using a feedback resistor $\left(R_{3}\right)$. To compensate for RC spread, $C_{1}, C_{2}$ and $R_{3}$ can be individually calibrated via five-bit networks, for which the implemented 
tuning range covers $0.5-2 \times$ of the nominal $\mathrm{RC}$ time constant. This wide range of tuning also enables multi-mode operation of the ADC where the sampling frequency can be scaled down to $2 \mathrm{GHz}$, which is half of the original sampling rate $(4 \mathrm{GHz})$. The multi-mode operation of the $\mathrm{ADC}$ will be explained in more detail in Chapter 5. The third integrator is a $g_{m} C$ integrator since requirements on its linearity are relaxed by the gain of the first two integrators. As long as the last integrator's OTA does not clip, the loop filter achieves THD requirement listed in Table 3.2. In order to increase the input range of the third integrator, the third OTA is implemented as a resistively degenerated foldedcascode amplifier. Thanks to the high-speed capacitive feedforward loop filter architecture, the third OTA is not in the speed-critical path, which relaxes its BW requirements. As a result, its power dissipation is negligible compared to that of the first two OTAs. The feedforward capacitors $\left(C_{A 0}, C_{A 1}, C_{A 2}\right)$ are not made trimmable, since their relative matching can be made sufficiently accurate by layout. A further consideration is that the signal swing on the required selection switches could cause distortion via the signal-dependent ON resistances of the switches. The nominal bias current of the $g_{m} C$ integrator can also be varied $0.5-2 \times$ to calibrate its unity-gain frequency $\left(\omega_{3} \propto g_{m}\right)$.

The 15-bit thermometer code output of the 4-bit quantizer is connected through a DAC driver to the 4-bit DAC1. The DAC driver resamples the high speed data and generates digital copies for further processing. The ADC employs two 4-bit unary-weighted current-steering DACs. DAC1 is connected to the virtual ground node of the first integrator, where as DAC2 is directly connected to the capacitive summing node at the output of the loop filter. The ADC includes a thermometer-to-binary decoder, decimation filter and low voltage differential signaling (LVDS) buffers. The decoder demultiplexes the $4 \mathrm{GHz}$ data and converts the 15 -bit thermometer code into a $4 \times$ time-interleaved 4-bit binary code which is then decimated by an on-chip decimation filter.

\subsubsection{Quantizer Design and Timing Diagram of the Mod- ulator}

As shown in Fig. 4.2, the quantizer is a 4-bit flash converter. It consists of 15 unit elements whose reference voltages are generated from a 15 -tap resistive ladder. In order to meet the stability requirement of the modulator as discussed in Sections 3.1.3 and 2.2.3, the total delay around the quantizer and DAC1 must not exceed one clock period. Therefore, the latency of the quantizer must be less than half a sampling-clock period (125ps) to ensure loop stability. The combination of the 4-bit DAC1 and its driver (Fig. 4.1) must achieve similar delay while still meeting the linearity and noise requirements. Since DAC1 is 


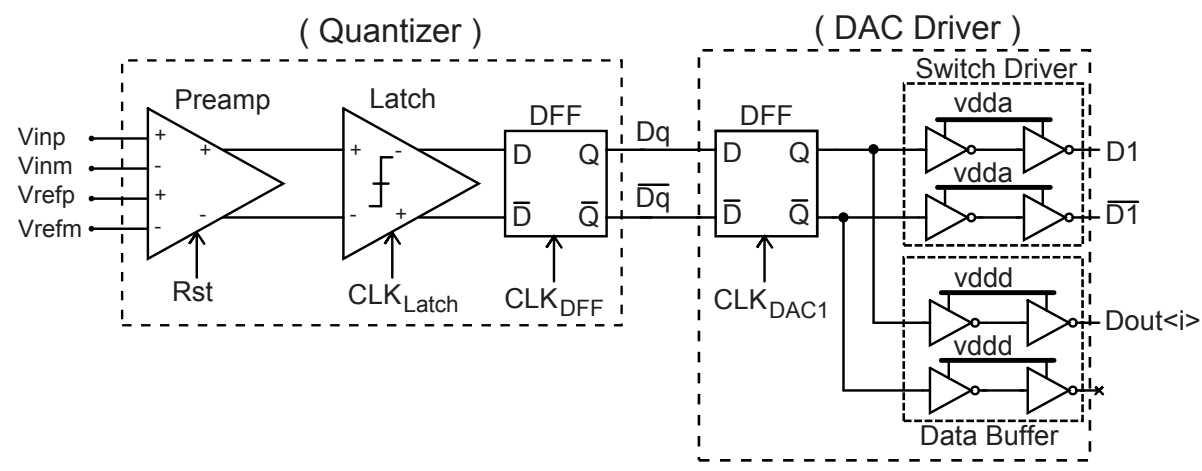

Figure 4.2 - Simplified schematic of a unit element of 4-bit quantizer and DAC driver.

connected to the input of the modulator, DAC1 is designed for good matching and low noise. Similar design requirements exists for the DAC1 driver because timing mismatch or clock jitter at the output transition of the DAC1 driver directly affects DAC1 output current. However, the DAC1 driver designed for good matching and low noise should not introduce latency that would lead to an unstable modulator. Lastly, the excess delay in the path around the 4-bit flash converter (through DAC2) must be less than half a clock period. Therefore, each slice of the quantizer drives a unit element of DAC2 to avoid the excess delay and power dissipation associated with re-clocking the data at $4 \mathrm{GHz}$. To meet these system-level requirements, the unit elements of the 4-bit quantizer and DAC1 driver are co-designed to minimize the total number of gates, and thus minimize the delay. Furthermore, the quantizer generates complementary digital outputs to drive DAC1 and DAC2 directly, while the high-speed digital traces are routed differentially to reduce the noise injected into the substrate.

To realize high-speed flash ADCs, several comparator stages can be pipelined, which increases the latency of the quantizer. In this design, however, the ADC must complete its operation in half a clock period, which severely limits the choice of architectures. Considering that at $4 \mathrm{GHz}$ the clock buffers will also consume considerable dynamic power, a three-stage comparator consisting of a preamplifier, a latch and a D-FF (Fig. 4.2) was chosen instead of a higher number of stages as a trade-off between the power consumption of the clock buffers and the power consumption of a unit slice of the quantizer.

The preamplifier is a resistively loaded NMOS pair with a reset switch connected across its output to enable fast overdrive recovery. The input pair is scaled for offset voltage and the preamplifier employs low-threshold transistors 


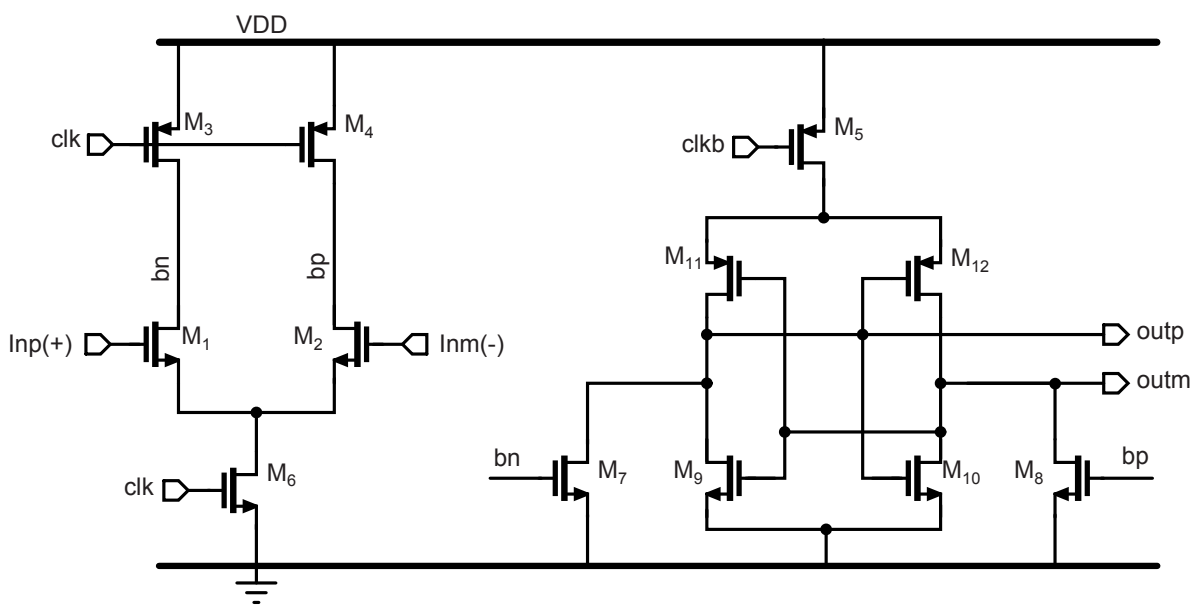

Figure 4.3 - Input stage of the D-FF.

to reduce the bias current required for the intended BW. The latch is realized as a differential pair that drives a cross-coupled latch. The D-FF consists of two stages: a double-tail sense amplifier [4] and a symmetrical slave latch (SL) [5]. The first stage of the D-FF is shown in Fig. 4.3. This architecture is suitable for low-voltage supplies since a maximum of three transistors are stacked between the supply rails. The second stage of the D-FF also uses a symmetrical SL, which ensures that each output of the D-FF has equal delay, making it possible to drive DAC2 directly and thus avoid the extra delay associated with reclocking the data. DAC1 driver uses the same D-FF architecture.

To reduce the kickback noise on the loop filter and reference ladder, the first two stages of the comparator (the preamplifier and the latch) are biased with a static current such that their input pairs do not switch. Only the charge injection of the reset switches is then present at the input of the comparator, although this is a common-mode effect. Moreover, the kickback noise of the $\mathrm{D}-\mathrm{FF}$ is suppressed by the gain of the first two stages of the quantizer. The $\mathrm{D}-\mathrm{FF}$ is also designed for minimal kickback noise. The first stage of the DFF (Fig. 4.3) consists of a dynamic input stage $\left(M_{1,2}\right)$ whose outputs (bn, bp) are connected to a cross-coupled inverter $\left(M_{9-11}\right)$ through $M_{7,8}$. Since the current of the latch can be optimized independently of the current of the input stage, the kickback noise caused by the switching of transistors $M_{1,2}$ can be minimized. Furthermore, $M_{7,8}$ isolates the input and output of the D-FF, which serves to further reduce the kickback noise. 


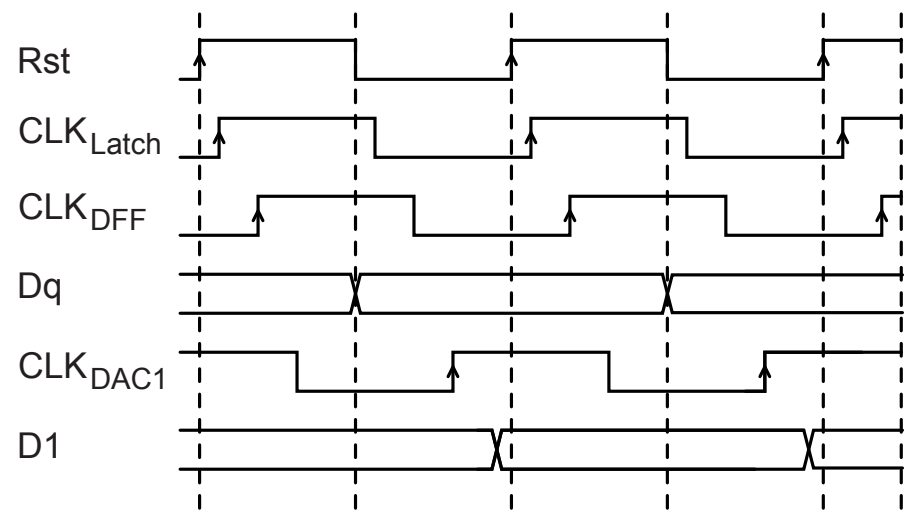

Figure 4.4 - Timing diagram of the $\mathrm{CT} \Delta \Sigma$ modulator.

The timing of the modulator is shown in Fig. 4.4. To ensure stability, the comparator outputs $(D q$ and $\overline{D q})$ must be valid after half a clock period, while the output of DAC1 driver ( $D 1$ and $\overline{D 1})$, which drives the unit current sources, must be valid within less than one clock period. In order to reduce the delay associated with the comparator, as well as the power in the clock buffers, a delayed-clocking scheme is adopted [6]. First, the preamplifier's $R s t^{1}$ switch is disabled and the preamplifier starts amplifying. After a short delay (less than half a clock period), during which the preamplifier's output settles to 4bit accuracy, $C L K_{\text {Latch }}$ is activated whereupon the signal is further amplified by the latch. Then $C L K_{D F F}$ is activated after which the D-FF finalizes the comparison and generates a valid digital representation of the decision. A unit element of the DAC driver is shown in Fig. 4.2. It consists of a D-FF, a switch driver, and a data buffer. The thermometer output of each quantizer is directly connected to each unit element, where it is re-clocked on the rising edge of $C L K_{D A C 1}$ (Fig. 4.4). The additional clocking of the data minimizes the jitter introduced by the D-FF's data-dependent delay and metastability.

\subsubsection{Feedback DACs}

DAC1 has the most stringent requirements in terms of linearity and noise, and it requires large devices to achieve the required matching. DAC2, which is connected to the output of the loop filter, has much more relaxed requirements, since its non-idealities are suppressed by the gain of the loop filter.

${ }^{1} R S T$ signal in Fig. 4.4 and $C L K_{Q}$ signal in Fig. $3.27 \mathrm{~b}$ represent the same signal. 


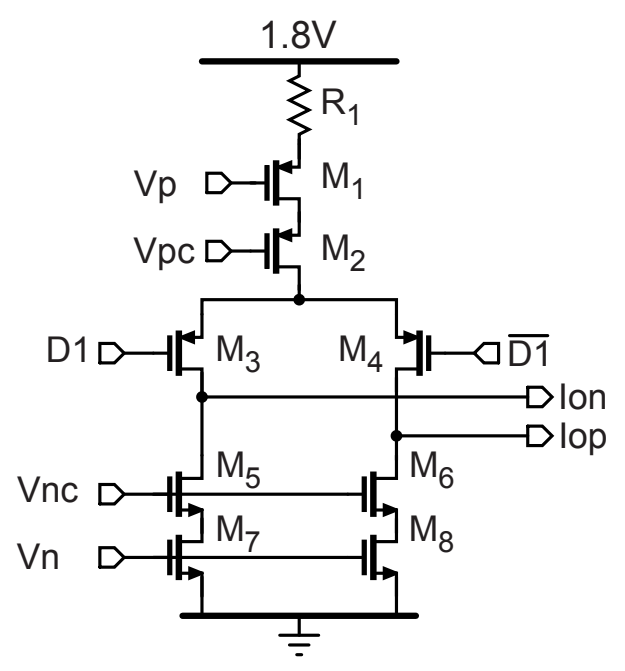

Figure 4.5 - Schematic of a unit element of DAC1.

DAC1 is a 4-bit current-steering DAC designed for 11-bit intrinsic matching. Achieving this with MOS current sources consumes too much area and results in poor high-frequency linearity. Increasing the gate overdrive voltage also does not help much, and so resistively degenerated current sources are used. One unit element of DAC1 is shown in Fig. 4.5. It consists of a resistively degenerated PMOS current source, which has better matching and lower noise than a MOS-only current source. By using a higher supply voltage for DAC1 $(1.8 \mathrm{~V}), \mathrm{R} 1$ can be made larger, effectively reducing the noise contribution of DAC1 and reducing the ADC's overall power consumption. Since the voltage drop on $\mathrm{R} 1$ is about $0.7 \mathrm{~V}, M_{1-8}$ can still be implemented using thin-oxide transistors. The D-FF and switch driver can then be optimized for the generation of the signals (with low crossover and steep edges) required to drive the PMOS switches $\left(M_{3,4}\right)$ of DAC1. At high sampling rates, the unequal rise and fall time of the output of DAC1 can cause inter-symbol interference (ISI) $[7,8]$. To minimize this, DAC1 employs a fully differential architecture [9]. Moreover, DAC1 driver's D-FF and switch drivers are dimensioned to achieve a signal-to-noise ratio (SNR) of better than $80 \mathrm{~dB}$ [7]. DAC1 is biased by lownoise on-chip circuitry, and for further noise suppression the bias voltage of the MOS current sources are filtered by an on-chip RC-filter. DAC1 does not use any calibration techniques such as data-weighted-averaging, or current-source calibration at the start-up. The linearity of DAC1 is thus limited by the device 


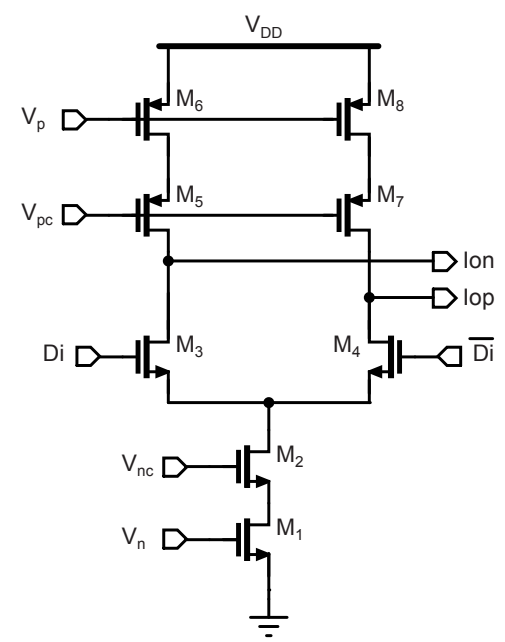

Figure 4.6 - Schematic of DAC2 current source.

matching.

DAC2 is a 4-bit current-steering DAC, and its errors are suppressed by the gain of the loop filter, and so it is designed for 9-bit intrinsic matching (Section 3.2.4). Figure 3.27a illustrates the block diagram of DAC2. To reduce the latency, the differentiation $\left(1-z^{-0.5}\right)$ and DAC functionality are integrated into each DAC2 unit. Each DAC2 unit has two current sources and a DFF. The detailed functionality and timing diagram of DAC2 are explained in Section 3.2.4. One unit element of DAC2 current source (C.S.) is shown in Fig. 4.6. It uses an NMOS current source $\left(M_{1}\right)$ which is cascoded with $M_{2}$ to improve its output impendence. The data switches use NMOS transistors $\left(M_{3,4}\right)$ with $40 \mathrm{~nm}$ channel length to reduce the loading to the quantizer.

\subsubsection{Operational Transconductance Amplifier}

As shown in Fig. 4.7, the first two integrators are implemented as a two-stage feedforward compensated amplifier [3]. Transistors $M_{1-8}$ form the amplifier's input stage, while transistors $M_{11,12}$ form its second stage. Transistors $M_{9,10}$ create a high-frequency feedforward path between the input and the output, thus stabilizing the amplifier. The output common-mode voltage of the first stage is sensed by poly resistors that control the gate voltage of transistors $M_{7,8}$. Similarly the output common-mode voltage of the second stage is controlled by an auxiliary common-mode amplifier which controls the bias voltage 


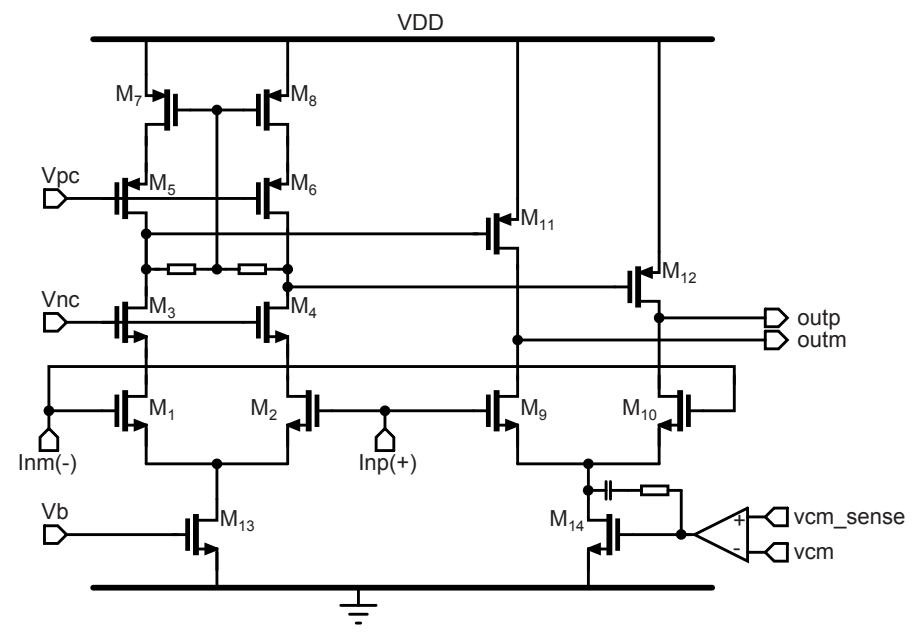

Figure 4.7 - Schematic of the operational transconductance amplifier.

of transistor $M_{14}$. The designed OTA achieves a $35 \mathrm{~dB}$ DC gain and an $8 \mathrm{GHz}$ unity-gain-bandwidth (UGBW), while consuming $23 \mathrm{~mA}$ from a $1.1 \mathrm{~V}$ supply. Since the OTA of the second integrator requires less bandwidth, its current is scaled down by a factor of two. The third OTA is implemented as a resistively degenerated folded-cascode amplifier in order to increase its linear input range. Thanks to the high-speed capacitive feedforward loop filter architecture, the third OTA is not in the speed-critical path, which relaxes its BW requirements. As a result, its power dissipation is negligible compared to that of the first two OTAs.

\subsubsection{Decimation Filter}

Figure 4.8 illustrates the block diagram of the thermometer-to-binary decoder and decimation filter. The decimation filter is included on the chip to relax the task of capturing the data and designing the test PCB. Moreover, the decoder and decimation filter act as a digital aggressor when in close proximity to the ADC. Therefore, the robustness of the ADC's performance to substrate noise injected by the digital circuitry can be evaluated. The 15-bit thermometer output of the modulator is clocked at $4 \mathrm{GHz}$. Since the digital cells of the standard digital library could only be verified up to $1.2 \mathrm{GHz}$, the data is first demultiplexed by a custom thermometer-to-binary decoder which generates $4 \times$ time-interleaved 4-bit binary data with a sampling frequency of $1 \mathrm{GHz}$. The 


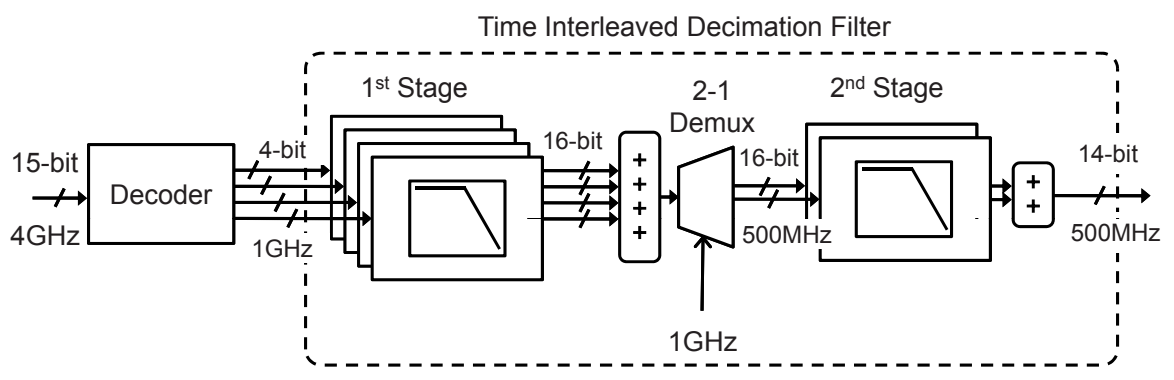

Figure 4.8 - Block diagram of the implemented decimation filter.

two-stage polyphase decimation filter sampled at $1 \mathrm{GHz}$ and $500 \mathrm{MHz}$ respectively, generates 14 -bit decimated outputs at $500 \mathrm{MHz}$ so that the quantization noise spectrum just outside the $125 \mathrm{MHz}$ signal $\mathrm{BW}$ can also be measured. The decimated outputs are then converted to LVDS signals on the chip and transmitted to LVDS repeaters on the measurement PCB.

\subsection{Experimental Results}

\subsubsection{Measurement Setup}

The measurement setup used to evaluate the ADC is shown in Fig. 4.9. A signal source (Rohde \& Schwarz SMA100A) drives a programmable $5^{\text {th }}$ order bandpass filter which attenuates its harmonics and the noise below 100dBc. The resulting single-ended signal is converted into a differential signal by a balun and fed to the ADC. The ADC's clock signal is generated by another signal source (Rohde \& Schwarz SMIQ-06B) which outputs a $4 \mathrm{GHz}$ sinewave with $6 \mathrm{dBm}$ of output power. The integrated jitter of the clock signal is $240 \mathrm{fsec}$ root-mean-square (RMS) in a $1 \mathrm{kHz}$ to $2 \mathrm{GHz} \mathrm{BW}$. The clock signal is converted into a differential signal $(C L K, \overline{C L K})$ by a $180^{\circ}$-hybrid and then AC-coupled to the ADC. This divides it by four and outputs the result to enable data capture and synchronization. A pulse generator (Agilent $81134 \mathrm{~A}$ ) is synchronized to $C L K_{O U T}$ and outputs a conditioned CLK to a high-speed FPGA (Altera Stratix III) which captures the data. LVDS repeaters on the test PCB buffer the decimated 14-bit output of the ADC and isolate it from the digital noise associated with the FPGA. The captured data is then downloaded to a PC for post processing in MATLAB. At GHz sampling speeds, capturing errors 


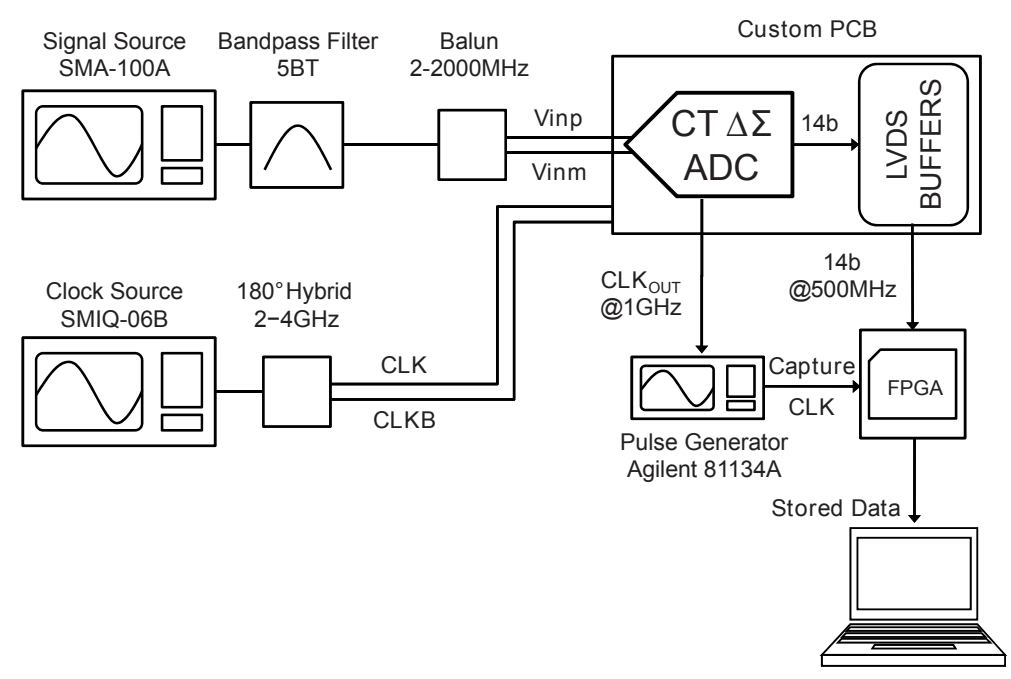

Figure 4.9 - Measurement setup of the CT $\Delta \Sigma$ ADC.

can degrade the measurement results, therefore a double sampling scheme is adapted to capture data. The data is sampled twice by the FPGA and so every consecutively captured sample will have the same value if the measurement setup has the correct timing and synchronization. This sampling scheme provides a $1^{\text {st }}$ order confirmation that no capturing errors have occurred.

\subsubsection{Measurement Results}

A chip photo of the fabricated ADC in 45nm baseline LP-CMOS is shown in Fig. 4.10. The ADC has an active area of $0.9 \mathrm{~mm}^{2}$. The modulator occupies $0.675 \mathrm{~mm}^{2}$, whereas the clock buffers and decimation filter occupy $0.225 \mathrm{~mm}^{2}$. The ADC including the decimation filter dissipates $256 \mathrm{~mW}$ from a $1.1 \mathrm{~V}$ supply and $3.2 \mathrm{~mW}$ from a $1.8 \mathrm{~V}$ supply. To reduce interconnect resistances and capacitances, the high speed blocks are placed very close to each other. For example, DAC2 with its multi-bit differentiator is located just after the 4-bit quantizer. DAC1 is positioned very close to the input of the loop filter, so as to minimize the parasitics at the virtual ground of the first integrator. At the system level, the additional delay due to the long interconnect lines between the 4-bit quantizer and DAC1 is compensated for by allocating a half clock cycle to the sum of its settling time and the interconnect delay. Both the clock 


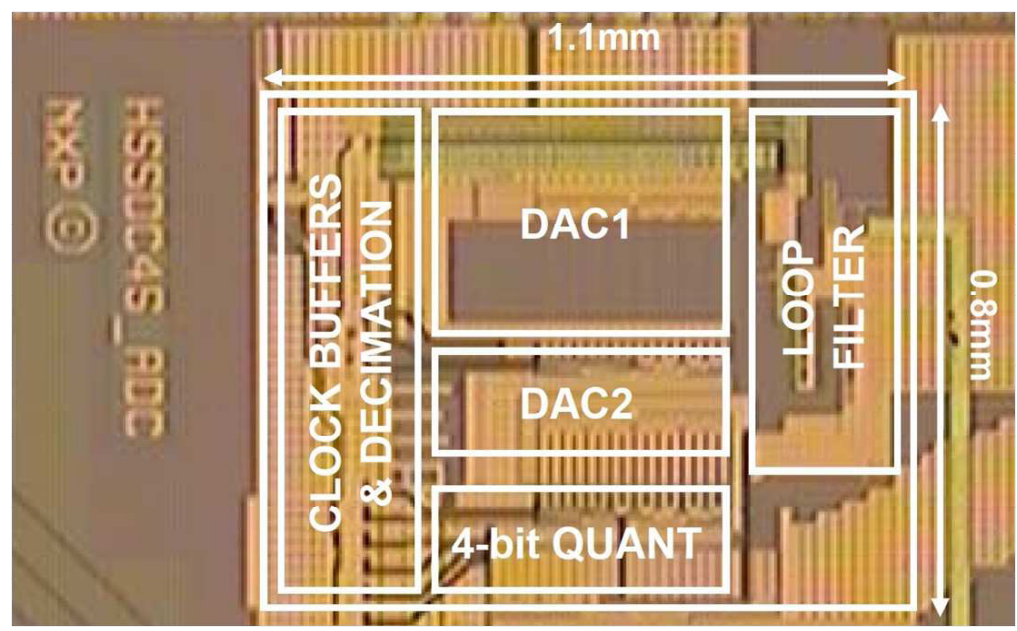

Figure 4.10 - Chip Micrograph.

buffers and digital circuits, such as the decoder and the decimation filter, are positioned close to the clocked circuits. Moreover, identical supply routing is used for DAC1, DAC2, and the quantizer to ensure that each unit element experiences the same $\mathrm{I} \times \mathrm{R}$ drop in its supply.

Figure 4.11 shows an FFT of the measured-decimated output of the $\Delta \Sigma$ ADC with no input signal. The ADC's noise floor ${ }^{2}$ is flat in the signal BW of $125 \mathrm{MHz}$ and rises slightly at higher frequencies due to the presence of out-ofband quantization noise. To measure the ADC's distortion, sinusoidal input signals with a maximum input voltage of $2.0-\mathrm{V}_{\mathrm{p}-\mathrm{p}}$ differential were supplied to the ADC. The decimated output for a $41 \mathrm{MHz}$ input signal at $-0.5 \mathrm{dBFS}$ has been captured in real-time; its FFT is shown in Fig. 4.11. The THD is -74 dBFS. As shown in Fig. 4.12, the ADC achieves a $70 \mathrm{~dB}$ DR in a $125 \mathrm{MHz}$ BW. The peak SNR/SNDR are $65.5 / 65 \mathrm{~dB}$ at $-0.5 \mathrm{dBFS}$ input respectively. For large signals $(-10 \mathrm{dBFS} \sim-0.5 \mathrm{dBFS})$, the residual non-linearity of DAC1 causes harmonic components and quantization errors to fold into the signal band, thus increasing the in-band noise.

Figure 4.13 shows the ADC's measured intermodulation performance for $93 \mathrm{MHz}$ and $95 \mathrm{MHz}$ input signals at $-7.2 \mathrm{dBFS}$. This choice of input frequency was determined by the bandpass filters available in the measurement setup. The $2^{\text {nd }}$ order intermodulation distortion $\left(\mathrm{IM}_{2}\right)$ and the $3^{\text {rd }}$ order intermodulation

\footnotetext{
${ }^{2}$ In Figs. 4.11-4.13, the noise floor is the average of four measurements.
} 


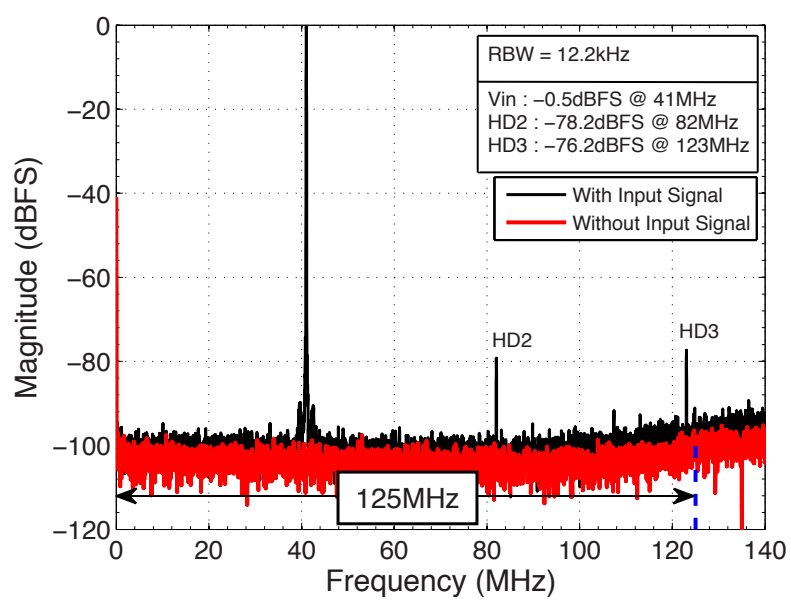

Figure 4.11 - An FFT of measured decimated output for an input signal of $-0.5 \mathrm{dBFS}$ at $41 \mathrm{MHz}$.

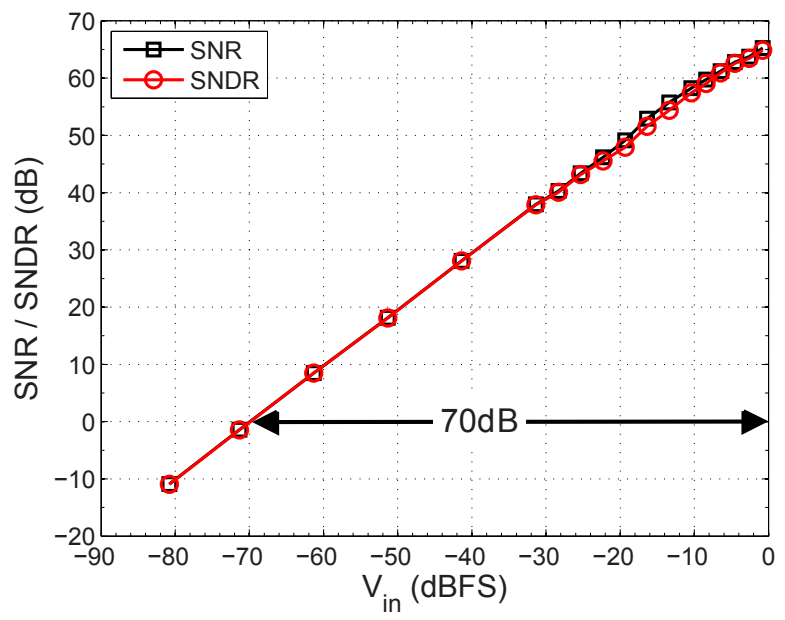

Figure 4.12 - Measured SNR and SNDR vs. input signal level $\left(f_{\text {in }}=41 \mathrm{MHz}\right)$. 


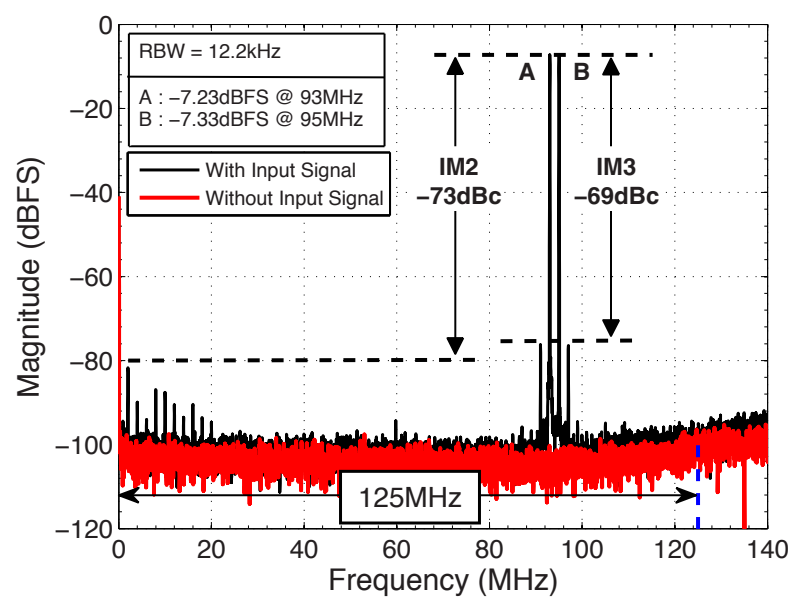

Figure 4.13 - An FFT of measured decimated output for a two-tone input signal of $-7.3 \mathrm{dBFS}$ at $93 \mathrm{MHz}$ and $95 \mathrm{MHz}$.

distortion $\left(\mathrm{IM}_{3}\right)$ are $-73 \mathrm{dBc}$ and $-69 \mathrm{dBc}$, respectively. The measured linearity of the $\mathrm{ADC}$ is limited by the mismatch of $\mathrm{DAC} 1$ unit elements.

The jitter performance of a $\mathrm{CT} \Delta \Sigma \mathrm{ADC}$ is commonly analyzed by assuming a clock source with white noise jitter. However, to generate $\mathrm{GHz}$ sampling frequencies in practice, an on-chip clock source such as a PLL is required. This will multiply an input reference clock and generate the ADC's sampling clock $\left(f_{s}\right)$. As is typical in a PLL output spectrum, the clock would then have spurious tones located at $\left(f_{s} \pm f_{\text {offset }}\right)$. In multi-channel applications, these spurious tones can demodulate an adjacent channel or an interferer into the signal band and thus degrade the sensitivity of the receiver. For an input signal located at $f_{i n}$, the amplitude of in-band jitter tones at the ADC's output can be expressed as [10]:

$$
\mathrm{JT}_{f_{i n} \pm f_{\text {offset }}}=\mathrm{ST} \times \frac{f_{\text {in }}}{f_{s}}[d B c]
$$

where ST is the power ratio of a spurious tone relative to the carrier. Since the implemented ADC does not have a PLL, an external clock signal ${ }^{3}$ was used to generate a spurious tone located at $f_{\text {offset }}=10 \mathrm{MHz}$ with $-32.4 \mathrm{dBc}$

${ }^{3} \mathrm{~A}$ signal source generates a sinewave that is fed to a pattern generator (Agilent J-BERT N4903B) which divides the input clock signal by two and generates a $4 \mathrm{GHz}$ clock signal with $6 \mathrm{dBm}$ output power. 
of power, as shown in Fig. 4.14a. To measure the in-band jitter tones, a $105 \mathrm{MHz}$ input signal at $-1 \mathrm{dBFS}$ is applied to the ADC input; the resulting jitter tones are shown in Fig. 4.14b. The jitter tones are attenuated by $10 \cdot \log _{10}(105 \mathrm{MHz} / 4 \mathrm{GHz})=31.6 \mathrm{dBc}$ and the resulting tones located at $f_{\text {in }} \pm f_{\text {offset }}$ have amplitudes of $-63.8 \mathrm{dBc}$ and $-63.9 \mathrm{dBc}$, respectively, which agrees with (4.1).

However, the DR of the ADC is reduced due to the white noise jitter. To measure the effect of white noise jitter, a BW-limited white noise jitter is introduced by using a pattern generator. The signal-to-jitter-noise-ratio (SJNR) due to the demodulation of the out-of-band quantization noise can be expressed as:

$$
\begin{aligned}
\operatorname{SJNR}_{J Q}= & -10 \cdot \log _{10}(P N D) \\
& -10 \cdot \log _{10}(B W) \\
& +10 \cdot \log _{10}\left[(N-1)^{2}\right] \\
& -10 \cdot \log _{10}\left[\left(\frac{N-1}{0.7+N-2}\right)^{2}\right]+6,
\end{aligned}
$$

where PND is the average phase noise density per $\mathrm{Hz}, \mathrm{N}$ is the number of quantizer levels, and BW is the signal BW [11]. In (4.2) it is assumed that all the quantization noise is located at $0.5 \times f_{s}$, which results in a lower SJNR for a given white noise jitter. In Fig. $4.15 \mathrm{a}$, the phase noise spectrum of the clock generator around the carrier without additional white noise is shown (clock source). The ADC normally achieves a $70 \mathrm{~dB}$ DR, but when $-34.5 \mathrm{dBc}(1.05 \mathrm{psec}$ RMS) white noise is applied to the clock (test clock $^{4}$ in Fig. 4.15a), its DR degrades to $69 \mathrm{~dB}$, as shown in Fig. 4.15b. By using (4.2), the expected SJNR ${ }^{5}$ is $75.2 \mathrm{~dB}$, which reduces the DR by $1 \mathrm{~dB}$.

However, in the presence of a large input signal, the white noise jitter in Fig. $4.15 \mathrm{a}$ is present around the input signal and degrades the SJNR significantly, as shown in Fig. 4.15b. The SNR degrades from $65 \mathrm{~dB}$ to $61 \mathrm{~dB}$ as expected from (4.1). Therefore, in the presence of a large input signal in high-speed,

\footnotetext{
${ }^{4}$ While generating white noise jitter, the test clock generates spurious tones located up to $2 \mathrm{MHz}$ offset from the carrier.

${ }^{5}$ The measured integrated phase noise is $-34.5 \mathrm{dBc}$ in a $100 \mathrm{MHz} \mathrm{BW}$ from the carrier frequency $(\mathrm{PND}=-114 \mathrm{dBc} / \mathrm{Hz})$. For the frequencies between $100 \mathrm{MHz}$ and $500 \mathrm{MHz}$ offset from the carrier, PND stays at $-114 \mathrm{dBc} / \mathrm{Hz}$, and for frequencies higher than $500 \mathrm{MHz}, \mathrm{PND}$ rolls off to $-138 \mathrm{dBc} / \mathrm{Hz}$. However, since the quantization noise is low enough for frequencies between $100 \mathrm{MHz}$ and $500 \mathrm{MHz}$, the convolution of white noise jitter and quantization noise can be neglected. Therefore, the phase noise density can be assumed to be at $-138 \mathrm{dBc} / \mathrm{Hz}$. The total integrated phase noise (in the band of $1 \mathrm{kHz}-2 \mathrm{GHz}$ ) is $-34.2 \mathrm{dBc}$. The $\mathrm{PND}^{\mathrm{dBc} / \mathrm{Hz}}$ is $-34.2 \mathrm{dBc}-10 \log _{10}\left(0.5 \times \mathrm{f}_{\mathrm{S}}\right)=-127.2 \mathrm{dBc}$, and by using $(4.2)$, the expected SJNR is $75.2 \mathrm{~dB}$.
} 


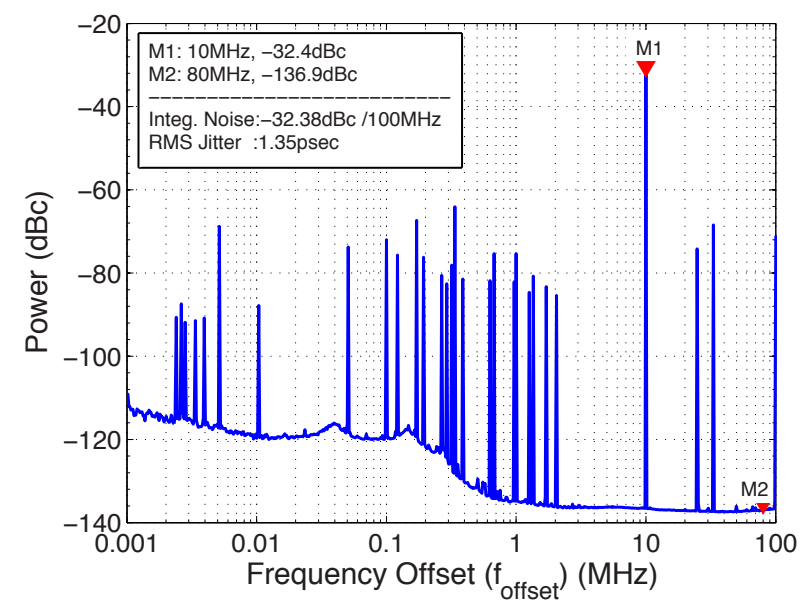

(a)

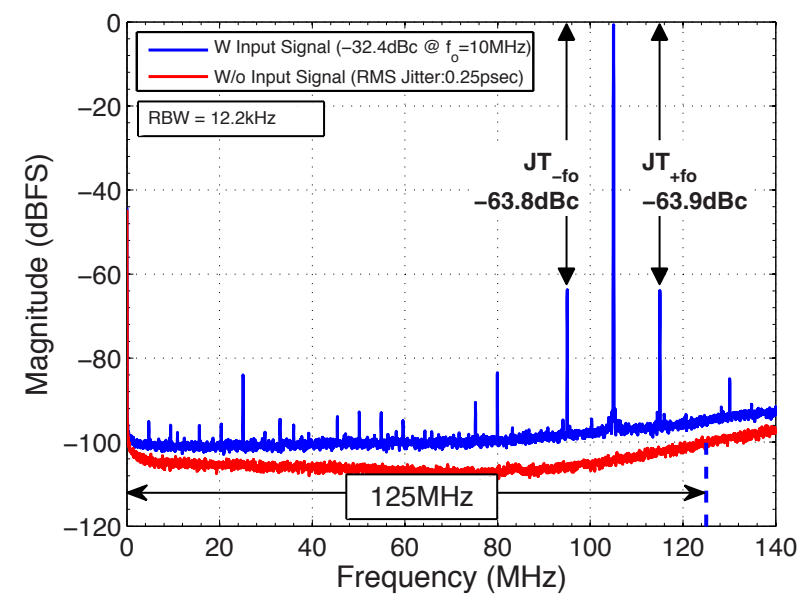

(b)

Figure 4.14 - The measured phase noise of the clock source for a clock tone introduced at $f_{c}+10 \mathrm{MHz}$ with $-32 \mathrm{dBc}$ power (a), and the measured output spectrum of the CT $\Delta \Sigma$ ADC for an input signal of $105 \mathrm{MHz}$ at $-0.5 \mathrm{dBFS}$. 
wideband $\mathrm{CT} \Delta \Sigma$ ADCs, the spectral shape of jitter noise limits the achievable SNR and DR.

Spurious tones are present at $25 \mathrm{MHz}, 80 \mathrm{MHz}$, and $130 \mathrm{MHz}$ in both Figs. $4.14 \mathrm{~b}$ and $4.15 \mathrm{~b}$. However, the clock spectrum in Fig. 4.15a does not have any spurious tones above $2 \mathrm{MHz}$. Thus these high frequency spurious tones are not due to clock spurs. Since the decimation filter is effectively running at $500 \mathrm{MHz}$ and it has a limited out-of-band attenuation, aliasing in the decimation filter might cause these tones to occur. For example, the higher-order distortion tones of the modulator $\left(4^{\text {th }}, 5^{\text {th }}, 6^{\text {th }}, \ldots\right)$ can mix down with the clock of the decimation filter.

The 16 samples that have been measured showed similar performances. Table 4.1 summarizes the performance of a typical ADC sample. Compared to the CMOS $\Delta \Sigma$ ADCs, the proposed ADC achieves a $5 \mathrm{x}$ larger $\mathrm{BW}$ with a similar dynamic range. When compared to non-CMOS $\Delta \Sigma$ ADCs, it achieves a $125 \mathrm{MHz}$ BW with $10 \mathrm{~dB}$ more of $\mathrm{DR}$ with both a lower supply voltage and lower sampling frequency $\left(f_{s}=4 \mathrm{GHz}\right)$. The $\mathrm{ADC}$ has a figure of merit (FoM) of $0.71 \mathrm{pJ} /$ conv.-step, where the FoM is defined as:

$$
\mathrm{FoM}=\frac{\text { Power }}{2 \times \mathrm{BW} \times 2^{(\mathrm{SNDR}-1.76) / 6.02}} .
$$

In the FoM calculation, the power consumption of the modulator, clock buffers, decoder and decimation filter are included. The proposed ADC's FoM is more than $10 \times$ better than CMOS $\Delta \Sigma$ ADCs. It owes its power efficiency to both its loop-filter architecture, which obviates the need for a power-hungry active summation node, and to the low power consumption of the digital circuitry in $\mathrm{nm}$-CMOS. Since the switching speed of a transistor increases by $1.6 \mathrm{x}$ from $90 \mathrm{~nm}$ CMOS to $45 \mathrm{~nm}$ LP-CMOS, the rest of the improvement in the signal BW is achieved thanks to the use of a high-speed capacitive-feedforward loop-filter architecture, and a low-latency 4-bit quantizer and DAC. Compared to the Nyquist ADC, the proposed ADC achieves a similar BW but one-bit less dynamic range. Since the DR of the proposed ADC is thermal-noise limited, it can be improved by reducing its effective input-referred noise resistance. This will be at the expense of increased power consumption in the first integrator, which, however, contributes only $10 \%$ of the ADC's total power dissipation. The proposed ADC has a better FoM than the Nyquist ADC [12] which implies that $\Delta \Sigma$ ADCs can be a power-efficient alternative for applications which require a high dynamic range and wide BWs. Lastly, the active area of the proposed ADC is less than $1 \mathrm{~mm}^{2}$, which facilitates low-cost integration. 


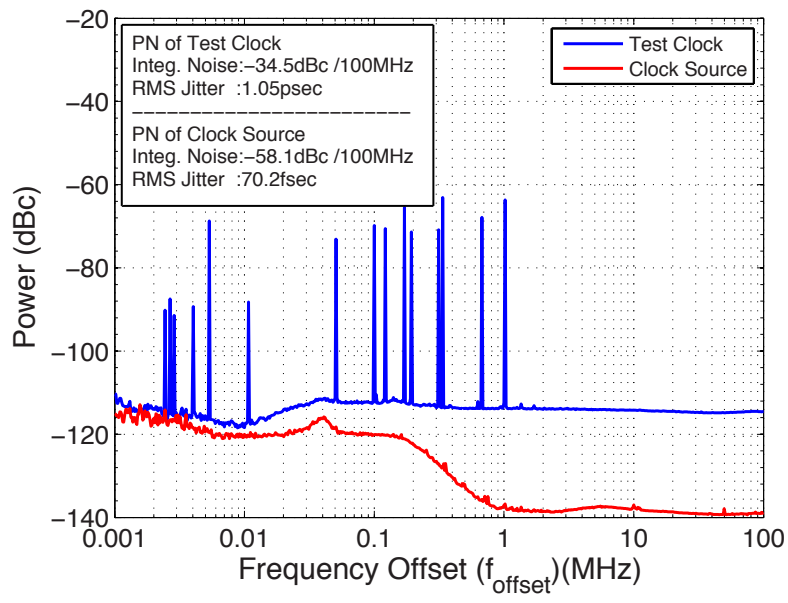

(a)

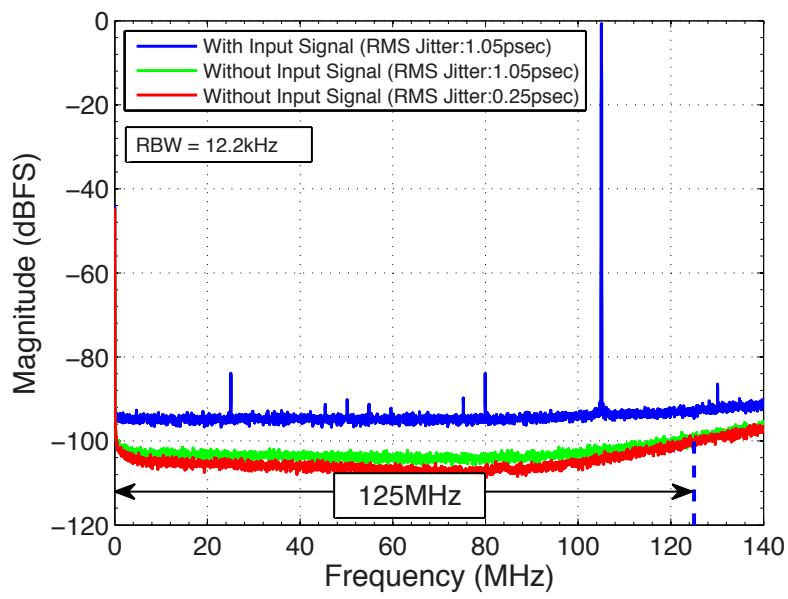

(b)

Figure 4.15 - Measured phase noise of the clock source and the test clock with wideband white noise (a), and the measured output spectrum of the CT $\Delta \Sigma$ $\mathrm{ADC}$ for an input signal of $105 \mathrm{MHz}$ at $-0.5 \mathrm{dBFS}$. 
Experimental Results

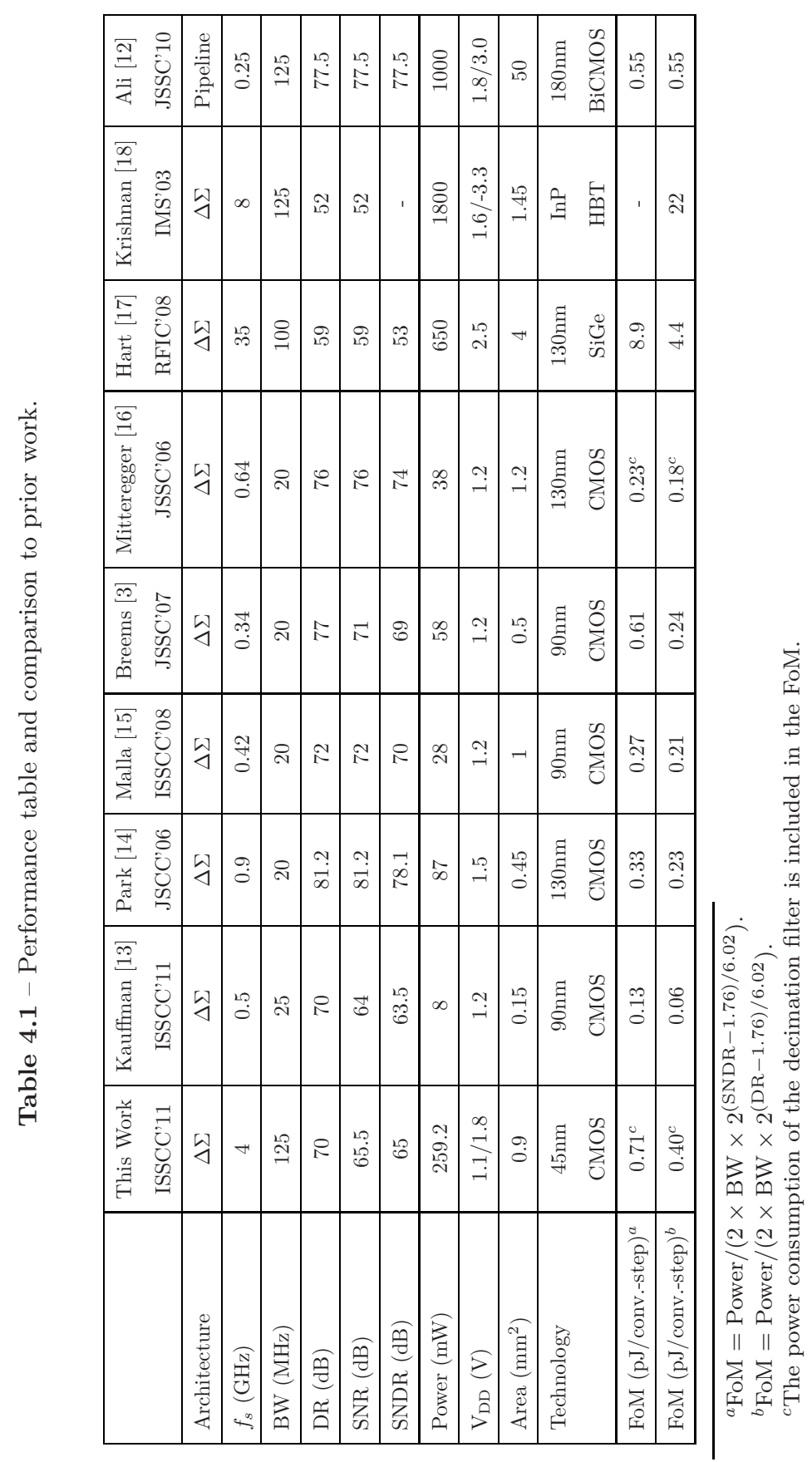




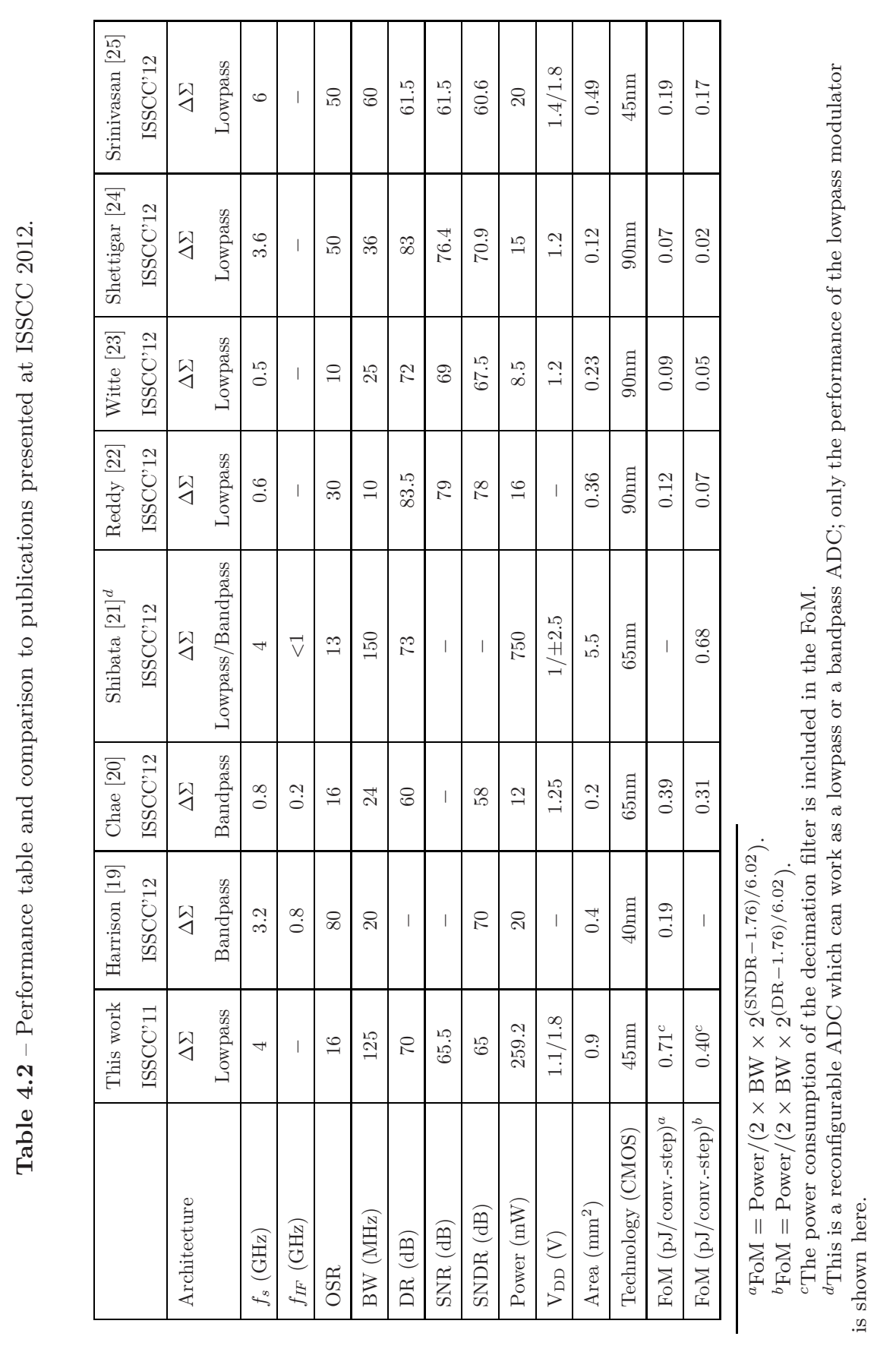


Recently, $\mathrm{CT} \Delta \Sigma$ ADCs implemented in nm-CMOS have gained popularity. Table 4.2 summarizes the performance of these analog-to-digital converters (ADCs). Compared to the non-CMOS implementations indicated in Table 4.1, CMOS implementations achieve better power efficiency. This can be attributed to the use of nm-CMOS in which dynamic power scales with smaller feature sizes. However, as can be seen in Table 4.2, the FoM of the ADCs implemented in nm-CMOS differs significantly. In fact, the ADC with the lowest active chip area for every process node, has the best power efficiency. Therefore, the area of the chip is likely to be a sign of power efficiency. In addition for modulators with a sampling speed $\left(f_{s}\right)$ higher than $1 \mathrm{GHz}$, the same trend is observed even though the ADCs are implemented in technologies with larger feature sizes. In other words, to improve the power efficiency, the modulator should be designed to be as small as possible. This approach can help us to reduce the power required to distribute clock between the circuit blocks such as the quantizer, DAC and clock buffers. On the other hand, the ADCs which deliver the $\mathrm{BW}>100 \mathrm{MHz}$ suffer from a lower FoM $[1,21]$ compared to the modulators with $20 \mathrm{MHz} \mathrm{BW}$. As the scaling of nm-CMOS continues, the BW of a CT $\Delta \Sigma$ $\mathrm{ADC}$ is also expected to scale with improvements in transistor switching speed. For example, $20 \%$ more signal BW can be achieved in 28nm LP-CMOS.

\subsection{Conclusions}

The work presented in this chapter demonstrates the implementation of a multibit $\mathrm{GHz} \mathrm{CT} \Delta \Sigma \mathrm{ADC}$ that achieves a $70 \mathrm{~dB}$ dynamic range in a $125 \mathrm{MHz}$ signal BW. Without any calibration, the ADC achieves $-74 \mathrm{~dB}$ THD in a $125 \mathrm{MHz}$ BW with a FoM of $0.71 \mathrm{pJ} /$ conv.-step, while drawing only $256 \mathrm{~mW}$ from a $1.1 \mathrm{~V}$ supply and $3.2 \mathrm{~mW}$ from a $1.8 \mathrm{~V}$ supply. This performance is achieved thanks to the use of a high-speed capacitive-feedforward loop filter architecture, and a low-latency 4-bit quantizer and DAC. Furthermore, its resistive input makes it easier to drive than Nyquist ADCs with switched-capacitor inputs. The result is an ADC design whose performance enlarges the application domain of $\Delta \Sigma$ ADCs by an order of magnitude.

\section{References}

[1] M. Bolatkale, L. Breems, R. Rutten, and K. Makinwa, "A 4GHz CT $\Delta \Sigma$ ADC with 70dB DR and -74dBFS THD in 125MHz BW ," in Solid-State Circuits Conference, 2011. ISSCC 2011. Digest of Technical Papers. IEEE International, Feb. 2011, pp. 470-472. 
[2] P. Gray, P. Hurst, S. Lewis, and R. Meyer, Analysis and Design of Analog Integrated Circuits. John Wiley \& Sons, 2001.

[3] L. Breems, R. Rutten, R. van Veldhoven, and G. van der Weide, "A 56 $\mathrm{mW}$ continuous-time quadrature cascaded $\Sigma \Delta$ modulator with $77 \mathrm{~dB}$ DR in a near zero-IF $20 \mathrm{MHz}$ band," in Solid-State Circuits, IEEE Journal of, vol. 42, no. 12, pp. 2696-2705, Dec. 2007.

[4] D. Schinkel et al., "A double-tail latch-type voltage sense amplifier with 18ps setup+hold time," in Solid-State Circuits Conference, 200\%. ISSCC 200\%. Digest of Technical Papers. IEEE International, Feb. 2007, pp. 314605.

[5] B. Nikolic et al., "Improved sense-amplifier-based flip-flop: design and measurements," in Solid-State Circuits, IEEE Journal of, vol. 35, no. 6, pp. 876-884, June 2000.

[6] M. Choi, J. Lee, J. Lee, and H. Son, "A 6-bit 5-GSample/s Nyquist A/D converter in 65nm CMOS," in VLSI Circuits, 2008 IEEE Symposium on, June 2008, pp. 16-17.

[7] R. W. Adams, "Design and implementation of an audio 18-bit analog-todigital converter using oversampling techniques," in J. Audio Eng. Soc, vol. 34, no. 3, pp. 153-166, 1986.

[8] E. van der Zwan and E. Dijkmans, "A 0.2-mW CMOS $\Sigma \Delta$ modulator for speech coding with $80 \mathrm{~dB}$ dynamic range," in Solid-State Circuits, IEEE Journal of, vol. 31, no. 12, pp. 1873-1880, Dec. 1996.

[9] J. Jensen, G. Raghavan, A. Cosand, and R. Walden, "A 3.2-GHz secondorder delta-sigma modulator implemented in InP HBT technology," in Solid-State Circuits, IEEE Journal of, vol. 30, no. 10, pp. 1119-1127, Oct. 1995.

[10] R. van Veldhoven, P. Nuijten, and P. van Zeijl, "The effect of clock jitter on the DR of $\Sigma \Delta$ modulators," in Circuits and Systems, 2006. Proceedings. 2006 IEEE International Symposium on, May 2006, p. 4.

[11] R. H. M. van Veldhoven and A. H. M. van Roermund, Robust Sigma Delta Converters And Their Application in Low-Power Highly-Digitized Flexible Receivers. Springer Netherlands, 2011.

[12] A. Ali et al., "A 16-bit 250-MS/s IF sampling pipelined ADC With background calibration," in Solid-State Circuits, IEEE Journal of, vol. 45, no. 12 , pp. 2602-2612, Dec. 2010. 
[13] J. Kauffman, P. Witte, J. Becker, and M. Ortmanns, "An 8mW 50MS/s $\mathrm{CT} \Delta \Sigma$ modulator with $81 \mathrm{~dB}$ SFDR and digital background DAC linearization," in Solid-State Circuits Conference Digest of Technical Papers (ISSCC), 2011 IEEE International, Feb. 2011, pp. 472-474.

[14] M. Park and M. Perrott, "A 78 dB SNDR $87 \mathrm{~mW} 20 \mathrm{MHz}$ bandwidth continuous-time $\Delta \Sigma \mathrm{ADC}$ with VCO-based integrator and quantizer implemented in $0.13 \mu \mathrm{m}$ CMOS," in Solid-State Circuits, IEEE Journal of, vol. 44, no. 12, pp. 3344-3358, Dec. 2009.

[15] P. Malla, H. Lakdawala, K. Kornegay, and K. Soumyanath, "A 28mW spectrum-sensing reconfigurable 20MHz 72dB-SNR 70dB-SNDR DT $\Delta \Sigma$ ADC for 802.11n/WiMAX receivers," in Solid-State Circuits Conference, 2008. ISSCC 2008. Digest of Technical Papers. IEEE International, Feb. 2008, pp. 496-631.

[16] G. Mitteregger et al., "A 20-mW 640-MHz CMOS continuous-time ADC With 20-MHz signal bandwidth, 80-dB dynamic range and 12-bit ENOB," in Solid-State Circuits, IEEE Journal of, vol. 41, no. 12, pp. 2641-2649, Dec. 2006.

[17] A. Hart and S. Voinigescu, "A $1 \mathrm{GHz}$ bandwidth low-pass $\Delta \Sigma$ ADC with 20-50 GHz adjustable sampling rate," in Solid-State Circuits, IEEE Journal of, vol. 44, no. 5, pp. 1401-1414, May 2009.

[18] S. Krishnan et al., "An 8-GHz continuous-time $\Sigma \Delta$ analog-digital converter in an InP-based HBT technology," in Microwave Theory and Techniques, IEEE Transactions on, vol. 51, no. 12, pp. 2555-2561, Dec. 2003.

[19] J. Harrison et al., "An LC bandpass $\Delta \Sigma$ ADC with 70dB SNDR over 20MHz bandwidth using CMOS DACs," in Solid-State Circuits Conference, 2012. ISSCC 2012. Digest of Technical Papers. IEEE International, Feb. 2012, pp. 146-147.

[20] J. Chae, H. Jeong, G. Manganaro, and M. Flynn, "A 12mW low-power continuous-time bandpass $\Delta \Sigma$ with $58 \mathrm{~dB}$ SNDR and $24 \mathrm{MHz}$ bandwidth at 200MHz IF," in Solid-State Circuits Conference, 2012. ISSCC 2012. Digest of Technical Papers. IEEE International, Feb. 2012, pp. 148-149.

[21] H. Shibata et al., "A DC-to- $1 \mathrm{GHz}$ tunable RF $\Delta \Sigma$ ADC achieving $\mathrm{DR}=74 \mathrm{~dB}$ and $\mathrm{BW}=150 \mathrm{MHz}$ at $f_{0}=450 \mathrm{MHz}$ using $550 \mathrm{~mW}$," in SolidState Circuits Conference, 2012. ISSCC 2012. Digest of Technical Papers. IEEE International, Feb. 2012, pp. 150-151. 
[22] K. Reddy et al., "A 16mW 78dB-SNDR 10MHz-BW CT- $\Delta \Sigma$ ADC using residue-canceling VCO-based quantizer," in Solid-State Circuits Conference, 2012. ISSCC 2012. Digest of Technical Papers. IEEE International, Feb. 2012, pp. 152-153.

[23] P. Witte et al., "A 72dB-DR $\Delta \Sigma \mathrm{CT}$ modulator using digitally estimated auxiliary DAC linearization achieving $88 \mathrm{fJ} / \mathrm{conv}$ in a $25 \mathrm{MHz} \mathrm{BW}$," in Solid-State Circuits Conference, 2012. ISSCC 2012. Digest of Technical Papers. IEEE International, Feb. 2012, pp. 154-155.

[24] P. Shettigar and S. Pavan, "A $15 \mathrm{~mW} 3.6 \mathrm{GS} / \mathrm{s}$ CT- $\Delta \Sigma$ ADC with $36 \mathrm{MHz}$ bandwidth and 83 DR in 90nm CMOS," in Solid-State Circuits Conference, 2012. ISSCC 2012. Digest of Technical Papers. IEEE International, Feb. 2012, pp. 156-157.

[25] V. Srinivasan et al., "A 20mW 61dB SNDR (60MHz BW) 1b $3^{\text {rd } \text {-order }}$ continuous-time bandpass delta-sigma modulator clocked at $6 \mathrm{GHz}$ in $45 \mathrm{~nm}$ CMOS," in Solid-State Circuits Conference, 2012. ISSCC 2012. Digest of Technical Papers. IEEE International, Feb. 2012, pp. 158-159. 


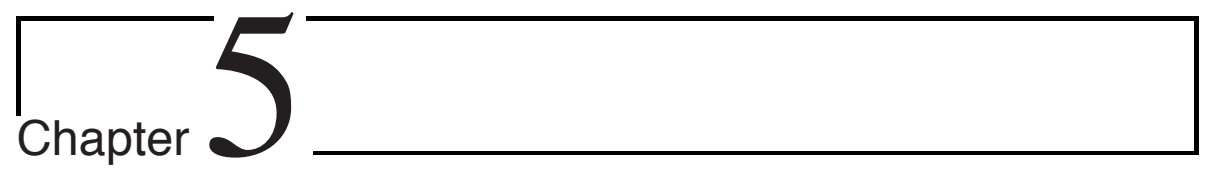

\section{A $2 \mathrm{GHz}$ Continuous-Time $\Delta \Sigma$ ADC with Dynamic Error Correction}

In the previous chapter, we have presented the design and implementation details of a $3^{\text {rd }}$ order 4-bit continuous-time delta-sigma ( $\mathrm{CT} \Delta \Sigma$ ) ADC which uses a high-speed filter topology. However, its signal-to-noise ratio (SNR) and signal-to-noise-and-distortion ratio (SNDR) are limited to 65.5 and $65 \mathrm{~dB}$ at $-0.5 \mathrm{dBFS}$ input, respectively. The main reason for this is that for large input signals, the non-linearity of the 4-bit feedback DAC (DAC1) causes harmonic components and quantization noise to fold into the signal band, which increases the in-band noise. In order to improve the SNR and SNDR of the modulator, the non-linearity of the multi-bit DAC1 must be tackled. This chapter discusses how to do this, and in particular, how to improve the high frequency linearity of DAC1 without degrading the stability of the modulator.

This chapter starts with an overview of error sources in DAC1. For each error source, different types of calibration/correction techniques can be applied. In Section 5.2, we focus on dynamic error correction techniques and discuss the trade-offs between different techniques. The stringent stability requirement of a high-speed delta-sigma modulator $(\Delta \Sigma \mathrm{M})$ limits implementable error correction techniques. Furthermore, we describe the proposed dynamic error correction technique which is the focus of the remaining part of this chapter. Section 5.3 describes the modifications required to sample the modulator at $2 \mathrm{GHz}$, which is half of the original sampling rate, while simultaneously correcting the 


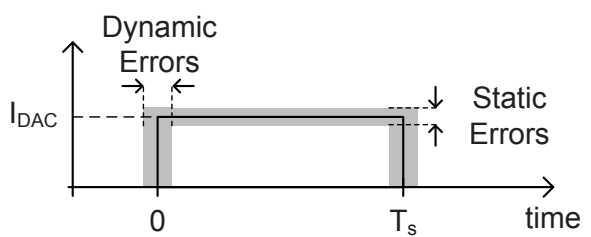

Figure 5.1 - Conceptual model of dynamic and static errors in a non-returnto-zero DAC pulse.

dynamic errors of DAC1. Section 5.4 then describes the implementation details of the modulator architecture and the proposed dynamic error correction technique. The experimental results are described in Section 5.5.

\section{$5.1 \quad$ Introduction}

DAC1 is the most dominant source of non-linearity of the modulator, as explained in Chapter 3.2.3. Designing low-noise, linear high-speed DACs has received considerable attention over the years [1-4]. However, most of the design techniques have been developed for applications which utilize such DACs in an open-loop configuration, where their latency is not a critical design consideration.

Figure 5.1 shows the output current of a non-return-to-zero (NRZ) DAC, where the static (amplitude) and dynamic (timing) errors are illustrated. Both static and dynamic errors effect the performance of the modulator and correcting only one of these is not sufficient to achieve good performance at high frequencies. One can argue that static errors are more dominant at low frequencies. However, it is theoretically possible that static and dynamic errors may cancel each other for a certain input frequency and sampling frequency combination. On the other hand, dynamic errors become more dominant as the sampling frequency of the modulator increases, especially beyond GHz. Therefore, to achieve the full performance of the modulator, both types of errors must be suppressed or corrected.

\section{Static Errors and Overview of Correction Techniques}

In this work, we focus on a current-steering DAC, since it is the preferred architecture at high sampling rates $[1,5]$. Fig. 5.2 shows the schematic of an N-bit thermometer coded current-steering DAC. The mismatch in the output 


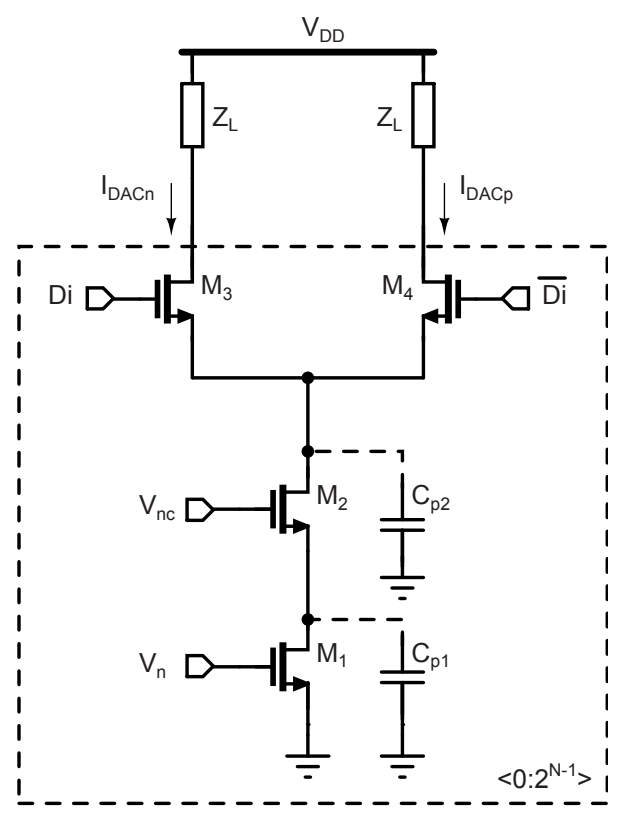

Figure 5.2 - Schematic of a current-steering thermometer-coded DAC.

currents of the DAC elements is the most dominant type of static error. The output of each current source deviates from its originally designed value due to process variation, lithography errors, and layout artifacts. Increasing the transistor sizes reduces current mismatch. However, beyond 10 bits of matching, the area required for the current source transistor $\left(M_{1}\right)$ becomes so large that global variations and temperature gradients become dominant compared to local device mismatch errors $[5,6]$.

In addition to current mismatch, the data-dependent output impedance of the DAC introduces static errors [1]. Assume that the input data of the DAC is between $0-N$, where $\mathrm{N}$ is the total number of thermometer-coded DAC units, which is equal to 15 in the case of a 4-bit unary weighted DAC. All the current sources are connected to the positive output of the DAC when the input data is $N$ and following the same reasoning, if the input data is 0 , then all the current sources are connected to the negative output of the DAC. As the input data of the DAC changes, the number of current sources connected to the output is modulated, which also modulates the output impedance of the DAC. In order to increase the high-frequency output impedance of the current sources, which are scaled for matching, a cascode transistor $\left(M_{2}\right)$ is employed. 
However, at $\mathrm{GHz}$ sampling frequencies, the output impedance of the DAC will be dominated by the switch capacitance. The $3^{r d}$ order harmonic distortion $\left(\mathrm{HD}_{3}\right)$ due to the limited output impedance is expressed as [3,7]:

$$
H D_{3}=\left(\frac{Z_{L} N}{4\left|Z_{\text {out }}\right|}\right)^{2},
$$

where $Z_{L}$ is the load impedance, $\mathrm{N}$ is the number of current sources, and $Z_{\text {out }}$ is the output impedance of the unit current source. In a stand-alone DAC, $Z_{L}$ is set externally and a $50 \Omega$ termination impedance is often used. As a result, the output impedance of the DAC defines the maximum $\mathrm{HD}_{3}$ specification for a given number of current sources. In the case of a $\Delta \Sigma \mathrm{M}$, the DAC is often connected to the virtual ground node of an active-RC integrator whose input impedance acts as the load impedance $\left(Z_{L}\right)$ of the DAC. The input impedance of the integrator is approximately equal to $1 / g_{m}$, which can be designed to be much lower than $50 \Omega$ if necessary.

The static errors in a multi-bit DAC introduce harmonic tones and degrade the linearity of the modulator. As described in Section 2.2.2, the mismatch of a multi-bit DAC especially limits the low-frequency linearity of the modulator. Theoretically, the resulting total harmonic distortion (THD) and spurious-free dynamic range (SFDR) of the modulator do not scale with input frequency $\left(f_{i n}\right)$ or sampling frequency $\left(f_{s}\right)[2]$. However, as the frequency of the input signal increases, the tones generated by the static errors will move outside of the signal bandwidth (BW) and will be filtered by the decimation filter. The output impedance of the DAC decreases as the input frequency of the signal increases, which degrades the THD and SFDR of the modulator. However, device capacitances reduce for every new generation of nm-CMOS technology, hence the $f_{T}$ of the technology improves. Therefore, the high-frequency output impedance of a multi-bit DAC will improve with process $[3,8]$.

In order to reduce the effect of static errors, various design techniques can be applied as shown in Fig. 5.3. First of all, the mismatch errors of the DAC can be minimized by scaling the transistors, and dividing each current source into smaller blocks, which can then be placed in a random pattern that will minimize global mismatch and gradient errors. However, the complexity and parasitic capacitance of the layout increases which might also increase the latency of the DAC. On the other hand, achieving beyond 10-11 bits of matching thus requires additional calibration or correction of current-source mismatch. Since the input data of a DAC is known in advance, the switching sequence of the DAC units can be optimized to improve the linearity. Techniques such as dynamic element matching (DEM), data weighted averaging (DWA) [9] and mapping alter the switching sequence of the DAC units in order to linearize its 


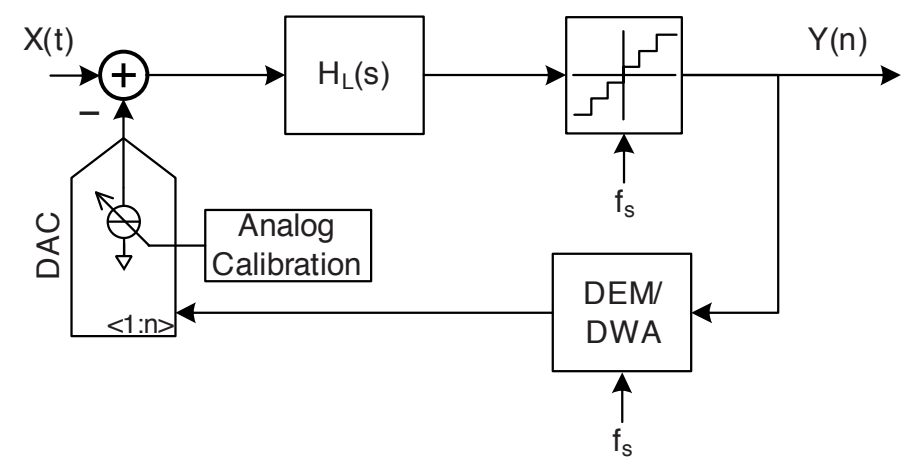

Figure 5.3 - DAC calibration/correction techniques in a single-loop $\mathrm{CT} \Delta \Sigma$ modulator.

transfer characteristic $[10,11]$, improve the low-frequency THD and SFDR of the modulator. However, these techniques require decoders in the data path of the DAC, and propogation delay of these decoders increases the total latency in the feedback path. Therefore, modulators sampled beyond $1 \mathrm{GHz}$ do not rely on digital correction techniques to achieve high linearity because they degrade the stability of the modulator.

Current-source mismatch can be reduced by using analog calibration techniques. The current mismatch of each DAC unit is measured and compensated for by an auxiliary current source whose gate voltage is controlled by the measured current mismatch [12]. Analog calibration techniques often increase the layout area of the DAC and require a certain refresh rate to maintain a constant calibration current. On the other hand, a high dynamic range (DR) modulator can also measure the mismatch of its own DAC units and correct the mismatch errors by using an auxiliary DAC [13]. However, the dynamic (timing) mismatch between the main and auxiliary DAC limits the high-frequency linearity of the modulator.

\section{Dynamic Errors and Overview of Correction Techniques}

The dynamic errors of a DAC are defined by the switching behavior of its current sources. Many factors are responsible for this: current-source mismatch, switch mismatch, a non-ideal layout, clock skew, supply and clock-tree imbalance. Most of these errors can be minimized by addressing them at the design level. However, the latency of the DAC must not be degraded because of the stability requirements of the $\Delta \Sigma \mathrm{M}$. 
Systematic design approaches can be employed to reduce the effect of dynamic errors in a multi-bit DAC, but the random device mismatch affects the exact switching moment of the DAC current sources. Dynamic errors introduce harmonic tones and degrade the linearity of the modulator. When compared to static errors, dynamic errors become more and more dominant as the sampling frequency increases [2], therefore the SNR, SNDR, and SFDR will drop at high frequencies. However, if the harmonic tones generated by the dynamic errors fall outside of the signal BW, the decimation filter filters them out and the SFDR of the $\Delta \Sigma \mathrm{M}$ will improve unlike the situation in Nyquist converters, where the tones that move out of the first Nyquist zone will fold into the signal BW. On the other hand, due to the non-linearity of the modulator, some of the quantization noise will be down-converted to the baseband and degrade the SNR and SNDR of the modulator.

Calibrating or correcting all the timing errors is a very challenging objective. Figure 5.1 shows the dynamic errors which are modeled as uncertainty in the switching moment of DAC output. In order to reduce the effect of dynamic errors, the rise/fall time of the DAC output must be minimized as much as possible [14]. The switching speed of a DAC implemented in nm-CMOS technology definitely benefits from the high $f_{T}$ of the process. To generate steep rising/falling edges, the DAC driver with several cascaded master-slave latches is preferred. However, additional latching stages increase the latency of the $\mathrm{DAC}$, which is not acceptable due to the stability requirements of the $\Delta \Sigma \mathrm{M}$ as explained in Chapter 2

If the dynamic errors of the DAC units are known, these errors can be calibrated or corrected. For example, the dynamic error of a DAC unit can be measured by comparing its output to the output of a reference DAC unit. If a dynamic error exists, the result of the comparison will be non-zero. By using this information, an improved switching sequence of DAC units can be applied which will result in a better high-frequency linearity [2]. However, the decoder required to change the switching sequence introduces latency, and the limited number of DAC units ( $\Delta \Sigma \mathrm{Ms}$ often use DACs with less than 5-bit resolution) reduces the yield of the correction technique.

Furthermore, the output of the DAC and high-speed clock couple to the biasing lines of the DAC which creates signal-dependent distortion. Especially the glitches at the common source of the data switches modulate the DAC output current. Due to the mismatch of these switches, each DAC unit effectively has a different crossing point which affects the switching moment of the DAC output. This crossing point of each data switch can be optimized by using a measurement and control circuitry which aim to reduce the glitch present at the common source of the switches [4]. However, the measurement circuitry 
must be connected to the common source of the data switches, which degrades the high-frequency output impedance of the DAC.

In addition to the above-mentioned techniques which measure dynamic errors and correct them, the effect of dynamic errors can be mitigated by isolating them from the DAC's output. A return-to-zero (RZ) switching scheme, which disconnects the DAC output for a fraction of the sampling period, can be used to improve the linearity of the DAC. However, since the DAC is used as the feedback of the $\Delta \Sigma \mathrm{M}$, the system-level and circuit level trade-offs must analyzed. In the next section, we will focus on dynamic error correction techniques which rely on the RZ switching scheme and discuss its feasibility at high sampling rates.

\subsection{Dynamic Error Correction Techniques in $\Delta \Sigma$ Modulators}

As explained in the previous section, most of the dynamic-error correction techniques have been developed for stand-alone DACs whose the latency is not a critical design parameter $[3,7,8,14,15]$. High-speed, wide-BW $\Delta \Sigma \mathrm{Ms}$ often use a multi-bit differential current steering DAC architecture $[16,17]$ which reduces the inter-symbol interference (ISI) $[18,19]$. In a differential current steering DAC which toggles every clock period, non-equal rise and fall times do not effect the charge present at the DAC output. However, if data do not toggle, then the charge delivered to the output of the DAC is data-dependent, which results in a signal-dependent non-linearity. To solve the data-dependent nonlinearity, the DAC output can be switched two times for every clock period (differential quad switching (DQS) as shown in Fig. 5.4), so that the error introduced does not depend on data but the error energy is located at double the clock frequency $[4,20]$. DQS improves the dynamic linearity of single-bit DACs. In the case of a multi-bit DAC, the mismatch in the rise/fall time of different DAC units sets a limit on the maximum achievable linearity.

The RZ switching scheme is a modified version of the DQS scheme where the output of the DAC is reset during every clock period such that the DAC output does not depend on the previous data activity. Figure 5.5 shows a possible implementation of a RZ DAC connected to the input stage of a CT $\Delta \Sigma$ modulator. The first stage of the modulator is implemented as an active-RC integrator. During every clock period, each DAC unit is disconnected from the virtual ground node of the amplifier and the output current is dumped into a common mode voltage which has ideally the same potential as the input common mode $(\mathrm{CM})$ of the amplifier. 


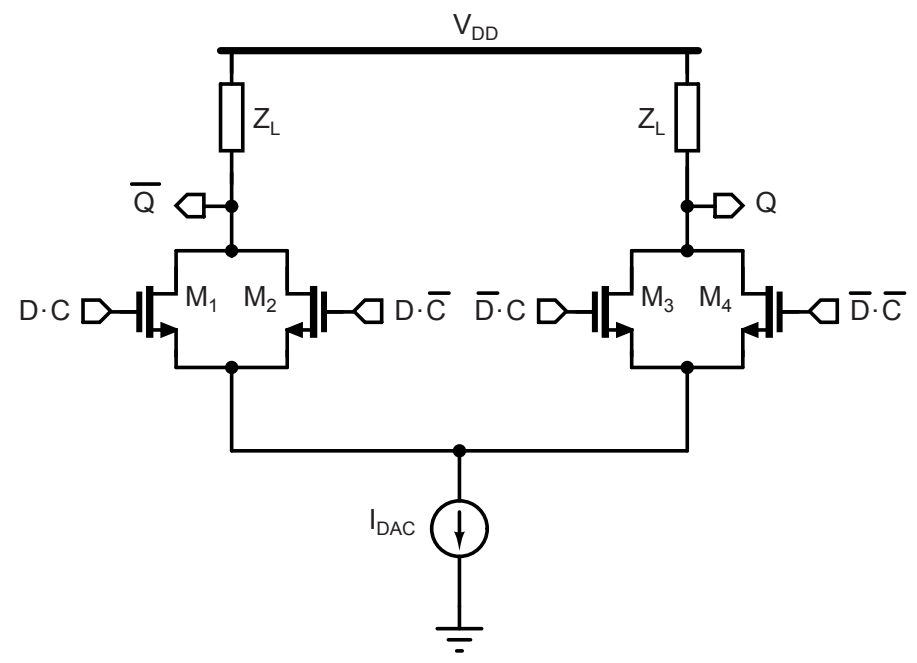

(a)

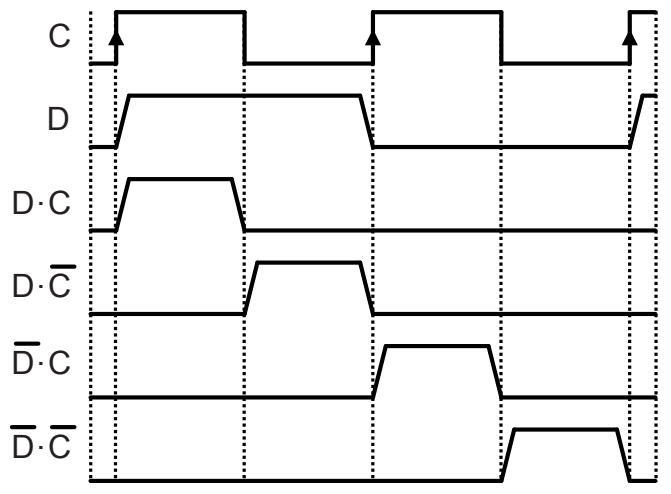

(b)

Figure 5.4 - Simplified DAC unit based on differential-quad switching (DQS) (a). Timing diagram of the DQS scheme (b). 


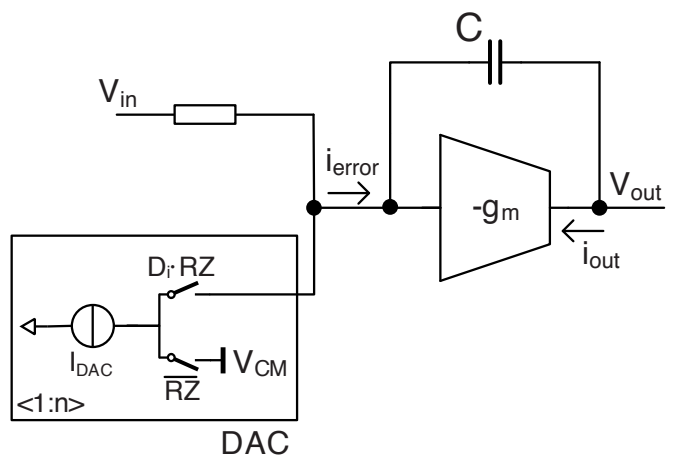

Figure 5.5 - The input stage of a $\Delta \Sigma \mathrm{M}$ with a return-to-zero (RZ) DAC. The schematic is drawn as a single-ended configuration but in the real implementation a fully differential configuration is preferred.

Before analyzing a $\Delta \Sigma \mathrm{M}$ with the RZ DAC, we will summarize the various advantages of a multi-bit NRZ DAC. Fig. 5.6 shows the transient simulation result of the $3^{r d}$ order feedforward $\Delta \Sigma$ modulator with the multi-bit NRZ DAC described in Chapter 3. The feedback DAC current $\left(I_{D A C}\right)$ follows the input current $\left(I_{i n}\right)$, and the maximum value of the error current $\left(I_{\text {error }}\right)$ does not exceed $1-2 I_{L S B}$. The $\Delta \Sigma \mathrm{M}$ with the multi-bit NRZ DAC is less sensitive to clock jitter since the DAC output toggles only 1-2LSB per transition, which reduces the error signal injected into the input of the modulator for every clock pulse. Moreover, the error signal that the loop filter integrates is inversely related to the resolution of the DAC. As the error signal reduces, the BW and slew rate requirements of the first amplifier reduce along with the power dissipation.

Figure 5.7 shows the simulation results of the RZ DAC illustrated in Fig. 5.5. The $I_{D A C}$ still follows $I_{i n}$, but when the RZ pulse is zero, the error current is effectively equal to $I_{i n}$. The RZ switching scheme eliminates ISI-related nonlinearity, although the error current that is integrated by the input stage of the loop filter has much more high-frequency content. This increases the slew rate and $\mathrm{BW}$ requirements of the first amplifier. Moreover, when the DAC is connected to the CM, basically the $\Delta \Sigma$ loop is disconnected and the outputs of the loop filter exhibit larger output swings which can cause headroom problems and require the scaling of the loop-filter coefficients. For modulators with low oversampling ratio (OSR), the increase in the output swings of the loop filter stages becomes larger since the loop filter provides more gain compared to 

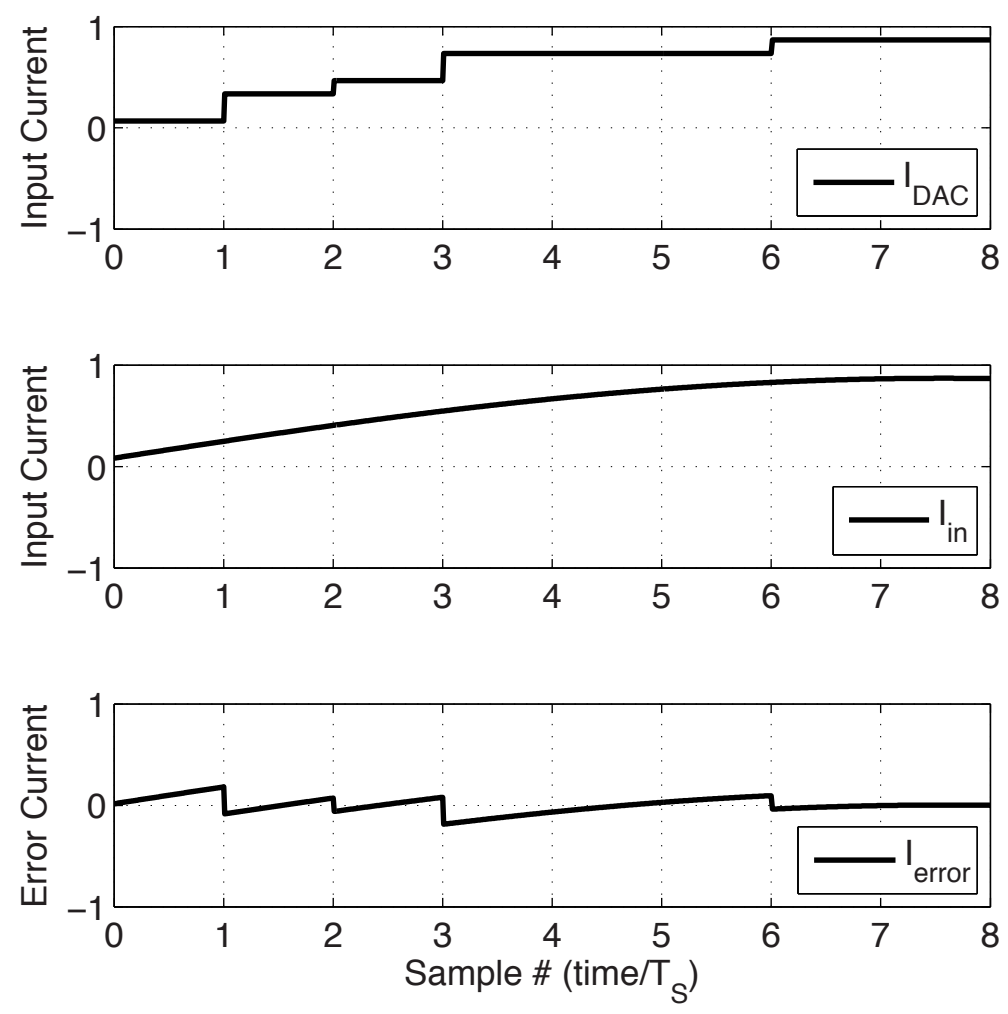

Figure 5.6 - The input current $\left(I_{i n}\right)$ and DAC output current $\left(I_{D A C}\right)$ of a $3^{\text {rd }}$ order single-loop CT $\Delta \Sigma$ modulator with a 4-bit quantizer with the non-returnto-zero (NRZ) switching scheme and the input error current $\left(I_{\text {error }}=I_{\text {in }}-I_{D A C}\right)$ of the first integrator of the modulator. 

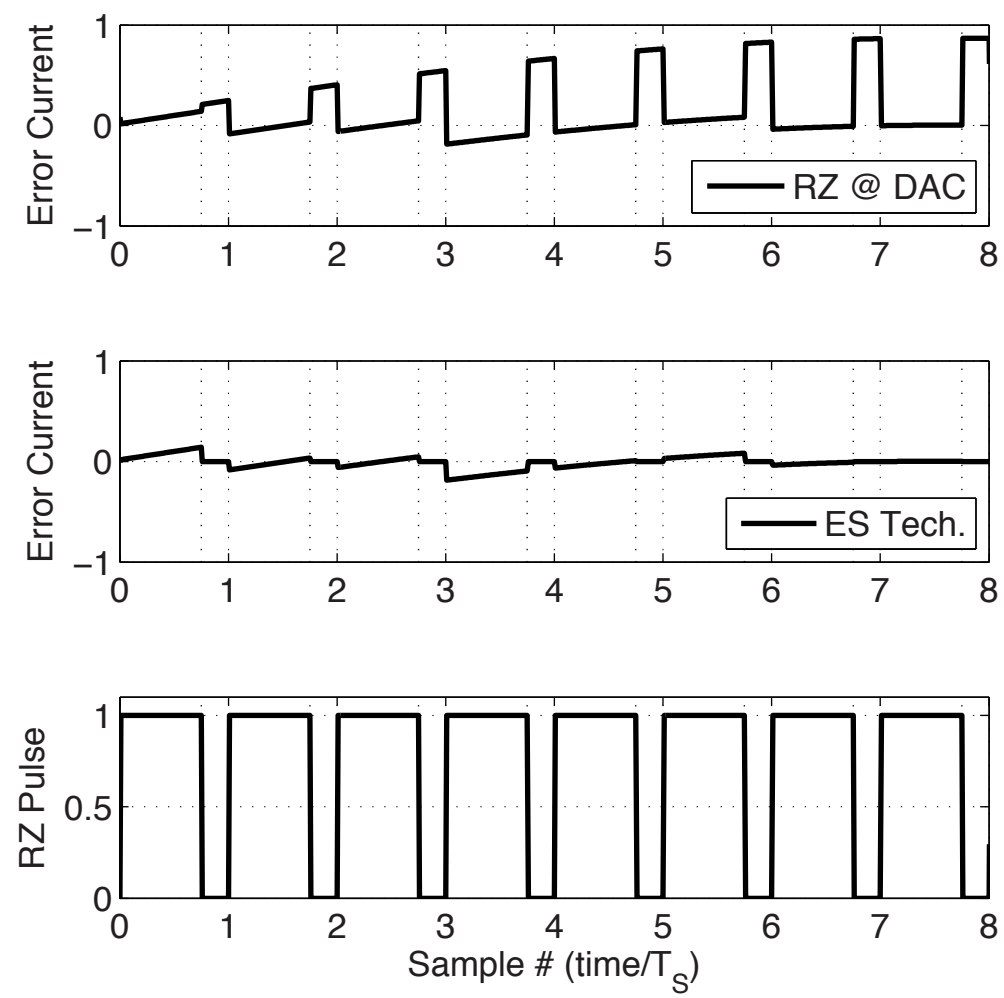

Figure 5.7 - The input current of the first integrator of a $3^{\text {rd }}$ order single-loop $\mathrm{CT} \Delta \Sigma$ modulator with a 4-bit quantizer with the return-to-zero (RZ) switching scheme. 


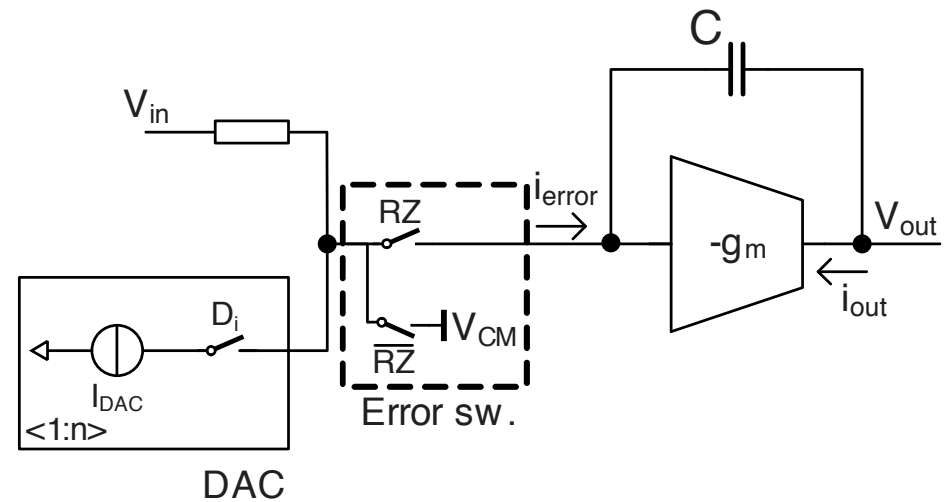

Figure 5.8 - The input stage of a $\Delta \Sigma \mathrm{M}$ with an error switch clocked with a return-to-zero (RZ) clock pulse.

modulators with high OSR. All these drawbacks degrade the performance and increase the power consumption of a high-speed $\Delta \Sigma \mathrm{M}$.

However, the RZ switching scheme only relaxes the ISI requirement of the DAC. The other dynamic errors related to timing, clock and supply tree unbalance, and mismatch of the data switches will still limit modulator performance at very high sampling rates. In order to reduce the contribution of these dynamic errors, the RZ switching scheme can be modified such that a master RZ switch can replace the distributed RZ switches at the output of each DAC unit. However, the DAC output still disconnects from the integrator when the RZ pulse is 0 , which increases the requirements of the first integrator.

\subsubsection{The Error Switching Technique}

The RZ switching scheme corrects the ISI errors of a multi-bit DAC, which is one of the dominant sources of dynamic errors at high sampling rates. However, applying a RZ switching scheme increases the power consumption of the modulator since the input current of the first integrator increases. Furthermore, applying RZ switching scheme only to the DAC output increases the clock jitter-induced errors and reduces the DR performance of the modulator. In order to solve these drawbacks, the RZ switching scheme is applied to the 
error signal $^{1}$ of the first integrator, as shown in Fig. 5.8, where the error signal is the difference between the input signal and the DAC signal. This approach has been used in a hybrid CT $\Delta \Sigma$ modulator in order to solve ISI and compensate for the sample rate variation caused by process, voltage, and temperature (PVT) variations with a maximum $f_{s}$ of $6.144 \mathrm{MHz}$ which targets audio applications [21]. Such a sampling frequency is much smaller than the cutoff frequency $\left(f_{T}\right)$ of the process technology. Applying the error switching (ES) technique at gigahertz sampling frequency will bring its own challenges. Moreover, it has not been practically demonstrated that the ES technique improves the dynamic performance of a high-speed $\Delta \Sigma \mathrm{M}$.

Figure 5.7 shows simulation results of the RZ DAC and the ES scheme depicted in Figs. 5.5 and 5.8, respectively. Both the ES and RZ scheme use the same RZ pulse to simplify the comparison. The switches are ON when the $\mathrm{RZ}$ pulse is 1 . The error current of the ES technique does not exhibit large peaks when compared to that of the RZ switching scheme. Therefore, the slew rate and BW requirements on the first integrator's amplifier do not increase. Furthermore, similar scaling coefficients can be used for the loop filter, since the first integrator's output does not generate large uncontrolled signal levels.

Basically, the working principle of the first integrator does not change, but it integrates the error current when the rising edge of the RZ pulse arrives. The DAC and the input signal are disconnected at the falling edge of the RZ pulse and connected to the CM voltage, which can be generated by on-chip reference circuitry. If the DAC resamples the new data when it is connected to the CM voltage, assuming that all the dynamic errors settle when the RZ pulse is 0 , then the first integrator basically does not process the dynamic errors. Therefore, the ES technique can eliminate all the dynamic errors present in a high-speed $\Delta \Sigma \mathrm{M}$ if all the dynamic errors settle when the error current of the integrator is connected to the CM voltage.

Figure 5.9a shows an FFT of the simulated output of a 4-bit $3^{r d}$ order $\Delta \Sigma$ analog-to-digital converter (ADC) with a timing mismatch of $\sigma_{T_{D A C}} / T_{D A C}=$ $0.5 \%$. The modulator does not have any static mismatch error. It can be seen that the timing mismatch of the DAC limits the linearity of high-speed $\Delta \Sigma \mathrm{M}$. By using the ES technique, these non-linearity errors can be removed. Fig. $5.9 \mathrm{~b}$ shows the simulated output of the modulator. All non-idealities due to the timing mismatch are removed.

On the other hand, the integrator processes the signals which are switched, therefore the signals around $f_{s} \pm f_{s} / O S R$ aliases down to the signal BW, which is the major drawback of this technique. However, the $\Delta \Sigma \mathrm{M}$ is an oversampled

\footnotetext{
${ }^{1}$ In this work, we prefer to call it an error-switching (ES) technique because it more suitably describes the working principle of the scheme.
} 


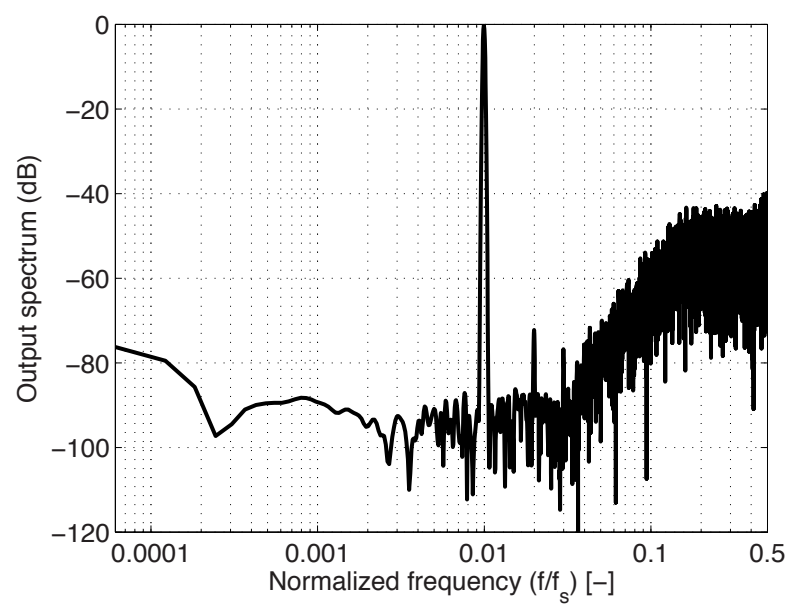

(a)

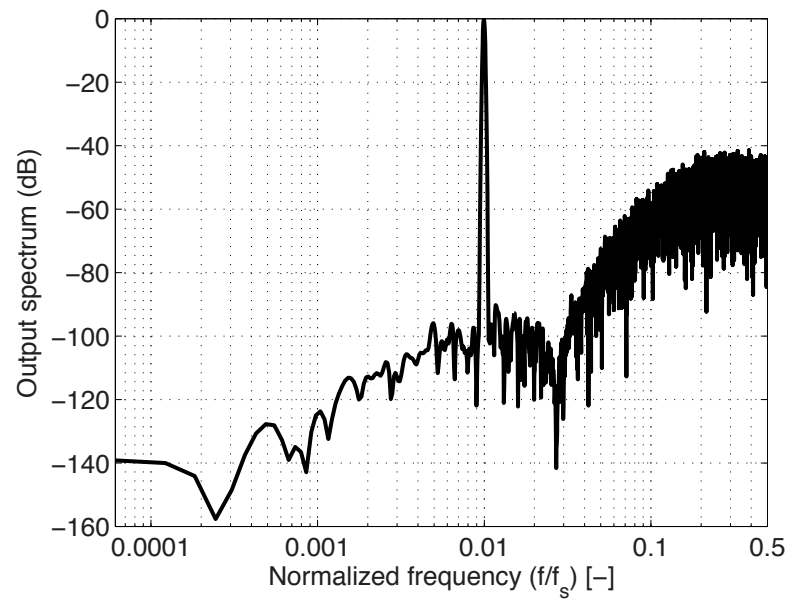

(b)

Figure 5.9 - The harmonic tones due to the dynamic mismatch of a multi-bit DAC.(a). An FFT of the simulated $\Delta \Sigma \mathrm{M}$ with ES technique applied. (b). (FFT size is $2^{17}$ pts.) 
system, and the aliasing filter requirement is still relaxed compared to Nyquist converters. The next section describes the design of a high-speed $\Delta \Sigma \mathrm{M}$ which is sampled at $2 \mathrm{GHz}$, along with architectural modifications required for the ES technique, and the associated circuits.

\subsection{Multi-Mode High-Speed $\Delta \Sigma$ ADC Design}

This section describes the system-level modifications required to run a $\mathrm{CT} \Delta \Sigma$ modulator with an ES scheme. Since, we would like to investigate the ES technique described in previous section, which requires an RZ signal with very short pulse widths $\left(10-20 \% T_{S}\right)$, we have decided to implement a multi-mode high-speed $\mathrm{CT} \Delta \Sigma$ modulator which can be sampled with a clock frequency between $2-4 \mathrm{GHz}$. When the ES technique is turned ON, the ADC is clocked at $2 \mathrm{GHz}$. At a lower sampling frequency, the dynamic non-idealities of the circuits apart are much less which allows us to focus on the effectiveness of the ES technique. In Chapter 3 and 4, we have described the design of a high-speed modulator clocked at $4 \mathrm{GHz}$. Therefore, in this section, we will focus on the modification required to clock such a modulator at half of its original sampling rate.

Figure 5.10 shows the system-level model of the $\mathrm{CT} \Delta \Sigma$ modulator with an error switch and high-speed pulse generator. The details of the error switch block and pulse generator are explained in Section 5.4. In order to implement a stable CT modulator clocked at half of the sampling rate, the poles of the loop filter must be scaled down by $2 x$. To achieve this, the capacitors of the first and second integrators must be scaled up by $2 x$ while keeping the same integrator resistors such that the modulator has the same input noise density. Furthermore, the last integrator's pole is implemented with fixed capacitors $\left(C_{T}=C_{A 0}+C_{A 1}+C_{A 2}\right)$ that are connected to the output of the loop filter. Therefore, the $g_{m}$ of the last integrator must be scaled down by $2 x$.

The main feedback DAC (DAC1) is not modified since the input impedance of the first integrator is kept the same. The excess loop delay (ELD) compensation DAC (DAC2) output current must be scaled down by $2 x$, since the output impedance of the loop filter is defined by the feedforward capacitors $\left(C_{T}=C_{A 0}+C_{A 1}+C_{A 2}\right)$, and the effective output impedance increases by $2 x$ when clocked at half of the original sampling rate. Instead of reducing the output current of DAC2, the differentiation duration of DAC2 can also be reduced by $2 x$ to achieve the same feedback coefficient. However, adjusting the differentiation duration is not advantageous since it requires additional circuitry, increases the complexity of the design, and reduces the power efficiency of the 


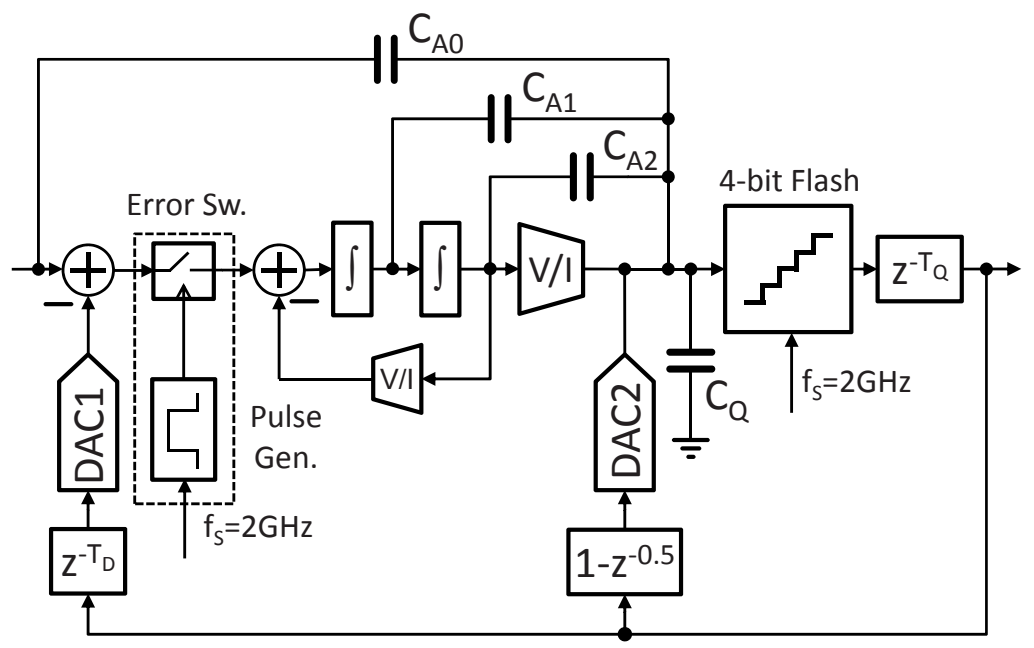

Figure 5.10 - The proposed high-speed capacitive feedforward CT $\Delta \Sigma$ modulator with an error switch at the input of the loop filter.

modulator.

The circuit blocks such as the 4-bit quantizer, decoder, and decimation filter are not affected by the sampling rate modification. However, the modulator requires a new timing diagram such that it can achieve the best performance without degrading the stability. Figure 5.11 shows the timing ${ }^{2}$ diagram of the modulator at $2 \mathrm{GHz}$ with the ES mode activated. At the rising edge of the $R S T$ signal, the quantizer starts sampling, and half a clock period later the data is available at its output. DAC1 samples the output of the quantizer at the rising edge of the $C L K_{D A C 1}$, which must arrive before the rising edge of the $R S T$ signal. The rising edge of the $C L K_{D A C 1}$ and $R Z$ is aligned such that all of the DAC current sources switch their outputs $\left(D_{<i>}\right)$ as shown in Fig. 5.11. The OFF duration of the $R Z$ pulse $\left(\Delta T_{R Z}\right)$ is defined by the settling speed of the dynamic errors present at the output of the DAC, which is extracted by circuit-level simulations. Furthermore, the overall timing of the modulator is set by adjusting the delay of the tunable clock buffers.

\footnotetext{
${ }^{2}$ The timing of DAC2 and quantizer, which have not been modified, are shown in Figs. $3.27 \mathrm{~b}$ and 4.4 , respectively.
} 


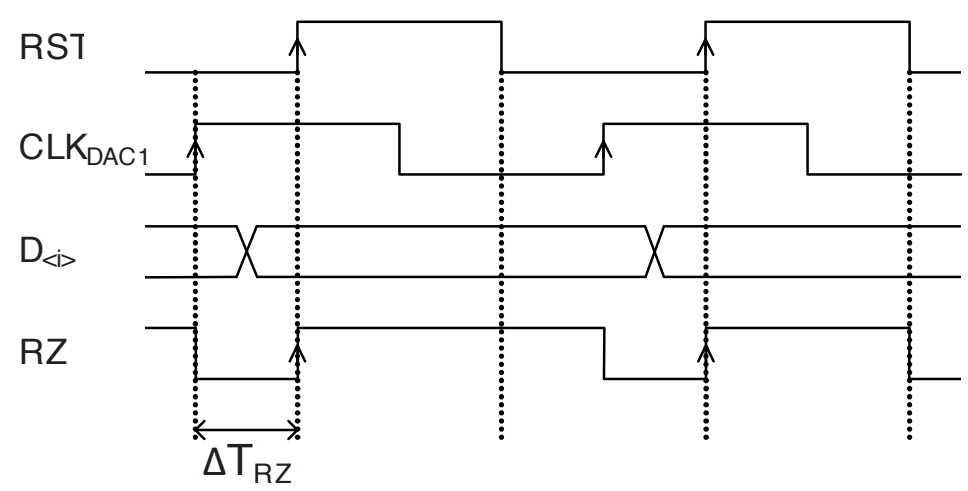

Figure 5.11 - The timing diagram of the CT $\Delta \Sigma$ modulator with error sampling switch.

\subsection{Implementation Details}

Figure 5.12 shows the top level architecture of the $3^{r d}$ order $\mathrm{CT} \Delta \Sigma$ ADC with the error switch at the input of the loop filter and the high-speed pulse generator. The ADC is clocked at $2 \mathrm{GHz}$ when the ES mode is enabled. In order to achieve multi-mode operation of the ADC, the modifications presented in Section 5.3 have been implemented at the architectural level. To enable multi-mode operation and compensate for RC spread, $C_{1}, C_{2}$ and $R_{3}$ can be individually tuned via 5 -bit networks, for which the implemented tuning range covers $0.5-2 \times$ of the nominal RC time constant. The third integrator is a $g_{m} C$ integrator whose load capacitance is fixed. To achieve a stable modulator at half of the original sampling rate, the $g_{m}$ of the last integrator is tuned by adjusting its bias current. The implemented tuning range is $0.5-2 g_{m}$.

DAC2 is directly connected to the capacitive summing node. Since the impedance at its output scales inversely proportionally to the sampling frequency $\left(Z_{\text {out }} \propto 1 / \omega_{s} C_{\text {TOTAL }}\right)$, its output current must be scaled proportionally to the sampling frequency. The output current of DAC2 is scaled by tuning its bias current externally.

\subsubsection{Input Stage and the Loop Filter}

Figure 5.13 shows the schematic of the input stage of the $3^{\text {rd }}$ order CT $\Delta \Sigma$ modulator with its feedback DAC and error switches. The error switches are scaled such that their thermal noise contribution is negligible. The modulator 


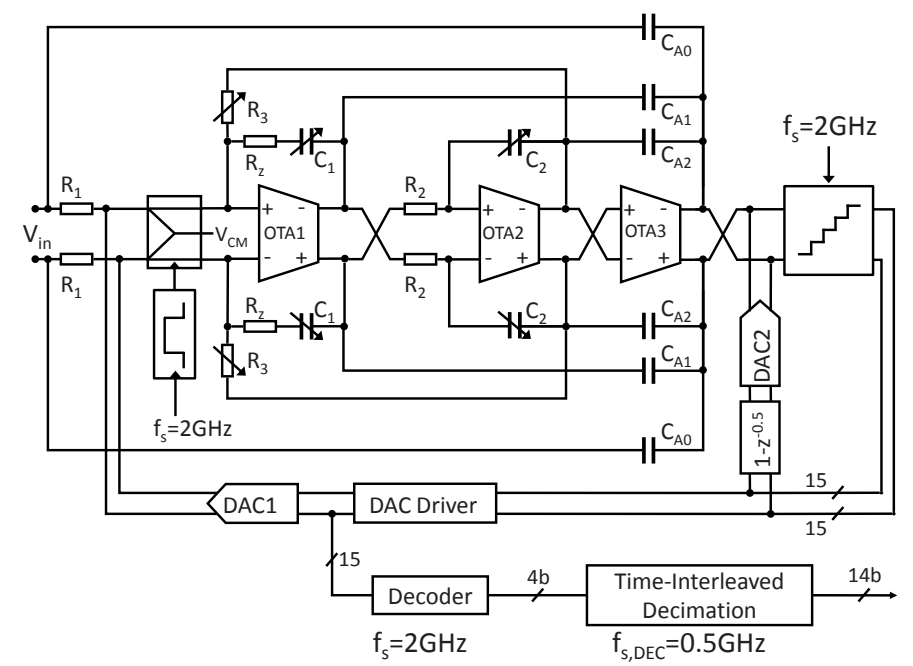

Figure 5.12 - The top-level architecture of the $3^{r d}$ order $\mathrm{CT} \Delta \Sigma$ ADC with an error switch at the input of the loop filter.

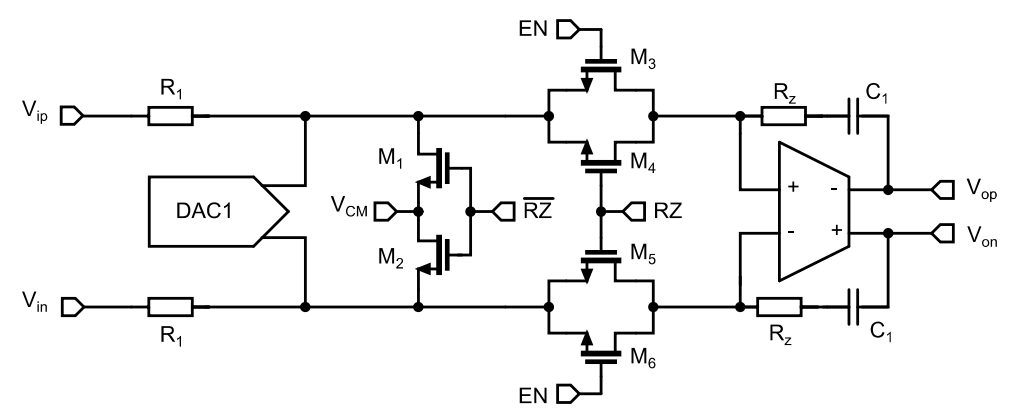

Figure 5.13 - Input stage of the modulator with error switch. 


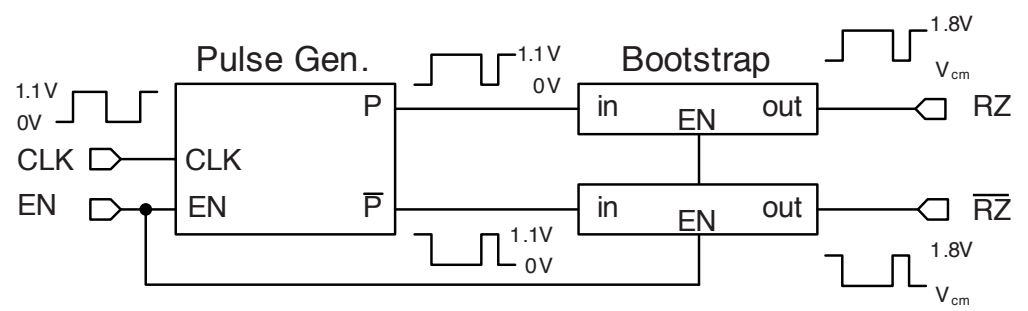

Figure 5.14 - Block-level model of the pulse generator which generates the RZ pulse that drives the error switches.

has two modes of operation. When the ES mode is turned OFF, the $\overline{\mathrm{EN}}$ signal is set to 1 and the outputs of the pulse generator (RZ and $\overline{\mathrm{RZ}}$ set to 0 ), which drive the error switches $\left(M_{1,2,4,5}\right)$, are disabled. During this mode, the input switches $M_{3,6}$ are $\mathrm{ON}$, and error current is directly connected to the input of the first integrator. The switches are scaled such that the non-linear ON resistance does not degrade the linearity of the modulator and the $\overline{\mathrm{EN}}$ signal is bootstrapped to $1.8 \mathrm{~V}$ such that the size of the transistor $M_{3,6}$ chosen can be as small as possible so that the switches do not capacitively load the virtual ground of the integrator.

When the ES mode is turned ON by setting the $\overline{\mathrm{EN}}$ signal to 0 , the error switches $\left(M_{1,2,4,5}\right)$ are driven by the pulse generator (Section 5.4.2). The pulse generator is clocked at $2 \mathrm{GHz}$ and its outputs are bootstrapped to $1.8 \mathrm{~V}$ to minimize the ON resistance of the switches. At the rising edge of RZ, the input stage of the loop filter starts integrating the error current, and at the falling edge of RZ, the input stage of the loop filter completes the integration and holds its output value. At the same time, the input signal and output of the DAC are connected to CM by the switches $M_{1,2}$. The input $\mathrm{CM}$ of the modulator is controlled externally by the input signal, therefore the DAC output is not regulated by a CM stabilization circuit.

\subsubsection{Pulse Generator}

Figure 5.14 shows the block-level diagram of the pulse generator, which consists of a pulse generator core and bootstrap circuit. The pulse generator core creates very short pulses which are synchronized to the rising edge of the sampling clock of the CT $\Delta \Sigma$ modulator. The bootstrap circuitry level shifts the output of the pulse generator from $(0-1.1 \mathrm{~V})$ to $\left(V_{C M}-1.8 \mathrm{~V}\right)$ such that the ON resistance of the error switches is negligible compared to the input resistors. 


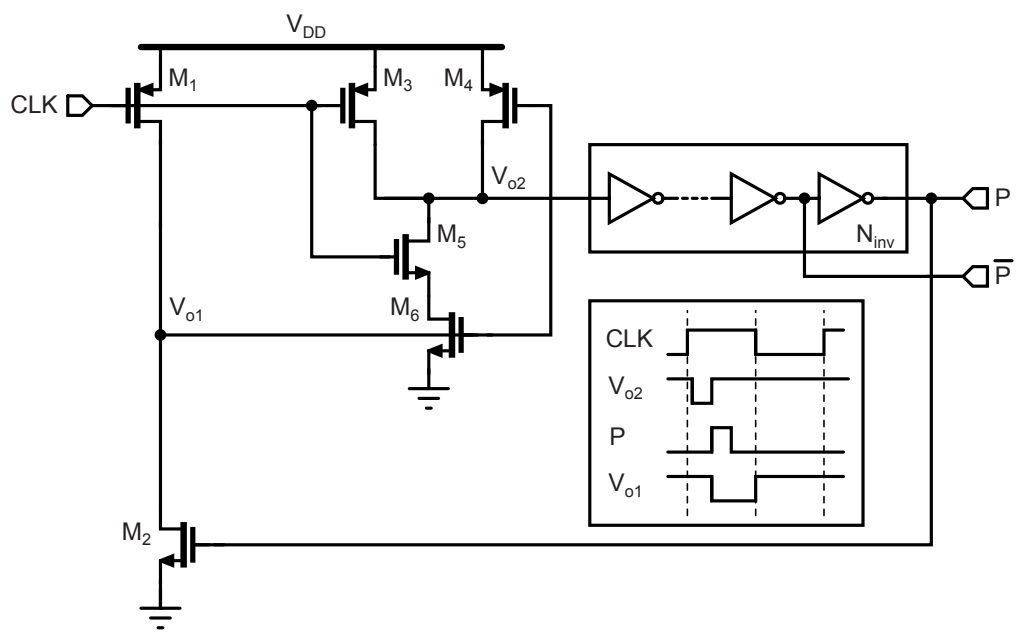

Figure $\mathbf{5 . 1 5}$ - The pulse generator schematic.

Figure 5.15 shows the schematic of the edge triggered monostable pulse generator. The circuit stays at this state until the rising edge of the clock arrives, which causes the circuit to enter the unstable state. The circuit will return to the stable state after a set time which is defined by propagation delay of the inverter chain, where there exists odd number of inverters $\left(N_{\text {inv }}\right)$. The signal diagram presented in Fig. 5.15 shows the critical node voltages of the pulse generator. In the stable state, its output $(\mathrm{P})$ is set to 0 , and internal nodes $\mathrm{V}_{o 1}$ and $\mathrm{V}_{o 2}$ are set to 1 .

The unstable state is triggered by the rising edge of the CLK, at which point the $\mathrm{V}_{o 2}$ discharges to 0 . After the signal propagates through the inverter chain, the output $(\mathrm{P})$ is set to 1 , which drives the gate of $M_{2}$ and sets $\mathrm{V}_{o 1}$ to 0 . As a result, $\mathrm{V}_{o 2}$ is pulled back to $V_{D D}$ and the circuit enters its stable state again. The setup time must be shorter than the clock period. The implemented pulse width is $75 \mathrm{psec}$, which is $0.15 \%$ of the sampling period $\left(T_{s}\right)$. The transistors $\left(M_{1-6}\right)$ and the inverter chain are scaled such that the input-referred root-mean-square (RMS) clock jitter of the modulator meets the target jitter specification when clocked at $2 \mathrm{GHz}$. The pulse generator delivers complementary outputs such that two bootstrapping circuits generate the complementary RZ pulses that drive the error switches.

Figure 5.16 shows the schematic of the bootstrap circuit. $M_{1,2}$ and $C_{1,2}$ form a clock multiplier. At the start up of the circuit, when the IN is $0, M_{2}$ is turned OFF. The bottom plate of $C_{2}$ is 1 , which turns $M_{1}$ ON and charges 


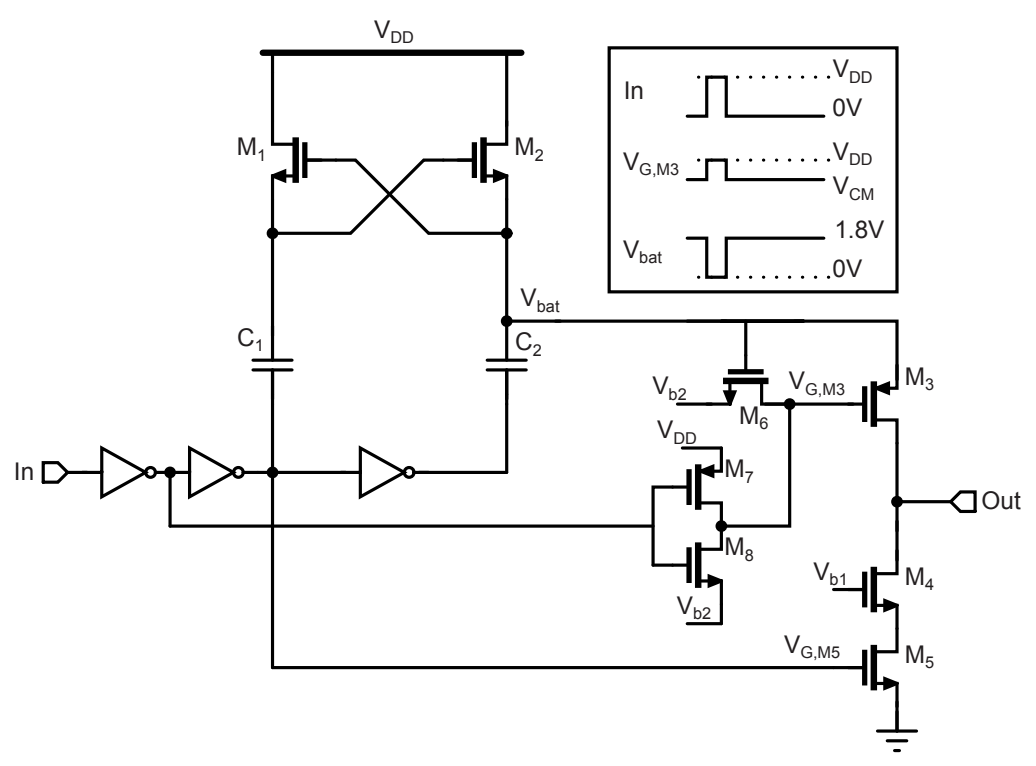

Figure 5.16 - The bootstrap schematic.

$C_{1}$ to $V_{D D}$. In the next phase, the roles are changed and the gate of the $M_{2}$ is pushed up to $2 V_{D D}$, which turns $M_{2} \mathrm{ON}$, and $C_{2}$ is charged to $V_{D D}$. The clock multiplier generates double the supply voltage $\left(V_{D D}=1.1 \mathrm{~V}\right)$ at its output $\left(V_{b a t}\right)$. However, due to the parasitic capacitances, $V_{b a t}$ is limited to $1.8 \mathrm{~V}$, which is good enough in our application. $M_{2}$ and $C_{2}$ must deliver the required power to the load $\left(M_{3-6}\right)$ and must be scaled based on the load. The upper plate of $C_{1}$ drives the gate capacitance of $M_{2}$, which requires much less power than the load. Therefore, $M_{1}$ and $C_{1}$ are scaled smaller than $M_{2}$ and $C_{2}$.

The signal diagram presented in Fig. 5.16 shows the critical node voltages of the bootstrap circuit. Since, the supply voltage of the output stage is boosted to $1.8 \mathrm{~V}$, the circuit is carefully designed such that none of the devices experience relative terminal voltage greater than $V_{D D} . M_{5}$ is protected by the cascode $M_{4}$ and the gate of the $M_{3}$ is driven such that its relative terminal voltages do not exceed $V_{D D}$. The designed pulse generator and bootstrapping circuit generate 75 psec pulses that drive the errors switches, while drawing $10 \mathrm{~mA}$ from a $1.1 \mathrm{~V}$ supply at $2 \mathrm{GHz}$. When disabled, the circuit does not draw any current from the supply. The active area of the layout is negligible when compared to the original size of the chip area. 


\subsection{Experimental Results}

The details of the measurement setup used to evaluate the ADC has been described in Section 4.3.1. The measurements presented in this section aim to verify the effectiveness of the error switching (ES) technique. The layout of the pulse generator and bootstrap circuit is placed between DAC1 and loop filter and the error switches are placed at the virtual ground node of the first integrator. The signals that drive the error signals are routed differentially, isolated from the traces that deliver DAC1 currents. The ADC is clocked at $2 \mathrm{GHz}$, and the signal $\mathrm{BW}$ of the modulator is $62.5 \mathrm{MHz}$. Three samples are measured to evaluate the performance of the ES technique.

Figure 5.17 shows an FFT of the measured-decimated output of the $\Delta \Sigma$ $\mathrm{ADC}$ with no input signal. The noise floor of the ADC is flat in the signal BW of $62.5 \mathrm{MHz}$ and rises slightly at higher frequencies due to the presence of outof-band quantization noise. To measure the distortion of the ADC, sinusoidal input signals with a maximum input voltage of a $2.0-\mathrm{V}_{\mathrm{p}-\mathrm{p}}$ differential are supplied to the ADC. The decimated output for an $2.5 \mathrm{MHz}$ input signal at $-1 \mathrm{dBFS}$ has been captured in real-time, and its FFT is shown in Fig. 5.17. Without the ES technique, the THD is $-68.6 \mathrm{dBc}$ and the peak SNR/SNDR are $66.4 / 64.6 \mathrm{dBc}$, respectively. After the ES technique, both the THD and the peak SNR/SNDR performance of the modulator improve to $-70.1 / 69.2 / 66.8 \mathrm{dBc}$. Therefore, the ES technique improves the THD performance of the modulator,

Figure 5.18 shows an FFT of the measured-decimated output of the $\Delta \Sigma$ $\mathrm{ADC}$ for a $18.5 \mathrm{MHz}$ input signal at $-1 \mathrm{dBFS}$. Without the ES technique, the THD is $-71.2 \mathrm{dBc}$ and the peak SNR/SNDR are $67.1 / 65.7 \mathrm{dBc}$, respectively. For large input signals, the static and dynamic errors of DAC1 causes harmonic components and quantization errors to fold into the signal band and increases the in-band noise. As shown in Fig. 5.18, when the ES mode is turned ON, the $\mathrm{HD}_{2} / \mathrm{HD}_{3}$ tones reduce from $-76.7 /-72.6 \mathrm{dBc}$ to $-84.6 /-77.9 \mathrm{dBc}$, respectively. The ES technique masks the dynamic errors and improves the THD performance of the modulator. More importantly, the noise performance of the modulator improves. After turning on the ES technique, the peak SNR/SNDR increases to $69.4 / 68.7 \mathrm{dBc}$. Therefore, the ES technique improves the THD performance of the modulator, and also reduces the high-frequency quantization noise which folds into the signal band due to the non-linearity of DAC1. There is especially more improvement in THD when compared to the case where an $2.5 \mathrm{MHz}$ input signal is applied. The dynamic errors scale with input frequency and becomes more dominant than the static errors for high frequency input signals.

Table 5.1 summarizes the performance of ES technique for three samples 


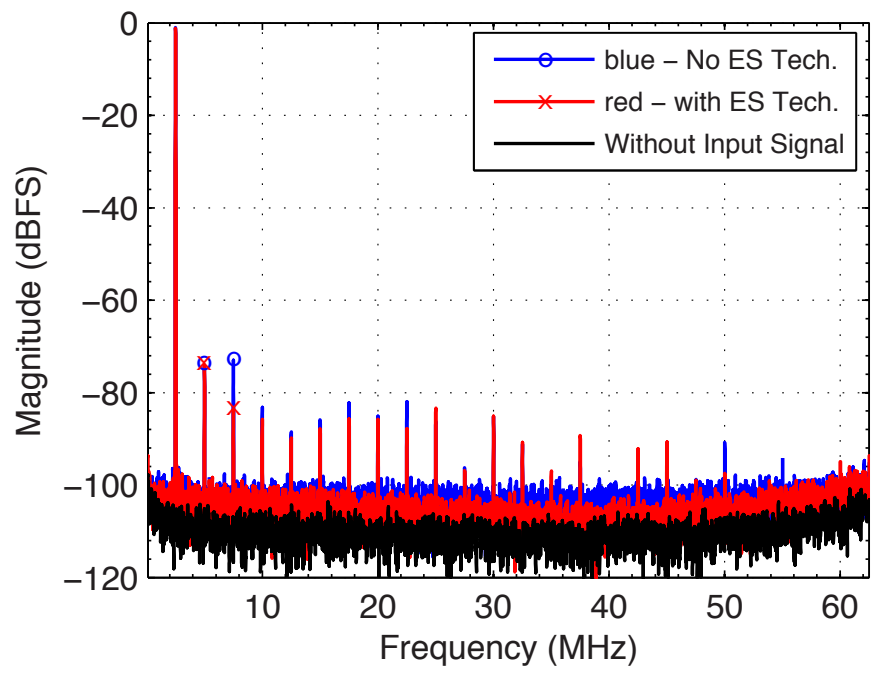

Figure 5.17 - An FFT of the measured decimated output for a single-tone input signal of $-1 \mathrm{dBFS}$ at $2.5 \mathrm{MHz}$. RBW is $6.1 \mathrm{KHz}$. (Sample-1)

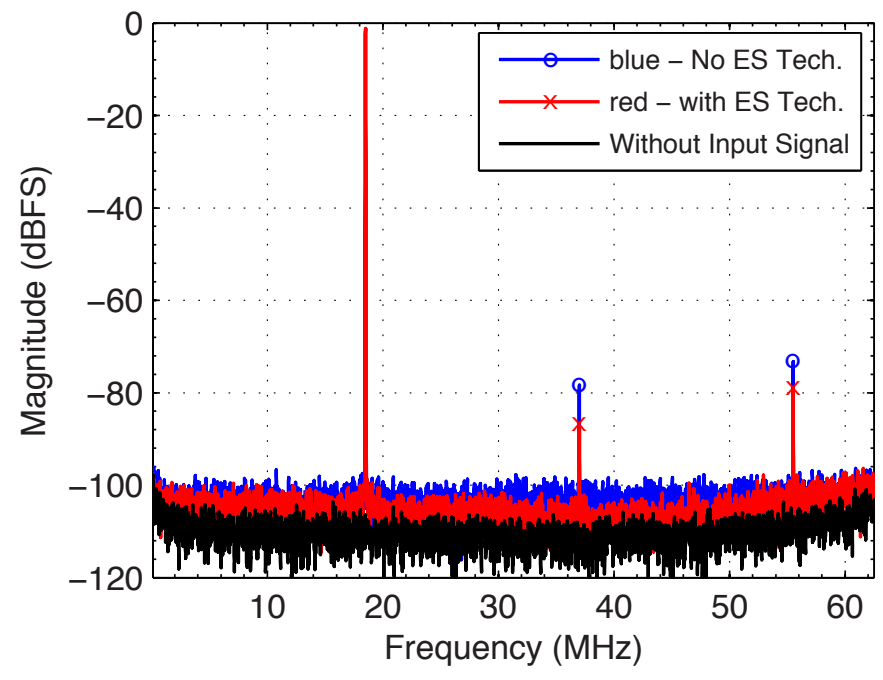

Figure 5.18 - An FFT of the measured decimated output for a single-tone input signal of $-1 \mathrm{dBFS}$ at $18.5 \mathrm{MHz}$. RBW is $6.1 \mathrm{KHz}$. (Sample-1) 
Table 5.1 - Performance summary of the measured samples before and after the error switching (ES) technique for an $18.5 \mathrm{MHz}$ input signal at $-1 \mathrm{dBFS}$.

\begin{tabular}{|c|c|c|c|c|}
\hline Sample & Specification $(\mathrm{dBc})$ & Before ES & After ES & Improvement $(\mathrm{dB})$ \\
\hline \multirow{3}{*}{ No. 1 } & $\mathrm{SNR}$ & 67.1 & 69.4 & 2.3 \\
& $\mathrm{SNDR}$ & 65.7 & 68.7 & 3.0 \\
& $\mathrm{THD}$ & -71.2 & -77.0 & 5.8 \\
& $\mathrm{HD}_{2}$ & -76.7 & -84.6 & 7.9 \\
& $\mathrm{HD}_{3}$ & -72.6 & -77.9 & 5.2 \\
\hline \multirow{3}{*}{ No. 2} & $\mathrm{SNR}$ & 66.6 & 68.6 & 2.0 \\
& $\mathrm{SNDR}$ & 62.1 & 65.9 & 3.8 \\
& $\mathrm{THD}$ & -64.0 & -69.3 & 5.3 \\
& $\mathrm{HD}$ & -81.5 & -90.5 & 9.0 \\
& $\mathrm{HD}$ & -64.1 & -69.4 & 5.3 \\
\hline \multirow{3}{*}{ No.3 } & $\mathrm{SNR}^{3}$ & 68.7 & 70.2 & 1.5 \\
& $\mathrm{SNDR}^{*}$ & 66.9 & 69.6 & 2.7 \\
& $\mathrm{THD}$ & -71.7 & -78.9 & 7.2 \\
& $\mathrm{HD}_{2}$ & -80.3 & -79.1 & -1.2 \\
& $\mathrm{HD}_{3}$ & -72.4 & -91.4 & 19.0 \\
\hline
\end{tabular}


that are evaluated for an $18.5 \mathrm{MHz}$ input signal at $-1 \mathrm{dBFS}$. The measured THD, SNR, and SNDR improved after applying the ES technique. On the other hand, we should notice that the $\mathrm{HD}_{2}$ of sample three degraded after applying the ES technique, which is a possible outcome of the experiment since the HD performance of some of the samples is dominated by the static errors. For sample three, we may conclude that the $\mathrm{HD}_{2}$ was mainly dominated by the static errors. Since, the static errors are not calibrated in this test chip, we are not able to identify the root cause of the $\mathrm{HD}_{2}$, which requires further research.

The circuits used for the ES technique only increases the power consumption of the modulator by $11 \mathrm{~mW}$, yet especially the SNDR of the modulator improves approximately $3 \mathrm{~dB}$ based on the measurements listed in Table 5.1. The ES technique improves the figure of merit (FoM) of the ADC by approximately $25 \%$, which is very important for applications targeting high performance and low power at the same time.

\subsection{Conclusions}

The performance of a high-speed multi-bit $\mathrm{CT} \Delta \Sigma \mathrm{ADC}$ is limited by the static and dynamic errors of its feedback DAC. There are various mechanisms behind the static errors and dynamic errors some of which can be corrected and calibrated by use of auxiliary circuits at the cost of increasing the complexity of the ADC. This work demonstrates the implementation of a multi-bit $2 \mathrm{GHz}$ $\mathrm{CT} \Delta \Sigma \mathrm{ADC}$ with an error switching (ES) scheme applied to the virtual ground node of the first integrator. By applying the ES technique, the contribution of the dynamic errors are tested and it is shown that this technique improves the SNR, SNDR of the modulator but also the THD, which are all dominated by the dynamic errors. Furthermore, the power efficiency of the modulator improves $25 \%$ and the ES technique demonstrates that the dynamic performance of oversampled converters implemented in nm-CMOS can achieve the state-of-the-art performance.

\section{References}

[1] K. Doris, A. H. v. Roermund, and D. Leenaerts, Wide-Bandwidth High Dynamic Range D/A Converters. Springer, 2006.

[2] T. Yongjian, H. Hegt, and A. van Roermund, Dynamic-Mismatch Mapping for Digitally-Assisted DACs. Springer, 2013. 
[3] C.-H. Lin et al., "A 12 bit $2.9 \mathrm{GS} / \mathrm{s}$ DAC with $\mathrm{IM} 3<-60 \mathrm{dBc}$ beyond 1 GHz in 65 nm CMOS," in Solid-State Circuits, IEEE Journal of, vol. 44, no. 12, pp. 3285-3293, Dec. 2009.

[4] B. Schafferer and R. Adams, "A 3V CMOS 400mW 14b 1.4GS/s DAC for multi-carrier applications," in Solid-State Circuits Conference, 2004. Digest of Technical Papers. ISSCC. 2004 IEEE International, Feb. 2004, pp. 360-532 Vol.1.

[5] A. van den Bosch, M. Borremans, M. Steyaert, and W. Sansen, "A 10-bit 1-GSample/s Nyquist current-steering CMOS D/A converter," in SolidState Circuits, IEEE Journal of, vol. 36, no. 3, pp. 315-324, Mar. 2001.

[6] P. Drennan and C. McAndrew, "Understanding MOSFET mismatch for analog design," in Solid-State Circuits, IEEE Journal of, vol. 38, no. 3, pp. 450 - 456, Mar. 2003.

[7] P. Palmers and M. Steyaert, "A $11 \mathrm{~mW}$ 68dB SFDR $100 \mathrm{MHz}$ bandwidth $\Sigma \Delta$-DAC based on a 5 -bit $1 \mathrm{GS} / \mathrm{s}$ core in $130 \mathrm{~nm}, "$ in Solid-State Circuits Conference, 2008. ESSCIRC 2008. 34th European, Sept. 2008, pp. 214217.

[8] W. Schofield, D. Mercer, and L. Onge, "A 16b 400MS/s DAC with <$80 \mathrm{dBc}$ IMD to $300 \mathrm{MHz}$ and $<-160 \mathrm{dBm} / \mathrm{Hz}$ noise power spectral density," in Solid-State Circuits Conference, 2003. Digest of Technical Papers. ISSCC. 2003 IEEE International, 2003, pp. 126-482 vol.1.

[9] R. Baird and T. Fiez, "Improved $\Delta \Sigma$ DAC linearity using data weighted averaging," in Circuits and Systems, 1995. ISCAS '95., 1995 IEEE International Symposium on, vol. 1, Apr. 1995, pp. 13-16 vol.1.

[10] C. Conroy, W. Lane, and M. Moran, "Statistical design techniques for D/A converters," in Solid-State Circuits, IEEE Journal of, vol. 24, no. 4, pp. 1118-1128, Aug. 1989.

[11] T. Chen and G. Gielen, "A 14-bit 200-MHz current-steering DAC with switching-sequence post-adjustment calibration," in Solid-State Circuits, IEEE Journal of, vol. 42, no. 11, pp. 2386-2394, Nov. 2007.

[12] Z. Li and T. Fiez, "A 14 bit continuous-time delta-sigma A/D modulator with $2.5 \mathrm{MHz}$ signal bandwidth," in Solid-State Circuits, IEEE Journal of, vol. 42, no. 9, pp. 1873-1883, Sept. 2007. 
[13] P. Witte, J. Kauffman, J. Becker, Y. Manoli, and M. Ortmanns, "A 72dBDR $\Delta \Sigma$ CT modulator using digitally estimated auxiliary DAC linearization achieving $88 \mathrm{fJ} /$ conv in a $25 \mathrm{MHz}$ BW," in Solid-State Circuits Conference Digest of Technical Papers (ISSCC), 2012 IEEE International, Feb. 2012, pp. 154-156.

[14] K. Doris, J. Briaire, D. Leenaerts, M. Vertreg, and A. van Roermund, "A $12 \mathrm{~b} 500 \mathrm{MS} / \mathrm{s}$ DAC with $>70 \mathrm{~dB}$ SFDR up to $120 \mathrm{MHz}$ in $0.18 \mu \mathrm{m}$ CMOS," in Solid-State Circuits Conference, 2005. Digest of Technical Papers. ISSCC. 2005 IEEE International, Feb. 2005, pp. 116-588 Vol. 1.

[15] L. Risbo et al., "Digital approaches to ISI-mitigation in high-resolution oversampled multi-level D/A converters," in Solid-State Circuits, IEEE Journal of, vol. 46, no. 12, pp. 2892-2903, Dec. 2011.

[16] M. Bolatkale, L. Breems, R. Rutten, and K. Makinwa, "A 4GHz CT $\Delta \Sigma$ ADC with 70dB DR and -74dBFS THD in 125MHz BW ," in Solid-State Circuits Conference, 2011. ISSCC 2011. Digest of Technical Papers. IEEE International, Feb. 2011, pp. 470-472.

[17] H. Shibata et al., "A DC-to- $1 \mathrm{GHz}$ tunable RF $\Delta \Sigma$ ADC achieving $\mathrm{DR}=74 \mathrm{~dB}$ and $\mathrm{BW}=150 \mathrm{MHz}$ at $f_{0}=450 \mathrm{MHz}$ using $550 \mathrm{~mW}$," in SolidState Circuits Conference, 2012. ISSCC 2012. Digest of Technical Papers. IEEE International, Feb. 2012, pp. 150-151.

[18] R. W. Adams, "Design and implementation of an audio 18-bit analog-todigital converter using oversampling techniques," in J. Audio Eng. Soc, vol. 34, no. 3, pp. 153-166, 1986.

[19] E. van der Zwan and E. Dijkmans, "A 0.2-mW CMOS $\Sigma \Delta$ modulator for speech coding with $80 \mathrm{~dB}$ dynamic range," in Solid-State Circuits, IEEE Journal of, vol. 31, no. 12, pp. 1873-1880, Dec. 1996.

[20] S. Park, G. Kim, S.-C. Park, and W. Kim, "A digital-to-analog converter based on differential-quad switching," in Solid-State Circuits, IEEE Journal of, vol. 37, no. 10, pp. 1335-1338, Oct. 2002.

[21] K. Nguyen, R. Adams, K. Sweetland, and H. Chen, "A 106-dB SNR hybrid oversampling analog-to-digital converter for digital audio," in Solid-State Circuits, IEEE Journal of, vol. 40, no. 12, pp. 2408-2415, Dec. 2005. 


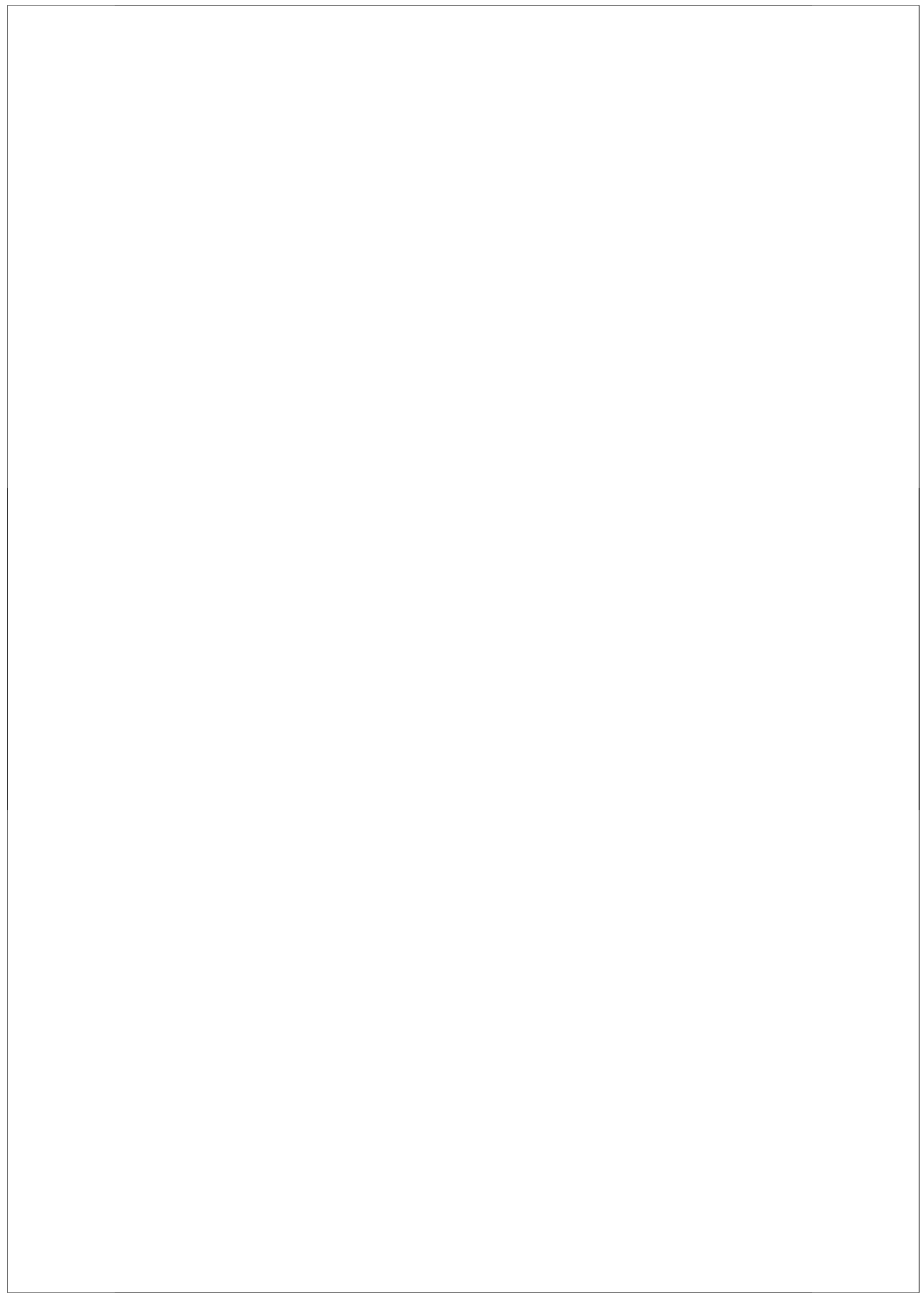




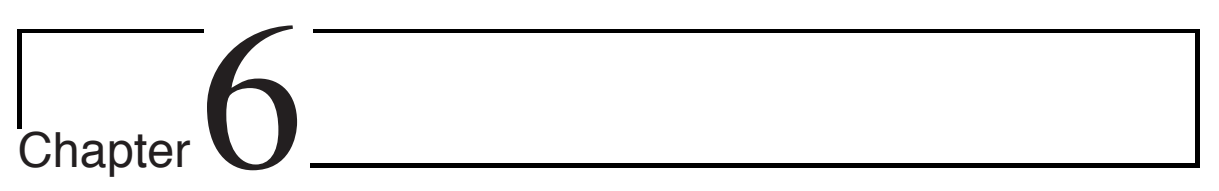

\section{Conclusions}

This work experimentally demonstrates the feasibility and design of a wideband, high-dynamic range oversampled analog-to-digital converter (ADC) that can reach a performance comparable to Nyquist ADCs. Oversampled converters, especially continuous-time delta-sigma (CT $\Delta \Sigma)$ modulators, offer various advantages. Simple resistive input, for instance, does not require the use of a power-hungry input buffer or an anti-aliasing filter, which simplifies and enables system integration. The quest for a wide bandwidth and high dynamic range with an oversampled converter, which is the aim of this work, brings with it questions about the power efficiency of the modulator at a gigahertz sampling frequency and how to formulate the relation between stability and power efficiency.

The multi-bit high-order modulators is one of the most promising architectures that enables a wide bandwidth and high dynamic range $[1,2]$. Due to the oversampling, typically such converters require a high sampling frequency which is in the order of $3-5 \mathrm{GHz}$. The performance of a delta-sigma modulator $(\Delta \Sigma \mathrm{M})$ sampled at a few $\mathrm{GHz}$ is limited by excess loop delay (ELD), phase margin of the loop filter at $f_{s} / 2$, and clock jitter. The ELD and phase margin of the loop filter define the stability of the modulator. The ELD, due to latency of the quantizer, requires an additional feedback path around the quantizer. In the case of common ELD compensation techniques, the stability of the modulator is defined by the additional feedback path which increases the requirements of the summation node. As a result, the sampling speed of the modulator is limited by the performance of a summing amplifier and its high speed feedback path around the quantizer. Secondly, it is very difficult 
to achieve the phase margin of the loop filter at $f_{s} / 2$ especially in the presence of amplifiers with a finite gain-bandwidth product (GBW) and parasitic poles. This can be overcome by using a very high-speed amplifier at the cost of excessive power dissipation. In this work, by implementing a high speed capacitive feedforward loop filter, the fundamental limitations posed by the summing amplifier are solved, and the phase margin of the loop filter at $f_{s} / 2$ no longer depends on the summing amplifier.

In addition to the stability criteria of a $\Delta \Sigma \mathrm{M}$, its maximum dynamic range is defined by its sensitivity to white noise jitter. In the presence of a large input signal, the spectral shape of jitter noise limits the achievable signal-to-noise ratio (SNR) and dynamic range (DR). Therefore, the noise contribution of the clock buffers must be optimized to meet the phase-noise requirements. Using a multi-bit quantizer relaxes the jitter requirement. However, the load impedance of the clock buffers increases as the resolution of the digital-to-analog converter (DAC) increases as does the power consumption of the clock circuitry. At a $\mathrm{GHz}$ sampling frequency, the clock circuitry is one of the building block of the modulator dominating the power consumption.

\subsection{Benchmarking}

The first outcome of this work is the design of a high-speed $\Delta \Sigma \mathrm{M}$ that overcomes limitations listed in the previous section and enables $\mathrm{GHz}$ sampling rates with state-of-the-art power efficiency. Table 6.1 summarizes the performance of the ADC. The $4 \mathrm{GHz}$ CT $\Delta \Sigma$ ADC is implemented in $45 \mathrm{~nm}-\mathrm{LP}$ CMOS process and achieves 70dB DR and -74dBFS total harmonic distortion (THD) in a $125 \mathrm{MHz}$ bandwidth. Compared to the CMOS $\Delta \Sigma$ ADC presented in [3], the proposed ADC achieves a $5 \mathrm{x}$ larger bandwidth (BW) with a similar dynamic range. Compared to the Nyquist ADC presented in [4], the proposed ADC achieves a similar BW but one-bit less dynamic range. However, the pipeline ADC uses a technology with slower transistors. A Nyquist ADC which can be implemented in the same process node (45nm CMOS) is capable of achieving wider bandwidth but, the accuracy will be limited by the DAC matching and the $k T / C$ thermal noise. Similarly, the accuracy of the implemented ADC is also limited by the matching of its multi-bit DAC and thermal noise. However, its DR can be improved by reducing its effective input-referred noise resistance. This will be at the expense of increased power consumption in the first integrator, which, however, contributes only $10 \%$ of the ADC's total power dissipation. The multi-bit DAC relaxes the jitter requirement of the ADC, while the jitter of the clock which drives the sample-and-hold of a Nyquist ADC limits its max- 
Table 6.1 - Performance summary and comparison to prior work published before 2012 .

\begin{tabular}{|l|c|c|c|}
\hline & $\begin{array}{c}\text { This Work } \\
\text { ISSCC'11 }\end{array}$ & $\begin{array}{c}\text { Kauffman [3] } \\
\text { ISSCC'11 }\end{array}$ & $\begin{array}{c}\text { Ali [4] } \\
\text { JSSC'10 }\end{array}$ \\
\hline Architecture & $\Delta \Sigma$ & $\Delta \Sigma$ & Pipeline \\
\hline$f_{s}(\mathrm{GHz})$ & 4 & 0.5 & 0.25 \\
\hline BW $(\mathrm{MHz})$ & 125 & 25 & 125 \\
\hline DR $(\mathrm{dB})$ & 70 & 70 & 77.5 \\
\hline SNR $(\mathrm{dB})$ & 65.5 & 64 & 77.5 \\
\hline SNDR $(\mathrm{dB})$ & 65 & 63.5 & 77.5 \\
\hline Power $(\mathrm{mW})$ & 259.2 & 8 & 1000 \\
\hline$V_{\text {DD }}(\mathrm{V})$ & $1.1 / 1.8$ & 1.2 & $1.8 / 3.0$ \\
\hline Area $\left(\mathrm{mm}^{2}\right)$ & 0.9 & 0.15 & 50 \\
\hline Technology & $45 \mathrm{~nm}$ & $90 \mathrm{~nm}$ & $180 \mathrm{~nm}$ \\
& CMOS & CMOS & BiCMOS \\
\hline FoM $(\mathrm{pJ} / \text { conv.-step })^{a}$ & $0.71^{c}$ & 0.13 & 0.55 \\
\hline FoM $(\mathrm{pJ} / \text { conv.-step })^{b}$ & $0.40^{c}$ & 0.06 & 0.55 \\
\hline
\end{tabular}

${ }^{a} \mathrm{FoM}=$ Power $/\left(2 \times \mathrm{BW} \times 2^{(\mathrm{SND} R-1.76) / 6.02}\right)$.

${ }^{b} \mathrm{FoM}=$ Power $/\left(2 \times \mathrm{BW} \times 2^{(\mathrm{DR}-1.76) / 6.02}\right)$.

${ }^{c}$ The power consumption of the decimation filter is included in the FoM.

imum achievable DR. The proposed ADC has a better figure of merit (FoM) (based on DR) than the Nyquist ADC [4] which implies that $\Delta \Sigma$ ADCs can be a power-efficient alternative for applications which require a high dynamic range and wide BWs. Most of the power in the proposed ADC is dissipated in digital circuits and for each new generation of the CMOS process we can expect a power efficiency improvement. On the other hand, the power consumption of a pipeline ADC is limited by its interstage gain amplifiers. Moreover, the power dissipation of noise limited pipeline ADCs will not reduce with a new generation of CMOS technology [5].

The performance of multi-bit GHz CT $\Delta \Sigma$ ADCs is limited by the static and dynamic errors of its feedback DAC. The static errors can be calibrated, which improves the low-frequency performance of the modulators. In this work, at $\mathrm{GHz}$ sampling rates, we have demonstrated that the dynamic errors limit the performance of the modulator and that these errors can be corrected by using the error switching (ES) scheme. The second outcome of this work is the implementation of a multi-mode version of the multi-bit $\mathrm{CT} \Delta \Sigma \mathrm{ADC}$, which is sampled at $2 \mathrm{GHz}$. A power-efficient dynamic error correction technique has been implemented which improves the THD, SNR, SNDR performance of the 
modulator. The dynamic error correction technique improves the FoM by $25 \%$.

\subsection{Future Work}

The future work suggestions are based on the insight gained during this research. High-speed ADC developments are driven by two main factors. The first is the increasing demand for signal bandwidth and dynamic range in applications such as wireline and wireless communications which pushes the performance requirements. Secondly, the power consumption of the input/output (I/O) circuitry cannot be neglected at $\mathrm{GHz}$ sampling frequencies. As the complexity of the system increases, more power is dissipated to drive and distribute $\mathrm{I} / \mathrm{O}$ signals. The I/O circuitry of the ADC also benefits greatly from the increasing $f_{T}$ of every new generation of nm-CMOS technology.

The possible future research directions in $\mathrm{CT} \Delta \Sigma$ modulators can be clustered into three categories:

\section{Power Efficiency}

The presented ADC in this work achieves a FoM of $0.7 \mathrm{pJ} /$ conv.-step, when sampled at $4 \mathrm{GHz}$. The low-latency digital circuits and low-noise clock buffers consume most of the power. It would be of great interest if the FoM of the ADC can be improved at least by an order of magnitude while keeping the same specifications. To achieve this target, newer process technologies will help to reduce the digital power consumption of the ADC. The thermal noise requirement of the loop filter and the feedback DAC sets a lower boundary for the power dissipation. However, innovative modulator/circuit architectures which focus on reducing the dynamic power dissipation are still required to design a stable modulator with better power efficiency.

\section{Linearity beyond $80 \mathrm{dBc}$ and Blocker Suppression}

A multi-bit $\Delta \Sigma \mathrm{M}$, if not calibrated/corrected, suffers from non-linearity which is usually limited by the dynamic and static errors of its multi-bit DAC, quantizer, and ELD DAC. To overcome these limitations and achieve a spuriousfree dynamic range (SFDR) better than $80 \mathrm{dBc}$ is a major research challenge. It requires an understanding of the sources of non-linearity and the calibration techniques that are suitable for high-frequency sampling. Furthermore, the calibration/correction techniques should not degrade the stability of the modulator. In order to achieve an SFDR better than $80 \mathrm{dBc}$, the static errors must be calibrated and the coupling effects at $\mathrm{GHz}$ sampling rates must be 
investigated. This research challenge can be further studied in the presence of blockers which are amplified when the signal transfer function (STF) of the modulator peaks outside the signal bandwidth. A single-bit modulator does not suffer from the non-linear multi-bit DAC, but it does require a very highspeed sampling clock probably greater than $f_{s}>10 \mathrm{GHz}$ in order to achieve a signal bandwidth greater than $125 \mathrm{MHz}$. At these sampling frequencies, the signal-dependent delay of the digital circuits might set a fundamental limit on the linearity of the modulator. However, this limitation is relaxed for every new generation of nm-CMOS technology.

\section{Ultra Wideband and High Dynamic Range Oversampled Converters}

One of the boundaries which limits the bandwidth of an oversampled ADC is the maximum achievable switching speed of the digital circuits and latches. Increasing the bandwidth of this modulator an order of magnitude with a similar dynamic range is definitely an interesting research challenge. The switching speed $\left(f_{T}\right)$ of every new generation of nm-CMOS technology only improves $10-20 \%$, from which we can assume that the signal bandwidth of oversampled converters will follow the increasing $f_{T}$ of the technology. Therefore, innovative modulator architectures and digital circuit design techniques are required to achieve $10 \times$ more bandwidth in the near future. Although ELD, phase margin of the loop filter at $f_{s} / 2$, and clock jitter will be the major limiting factors, the outcome of the research results will be very valuable.

\section{References}

[1] M. Bolatkale, L. Breems, R. Rutten, and K. Makinwa, "A $4 \mathrm{GHz}$ continuous-time $\Delta \Sigma$ ADC with $70 \mathrm{~dB}$ DR and $-74 \mathrm{dBFS}$ THD in 125 MHz BW," in Solid-State Circuits, IEEE Journal of, vol. 46, no. 12, pp. 2857-2868, Dec. 2011.

[2] H. Shibata et al., "A DC-to- $1 \mathrm{GHz}$ tunable RF $\Delta \Sigma$ ADC achieving $\mathrm{DR}=74 \mathrm{~dB}$ and $\mathrm{BW}=150 \mathrm{MHz}$ at $f_{0}=450 \mathrm{MHz}$ using $550 \mathrm{~mW}$," in Solid-State Circuits Conference, 2012. ISSCC 2012. Digest of Technical Papers. IEEE International, Feb. 2012, pp. 150-151.

[3] J. Kauffman, P. Witte, J. Becker, and M. Ortmanns, "An 8mW 50MS/s $\mathrm{CT} \Delta \Sigma$ modulator with $81 \mathrm{~dB}$ SFDR and digital background DAC linearization," in Solid-State Circuits Conference Digest of Technical Papers (ISSCC), 2011 IEEE International, Feb. 2011, pp. 472-474. 
[4] A. Ali et al., "A 16-bit 250-MS/s IF sampling pipelined ADC With background calibration," in Solid-State Circuits, IEEE Journal of, vol. 45, no. 12, pp. 2602-2612, Dec. 2010.

[5] T. Sundstrom, B. Murmann, and C. Svensson, "Power dissipation bounds for high-speed nyquist analog-to-digital converters," Circuits and Systems I: Regular Papers, IEEE Transactions on, vol. 56, no. 3, pp. 509-518, 2009. 


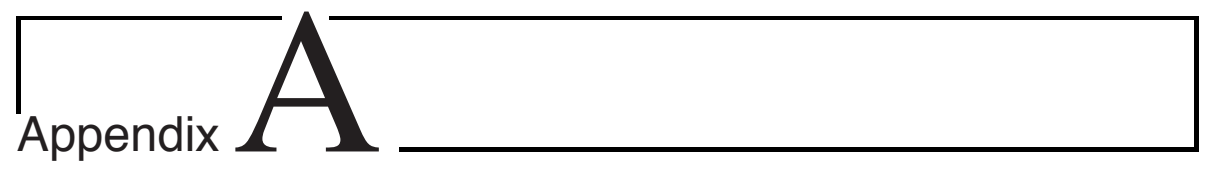

\section{Comparison of ADC Architectures}

A comparison of continuous-time delta-sigma $(\mathrm{CT} \Delta \Sigma)$, pipeline, and timeinterleaved (TI) SAR ADCs which target wide signal bandwidths (greater than $100 \mathrm{MHz}$ ) and high dynamic ranges (more than 12-bit) is presented in Table A.1. In this comparison, we assume that ADCs are thermal noise limited. The comparison presented in this section only covers top level design choices. The reference circuits and clocking overhead required by each architecture are neglected.

The first section of the table focuses on the system level requirements. Compared to Nyquist ADCs, $\Delta \Sigma$ modulators require higher sampling clock $\left(f_{s}\right)$ due to the required oversampling ratio. The input network is often implemented with a simple resistive input, which is the most important advantage of $\mathrm{CT} \Delta \Sigma$ modulators compared to Nyquist converters that require an input sampling network often implemented with a switched-capacitor network. The resistive input relaxes the requirements of an input buffer, because it is much easier to drive a resistive load than a switch capacitor load that requires high peak currents. In order to implement all these ADCs, certain blocks are required as summarized in Table A.1. CT $\Delta \Sigma$ ADCs require a high speed clock source and a decimation filter which is fundamentally different from Nyquist converters. Because of the decimation filter, which is needed to suppress the out-of-band quantization noise, $\Delta \Sigma$ modulators have the highest latency. Pipeline converters require $K$-stages to convert the signal into digital, therefore their latency is proportional to the number of stages. On the other hand, SAR convert- 
ers have the smallest latency. In applications where latency requirement for a given resolution is critical, special attention must be paid to the choice of ADC architecture. The settling requirement of the buffer that drives the ADC is not included in this comparison. Assuming that for each architecture, the bandwidth of the clock network is set to its sampling frequency, $\mathrm{CT} \Delta \Sigma$ modulators with single-bit DAC have the most stringent jitter requirement. Moreover, the white noise of the oscillator mixes with the out-of-band quantization noise and down-converts it into the baseband.

The second section of the table focuses on the design requirements of the ADC architectures. The input network is one of the biggest contributor to the thermal noise. CT $\Delta \Sigma$ modulators require a resistor, which can be implemented using much smaller area compared to an input sampling capacitor which is limited by thermal noise specification. The number of comparators required by each architecture varies, but the important design requirement is the sampling speed of each comparator. We assume that comparators used in each architecture have the same time constant $\left(\tau_{c o m p}\right)$. For a TI-SAR ADC, if we assume that the number of time-interleaved slices $(L)$ is much greater than the resolution of the $\operatorname{ADC}(N)$, then it has the smallest comparator sampling rate requirement. BER of a comparator decreases exponentially with $\left(\propto T_{s} / \tau_{\text {comp }}\right)$ where $T_{s}$ is the sampling period of the comparator. On the other hand, $\Delta \Sigma$ modulator has the most stringent BER requirement due to is high sampling rate.

The sampling speed of a $\Delta \Sigma$ modulator is defined by the latency of its comparator. The sampling speed of the SAR converter is limited by latency of its comparator and DAC settling, where as the sampling speed of a pipeline converter is limited by the settling of its inter-stage gain amplifier. In order to achieve resolution higher than 10-bits, both pipeline and SAR converters require calibration. However, if a single-bit $\Delta \Sigma$ modulator is employed, a high resolution converter can be designed without calibration. The power dissipation of the $\Delta \Sigma$ modulator is limited by the digital circuits that are clocked at the sampling speed. On the other hand, the pipeline converter requires a power hungry amplifier in its first stage. The SAR converter's comparator, which is designed to meet the noise and speed requirement, often dominates the power dissipation.

The last section of the table briefly presents the impact of technology on the choice of architecture. In general, all the architectures benefit from the high $f_{T}$ of the technology, which increases the sampling speed of comparators and the unity gain bandwidth (UGBW) of amplifiers. However, the limited intrinsic gain of nm-CMOS technology with low supply voltage increases the effort required to design amplifiers that can be used in $\Delta \Sigma$ and pipeline converters. 
Table A.1 - Comparison of ADC architectures targeting wide bandwidth (BW> $100 \mathrm{MHz})$ and high dynamic range (DR $>70 \mathrm{~dB})$.

\begin{tabular}{|c|c|c|c|}
\hline & \multicolumn{3}{|c|}{ ADC Architecture } \\
\hline & \multirow{2}{*}{$\begin{array}{c}\text { Oversampled } \\
\mathrm{CT} \Delta \Sigma \\
\left(M^{t h} \text {-order, } B \text {-bit }\right)\end{array}$} & \multicolumn{2}{|c|}{ Nyquist } \\
\hline & & $\begin{array}{c}\text { Pipeline } \\
(K \text {-stages })\end{array}$ & $\begin{array}{c}\text { TI-SAR } \\
(L \text {-times })\end{array}$ \\
\hline \multicolumn{4}{|c|}{ System level requirement } \\
\hline Sampling rate $\left(f_{s}\right)$ & $O S R \cdot f_{N Q}$ & $f_{N Q}$ & $f_{N Q}$ \\
\hline Anti-aliasing & + & - & - \\
\hline Input buffer & + & - & - \\
\hline Required blocks & $\begin{array}{c}\text { Amplifier } \\
\text { Comparator } \\
\text { DAC } \\
\text { High-speed clock } \\
\text { Decimation filter }\end{array}$ & $\begin{array}{c}\text { Sample-and-hold } \\
\text { Amplifier } \\
\text { Comparator } \\
\text { DAC }\end{array}$ & $\begin{array}{c}\text { Sample-and-hold } \\
\text { Comparator } \\
\text { DAC }\end{array}$ \\
\hline Latency & -- & - & + \\
\hline Jitter & $\begin{array}{l}-(B=1) \\
+(B>1)\end{array}$ & $\begin{array}{l}\mathrm{O} \\
\mathrm{o}\end{array}$ & $\begin{array}{l}\mathrm{O} \\
\mathrm{O}\end{array}$ \\
\hline \multicolumn{4}{|l|}{ Design requirement } \\
\hline Input network noise & $4 k T R_{\text {in }}$ & $k T / C$ & $k T / C$ \\
\hline Number of comparators & $2^{B}-1$ & $>K$ & $>L$ \\
\hline Comparator speed & $f_{\Delta \Sigma}=O S R \cdot f_{N Q}$ & $f_{\text {pipe }}=f_{N Q}$ & $f_{S A R} \geq(N / L) \cdot f_{N Q}{ }^{1}$ \\
\hline Bit-error-ratio (BER) & -- & + & $\begin{array}{c}++(N / L<1) \\
-(N / L \geq 1)\end{array}$ \\
\hline Speed is limited by & Comparator latency & Amplifier settling & $\begin{array}{c}\text { Comparator latency } \\
\text { DAC settling }\end{array}$ \\
\hline Number of amplifiers & $<M$ & $<K$ & - \\
\hline Power is limited by & $\begin{array}{l}\text { High-speed digital } \\
\text { Clock }\end{array}$ & Amplifier & Comparator \\
\hline Calibration & $\begin{array}{l}+(B=1) \\
-(B>1)\end{array}$ & - & - \\
\hline \multicolumn{4}{|l|}{ Technology } \\
\hline High $f_{T}$ & + & + & + \\
\hline Limited gain $\left(g_{m} \cdot r_{\text {out }}\right)$ & - & - & $\mathrm{O}$ \\
\hline Area & - & -- & + \\
\hline
\end{tabular}

${ }^{1} N$ is the resolution of the $\mathrm{ADC}$ 
Ideally, SAR converters should not employ any amplifier, however, in order to drive the different TI-slices, some designs might use buffers. Finally, SAR converters have the potential to achieve the smallest area, since they only require capacitors and a comparator. On the other hand, $\Delta \Sigma$ modulators and pipeline converters employ amplifiers. Assuming that $K>M$, pipeline converters have larger area than $\Delta \Sigma$ modulators. 


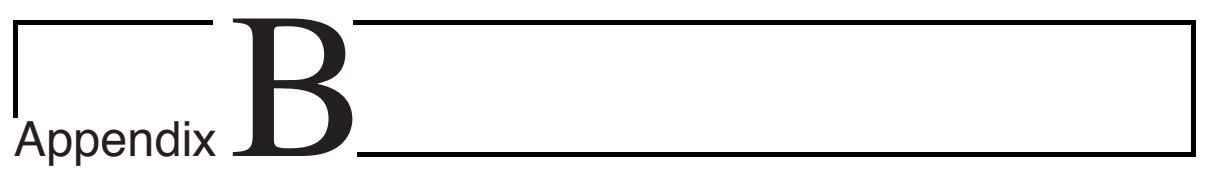

\section{Non-linearity of an Ideal Quantizer}

In order to analyze the non-linearity of an ideal quantizer, we follow the approach presented by Blachman [1]. Throughout the analysis, we assume that the quantizer has a unit step size. A B-bit quantizer with a unit step size has a gain one, and its output waveform can be expressed as the sum of the input signal plus a periodic saw-tooth wave. To analyze the effect of amplitude quantization, the sawtooth wave can be expressed in fourier series [1]:

$$
y(x)=x+\sum_{n=1}^{\infty} \frac{\sin (2 n \pi x)}{n \pi} .
$$

For a sine-wave input $(x(t)=A(t) \sin (\omega t))$, (B.1) simplifies to:

$$
y(t)=\sum_{p=1}^{\infty} A_{p} \sin (p \cdot \omega(t)),
$$

where $A_{p}$ is the harmonic of the input signal with index $p$ and can be defined as a fourier series with coefficients described in terms of bessel functions $J_{p}[1,2]$ :

$$
A_{p}=\delta_{p 1} A+\sum_{n=1}^{\infty} \frac{2}{n \pi} J_{p}(2 n \pi A),
$$

where $\delta_{p 1}=1$ if $p=1$ and else $\delta_{p 1}=0$. Since the output consist of only odd harmonics, $A_{p}$ is zero for even values of $p$. 
By using (B.2), the harmonic distortion of a B-bit converter for a sine-wave input can be expressed as [3]:

$$
A_{3,1}=\frac{\sum_{n=1}^{\infty} \frac{2}{n \pi} J_{3}\left(2 n \pi A_{i n}\right)}{A_{i n}+\sum_{n=1}^{\infty} \frac{2}{n \pi} J_{1}\left(2 n \pi A_{i n}\right)}
$$

where $A_{\text {in }}=2^{B-1}$ is the maximum input amplitude. In the case of a two-tone input signal with an amplitude of $A_{i n, 1 \& 2}=A_{m} / 2$, the $3^{r d}$ order intermodulation product $\left(\mathrm{IM}_{3}\right)$ can be expressed as [3]:

$$
A_{21,1}=\frac{\sum_{n=1}^{\infty} \frac{2}{n \pi} J_{1}\left(n \pi A_{m}\right) J_{2}\left(n \pi A_{m}\right)}{0.5 A_{m}+\sum_{n=1}^{\infty} \frac{2}{n \pi} J_{1}\left(n \pi A_{m}\right) J_{0}\left(n \pi A_{m}\right)} .
$$

where $A_{21,1}$ represents the $\mathrm{IM}_{3}$ located at $2 f_{2}+f_{1}$.

\section{References}

[1] N. Blachman, "The intermodulation and distortion due to quantization of sinusoids," in Acoustics, Speech and Signal Processing, IEEE Transactions on, vol. 33, no. 6, pp. 1417-1426, Dec. 1985.

[2] J. v. Engelen and R. v. d. Plassche, Bandpass Sigma Delta Modulators Stability Analysis, Performance and Design Aspects. Springer, 2000.

[3] R. van de Plassche, CMOS Integrated Analog-to-Digital and Digital-toAnalog Converters. Dordrecht, the Netherlands: Kluwer Academic Publishers, 2003. 


\section{Summary}

This thesis describes the theory, design and implementation of a high-speed, high-performance continuous-time delta-sigma $(\mathrm{CT} \Delta \Sigma) \mathrm{ADC}$ for applications such as medical imaging, high-definition video processing, and wireline and wireless communications. In order to achieve a $\mathrm{GHz}$ clocking speed, this thesis investigates excess loop delay compensation techniques at the system level which enable the design of a wide-bandwidth (BW), high-dynamic range (DR) $\mathrm{CT} \Delta \Sigma$ modulator with good power-efficiency. The performance of a high-speed multi-bit $\mathrm{CT} \Delta \Sigma$ modulator is often limited by the dynamic errors present in the feedback DAC. The applicable correction/calibration techniques are limited due to the modulator stability requirements. We have implemented a dynamic error correction technique which not only experimentally quantifies the level of dynamic errors but also improves the dynamic performance of the modulator.

Chapter 2 discusses the system-level modeling of an ideal single-loop CT $\Delta \Sigma$ modulator and describes its major building blocks, i.e. the loop filter, quantizer, and DAC. The building-blocks of the modulator have been analyzed and their characteristic properties are presented to provide a basic understanding of the modulator. The stability of the delta-sigma modulator $(\Delta \Sigma \mathrm{M})$ is discussed and the relation between the stability and the building blocks is presented. To design a stable modulator with a high-order loop filter, the phase shift of the loop filter must be close to $90^{\circ}$ at $f_{s} / 2$. However, this condition is not sufficient to guarantee a stable modulator, as has been verified with computer simulations. Furthermore, Chapter 2 presents the effect of system-level non-idealities on the key performance metrics such as the signal-to-noise ratio (SNR), spurious-free dynamic range (SFDR), and $f_{s}$. The system-level non-idealities such as noise, linearity, metastability and excess loop delay (ELD) limit the performance of the modulator and their effect can be quantified by the system-level simula- 
tions. The loop filter and feedback DAC are the most dominant sources of noise which limit the dynamic range. Moreover, a multi-bit digital-to-analog converter (DAC) degrades the linearity due to the static and dynamic errors. The metastability of the quantizer, which is modeled as white noise added to the output of the quantizer, is not negligible at high sampling rates and degrades the SNR. However, if the modulator itself suffers from metastability, the signal-to-quantization noise ratio (SQNR) of the modulator degrades more dramatically. At a $\mathrm{GHz}$ sampling frequency, the ELD of the quantizer results in an unstable modulator. The ELD up to one clock period can be compensated with an extra feedback DAC around the quantizer without degrading the performance of the modulator.

Chapter 3 focuses on the design of $\mathrm{CT} \Delta \Sigma$ modulators at high-sampling rates. The system-level non-idealities discussed in Chapter 2 pose a major limitation at $\mathrm{GHz}$ sampling rates, and limit the possible architectural implementations. This chapter presents the system-level trade-offs in a single-loop $\Delta \Sigma \mathrm{M}$ and proposes a $3^{\text {rd }}$ order multi-bit $\Delta \Sigma \mathrm{M}$ which can achieve an $80 \mathrm{~dB}$ SQNR in a $125 \mathrm{MHz} \mathrm{BW}$ at a sampling rate of $4 \mathrm{GHz}$.

This architecture is found to be a good trade-off between circuit complexity and sampling speed in the target fabrication process which is 45-nm CMOS. To achieve a stable modulator sampled at $4 \mathrm{GHz}$, the latency of the multi-bit quantizer and the limited gain-bandwidth product (GBW) of the amplifiers, which degrade the SQNR, are modeled at the system level. To implement a power-efficient modulator, a high-speed capacitive-feedforward loop filter architecture has been proposed. The first two integrators are implemented as $\mathrm{RC}$ integrators since these can operate at low supply voltages while providing the required linearity of $-70 \mathrm{~dB}$ THD. The third integrator is a $g_{m} C$ integrator since requirements on its linearity are relaxed by the gain of the first two integrators. Using a $g_{m} C$ integrator as the last stage enables implementation of the summation node by employing a digital differentiated DAC whose output current is integrated on the output capacitance of the loop filter.

The system-level noise breakdown of the modulator defines the requirements of the building blocks of the modulator. The loop filter is optimized for the thermal noise, linearity, and finite GBW of its amplifiers. Moreover, a detailed analysis of the finite transconductance $\left(g_{m}\right)$ of the amplifier reveals that the non-linear input stage mixes down the high-frequency quantization noise into a signal band, and reducing the SQNR. The multi-bit quantizer defines the maximum sampling frequency. In order to reduce the latency, a flash quantizer architecture is preferred, and the architecture of the quantizer is defined to reduce the bit-error-ratio (BER) of the modulator. Furthermore, the offset voltage of the multi-bit quantizer limits the SFDR, and therefore must be 
sized to meet the linearity specification. However, this increases the device dimensions, which increases parasitics and therefore the ELD. For an inputreferred $\sigma_{V_{O S}}$ which is less than $V_{L S B} / 8$, the quantizer meets both the stability and linearity requirements.

The feedback DAC (DAC1) has the most stringent design requirements since it is directly connected to the input of the modulator. DAC1 is designed for 11bit matching, which is found to be a good compromise between sampling speed and low-frequency matching, the latter defining the area of DAC1. Moreover, $\mathrm{DAC} 1$ requires an output-current noise density of less than $0.01 \mathrm{nA} / \sqrt{\mathrm{Hz}}$, and clock jitter (RMS) smaller than 250fsec. DAC2 of the modulator, which is added to stabilize the modulator, has a much more relaxed design specification when compared to DAC1. Its non-idealities are shaped by the resonator of the loop filter. DAC2 requires a 9-bit matching to achieve a 70dB SNDR, and 1.5psec clock jitter (RMS) to achieve an $80 \mathrm{~dB}$ SNDR, which are derived from the system-level simulations.

Chapter 4 describes the implementation details of a $4 \mathrm{GHz} \mathrm{CT} \Delta \Sigma$ analog-todigital converter (ADC), which uses a high-speed capacitive-feedforward loop filter architecture, as proposed in Chapter 3. The ADC is implemented in 45nm-LP CMOS and achieves a 70dB DR and -74dBFS total harmonic distortion (THD) in a $125 \mathrm{MHz}$ BW. The system-level optimization and design tradeoff led to a state-of-the-art design in nm-CMOS where the low-supply voltage poses stringent noise requirements for the DR. The quantizer and the feedback DACs are co-designed to meet the latency and noise requirements. DAC1 is designed as resistively degenerated PMOS current sources with a supply voltage of $1.8 \mathrm{~V}$ to meet the matching and noise requirements. The low-frequency linearity of DAC1 is limited by the device matching, and the current sources are scaled for 11-bit matching. Since clock buffers consume considerable dynamic power, each quantizer slice consists of three stages: a preamplifier, a latch and a D-FF. The latency of the quantizer must be less than half a sampling-clock period (125ps) to ensure the stability of the modulator. Its kickback on the loop filter and reference ladder is minimized by biasing the preamplifier and the latch of the quantizer with static current. The kickback of the D-FF is attenuated by the gain of the first two stages of the quantizer. The clocking scheme of the quantizer and the feedback DACs are important for the stability of the modulator, therefore this chapter presents the detailed timing diagram of the modulator. The input stage uses a two-stage feedforward-compensated amplifier which achieves an 8GHz unity-gain-bandwidth (UGBW). The implemented ADC is characterized by using a custom designed measurement setup. The detailed measurement results are presented with particular focus on the jitter performance of the ADC. 
In order to improve the SNR and signal-to-noise-and-distortion ratio (SNDR) of the modulator, the non-linearity of the multi-bit DAC1 must be corrected or calibrated without degrading the stability, and power efficiency of the modulator. The static errors of DAC1 can be calibrated to improve the low-frequency linearity of the modulator. However, at a $\mathrm{GHz}$ sampling rate, the dynamic errors of DAC1 become more dominant, which makes correcting the dynamic errors without degrading the modulator stability a major challenge. Chapter 5 describes a multi-mode implementation of the multi-bit CT $\Delta \Sigma$ ADC, which is sampled at $2 \mathrm{GHz}$. The dynamic errors of its multi-bit DAC are eliminated by using an error switching (ES) scheme at the virtual ground node of the first integrator. This technique prevents the loop filter from processing the dynamic errors of the feedback DAC and improves the SNR, SNDR, and THD performance approximately by $3 \mathrm{~dB}$, resulting in a $25 \%$ improvement in the power efficiency of the modulator.

This thesis describes, in nanometer CMOS, continuous-time delta-sigma $(\mathrm{CT} \Delta \Sigma)$ ADCs are a power efficient alternative to Nyquist-rate ADCs for wide signal bandwidths (greater than $100 \mathrm{MHz}$ ) and high dynamic ranges (more than 12-bit). 


\section{Samenvatting}

Dit proefschrift beschrijft de theorie, het ontwerp en de implementatie van een zeer snelle, high-performance tijdscontinue delta-sigma (CT $\Delta \Sigma$ ) ADC voor applicaties zoals medische beeldvorming, HD video bewerking, communicatie via kabel en draadloze communicatie. Om kloksnelheden in het $\mathrm{GHz}$ bereik te realiseren onderzoekt dit proefschrift compensatie technieken voor vertragingen in de lus op systeem niveau, hetgeen het ontwerp van een breedband, hoge resolutie CT $\Delta \Sigma$ modulator met een goede vermogensefficiëntie mogelijk maakt. De prestatie van een zeer snelle, multi-bit $\mathrm{CT} \Delta \Sigma$ wordt vaak gelimiteerd door dynamische fouten in de terugkoppel DAC. De correctie/calibratie technieken die kunnen worden toegepast zijn beperkt vanwege de stabiliteitseisen. We hebben een dynamische fouten correctie techniek geïmplementeerd die niet alleen experimenteel het niveau van de dynamische fouten kwantificeert, maar ook de dynamische prestatie van de modulator verbetert.

Hoofdstuk 2 behandelt de modellering op systeem niveau van een ideale enkele lus CT $\Delta \Sigma$ modulator en beschrijft de meest belangrijke blokken, zoals het lus filter, quantisator en DAC. De bouwblokken van de modulator worden geanalyseerd en hun karakteristieke eigenschappen worden gepresenteerd om basis begrip van de modulator op te bouwen. De stabiliteit van de delta-sigma modulator $(\Delta \Sigma \mathrm{M})$ wordt besproken en de relatie tussen de stabiliteit en de bouwblokken wordt gepresenteerd. Om een stabiele modulator te ontwerpen met een hoge orde lus filter moet de fase verschuiving van het lus filter vlak bij de 90 graden zijn op fs/2. Deze conditie is echter niet genoeg om een stabiele modulator te garanderen, zoals is geverifieerd met computer simulaties. Verder wordt in hoofdstuk 2 het effect van niet idealiteiten op systeem niveau op belangrijke prestatie getallen zoals de signaal-ruis verhouding (SNR), spurious-free dynamisch bereik (SFDR) en klok frequentie fs. De systeem niet- 
idealiteiten zoals ruis, vervorming, metastabiliteit en lus vertraging (ELD) beperken de prestatie van de modulator en hun effect kan worden gekwantificeerd door systeem simulaties. Het lus filter en de terugkoppel DAC zijn de meeste dominante bronnen van ruis die het dynamisch bereik beperken. Daarbovenop degradeert een multi-bit DAC de lineariteit vanwege statische en dynamische fouten. De metastabiliteit van de quantizer, die wordt gemodelleerd als witte ruis aan de uitgang van de quantizer, kan niet worden verwaarloosd op hoge frequenties en verslechtert de SNR. Echter, vanwege het feit dat de modulator zelf last kan hebben van metastabiliteit, verslechtert de signaal-kwantisatie ruis verhouding (SQNR) nog dramatischer. Bij een $\mathrm{GHz}$ bemonsteringsfrequentie van een zorgt de ELD van de quantizer voor een instabiel systeem. De ELD kan tot 1 klok periode worden gecompenseerd met een extra terugkoppel DAC rond de quantizer zonder dat de prestatie van het systeem verslechtert.

Hoofdstuk 3 concentreert zich op het ontwerp van $\mathrm{CT} \Delta \Sigma$ modulatoren op hoge bemonsteringsfrequenties. De systeem niveau niet-idealiteiten die zijn besproken in hoofdstuk 2, vormen een belangrijke beperking op $\mathrm{GHz}$ bemonsteringsfrequenties en limiteren de mogelijke architectuur keuzes. Dit hoofdstuk presenteert de afwegingen op systeem niveau voor een enkele lus $\Delta \Sigma \mathrm{M}$ en een $3^{e}$ orde multi-bit $\Delta \Sigma \mathrm{M}$, die $80 \mathrm{~dB}$ SQNR in een $125 \mathrm{MHz}$ bandbreedte kan realiseren met een bemonsteringsfrequentie van $4 \mathrm{GHz}$.

Deze architectuur blijkt een goede afweging tussen circuit complexiteit en snelheid te zijn in de gekozen 45-nm CMOS fabricage technologie. Om een stabiele modulator op $4 \mathrm{GHz}$ te realiseren is de vertraging van de multi-bit quantizer en het beperkte versterking-bandbreedte product (GBW) van de versterkers, die de SQNR degraderen, gemodelleerd op systeem niveau. Om een vermogensefficiënte modulator te implementeren is een zeer snelle, capacitiefvoorwaarts gekoppeld, lus filter architectuur voorgesteld. De eerste 2 integratoren zijn geïmplementeerd als RC integratoren omdat deze op een lage voedingsspanning kunnen werken terwijl ze -70dB THD lineariteit kunnen halen. De derde integrator is een gmC integrator omdat de lineariteitseisen van deze integrator worden versoepeld door de versterking van de eerste 2 integratoren. Het gebruik van een $g_{m} C$ integrator als laatste trap maakt het mogelijk om het optelpunt te implementeren middels een gedifferentieerde DAC waarvan de uitgangsstroom wordt geïntegreerd in de uitgangscapaciteit van het lus filter.

De ruisverdeling op systeem niveau bepaalt de eisen voor de bouwblokken van de modulator. Het lus filter is geoptimaliseerd voor thermische ruis, lineariteit en eindige GBW van de versterkers. Bovendien laat een gedetailleerde analyse van de eindige transconductantie $\left(g_{m}\right)$ van de versterker zien dat de niet lineaire ingangstrap hoog frequent kwantisatie ruis in de signaal band mengt en de SQNR reduceert. De multi-bit quantizer bepaalt de maxi- 
male bemonsteringsfrequentie. Om de vertraging te reduceren heeft een flash architectuur de voorkeur. De comparator architectuur is bepaald om de biterror-rate (BER) van de modulator te reduceren. De offset spanning van de multi-bit quantizer limiteert de SFDR en moet zodanig geschaald worden om de lineariteit specificatie te halen. Echter, dit zorgt ervoor dat de afmetingen van de transistoren toeneemt en daarmee de vertraging. Voor een naar de ingang gerelateerde offset $\sigma_{V_{O S}}$ die kleiner is dan $V_{L S B} / 8$ voldoet de quantizer zowel aan de stabiliteitseisen als aan de lineariteitseisen.

De terugkoppel DAC (DAC1) heeft de meest strenge ontwerpeisen omdat die direct aan de ingang van de modulator is gekoppeld. DAC1 is ontworpen voor 11-bit matching, wat een goed compromis is tussen snelheid en matching op lage frequenties, dat het oppervlak van DAC1 bepaalt. Bovendien moet de ruisdichtheid van de uitgangsstroom van DAC1 minder dan $0.01 n A / \sqrt{H z}$ zijn en de klok jitter (RMS) minder dan 250fsec. DAC2 van de modulator is toegevoegd om de modulator te stabiliseren en heeft veel minder strenge ontwerp eisen dan DAC1. Zijn niet-idealiteiten worden onderdrukt door de resonator van het lus filter. Systeem simulaties laten zien dat DAC2 9-bit matching nodig heeft om 70dB SNDR te bereiken en 1.5psec klok jitter (RMS) om 80dB SNDR te behalen.

Hoofdstuk 4 beschrijft de implementatie details van een $4 \mathrm{GHz} \mathrm{CT} \Delta \Sigma \mathrm{ADC}$ met een zeer snelle capacitieve voorwaarts gekoppelde architectuur, zoals voorgesteld in hoofdstuk 3. De ADC is geïmplementeerd in een 45nm-LP CMOS technologie en behaalt 70dB DR en $-74 \mathrm{dBFS}$ harmonische vervorming (THD) in $125 \mathrm{MHz}$ bandbreedte. Systeem niveau optimalisatie en ontwerp afwegingen hebben geleid tot een state-of-the-art ontwerp in nm-CMOS waar de lage voedingsspanning strenge ruis eisen stelt voor het DR. De quantizer en de feedback DAC zijn als 1 geheel ontworpen om de vertraging en ruis eisen te halen. DAC1 is ontworpen met weerstand-gedegenereerde PMOS stroombronnen met een voedingsspanning van $1.8 \mathrm{~V}$ om de matching en ruis eisen te halen. De lineariteit van DAC1 op lage frequenties wordt beperkt door de matching tussen de componenten en de stroombronnen, en zijn ontworpen voor 11-bit matching. Omdat de klok buffers een aanzienlijk vermogen verbruiken bestaat elke quantizer eenheid uit 3 trappen; een voorversterker, een latch en een D flip-flop (D-FF). De vertraging van de quantizer moet minder dan een halve klok periode (125psec) zijn om de stabiliteit van de modulator te garanderen. De kickback op het lus filter en de referentie ladder is geminimaliseerd door de voorversterker en de latch met een statische stroom te voeden. De kickback van de D-FF wordt verzwakt door de versterking van de eerste 2 trappen van de quantizer. Het klokschema van de quantizer en de terugkoppel DACs zijn belangrijk voor de stabiliteit van de modulator. De ingangstrap bestaat 
uit een 2-traps voorwaarts gecompenseerde versterker met een $8 \mathrm{GHz}$ eenheidsversterking-bandbreedte (UGBW). De geïmplementeerde ADC is gemeten met een speciaal ontworpen meetopstelling. De gedetailleerde meetresultaten worden gepresenteerd met een specifieke focus op de jitter gevoeligheid van de ADC.

Om de SNR en SNDR van de modulator te verbeteren moet de nietlineariteit van multi-bit DAC1 gecorrigeerd of gekalibreerd worden zonder de stabiliteit en vermogensefficiëntie te verslechteren. De statische fouten van DAC1 kunnen worden gekalibreerd om de laag frequente lineariteit van de modulator te verbeteren. De dynamische fouten van DAC1 worden echter meer dominant in het geval van een $\mathrm{GHz}$ bemonsteringsfrequentie, wat het corrigeren ervan zonder de stabiliteit aan te tasten tot een zeer grote uitdaging maakt. Hoofdstuk 5 beschrijft een multi-mode implementatie van de multi-bit CT $\Delta \Sigma$ ADC die op $2 \mathrm{GHz}$ wordt geklokt. De dynamische fouten van de multi-bit DAC worden geëlimineerd met behulp van een fouten schakelschema (ES) op het virtueel aardpunt van de eerste integrator. Deze techniek voorkomt dat het lus filter dynamische fouten van de terugkoppel DAC te verwerken krijgt en verbetert de SNR, SNDR en THD prestatie met ongeveer $3 \mathrm{~dB}$ wat een verbetering in de vermogensefficiëntie bewerkstelligt van $25 \%$.

Dit proefschrift, voor de conversie van grote signaal bandbreedtes (groter dan $100 \mathrm{MHz}$ ) en hoog dynamisch bereik (meer dan 12-bits), zijn tijdscontinue delta-sigma ADCs een vermogensefficiënt alternatief voor Nyquist-rate ADCs als de ADC in een nanometer technologie geïmplementeerd moet worden. 


\section{List of publications}

\section{Journal papers}

- M. Bolatkale, L. Breems, R. Rutten, and K. Makinwa, "A 4 GHz ContinuousTime $\Delta \Sigma$ ADC With $70 \mathrm{~dB}$ DR and $74 \mathrm{dBFS}$ THD in $125 \mathrm{MHz} \mathrm{BW}$," Solid-State Circuits, IEEE Journal of, vol. 46, no. 12, pp. $2857-2868$, Dec. 2011.

- M. Bolatkale, M. Pertijs, W. Kindt, J. Huijsing, and K. Makinwa, "A Single-Temperature Trimming Technique for MOS-Input Operational Amplifiers Achieving $0.33 \mathrm{~V} /{ }^{\circ} \mathrm{C}$ Offset Drift," Solid-State Circuits, IEEE Journal of, vol. 46, no. 9, pp. 2099 -2107, Sept. 2011.

\section{Conference papers}

- M. Bolatkale, L. Breems, R. Rutten, and K. Makinwa, "A 4GHz CT $\Delta \Sigma$ ADC with 70dB DR and -74dBFS THD in 125MHz BW," in Solid-State Circuits Conference Digest of Technical Papers (ISSCC), 2011 IEEE International, Feb. 2011, pp. $470-472$.

- M. Bolatkale, M. Pertijs, W. Kindt, J. Huijsing, and K. Makinwa, "A BiCMOS Operational Amplifier Achieving $0.33 \mathrm{~V} /{ }^{\circ} \mathrm{C}$ Offset Drift using Room-Temperature Trimming," in Solid-State Circuits Conference, 2008. ISSCC 2008. Digest of Technical Papers. IEEE International, Feb. 2008, pp. $76-596$. 


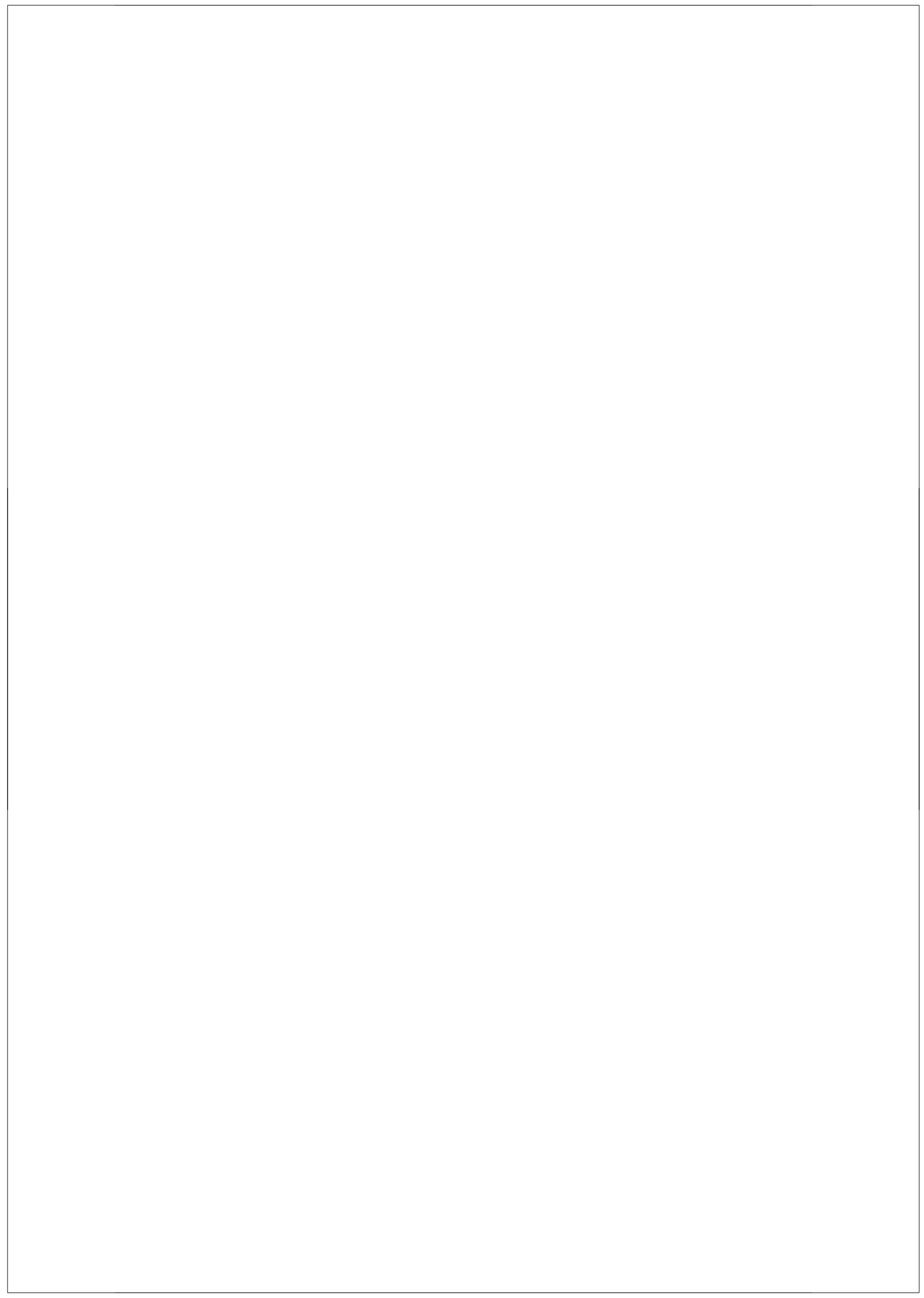




\section{About the author}

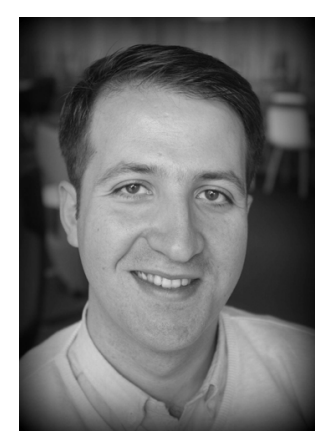

Muhammed Bolatkale received the B.S. degree in electrical engineering (high honors) from Middle East Technical University, Turkey, in 2004, and the M.Sc. degree in microelectronics (cum laude) from Delft University of Technology in 2007.

In 2007, he joined NXP Semiconductors, The Netherlands, where he carried his PhD research on high-speed Sigma-Delta ADCs in collaboration with Delft University of Technology. His main research interests include data converters, mixed-signal circuit design, and precision interface electronics. He received the ISSCC 2011 Jan Van Vessem Award for Outstanding European Paper and the IEEE Journal of Solid-State Circuits 2011 Best Paper Award for the paper entitled "A $4 \mathrm{GHz} \mathrm{CT} \Delta \Sigma \mathrm{ADC}$ with $70 \mathrm{~dB}$ DR and $-74 \mathrm{dBFS}$ THD in $125 \mathrm{MHz}$ BW". 


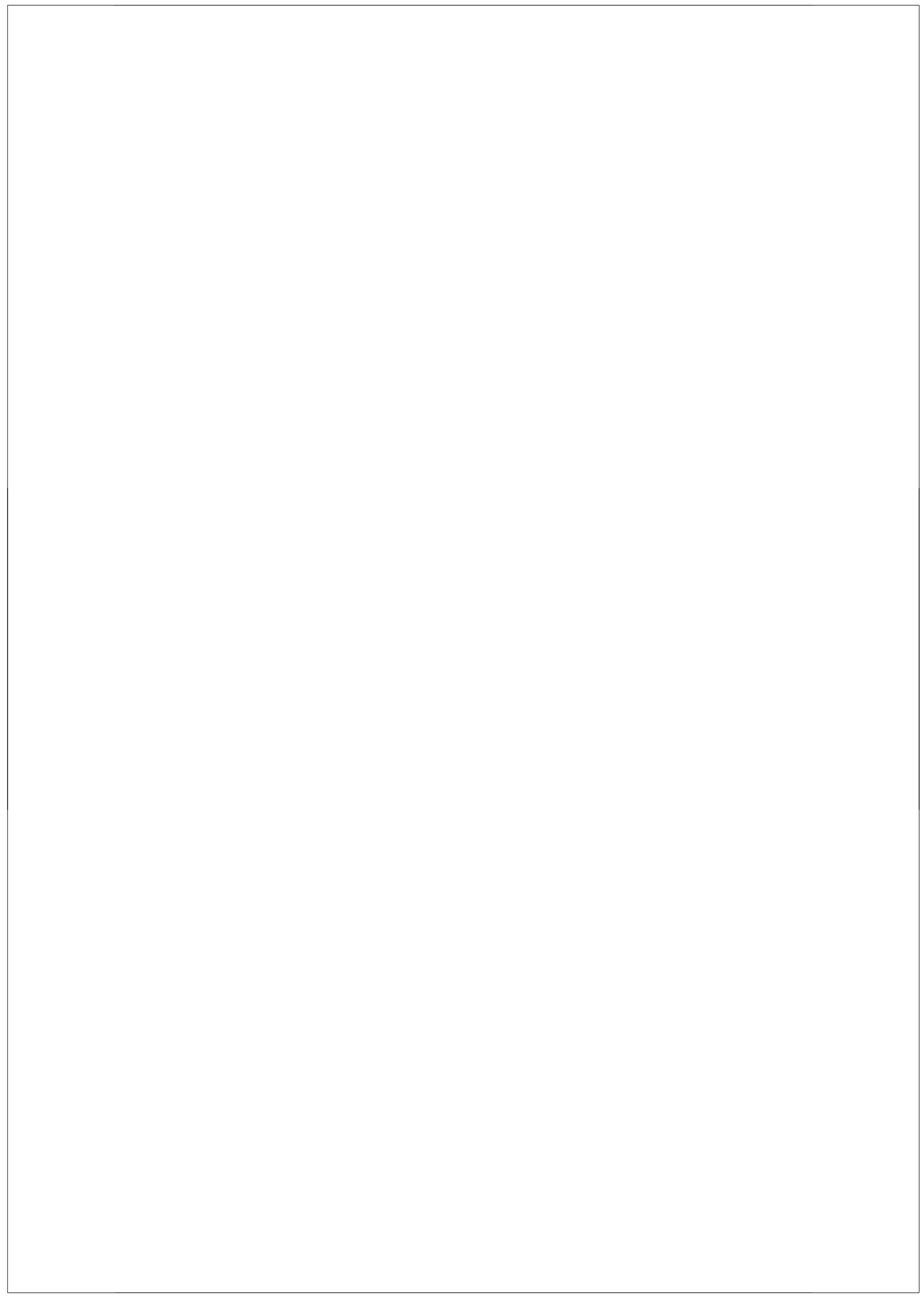




\section{Acknowledgments}

$\mathrm{A} \mathrm{PhD} \mathrm{is} \mathrm{more} \mathrm{than} \mathrm{a} \mathrm{journey} \mathrm{and} \mathrm{its} \mathrm{aim} \mathrm{is} \mathrm{not} \mathrm{only} \mathrm{to} \mathrm{focus} \mathrm{on} \mathrm{a} \mathrm{topic} \mathrm{in} \mathrm{depth}$ and contribute to academia but also to enjoy the journey. I have appreciated the value of intellectual diversity during my $\mathrm{PhD}$ thanks to the framework of this thesis. This work has been fully funded by NXP Semiconductors and I had the privilege and freedom to do my $\mathrm{PhD}$ as a member of the research department. It has been very interesting and educating to carry out $\mathrm{PhD}$ research and experience the dynamics of a semiconductor company.

First of all, I would like to thank my first promotor, Kofi Makinwa, who believed in me and helped me to draw a bigger picture during my $\mathrm{PhD}$ research quest. I have enjoyed his critical thinking and questions about my work, but soon I understand that to ask the right question you need to listen actively. He introduced me to the Delft style, where we always have a research objective to achieve the best in-class or to explore the unknown. His drive for quality will always inspire me as a person. His attitude for his students is more than what you could expect from an advisor. Thanks for your sincere care about my progress.

My second promotor, Lucien Breems, who not only introduced me to the field of delta-sigma analog-to-digital converters, but also helped me to understand the stability of delta-sigma modulators. This thesis would not have been possible without his support. During the most difficult part of this work, when you are behind the measurement bench and wondering how to debug the test chip, he shared very valuable insights that help me to experience the fun of measuring high-accuracy ADCs. Moreover, I would always appreciate your drive for focus and positive attitude about research. I also want to thank you for translating the summary of this thesis and the propositions into Dutch.

During the first three years of my PhD thesis, I had the privilege to have 
Leo Warmerdam as my manager, who offered me the possibility to be a member of NXP Research and initiated this project. He always motivated me to focus on the industrial aim of my $\mathrm{PhD}$ project. As my $\mathrm{PhD}$ work progressed, I appreciated his interest and valuable suggestions.

I would like to thank especially Kostas Doris, who is my current manager in the high-speed data-acquisition group. He facilitated the perfect environment in which to finalize my $\mathrm{PhD}$ work. Moreover, his expertise on high-speed digital-to-analog converters was very valuable for the outcome of my $\mathrm{PhD}$.

Special thanks go to my first roommates, Hans van de Vel and Berry Butter, with whom I shared my office during the first year of my $\mathrm{PhD}$ work. At that time, they were designing a state-of-the-art Nyquist ADC which not only broadened my understanding of ADCs but also shaped my research direction. Actually, the discussions that we had during the coffee breaks and their sincere interest in my work led to the specifications of our first prototype which turned out to be a very good fit for many industrial applications.

I would like to thank Robert Rutten whose digital design expertise and early involvement with the project was very important for the success of this thesis. Moreover, the expertise of Robert van Veldhoven on delta-sigma modulators and some of his early work on the high-speed design encouraged my progress. We had many discussions about the architectures-thank you for answering my questions and helping with the translations. Gerard van der Weide helped me a lot with the top-level challenges of microchips. His experience always showed me that we often underestimate the work required for a tape-out. I guess, we will always keep our optimism. Salvo Drago, who shared his knowledge on highspeed design and testing, was very valuable for my work. I want to especially thank Shagun Bajoria. We discussed through some of the most interesting aspects of delta-sigma modulators and his interest in the topic enabled very lively discussions. Thanks for reading my thesis and helping me to improve its quality.

Marcel Pelgrom and Maarten Vertregt have always inspired me with their experience and expertise. Marcel, thanks for sharing your wisdom on analogto-digital converter design and for your enthusiasm. Maarteen helped me a lot with understanding of ITRS roadmaps.

Fabio Sebastiano and I had the chance to experience the challenges of doing a $\mathrm{PhD}$ and having the same promotor. We had many discussion about technical and non-technical topics. Fabio introduced me to the Italian way of thinking and his beautiful country. I had great time there, and thanks again for introducing me to your family. You are a great friend.

While doing my $\mathrm{PhD}$, I had the privilege to spend time and discuss on many topics with colleagues from NXP Research. They deserve special thanks. First 
of all, the members of high-speed data-acquisition group Gerrit den Besten, Marcello Ganzerli, Erwin Janssen, Yu Lin, Alberto Rodriguez Perez, Athon Zanikopoulos, Vladislav Dyachenko and Alessandro Murroni. Moreover, Amine Mounaim, Harish Kundur, Jingjing Hu, Frank Leong, Gerard Villar Piqué, Ravi Karadi, Juan Osorio, Nenad Pavlovic, Tarik Saric, Mark van Schie, Cicero Vaucher, Melina Apostolidou, Remco van de Beek, Raf Roovers, Selçuk Ersoy, Onur Kaya, Victor Zieren, Henkjan Bergveld, Arnoud van der Wel, Hamed Fatemi, Maurice Meijer, and Jose Pineda de Gyvez created such a nice environment at NXP and I enjoyed it a lot.

During my $\mathrm{PhD}$ as a member of the Electronic Instrumentation Laboratory of TU Delft, I met many great people and friends who I will appreciate throughout my career. I want to thank Paulo Silva, Mahdi Kashmiri, Qinwen Fan, Caspar van Vroonhoven, Kamran Souri, André Aita, Maureen Meekel, Rong Wu, Zili Yu, Sha Xia, Zhichao Tan and Youngcheol Chae for being a part of my experience at TU Delft. I also want to thank our secretary Joyce Siemers who always kept me informed even though I was a remote PhD student.

I would like to thank Sarah van Galambos for proof reading this thesis. Her comments was very valuable and helped me to keep my focus on the content of the thesis.

Throughout the years I lived in Eindhoven, I met many great people some of whom are very special to me. I would like to thank Anna Maria Kuras, Emanuela Bosco, Agnese Bargagli, Jeroen Weenink, Javier Bescos, Greg Corcoran, Perrine and Alexis Dufourcq, Leopoldo Luongo, Milda and Gianluca Monaci, Johan Lundberg, Tommaso Gritti, Jeanne America, Lourdes Mestre, Ruben Rajagopalan, Mustafa Acar, Isabel de la Fuente, Murtaza Bulut, Ercan Şengil, Baki Tezel, Uğur Keskin, Mustafa Özen, Ergin Sarıkaya, Uğur Bağcı, and Suat Tüter for sharing a part their life with me and being good friends. I also want to thank the Italian community of Eindhoven, the Andricciolas, the Marchettis, the De Majos, the Fazzis, and Alberto Manconi. I guess I would have never experience the warmth of Italy without you.

This $\mathrm{PhD}$ thesis would not be possible without the unconditional support and encouragement of my family, and my brothers Mustafa and Resul. I feel privileged to have you in my life.

Anne ve baba, bana vermiş olduğunuz maddi ve manevi destekten dolayı çok teşekkür ederim. Kardeşlerim Mustafa ve Resul iyi ki varsınız. İnşallah hep beraber daha nice güzel günlere.

Muhammed Bolatkale,

The Netherlands, 23 September 2013. 


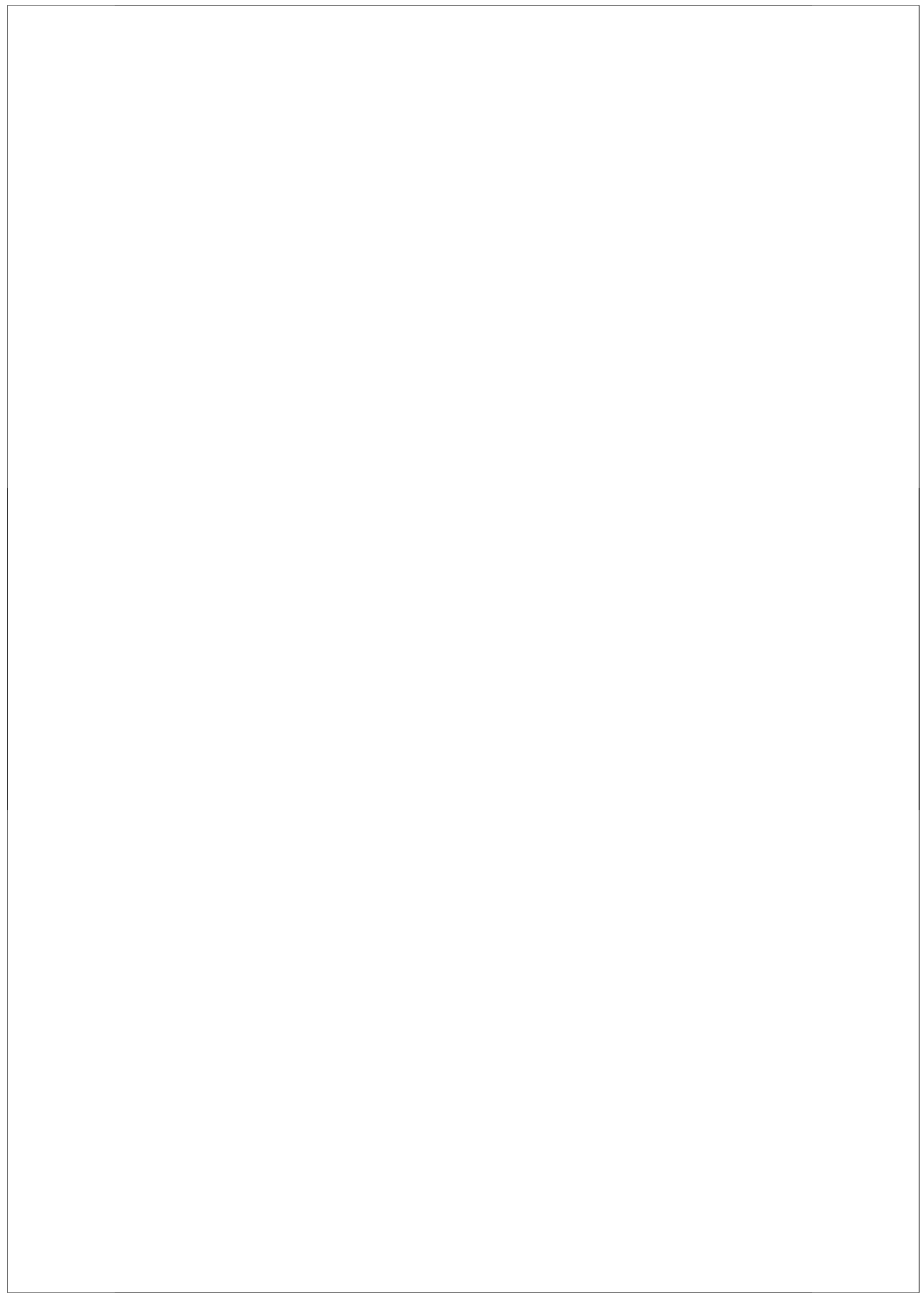

